\title{
THE EFFECT OF GENERAL IMPERFECTIONS \\ ON THE \\ BUCKLING OF CYLINDRICAL SHELLS
}

\author{
Thesis by \\ Johann Arbocz
}

In Partial Fulfillment of the Requirements

For the Degree of

Doctor of Philosophy

California Institute of Technology

Pasadena, California

1968

(Submitted May 1, 1968) 


\section{ACKNOWLEDGMENT}

The author wishes to express his sincere appreciation to Dr. E. E. Sechler for his guidance of the work carried out in this investigation; Dr. C. D. Babcock for his advice and comments; Messrs. Marvin Jessey, Clarence Hemphill and Richard Luntz for their help in setting up the experiments; Mrs. Betty Wood for her excellent drawings and Miss Helen Burrus for bearing with my handwriting and typing the manuscript so skillfully.

This study was supported in part by the National A.eronautics and Space Administration under Research Grant NsG-18-59 and this aid is gratefully acknowledged.

Finally the author is indebted to his wife and his daughter for their patience and encouragement during many days of graduate work. 


\section{ABST RACT}

An experimental and theoretical investigation of the effect of general imperfections on the buckling load of a circular cylindrical shell under axial compression was carried out.

A non-contact probe has been used to make complete imperfection surveys on electroformed copper shells before and during the loading process up to the buckling load. The data recording process has been fully automated and the data reduction was done on an IBM 7094. Three-dimensional plots were obtained of the measured initial imperfection surfaces and of the growth of these imperfections under increasing axial load. The modal components of the measured imperfection surfaces were also obtained.

The theoretical solution located the limit points of the postbuckled states. A simplified imperfection model was used consisting of one axisymmetric and one asymmetric component. For global buckling the correlation between the theoretical buckling loads and the experimental values was found to be good. 


$$
\text { iv }
$$

\section{TABLE OF CONTENTS}

PART

PAGE

I

INT RODUCTION

II EXPERIMENTAL PROGRAM

1. Test Equipment

a. Traversing Mechanism

b. Model Control Unit

c. Pick-up System

6

d. Data Control Unit 7

e. Digital Voltmeter 8

f. Card Punch 8

2. Check-out of the Test Equipment 8

a. Scans of Known Contours $\quad 8$

b. Circumferential Time Delay Check-out $\quad 10$

c. Hysteresis and Repeatability of the 11 Measured Data

3. Fabrication of the Test Specimen 12

a. Wall Thickness 12

b. Material Properties 13

4. Test Procedure 13

a. Load Cell 14

b. Calibration of the Pick-up 15

c. Installing the Test Shell 15

d. Initial Imperfection Measurements 16

e. Monitoring of the Strain Gages 17

f. Buckling Procedure 17 
TABLE OF CONTENTS (Cont'd)

5. Data Reduction - Main Program 18

a. Best Fit Polynomial to Pick-up 19

Calibration Data

b. Definition of the "Perfect" Cylinder 20

c. Finding Harmonic Components of the 21

Measured Imperfection Surface

6. Data Reduction - Auxiliary Programs 24

a. Three-Dimensional Plots 25

b. Computing the Growth of Harmonic 25

Components

7. Test Results 25

III COMPARISON WITH THEORY 30

1. Donnell's Shell Equations 31

2. Nonlinear Buckling Equations 32

3. Buckling of a Perfect Shell 38

4. Buckling of a Shell with Axisymmetric 39 Imperfection

5. Buckling of a Shell with Both Axisymmetric 41 and Asymmetric Imperfections

6. Numerical Results 45

IV CONCLUSION $\quad 49$ 
vi

TABLE OF CONTENTS (Cont'd)

PART

PAGE

REFERENCES

52

APPENDIX A

55

TABLES

64

FIGURES

152 


\section{vii \\ LIST OF TABLES}

TABLE

PAGE

I

Repeatability Checks

64

II Principal Components of the Initial Imperfection

66

Surface

III

Variation of the Best Fit "Perfect" Cylinder with

77

Load Increments

IV

Summary of Buckling Tests

85

V

Summary of "Critical" Fourier Coefficients

86

VI

Summary of Wavenumbers

110

VII

Numerical Results

111

VIII Comparison of Theory and Experiment 
viii

\section{LIST OF FIGURES}

FIGURE

PAGE

1 Traversing Mechanism

2 Partially Assembled Scanning Mechanism

3 Schematic Layout of the Shell Buckling Experiment

154

Measuring Imperfections

4 Model Control Unit

5

Pick-up Circuit

6 Micrometer Measurement of Known Contour

Compared to Pick-up Measurements

7 Constant Speed Traverse of Known Contour

Compared to "Static" Micrometer Measurement

Time Delay for Punch Control

Adjustment of Time Delay

10 Hysteresis Check

11 Testing Machine and Data Acquisition Equipment

12 Details of Testing Machine Loading Screw

14 Pick-up Calibration Set Up

15 Typical Pick-up Calibration Curve 165

16 Cylindrical Shell Testing Configuration 166

$17 \quad$ Layered Main Program 167

18 Best Fit Cylinder Reference Axis $\quad 168$

19 Initial Imperfection, Shell A7 $\quad 169$

20 Initial Imperfection, She11 A8 170 
ix

LIST OF FIGURES (Cont'd)

FIGURE

PAGE

21 Initial Imperfection, Shell A9

171

22 Initial Imperfection, Shell A10

172

23 Initial Imperfection, Shell A12

173

24 Prebuckling Deformation Growth at

174

$\mathrm{P} / \mathrm{P}_{\mathrm{cl}}=0.061$, Shell A7

25 Prebuckling Deformation Growth at

$\mathrm{P} / \mathrm{P}_{\mathrm{cl}}=0.325$, Shell A7

26 Prebuckling Deformation Growth at

176

$\mathrm{P}_{\mathrm{c}} \mathrm{P}_{\mathrm{c}}=0.539$, Shell A7

27 Local Buckling Deformation, Shell A7

177

28 Prebuckling Deformation Growth at

$\mathrm{P} / \mathrm{P}_{\mathrm{cl}}=0.637$, Shell A8

29 Prebuckling Deformation Growth at

$P / P_{c l}=0.722$, Shell A9

30 Prebuckling Deformation Growth at

$P / P_{c l}=0.439$, Shell Al0

31 Prebuckling Deformation Growth at

$\mathrm{P} / \mathrm{P}_{\mathrm{c} l}=0.594$, Shell Al2

Local Buckling Deformation, Shell A10

182

33 Post Buckling Deformation, Shell A7

183

34 Post Buckling Deformation, Shell A8

184

35 Post Buckling Deformation, Shell A9

36 Post Buckling Deformation, Shell A10 


\section{LIST OF FIGURES (Cont'd)}

FIGURE

PAGE

Load Distribution Near Buckling

39 Load Distribution Near Buckling

40 Growth of Fourier Coefficients for Shell A7

41 Growth of Fourier Coefficients for Shell A8

42. Growth of Fourier Coefficients for Shell A9

43 Growth of Fourier Coefficients for Shell Al0

193

44 Growth of Fourier Coefficients for Shell A12

194

45 Shell Geometry and Coordinate System

195

46 Post Buckling Equilibrium Paths for Perfect

196

Shells

47 Post Buckling Equilibrium Paths for Shells with Axisymmetric Imperfections

Post Buckling Equilibrium Paths for Shells with Axisymmetric and Asvmmetric Imperfections 


\section{LIST OF SYMBOLS}

$\lambda$$$
v
$$

$\mathrm{R}$

t

$\sigma$

E

$\delta$

V

$\mathrm{X}_{1}, \mathrm{Y}_{1}$

$\epsilon_{1}, \epsilon_{2}$

$\overline{\mathrm{w}}, \overline{\mathrm{W}}$

$x, y$

$\overline{\mathbf{x}}, \overline{\mathbf{y}}$

L

$A_{i j}$

${ }^{B}{ }_{i j}$

$\mathrm{C}_{\text {ij }}$

$D_{i j}$

Nondimensional loading parameter $\left(\lambda=\sqrt{3\left(1-\nu^{2}\right)} \frac{R}{t} \frac{\sigma}{E}\right)$

Poisson's Ratio

Shell radius

Shell thickness

Axial stress

Young's Modulus

Displacement of pick-up from conducting surface Pick-up output voltage

Coordinates locating origin of the best fit cylinder reference axis

Small angles in $x$ adians denoting the inclination of the best fit reference axis $\left(\epsilon_{1}=\pi / 2-\alpha ; \epsilon_{2}=\pi / 2-\beta\right.$ when using notation of Fig. 18) Radial imperfection from perfect circular cylinder Axial and circumferential coordinates on middle surface of shell, respectively

Nondimensional coordinates $\left(\bar{x}=\frac{2 \pi x}{L}, \bar{y}=\frac{y}{R}\right)$

Length of the shell

Coefficient of $\cos i \bar{y} \cdot \cos j \bar{x}$

Coefficient of $\sin i \bar{y} \cdot \cos j \bar{x}$

Coefficient of $\cos i \bar{y} \cdot \sin \mathrm{j} \bar{x}$

Coefficient of $\sin i \bar{y} \cdot \sin j \bar{x}$ 
xii

\section{LIST OF SYMBOLS (Cont'd)}

$\Delta$

$\bar{\xi}_{i}$

$\eta$

$\xi_{\mathbf{i}}$

$\mathrm{w}, \mathrm{W}$

$\mathbf{f}, \mathbf{F}$

i, $\mathbf{k}$

$\ell$

$\mathrm{K}_{1}, \mathrm{~K}_{2}, \ldots \mathrm{K}_{10}$

Nondimensional growth of initial imperfection after application of the ith load increment

Nondimensional initial imperfection amplitude

Nondimensional number indicating size of imperfection g rowth

Nondimensional radial displacement amplitude

Radial displacement

Airy stress function

Axial wave numbers

Circumferential wave number

Constants defined in Appendix A

A, B, ...J Constants defined in Appendix A

$\alpha, \alpha_{A}, \alpha_{1}, \alpha_{2}, \alpha_{3}, \beta \quad$ Mode shape parameters defined on page 35

$C_{1}, C_{2}, C_{3}, C_{4} \quad$ Constants defined on page 37 


\section{INTRODUCTION}

The stability of cylindrical shells under axial compression has been studied in the past both theoretically and experimentally by several investigators. Previously reported experimental studies (Ref. 1) showed that, when compared with the results predicted by the linearized small deflection theories of buckling, the experimental values were much lower and the data had a large scatter band. In the past decade the following five factors found acceptance as an explanation of the discrepancy between theory and experiments:

1. Initial geometrical imperfections of the order of a fraction of the wall thickness.

2. Nonuniformity of load distribution a round the shell circumference.

3. Influence of boundary conditions.

4. Effect of prebuckling deformation caused by edge constraints.

5. Nuclei of plastic strain.

The influence of the load distribution on the buckling load is unknown. Little is known about the effect of the nuclei of plastic strain. However the influence of the boundary conditions and the effect of prebuckling deformations caused by edge constraints has been extensively investigated (Refs. $2,3,4,5$, and 6$)$. Whereas for certain end conditions the solution of the linear buckling equations predicts buckling at $\lambda=0.5$, for the clamped boundary conditions which are encountered almost exclusively in experiments the solution predicts buckling at 
$\lambda=1.0$. Including the effect of prebuckling deformations due to the edge constraints will lower the buckling load to only about $\lambda=0.85$. Thus initial geometrical imperfections have come to be accepted as the main degrading factor in the load carrying capacity of cylindrical shells under axial compression. Since the pioneering paper by Donnell in 1934 (Ref. 7) there have been many refinements in the theoretical solutions (Refs. 8, 9, 10, 11, 12, and 13). Experimental studies, however, seem to have suffered under the very difficult task of accurately recording imperfections of the order of only fractions of the wall thicknesses.

The purpose of the work described in the main part of this thesis was to determine the effect of general initial imperfections on the buckling load of cylindrical shells under axial compression. This was done by carrying out complete imperfection surveys on the cylindrical shells before and during the loading process up to the buckling load. Also by tracing the growth of the initial imperfections during the loading process prior to buckling it was hoped to isolate those modal components which furnished the mechanism by which the initial imperfections reduced the buckling load. 


\section{EXPERIMENTAL PROGRAM}

The following sections contain a description of the instrumentation designed to carry out the imperfection measurements, an outline of the check-out procedure used to assure the proper functioning of the individual components of the experimental set-up and a brief discussion about the fabrication of the test specimens and the procedure used to carry out the buckling tests. An outline of the imperfection measurements and a discussion of the data reduction process is also included.

\section{Test Equipment}

a. Traversing Mechanism

The specifications of the experimental program called for a scanning device that would be adequate to pick-up and record imperfections of only fractions of the test specimen's wall thickness of $0.004 \mathrm{in.}$ In addition the scanning device had to travel both in the axial and in the circumferential directions in order to record a complete surface map of the shell being tested. The numbers $\mathrm{s}^{(1),(2)}$ etc. used in the following section refer to part numbers listed in Fig. 1.

The scanning device was built around an inductance-type, non-contacting pick-up which measured the air gap between the end of the pick-up and the conducting copper surface of the shell. The pickup was installed in a movable support ${ }^{(3)}$ which in turn was fixed to a long shaft ${ }^{(2)}$ protruding inside the shell being tested. The 22 in. long, 1-3/8 in. diameter stainless steel shaft was ground on a cylindrical grinder to a straightness of less than 0.0001 in. total indicator reading. 
This shaft was supported by two bronze bearings, which were pressed into an aluminum alloy supporting $\mathrm{ring}^{(1)}$ and then lapped to fit the shaft. For better adjustment, the bronze bearings were partially split and could be tightened or loosened by fine thread set screws. The shaft could be moved axially in and out by means of a leadscrew ${ }^{(5)}$ which was supported in a self-aligning bearing ${ }^{(66)}$ pressed into the end-piece ${ }^{(4)}$. This in turn, was supported by the outer shell ${ }^{(12)}$ consisting of a 5 in. diameter aluminum alloy tube. The leadscrew was driven by the axial motor ${ }^{(72)}$ installed on the rear plate ${ }^{(17)}$ of the endcap (16). The shaft was driven in the circumferential direction by the circumferential motor ${ }^{(71)}$ installed at the rear side of a supporting plate $^{(6)}$ which traveled back and forth with the shaft in the axial direction. Whenever the shaft was driven in the circumferential direction the axial drive had to be disengaged in order to avoid moving the shaft axially because of the advancing or retreating nut on the leadscrew. The disengagement was accomplished by de-energizing a magnetic coupling ${ }^{(70)}$. The circumferential position of the pick-up was determined by the print control consisting of a microswitch which was triggered by the switching disk ${ }^{(9)}$ which had 48 uniformly spaced notches machined into it. The traversing sequence which consisted of a circumferential scan followed by an axial advance was controlled by strategically spaced microswitches. It will be described in more detail in the section on the Model Control Unit. Figure 1 shows the as sembly drawing of the traversing mechanism, and the partially assembled scanning mechanism can be seen in Fig. 2 . 
A schematic layout of the shell buckling experiment measuring imperfections is shown in Fig. 3. The following sections contain a detailed description of the individual components of the overall system.

\section{b. Model Control Unit}

As mentioned earlier, during the imperfection measurements, the shaft of the traversing device supporting the noncontact probe was driven alternatingly in the axial and circumferential directions by small electric motors. The control of this automatic sequence was accomplished by a series of relays controlled by microswitches. Figure 4 shows a schematic layout of the model control unit. To start the automatic scanning sequence the shaft had to be in the home position. Only then did pressing of the start button energize relay ${ }^{(2)}$. Energizing the relay made power available to the circumferential motor which started rotating the shaft in the counterclockwise direction. At the same time, relay ${ }^{(6)}$ was energized, opening the clutch between the axial motor and the leadscrew to avoid a simultaneous axial advance. Upon completing its circumferential scan, the circular cam riding on the shaft tripped one of the circumferential limit switches. This stopped the circumferential motor, de-energized relay ${ }^{(6)}$ which engaged the clutch between the axial motor and the leadscrew. At the same time relay ${ }^{(2)}$ was also de-energized creating a pulse that operated relay ${ }^{(3)}$, that latched relay ${ }^{(4)}$, making power available to the axial motor which started advancing the shaft of the scanning device. Upon completing the preset axial advance the incremental switch was tripped stopping the axial motor and de-activating 
relay ${ }^{(4)}$. This resulted in a pulse on relay ${ }^{(1)}$, which latched relay ${ }^{(2)}$ starting the circumferential motor in the clockwise direction, and the whole sequence was repeated. The automatic scanning with intermittent circumferential scans and axial advances continued until the shaft completed its full axial advance tripping the upper axial limit switch which stopped the sequence. The circumferential and the axial position of the noncontact probe was monitored by the output of two helipots. One helipot was driven by the circumferential motion, the other by the axial motion of the shaft. The output of the circumferential position indicator helipot was also used to drive the $\mathrm{x}$-axis of the $\mathrm{xy}$-plotter which recorded the pick-up signal directly on the $y$-axis of the graph paper.

The control of the traversing device could also be switched from automatic to manual. Then the circumferential and axial motion were controlled by separate 3 -position switches eliminating the automatic sequencing. The completion of a full circumferential scan, or the full travel in the axial direction was determined by the limit switches.

c. Pick-up System

The pick-up circuit shown on Fig. 5 consisted of two inductance-type pick-ups, an oscillator, a differential amplifier and a demodulator. The pick-ups consisted of a coil with a powdered iron core excited by a $100 \mathrm{KHz}$ signal generated by the oscillator. The impedance of the coils to this signal changed as their electromagnetic fields were disturbed by the eddy currents generated in the external 
conducting surfaces. The reference pick-up was preset during the calibration of the active pick-up and its setting remained the same for any given test. By determining the change in impedance of the coil of the active pick-up the position of the external shell surface could be measured quite accurately. This was done by first amplifying the voltage across the active pick-up's coil 100 times on the differential amplifier, then demodulating this AC signal. The output of the demodulator, consisting of a DC voltage was then read on the digital voltmeter and recorded on cards by an IBM $526 \mathrm{card}$ punch. Using the reference pick-up and the differential amplifier increased the sensitivity of the system to 1.0 volt per 0.001 in. as compared to a sensitivity of 0.25 volt per 0.001 in. if the active pick-up was used alone.

\section{d. Data Control Unit}

As can be seen from Fig. 3, the same digital voltmeter was used to read the strain gages on the load cell and the demodulated DC signal of the pick-up used to measure the imperfections of the test shell. To control the sequence of the signals fed to the digital voltmeter, a data control unit was built which included a 26 channel stepping switch circuit. Twenty-four channels were connected to the strain gages on the load cell, the 25 th channel indicated the voltage of the power source used to energize the strain gage circuits, the 26 th channel was the home position. It read the demodulated DC signal of the imperfection measuring pick-up. Using the stepping switch made it possible to read the strain gages automatically at a preset rate. 
Channel 26 of the data control unit also included an adjustable time delay circuit. This circuit became necessary because of the finite sampling time required by the Cimron digital voltmeter. Thus when using channel 26 the punch signal from the circular cam trapped the output of the pick-up at that particular instant in the time delay circuit. This would allow time for the digital voltmeter to sample this signal and to come to an essentially constant reading. At the end of the preset time delay the circuit activated the card punch recording the last reading of the digital voltmeter.

\section{e. Digital Voltmeter}

The digital voltmeter used for the tests was made by Cimron Division of Lear Siegler Inc., Trademark Cimron, Model P9400B with a DC preamplifier mode $6812 \mathrm{~B}$. Balance time for a full scale change in the 5 digits was 300 milliseconds.

\section{f. Card Punch}

Recording of the data was done on an IBM 526 card punch with a capability of 15 characters per second. The format used to record the data was $9 F 8.4$ with the last 8 columns used to record the run number and the card number. The run number was dialed in through a parameter board and the number of cards used was monitored on a card counter connected to the card punch.

2. Check-out of the Test Equipment

a. Scans of Known Contours

In checking out the pick-up system, a surface of known contour was scanned with the pick-up and its output was recorded by 
the DVM card punch system, giving the recorded data in terms of voltages. Later this data was converted into displacements on an IBM 7094 by means of the pick-up's calibration curve. The shape of the contour obtained by the pick-up measurements was then crossplotted with the shape of the contour as measured very accurately by micrometer on an optical comparator. Figure 6 shows a cross-plot of pick-up measurements vs. micrometer measurements. The agreement between the two readings was excellent except for the regions adjacent to the sharp step where the pick-up measurements deviated from the exact shape due to the integ rating feature of the pick-up. The width of this region was approximately equal to the diameter of the pick-up.

Since the scanning of the shells with the pick-up was to be done continuously it was necessary to verify that the output of the digital voltmeter, which was recorded in set intervals automatically on cards, represented the true shape of the measured shell surface. The verification was done by scanning a carefully machined surface of known contour with the pick-up moving at constant speed and recording the output of the pick-up by the DVM card punch system. A sample of these data is shown in Fig. 7. The agreement between the pick-up measurements and the measurements made with a micrometer was once again very good except fot the regions close to sharp corners where the integrating property of the pick-up smoothed out the shape of the scanned surface. 


\section{b. Circumferential Time Delay Check-Out}

During the circumferential scans a circular cam rotating with the shaft triggered a microswitch at every $7.5^{\circ}$ (See Fig. 1 for details). This was the signal to the Model Control Unit to record the readings of the Digital Voltmeter on cards by the IBM card punch. The intermittent counterclockwise and clockwise circumferential motion resulted initially in misalignments of identical circumferential stations measured at different axial positions. This was caused by the fact that the circular cam triggered the microswitch at different positions depending upon whether it was moving clockwise or counterclockwise. This problem was solved by placing an adjustable time delay into the punch control circuit. Figure 8 explains in detail the triggering sequence with and without the time delay.

Proper adjustment of the time delay circuit was achieved with the help of an xy-plotter. The $\mathbf{x}$-axis was driven by the circumferential position indicator helipot, whereas the y-axis was connected across the punch relay. Thus every punch was registered as a square wave on the graph paper. Using the graphs of the counterclockwise and clockwise scans done side by side the deviations from the planned exact punch locations were read off without any difficulty. Figure 9 shows a picture of the counterclockwise and clockwise punch signals. With the help of these graphs it was possible to compute the deviations of the actual punch signals from the planned exact punch locations. It was found that the punch intervals were kept as $7.5^{\circ} \pm 0.5^{\circ}$. 
c. Hysteresis and Repeatability of the Measured Data

For the checkout of the system as a whole a thick-walled aluminum cylinder was used. This cylinder was machined out of standard thick-walled aluminum tubing and was precision honed so that its deviations from roundness and its deviations from the mean diameter combined were less than 0.0005 in. total indicator reading.

Basically it should not have mattered whether the circumferential scanning at any one axial station was done clockwise or counterclockwise. However due to friction in the bronze bearings and play in the overall mechanical system it was expected that a certain amount of hysteresis would exist between the counterclockwise and clockwise scans at any one axial station. Figure 10 shows plots of the pick-up output during counterclockwise and clockwise circumferential scans at three different axial stations. The maximum deviation at any one point between the clockwise and counterclockwise scans was less than 0.0003 in., for most part of the plot less than 0.0001 in. It should be remarked here that originally, before making the bronze bearings adjustable the hysteresis between the clockwise and counterclockwise outputs was of the order of $0.001 \mathrm{in}$.

The final checkout of the system as a whole consisted of the complete surface scan of the near perfect thick-walled aluminum cylinder. The data were punched on cards, ran through the data reduction program and it was found that the maximum amplitude of any one of the computed harmonics was less than 0.0006 in. This was in 
good agreement with the previously mentioned tolerances of fabrication of this cylinder.

This complete surface scan.was repeated at a later date and the reduced data compared with the results of the first scan. Repeatability was found to be very good. Table I gives some data on these measurements.

\section{Fabrication of the Test Specimen}

The cylindrical shells used for this testing program were fabricated by electroforming on wax mandrels. This process was used previously in other investigations (Ref. 14). About an inch thick layer of wax was first cast on water cooled mandrels. After the wax had hardened it was machined to the desired dimensions on a lathe and spray painted with a silver paint thinned with Toluene. The plating was carried out in a Copper Fluoborate bath. Because of previous experience, copper was retained as the plating material. The plating time was about 20 minutes per 0.001 in. of plate. After the plating was completed, the mandrel was rinsed thoroughly and the shell cut to the desired length while it was still on the mandrel. After the cutting operation the shell was then removed from the mandrel by melting out the wax. The excess wax and silver paint was removed from the finished shells with benzene. For a more detailed description of the plating process see references 14 and 15 .

a. Wall Thickness

The average thickness of the test shells was determined before the buckling tests by weighing the shells. The specific gravity 
used in the calculation of the thickness was 8.9. The thickness variation along the generator of the shells was determined by cutting strips out of the first test specimens and determining their thickness by a comparator. The thickness variation was found to be less than \pm 2 per cent of the nominal value (See also Ref. 14).

\section{b. Material Properties}

Tests to determine the material properties of the plated copper were carried out in uniaxial tension. This was done by utilizing long strips of the plated copper which were soldered into $1 / 8$ in. thick plates that were in turn clamped into the jaws of an Instron testing machine. The strips had length to width ratios of about 80 . The head displacement of the testing machine was used as the measure of strain and the load read from the Instron load cell.

A determination of Poisson's ratio for each shell was not attempted since its influence in the reduction of the buckling data is of secondary importance. A value of 0.3 was used for this purpose.

\section{Test Procedure}

The buckling tests were carried out in the controlled enddisplacement type testing machine shown in Fig. 11. By the use of matched pairs of high precision thrust bearings the axial elastic displacement under load of the testing machine was kept to a minimum thus making the testing machine rigid in comparison with the test specimen. The relative displacement of the two end plates of the testing machine was controlled by four loading screws, which could be adjusted independently to give the proper load distribution on the 
shell, or turned simultaneously to increase the load up to the critical value. One complete turn of the screws gave a displacement of 0.025 in. Figure 12 shows the details of the se displacement controlling screws. The springs shown in Fig. 11 were used to preload the testing machine when installing the test specimen in the machine and securing it to the upper end plate of the testing machine. The testing was carried out when the machine was in the position shown in Fig. 11. The end plate with the gear drive rested on pins and the opposite end rested on a set of rollers. By this arrangement the frictional torque produced when turning the gears was transmitted through the pins into the base plate on which the testing machine rested, and the test specimen was loaded by axial compression only.

\section{a. Load Cell}

During the buckling tests the load distribution was monitored and the total load was obtained by means of the load cell shown in Fig. 13. It consisted of a seamless, electroformed copper cylinder which was $0.015 \mathrm{in}$. thick, $3.00 \mathrm{in.}$ long and $8.00 \mathrm{in}$. in diameter. Twenty-four foil type strain gages from Micro-Measurements Inc., Serial No. Ma-09-500BH-120 with extra stable characteristics were mounted on the cylinder equally spaced around the circumference. The ones on the inside were directly opposite to those on the outside. It has been shown in reference 14 that the strain measured by this type of arrangement represented very accurately the actual strain in the test shell. The load cell was se cured to the end plate of the testing machine with a thin layer of Devcon. Devcon is a plastic-like 
material in a putty state which hardens in several hours after the addition of a hardening agent.

b. Calibration of the Pick-up

To carry out the imperfection measurements the traversing mechanism was installed in the position shown in Fig. 14 with the shaft protruding through the hole in the end-plate inside the load cell. To carry out the calibration of the pick-up which was installed in a movable support at the end of the protruding shaft, a short shell was cast with Cerrolow into the load cell as shown in Fig. 14. Then the pick-up was moved vertically away from the surface of the shell $0.001 \mathrm{in}$. at a time recording the reading of the digital voltmeter each time. Figure 15 shows a typical calibration curve with the working range and the preset position of the pick-up so indicated. Upon completion of the calibration the pick-up was preset to a position about at the center of the working range. Next the shaft was retracted into the load cell, the short calibration shell was removed and the load cell was ready for the installment of the test shell.

\section{c. Installing the Test Shell}

The test shell was fastened into an end ring with a low melting point alloy Cerrolow and its other end was secured to the load cell in the same manner. After this operation was completed the end ring was secured to the upper end-plate by a thin layer of Devcon. Upon hardening of the Devcon the cylinder was ready for testing. Figure 16 shows the complete cylindrical shell testing configuration. 


\section{d. Initial Imperfection Measurements}

After the installment of the test specimen was completed a full automatic imperfection scan of the shell was performed. The data was recorded both by the IBM card punch and by the xy-plotter. The imperfection measurements on the shells A7, A8, A9, and Al0 were carried out with a pick-up 5/8 in. in diameter, whereas shell A12 was scanned with a smaller pick-up only $1 / 4$ in. in diameter. Because of the integrating properties of the pick-up the axial increments were preset to be $0.50 \mathrm{in.}$ for the large pick-up. In order to avoid unwanted edge effects the initial axial station was located 0.50 in. from the end of the shell which was cast into the load cell. The automatic scanning sequence was started with a counterclockwise circumferential scan at the initial axial station followed by an axial advance of $0.50 \mathrm{in}$. This was followed by a clockwise circumferential scan and the automatic sequence continued with intermittent axial advances and circumferential scans until the full length of the shell was covered. Shells A7, A8, A9, and A10 were 8.00 in. long, so 14 axial advances of $0.50 \mathrm{in}$. each were used. That put the last axial station $0.50 \mathrm{in}$. from the end ring thus avoiding the picking up of any unwanted edge effects. On shell A12 due to the smaller pick-up 30 axial advances of $0.24 \mathrm{in}$. each were used. Upon completion of the initial imperfection measurements the shaft of the scanning device was run back to its initial position. 


\section{e. Monitoring of the Strain Gages}

Before carrying out the buckling tests the gages on the load cell were connected to a 24 channel bridge box which contained 24 Wheatstone bridge circuits. In order to minimize the effect of temperature changes an additional 24 strain gages we re installed on a dummy cylinder and connected to the same bridge box where they formed one of the branches of the individual Wheatstone bridge circuits. The initial zero reading could be adjusted individually through differential shunt balances. The output of the bridge was monitored by a Cimron Digital Voltmeter. By using the stepping switch unit connected between the bridge and the digital voltmeter the readings of the 24 strain gages were recorded automatically by means of an IBM card punch. The total compressive load was computed by averaging the readings of all gages and using a previously determined calibration factor. The calibration of the load cell was carried out using a very accurate Schaevitz dynamometer-type load ring.

\section{f. Buckling Procedure}

Upon completion of the initial imperfection measurements the cylindrical shells were initially loaded to about one-sixth of the expected buckling load and the circumferential load distribution was made as uniform as possible by individually adjusting the four loading screws of the testing machine. This was followed by another complete automatic scanning of the shell surface. The duration of one automatic scan was about $1 / 2$ hour. The strain gages were read before and after the completion of the scan and the load was taken as 
the average of the two readings. The axial load was then increased in small increments by turning the four loading screws simultaneously. After each loading the load distribution was adjusted again. Also after each load increment another complete scan of the shell surface was performed. Thus any change in the shell surface due to the increase in loading was recorded. This was carried out up to about two-thirds of the expected buckling load. After this point the load distribution was no longer adjusted so as to prevent buckling occurring during one of the adjustments. The load was increased in small increments, each load increment followed by a complete scan of the shell surface and the strain gages were monitored until buckling occurred. It should be noted here that due to the working range of $0.125 \mathrm{in}$. of the pick-up used for the imperfection measurements, it was possible to get a complete scan of the postbuckling shapes of the shells tested.

\section{Data Reduction - Main Program}

As described in the previous sections the imperfection measurements were carried out by scanning the surface of the test shells by an inductance-type pick-up. The output of the pick-up was monitored on a digital voltmeter whose readings were recorded at preset intervals by an IBM 526 card punch on cards. Thus, upon completion of each buckling test, there was a set of cards containing the displacement pick-up calibration data in volts and displacements and a set of cards containing the shell displacements given in volts for each scan completed. These displacements were referred to the 
end of the pick-up. The shell displacements were given in volts because they consisted of the readings of the digital voltmeter recorded at preset intervals.

The data reduction of the experimental measurements was done on an IBM 7094 digital computer. Figure 17 shows a flow chart of the main data reduction program. By using an overlay technique it could handle up to $29 \times 97=2813$ experimental points.

a. Best Fit Polynomial to Pick-up Calibration Data

The first step in the data reduction program consisted of fitting a best fit polynomial of the form

$$
\delta=\sum_{n=0}^{N} a_{n} v^{n}
$$

to the working range of the pick-up's calibration data. Figure 15 shows the calibration data of the pick-up used on shell A7 and the corresponding best fit polynomial curve.

The unknown coefficients $a_{n}$ of the polynomial representation were determined by the Method of Least Squares and stored in a common block for later use. As a measure of the accuracy of the

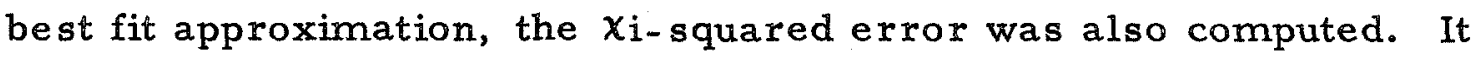
was found that the best possible approximation, that is the approximation for which the $\chi i$-squared error was the least, was obtained for $N=16$.

Next the data reduction program converted the voltage reading s of the initial imperfection scan into displacements in inches 
using the polynomial representation of the calibration curve obtained in the previous step. These displacements were also stored in a common block for later use.

Upon completing the conversion of the data of the initial imperfection scan link ${ }^{(1)}$ was erased from the core except for the results stored in common blocks.

\section{b. Definition of the "Perfect" Cylinder}

In the experimental set up shown in Fig. I a certain misalignment of the shell center line with that of the traversing device was expected. Thus it was necessary to remove from the measured imperfection data the effects of such a misalignment. Also before the harmonic components of the measured imperfection surface could be computed it was necessary to define what was considered the perfect shell. This was accomplished by finding the best fit cylinder to the data of the initial imperfection scan. This was done by the Method of Least Squares by first computing the sum of the squares of the normal distances from the measured points in space to the surface of the assumed best fit cylinder.

$$
S=\sum_{i=1}^{N} d_{i}^{2}=\sum_{i=1}^{N}\left(R_{i}-R\right)^{2}=f\left(X_{1}, Y_{1}, \epsilon_{1}, \epsilon_{2}, R\right)
$$

(See Fig. 18)

Minimizing $S$ with respect to the unknown parameters $X_{1}, Y_{1}, \epsilon_{1}, \epsilon_{2}$ and $R$ yielded five simultaneous, nonlinear algebraic equations in the five unknowns. Assuming that $X_{1}, Y_{1},{ }_{1},{ }_{2}{ }_{2}$ were small, the se 
equations were linearized and solved to determine the new reference axis system and to define the perfect cylinder.

In link ${ }^{(2)}$ the data reduction program computed $X_{1}, Y_{1}, \epsilon_{1}, \epsilon_{2}, R$ and stored them in a common block. Next it took the measured displacements reduced by $\operatorname{link}^{(1)}$ which originally were referred to the end of the scanning pick-up from the common block and recomputed them with respect to the newly found best fit cylinder of radius R. Then the measured displacements, which now represented deviations from the perfect cylinder, were stored in the same common block. Upon completion of this step, link ${ }^{(2)}$ was erased from the core except for the results stored in common blocks.

c. Finding the Harmonic Components of the Measured Imperfection Surface

Three different Fourier representations were used for the measured imperfection surfaces. The full wave representation in the axial direction involved the determination of four sets of harmonic components:

$$
\bar{w}(x, y)=\sum_{m=0}^{N} \sum_{n=0}^{N} A_{m n} \cos m \frac{y}{R} \cos \frac{2 n \pi x}{L}+
$$$$
\sum_{m=1}^{N} \sum_{n=0}^{N} B_{m n} \sin m \frac{y}{R} \cos \frac{2 n \pi x}{L}+
$$$$
\sum_{m=0}^{N} \sum_{n=1}^{N} C_{m n} \cos m \frac{y}{R} \sin \frac{2 n \pi x}{L}+\sum_{m=1}^{N} \sum_{n=1}^{N} D_{m n} \sin m \frac{y}{R} \sin \frac{2 n \pi x}{L}
$$ 
where

$$
\begin{aligned}
& A_{m n}=\frac{2}{\pi L} \int_{0}^{L} \int_{0}^{2 \pi R} \bar{w}(x, y) \cos m \frac{y}{R} \cos \frac{2 n \pi x}{L} d y d x \\
& B_{m n}=\frac{2}{\pi L} \int_{0}^{L} \int_{0}^{2 \pi R} \bar{w}(x, y) \sin m \frac{y}{R} \cos \frac{2 n \pi x}{L} d y d x \\
& \text { L } 2 \pi R \\
& C_{m n}=\frac{2}{\pi L} \int_{0} \int_{0} \bar{w}(x, y) \cos m \frac{y}{R} \sin \frac{2 n \pi x}{L} d y d x \\
& D_{m n}=\frac{2}{\pi L} \int_{0}^{L} \int_{0}^{2 \pi R} \bar{w}(x, y) \sin m \frac{y}{R} \sin \frac{2 n \pi x}{L} d y d x
\end{aligned}
$$

The half wave cosine representation in the axial direction involved the determination of two sets of harmonic components:

$$
\begin{aligned}
\bar{w}(x, y) & =\sum_{m=0}^{N} \sum_{n=0}^{N} A_{m n} \cos m \frac{y}{R} \cos \frac{n \pi x}{L} \\
& +\sum_{m=1}^{N} \sum_{n=0}^{N} B_{m n} \sin m \frac{y}{R} \cos \frac{n \pi x}{L}
\end{aligned}
$$

where 


$$
\begin{aligned}
& A_{m n}=\frac{1}{\pi L} \int_{-L}^{L} \int_{0}^{2 \pi R} \bar{w}(x, y) \cos m \frac{y}{R} \cos \frac{n \pi x}{L} d y d x \\
& B_{m n}=\frac{1}{\pi L} \int_{-L}^{L} \int_{0}^{2 \pi R} \bar{w}(x, y) \sin m \frac{y}{R} \cos \frac{n \pi x}{L} d y d x
\end{aligned}
$$

Finally the half wave sine representation in the axial direction also involved the determination of two sets of harmonic components:

$$
\bar{w}(x, y)=\sum_{m=0}^{N} \sum_{n=1}^{N} C_{m n} \cos m \frac{y}{R} \sin \frac{n \pi x}{L}+\sum_{m=1}^{N} \sum_{n=1}^{N} D_{m n} \sin m \frac{y}{R} \sin \frac{n \pi x}{L}
$$

where

$$
\begin{aligned}
& C_{m n}=\frac{1}{\pi L} \int_{-L}^{L} \int_{0}^{2 \pi} \bar{w}(x, y) \cos m \frac{y}{R} \sin \frac{n \pi x}{L} d y d x \\
& D_{m n}=\frac{1}{\pi L} \int_{-L}^{L} \int_{0}^{2 \pi} \bar{w}(x, y) \sin m \frac{y}{R} \sin \frac{n \pi x}{L} d y d x
\end{aligned}
$$

Separate subroutines were written for the different Fourier representations. The double integrals involved in the determination of the required coefficients were carried out numerically using the trapesoidal rule. Before returning to the main program each subroutine not only printed out the computed Fourier coefficients but also 
punched them on cards. In addition, upon return to the main program, the measured displacements representing the deviations from the defined perfect cylinder were printed out and punched on cards. After the data reduction of the initial imperfection measurements was completed, the program returned to read in the data of the second full scan. The data reduction of the second and of all the subsequent full scans was done using the polynomial representation of. the pick-up calibration obtained during the data reduction of the initial imperfection scan. However the position of the best fit cylinder and with it the definition of the perfect cylinder were recomputed for each scan.

Thus upon completion of the data reduction of the buckling test of a shell by the layered main program the following output has been obtained:

1. A list of the location of the best fit or perfect shell at each load level.

2. The measured shell displacements representing deviations from the perfect shell at each load level both listed and punched on cards.

3. The coefficients of any one of the three Fourier representations at each load level both listed and punched on cards.

6. Data Reduction - Auxiliary Programs

The output from the main data reduction program was examined in different ways. 
a. Three-Dimensional Plots

The cards containing the measured deviations from the perfect shell at different load levels were used as the input to a plotting program. When the proper values were assigned to the built in flags this program prepared three-dimensional plots of the imperfection scans at different load levels by offsetting the origin of the successive circumferential scans by the proper amount along both the $\mathrm{x}$ - and $\mathrm{y}-$ axis. The same program was also used to prepare three-dimensional plots of the growth of imperfections at increasing load levels. This was done by subtracting from the measured shell surface at each load level the measured initial imperfections of the shell before calling the plotting subroutine. The three-dimensional plots were enhanced by using additional points computed by an Aitken interpolation routine.

\section{b. Computing the Growth of Harmonic Components}

The cards containing the computed coefficients of the chosen Fourier representation of the measured shell surfaces at each load level were used as the input to an auxiliary program that computed the growth of each of the coefficients with increasing loading. This was done by subtracting from the coefficients at each load level the corresponding coefficients from the initial scan.

\section{Test Results}

As stated in the introduction, the purpose of this experimental study was to determine the effect of initial imperfections on the buckling load of cylindrical shells under axial compression and to 
find the mechanism by which imperfections reduce the load carrying capacity of the shells. The instrumentation was designed and built to detect such initial imperfections and to trace their growth during the loading process prior to buckling. The results of buckling tests on five cylindrical shells are reported here.

By using electroformed copper shells it was felt that the size of the unintended initial imperfections would be minimized. However all five shells had initial imperfections that were greater than the wall thicknesses of the respective shells as can be seen from Fig. 19 through Fig. 23 which show the initial surveys of shells A7, A8, A9, A10, and A12. The principal Fourier coefficients of these initial surveys, defined as the components whose amplitudes exceeded 5 per cent of the respective wall thicknesses, are summarized in Table II. Table III shows the variation of the "perfect" cylinder with load increments for the five shells tested.

Figures 24 through 26 show the growth of prebuckling deformation of shell A7 at different load levels prior to local buckling. Figure 27 shows the local buckling pattern of shell A7. It confirmed the visual observation made at the time of the buckling test which showed that the local buckling pattern consisted of two isolated waves on the central portion of the shell. The location of the se initial buckles coincided with the most pronounced growth of the prebuckling deformation just prior to the occurrence of the local buckling as shown in Fig. 26. Also at the location of the initial buckles there were very pronounced localized defects in the test 
specimen as can be seen from the initial scan of shell A7, shown in Fig. 19.

Figures 28 through 31 show the growth of prebuckling deformations at the last load level prior to buckling for shells A8, A9, Al0, and A12. Figure 32 shows the local buckling pattern of shell Al0. Figures 33 through 37 show the postbuckling patterns of the five shells tested. It should be mentioned here that for better threedimensional effect these figures should be observed at a rather shallow angle of incidence.

As stated previously, the load distribution was adjusted to be as uniform as possible with the four loading screws of the testing machine. The ajustment was done by equalizing the strain in the load cell at the $45^{\circ}, 135^{\circ}, 225^{\circ}$, and $315^{\circ}$ position. Detailed results of the buckling tests for shells A7, A8, A9, A.10, and A12 are summarized in Table IV. It includes the maximum variation in load distribution near buckling which was kept to about 20 per cent. Figures 38 and 39 show the load distribution on the five shells at the last load level before buckling.

Shells A7 and A.10 failed initially in isolated localized buckles. These localized waves caused the load distribution to fall off in their neighborhood without appreciably affecting the load distribution over the rest of the circumference of the shell. After the initial local buckling the load was increased until general collapse occurred without attempting to adjust the load distribution. General collapse occurred in the same manner as for the other three shells A8, A9, 
and A12 which did not have any initial local buckling, but at a lower value of axial loading.

General collapse consisted of a snap-through which is characteristic of this type of test. In all cases the postbuckling state consisted of 2 to 3 rows of buckles that extended completely a round the circumference. The number of circumferential waves is noted in Table IV.

The three-dimensional plots representing the growth of the prebuckling deformations showed at a glance the general deformation pattern of the shells before buckling. As seen from Fig. 26 and Figs. 28 through 31 there was a very pronounced growth of an imperfection whose half wave length in the axial direction was approximately equal to the length of the shell. The number of circumferential waves of this predominant imperfection mode was approximately equal to the number of circumferential waves in the postbuckled shape. However, the wave length in the axial direction in the postbuckled shape was considerably shorter than the length of the shell as can be seen from Figs. 33 through 37 .

In an attempt to isolate the "critical modal components" defined as those modal components which showed exponential growth close to the critical load - harmonic analyses of the measured imperfection surfaces were carried out at each load level. In order to obtain the mathematical representation that would describe "best" the measured imperfection surfaces different axial expansions were used. Thus the Fourier coefficients of the full wave, the half wave 
sine and the half wave cosine axial representations we re calculated. This involved the computation of approximately 1,400 Fourier coefficients for each complete scan. Detailed investigation of the rate of growth of these coefficients with increasing loading revealed that the consideration should be restricted to coefficients with a growth in excess of one per cent of the respective wall thicknesses at the load level just before buckling. This would include all the coefficients which approach exponential growth at the experimental buckling load. Only about 40 of the computed Fourier coefficients showed significant growth rate and only about 20 of the se showed exponential growth. All the coefficients that were investigated are summarized in Table V. Figures 40 through 44 show the rate of growth of three such "critical modal components" for shells A7, A8, $A 9, A 10$, and $A 12$ respectively. As can be seen from the se figures most of the se curves representing the experimentally measured growth rates approach a horizontal tangent at the experimental buckling load. The same characteristic curves were obtained for some of the other coefficients. However, most of the coefficients that approach a horizontal tangent at the experimental buckling load had similar circumferential and axial wave numbers. 


\section{COMPARISON WITH THEORY}

By tracing the growth of individual modal components of the measured imperfection surfaces during the loading process prior to buckling it was discovered that for each shell tested several different modal components seemed to approach a horizontal tangent at about the experimental buckling load. It was, however, impossible to ascertain the exact value of loading at the respective points of horizontal tangency just from observation of the given experimental data.

In considering the available theoretical methods it was found that none of them were quite general enough to be used directly with the measured experimental values. Koiter (Ref. 9) has presented an analytical procedure for obtaining the role of imperfections in imperfection sensitive structures. Later, working with an imperfection in the form of the radial displacement component of the axisymmetric buckling mode $\bar{W}=\mu \cos \left(q_{0} x / R\right)$, he was able to show (Ref. 10) that such an imperfection would reduce the buckling load of a cylindrical shell under axial compression to as low as one-third of the classical value for values of $\mu$ only a fraction of the shell thickness. However, he considered axisymmetric imperfections only.

In general, any radial imperfection pattern of the shell can be represented by a double Fourier series in the axial and circumferential coordinates. Thus in an effort to gain insight into the effects of the nonlinear interactions of several buckling modes an approximate solution of the nonlinear Donnell type shell equations was obtained with the initial radial imperfection being represented by only three 
terms of the double Fourier series, one axisymmetric and two asymmetric ones. The approximate solution of the full nonlinear equations was obtained as follows:

1. The compatibility equation was solved exactly for the stress function $F$ in terms of the assumed radial displacement $W$ and the measured imperfection $\bar{W}$. This guaranteed that a kinematically admissible displacement field would be associated with the solution of the second equation, the condition of equilibrium. 2. The equation of equilibrium was solved approximately by substituting therein $F, W$, and $\bar{W}$ and then applying the Galerkin procedure.

It should be mentioned here that a similar method of solution has been used by Hutchinson (Ref. 12). However he restricted his imperfections to the form of a linear buckling mode of the cylindrical shell. In the following solution this restriction was removed.

\section{Donnell's Shell Equations}

Assuming that the radial displacement $W$ is positive outward and that the membrane stress resultants can be obtained from an Airy stress function $F$ as $N_{x}=F_{y_{y y}}, N_{y}=F, x x$ and $N_{x y}=-F$, $x y$, then Donnell's equations for an imperfection cylindrical shell (Ref. 13) can be written as: 


$$
\begin{aligned}
& \frac{1}{E t} \nabla^{4} F-\frac{1}{R} W,_{x x}+\frac{1}{2} L(W, W+2 \bar{W})=0 \\
& \frac{E t^{3}}{12\left(1-\nu^{2}\right)} \nabla^{4} W+\frac{1}{R} F,_{x x}-L(F, W+\bar{W})=0
\end{aligned}
$$

where the nonlinear operator $L$ is defined by

$$
L(S, T)=S,{ }_{x x} T,_{y y}-2 S,{ }_{x y} T,_{x y}+S,{ }_{y y} T, x x
$$

and $\nabla^{4}$ is the two-dimensional biharmonic operator. See Fig. 45 for notation.

\section{Nonlinear Buckling Equations}

The solution assumes initial imperfection shapes represented by

$$
\bar{W}=\bar{\xi}_{1} t \cos i \bar{x}+\bar{\xi}_{2} t \cos k \bar{x} \cdot \cos \ell \bar{y}+\bar{\xi}_{3} t \sin k \bar{x} \cdot \cos \ell \bar{y}
$$

The equilibrium state of the axially loaded cylinder will be expressed as :

$$
\begin{aligned}
& W=\frac{\nu}{E} \sigma t+w \\
& F=-\frac{1}{2} \sigma t y^{2}+f
\end{aligned}
$$

where the terms added to $w$ and $f$ constitute the membrane prebuckling solution for the perfect shell. Further, w will be assumed as

$$
w=\xi_{1} t \cos i \bar{x}+\xi_{2} t \cos k \bar{x} \cdot \cos \ell \bar{y}+\xi_{3} t \sin k \bar{x} \cdot \cos l \bar{y}(2.4)
$$


In the following approximate solution the effect of boundary conditions will be neglected. Although in some instances the shell buckling may be characterized by certain end conditions, as has been shown by Hoff (Ref. 5) and Kobayashi (Ref. 6), in this solution only the effect of initial imperfections on the buckling load is of interest. Substituting the assumed form of $W$ and $\bar{W}$ into the compatibility equation $(1.1)$ yields

$\frac{1}{\bar{E} t}\left(F,_{x x x x}+2 F,_{x x y y}+F,_{y y y y}\right)=K_{1} \cos 2 k \bar{x}+K_{2} \cos \bar{i} \bar{x}+K_{3} \cos 2 \ell \bar{y}$

$+\mathrm{K}_{4} \sin 2 \mathrm{k} \overline{\mathrm{x}}+\mathrm{K}_{5} \cos \mathrm{k} \overline{\mathrm{x}} \cdot \cos \ell \overline{\mathrm{y}}+\mathrm{K}_{6} \sin \mathrm{k} \overline{\mathrm{x}} \cdot \cos \ell \overline{\mathrm{y}}$

$+K_{7} \cos (i+k) \bar{x} \cdot \cos \ell \bar{y}+K_{8} \cos (i-k) \bar{x} \cdot \cos \ell \bar{y}$

$+\mathrm{K}_{9} \sin (\mathrm{i}+\mathrm{k}) \overline{\mathrm{x}} \cdot \cos \ell \overline{\mathrm{y}}+\mathrm{K}_{10} \sin (\mathrm{i}-\mathrm{k}) \overline{\mathrm{x}} \cdot \cos \ell \overline{\mathrm{y}}$

where the constants $\mathrm{K}_{1}, \mathrm{~K}_{2}, \ldots, \mathrm{K}_{10}$ are defined in Appendix A.

Since the boundary conditions of the finite shell will be neglected, therefore only a particular solution of equation (2.5) needs to be considered. To obtain such a particular solution let

$$
F=-\frac{1}{2} \sigma t y^{2}+f_{p}
$$

where 
$f_{p}=A \cos 2 k \bar{x}+B \cos i \bar{x}+C \cos 2 \ell \bar{y}+D \sin 2 k \bar{x}+E \cos k \bar{x} \cdot \cos l \bar{y}$

$+F \sin k \bar{x} \cdot \cos l \bar{y}+G \cos (i+k) \bar{x} \cdot \cos l \bar{y}+H \cos (i-k) \bar{x} \cdot \cos l \bar{y}$

$+I \sin (i+k) \bar{x} \cdot \cos l \bar{y}+J \sin (i-k) \bar{x} \cdot \cos l_{\bar{y}}$

The unknown coefficients $A, B, \ldots, J$ are determined by substituting the assumed $F$ into equation (2.5) and equating coefficients of like terms. They are written out in detail in Appendix A.

Substituting the assumed form for $W, \bar{W}$ and the computed particular solution for $F$ as given by equations (2.1), (2.2) and (2.3) into the equilibrium equation (1.2) yields the "error" $\varepsilon_{\mathrm{N}}=\varepsilon_{\mathrm{N}}$ $\left(\xi_{1}, \xi_{2}, \xi_{3}, \bar{x}, \bar{y}\right)$. Using Galerkin's idea of minimization of the "error" with respect to a set of functions leads to a system of three nonlinear algebraic equations in the three unknowns $\xi_{1}, \xi_{2}, \xi_{3}$. Here these equations are obtained from the following integ rals

$$
\begin{aligned}
& \int_{0}^{2 \pi} \int_{0}^{2 \pi} \varepsilon_{N}\left(\xi_{1}, \xi_{2}, \xi_{3}, \bar{x}, \bar{y}\right) \cdot \cos i \bar{x} d \bar{x} d \bar{y}=0 \\
& \int_{0}^{2 \pi} \int_{0}^{2 \pi} \varepsilon_{N}\left(\xi_{1}, \xi_{2}, \xi_{3}, \bar{x}, \bar{y}\right) \cdot \cos k \bar{x} \cdot \cos l \bar{y} d \bar{x} d \bar{y}=0
\end{aligned}
$$


35

$$
\int_{0}^{2 \pi} \int_{0}^{2 \pi} \varepsilon_{N^{(}}\left(\xi_{1}, \xi_{2}, \xi_{3}, \bar{x}, \bar{y}\right) \cdot \sin k \bar{x} \cdot \cos l \bar{y} d \bar{x} d \bar{y}=0
$$

Imposing the restriction $k=\frac{i}{2}$, carrying out the indicated integraltrons and introducing the following nondimensional parameters

$$
\begin{aligned}
\alpha_{A}^{2}=i^{2} \frac{R t}{2 c}\left(\frac{2 \pi}{L}\right)^{2} & \alpha_{2}^{2}=(i-k)^{2} \frac{R t}{2 c}\left(\frac{2 \pi}{L}\right)^{2} \\
\alpha^{2}=k^{2} \frac{R t}{2 c}\left(\frac{2 \pi}{L}\right)^{2} & \alpha_{3}^{2}=\frac{i^{4}}{k^{2}} \frac{R t}{2 c}\left(\frac{2 \pi}{L}\right)^{2} \\
\alpha_{1}^{2}=(i+k)^{2} \frac{R t}{2 c}\left(\frac{2 \pi}{L}\right)^{2} & \beta^{2}=l^{2} \frac{R t}{2 c}\left(\frac{1}{R}\right)^{2} \\
\lambda=c \frac{R}{t} \frac{\sigma}{E} & c=\sqrt{3\left(1-\nu^{2}\right)}
\end{aligned}
$$

yields the following equilibrium equations:

$$
\begin{aligned}
& \xi_{1}\left\{\frac{1}{2}\left(\alpha_{A}^{2}+\frac{1}{\alpha_{A}^{2}}\right)-\lambda\right\}+\frac{c}{16}\left\{\frac{\beta^{2}}{\alpha^{2}}\right\}\left[\bar{\xi}_{2} \xi_{2}-\bar{\xi}_{3} \xi_{3}+\frac{1}{2}\left(\xi_{2}^{2}-\xi_{3}^{2}\right)\right] \\
& +\frac{c}{4}\left\{\frac{\alpha^{2} \beta^{2}}{\left(\alpha^{2}+\beta^{2}\right)^{2}}\right\}\left[\left(\xi_{2}+\bar{\xi}_{2}\right) \xi_{2}-\left(\xi_{3}+\bar{\xi}_{3}\right) \xi_{3}\right] \\
& +\frac{c}{2}\left\{\frac{\alpha_{A}^{2} \beta^{4}}{\left(\alpha_{1}^{2}+\beta^{2}\right)^{2}}+\frac{\alpha_{A}^{2} \beta^{4}}{\left(\alpha_{2}^{2}+\beta^{2}\right)^{2}}\right\}\left\{\left(\xi_{2}+\bar{\xi}_{2}\right)\left[\left(\xi_{1}+\bar{\xi}_{1}\right) \xi_{2}+\bar{\xi}_{2} \xi_{1}\right]\right. \\
& \left.\quad+\left(\xi_{3}+\bar{\xi}_{3}\right)\left[\left(\xi_{1}+\bar{\xi}_{1}\right) \xi_{3}+\bar{\xi}_{3} \xi_{1}\right]\right\}=\lambda \bar{\xi}_{1}
\end{aligned}
$$


36

$$
\begin{aligned}
\xi_{2}\left\{\frac{1}{2}\left[\frac{\left(\alpha^{2}+\beta^{2}\right)^{2}}{\alpha^{2}}+\frac{\alpha^{2}}{\left(\alpha^{2}+\beta^{2}\right)^{2}}\right]-\lambda\right\}+2 c\left\{\frac{\alpha^{2} \beta^{2}}{\left(\alpha^{2}+\beta^{2}\right)^{2}}\right\}\left[2\left(\xi_{1}+\bar{\xi}_{1}\right) \xi_{2}+\bar{\xi}_{2} \xi_{1}\right] \\
\quad+\frac{c}{2}\left\{\frac{\beta^{2}}{\alpha^{2}}\right\}\left(\xi_{2}+\bar{\xi}_{2}\right) \xi_{1}+\frac{c^{2}}{4}\left\{\frac{\beta^{4}}{\alpha^{2}}\right\}\left\{\left(\xi_{3}+\bar{\xi}_{3}\right)\left[\left(\xi_{2}+\bar{\xi}_{2}\right) \xi_{3}+\bar{\xi}_{3} \xi_{2}\right]\right. \\
\left.+\left(\xi_{2}+\bar{\xi}_{2}\right)\left[\bar{\xi}_{2} \xi_{2}-\bar{\xi}_{3} \xi_{3}+\frac{1}{2}\left(\xi_{2}^{2}-\xi_{3}^{2}\right)\right]\right\} \\
\quad+\frac{c^{2}}{4}\left\{\alpha^{2}\right\}\left[\bar{\xi}_{2} \xi_{2}+\bar{\xi}_{3} \xi_{3}+\frac{1}{2}\left(\xi_{2}^{2}+\xi_{3}^{2}\right)\right]\left(\xi_{2}+\bar{\xi}_{2}\right) \\
\quad+\frac{c^{2}}{2}\left\{\frac{\alpha_{3}^{2} \beta^{4}}{\left(\alpha_{1}^{2}+\beta^{2}\right)^{2}}+\frac{\alpha_{3}^{2} \beta^{4}}{\left(\alpha_{2}^{2}+\beta^{2}\right)^{2}}\right\}\left[\left(\xi_{1}+\bar{\xi}_{1}\right) \xi_{2}+\bar{\xi}_{2} \xi_{1}\right]\left(\xi_{1}+\bar{\xi}_{1}\right)=\lambda \bar{\xi}_{2}
\end{aligned}
$$

$$
\begin{aligned}
\xi_{3}\left\{\frac{1}{2}\left[\frac{\left(\alpha^{2}+\beta^{2}\right)^{2}}{\alpha^{2}}+\frac{\alpha^{2}}{\left(\alpha^{2}+\beta^{2}\right)^{2}}\right]-\lambda\right\}-2 c\left\{\frac{\alpha^{2} \beta^{2}}{\left(\alpha^{2}+\beta^{2}\right)^{2}}\right\}\left[2\left(\xi_{1}+\bar{\xi}_{1}\right) \xi_{3}+\bar{\xi}_{3} \xi_{1}\right] \\
-\frac{c}{2}\left\{\frac{\beta^{2}}{\alpha^{2}}\right\}\left(\xi_{3}+\bar{\xi}_{3}\right) \xi_{1}-\frac{c^{2}}{4}\left\{\frac{\beta^{4}}{\alpha^{2}}\right\}\left\{\left(\xi_{3}+\bar{\xi}_{3}\right)\left[\bar{\xi}_{2} \xi_{2}-\bar{\xi}_{3} \xi_{3}+\frac{1}{2}\left(\xi_{2}^{2}-\xi_{3}^{2}\right)\right]\right. \\
\left.-\left(\xi_{2}+\bar{\xi}_{2}\right)\left[\left(\xi_{2}+\bar{\xi}_{2}\right) \xi_{3}+\bar{\xi}_{3} \xi_{2}\right]\right\}+\frac{c^{2}}{4}\left\{\alpha^{2}\right\}\left[\bar{\xi}_{2} \xi_{2}+\bar{\xi}_{3} \xi_{3}+\frac{1}{2}\left(\xi_{2}^{2}+\xi_{3}^{2}\right)\right]\left(\xi_{3}+\bar{\xi}_{3}\right) \\
+\frac{c^{2}}{2}\left\{\frac{\alpha_{3}^{2} \beta^{4}}{\left(\alpha_{1}^{2}+\beta^{2}\right)^{2}}+\frac{\alpha_{3}^{2} \beta^{4}}{\left(\alpha_{2}^{2}+\beta^{2}\right)^{2}}\right\}\left[\left(\xi_{1}+\bar{\xi}_{1}\right) \xi_{3}+\bar{\xi}_{3} \xi_{1}\right]\left(\xi_{1}+\bar{\xi}_{1}\right)=\lambda \bar{\xi}_{3}
\end{aligned}
$$


A solution of the se "nonlinear equations" would yield the equilibrium configuration of the finite shell as a function of $\lambda$. However for $\bar{\xi}_{1}$ and $\bar{\xi}_{2}$ small enough the essential character of the shell in the prebuckling and the immediate postbuckling configuration is retained if the terms of the order $\bar{\xi}^{2}, \bar{\xi}^{2} \xi$ and $\xi^{3}$ are omitted from the previous equations. Thus the following system of simplified equations is obtained:

$$
\begin{aligned}
& \xi_{1}\left(C_{1}-\lambda\right)+C_{2}\left[\bar{\xi}_{2} \xi_{2}-\bar{\xi}_{3} \xi_{3}+\frac{1}{2}\left(\xi_{2}^{2}-\xi_{3}^{2}\right)\right]+C_{3}\left[\left(\xi_{2}+\bar{\xi}_{2}\right) \xi_{2}-\left(\xi_{3}+\xi_{3} \bar{\xi}_{3}\right]\right. \\
& \xi_{2}\left(C_{4}-\lambda\right)+8 C_{3}\left[2\left(\xi_{1}+\bar{\xi}_{1}\right) \xi_{2}+\bar{\xi}_{2} \xi_{1}\right]+8 C_{2}\left(\xi_{2}+\bar{\xi}_{2}\right) \xi_{1}=\lambda \bar{\xi}_{2} \\
& \xi_{3}\left(C_{4}-\lambda\right)-8 C_{3}\left[2\left(\xi_{1}+\bar{\xi}_{1}\right) \xi_{3}+\bar{\xi}_{3} \xi_{1}\right]-8 C_{2}\left(\xi_{3}+\bar{\xi}_{3}\right) \xi_{1}=\lambda \bar{\xi}_{3}
\end{aligned}
$$

whe re

$$
\begin{array}{ll}
C_{1}=\frac{1}{2}\left[\alpha_{A}^{2}+\frac{1}{\alpha A^{2}}\right] & C_{2}=\frac{c}{16} \frac{\beta^{2}}{\alpha^{2}} \\
C_{3}=\frac{c}{4} \frac{\alpha^{2} \beta^{2}}{\left(\alpha^{2}+\beta^{2}\right)^{2}} & C_{4}=\frac{1}{2}\left[\frac{\left(\alpha^{2}+\beta^{2}\right)}{\alpha^{2}}+\frac{\alpha^{2}}{\left(\alpha^{2}+\beta^{2}\right)^{2}}\right]
\end{array}
$$


38.

3. Buckling of a Perfect Shell: $\bar{\xi}_{1}=\bar{\xi}_{2}=\bar{\xi}_{3}=0$

In this case the governing equations become

$$
\begin{aligned}
& \xi_{1}\left(C_{1}-\lambda\right)+\left(\frac{1}{2} C_{2}+C_{3}\right)\left(\xi_{2}^{2}-\xi_{3}^{2}\right)=0 \\
& \xi_{2}\left\{C_{4}-\lambda+8\left(C_{2}+2 C_{3}\right) \xi_{1}\right\}=0 \\
& \xi_{3}\left\{C_{4}-\lambda-8\left(C_{2}+2 C_{3}\right) \xi_{1}\right\}=0
\end{aligned}
$$

If $\mathrm{C}_{1} \geq \mathrm{C}_{4}$, there is no deformation of a perfect shell in the buckling modes $\left(\xi_{1}=\xi_{2}=\xi_{3}=0\right)$ until $\lambda$ reaches $C_{4}$. Then the coefficients of $\xi_{2}$ and $\xi_{3}$ in equations. (3.2) and (3.3) vanish and a bifurcation of the solution in the $\xi_{2}$ or $\xi_{3}$ mode will occur. Since $\bar{\xi}_{1}=0, \xi_{1}$ can be either positive or negative. Suppose $\xi_{3}=0$, then following bifurcation $\lambda$ will decrease with deformation occurring in the $\xi_{2}$ as well as the negative axisymmetric mode $\xi_{1}$. However if $\xi_{2}=0$, then following bifurcation $\lambda$ will decrease with deformation occurring in the $\xi_{3}$ and in the positive axisymmetric mode $\xi_{1}$. In either case the maximum value of $\lambda$ attained is at $\lambda=C_{4}$.

If $\mathrm{C}_{1}<\mathrm{C}_{4}$, there is no deformation of a perfect shell in the buckling modes $\left(\xi_{1}=\xi_{2}=\xi_{3}=0\right)$ until $\lambda$ reaches $C_{1}$. With $\lambda$ remaining at $\mathrm{C}_{1}$, deformation can occur in the axisymmetric mode; and since $\bar{\xi}_{1}=0, \xi_{1}$ can be either positive or negative. If $\xi_{1}$ attains the value $-\left(C_{4}-C_{1}\right) / 8\left(C_{2}+2 C_{3}\right)$ then the coefficient of $\xi_{2}$ in equation (3.2) vanishes and a bifurcation of the solution in the $\xi_{2}$ mode occurs. 
The bifurcated solution corresponds to decreasing values of $\lambda$ with deformation in both the $\xi_{1}$ and $\xi_{2}$ modes. Similarly if the axisymmetric deformation is such that $\xi_{1}$ attains the value $\left(C_{4}-C_{1}\right) / 8$ $\left(\mathrm{C}_{2}+2 \mathrm{C}_{3}\right)$ then the coefficient of $\xi_{3}$ in equation (3.3) vanishes and $\lambda$ decreases with deformation in both the $\xi_{1}$ and $\xi_{3}$ modes. In either case the maximum value of $\lambda$ attained is at $\lambda=C_{1}$. The behavior of the perfect shell is shown in Fig. 46.

4. Buckling of a Shell with Axisymmetric Imperfection:

$$
\bar{\xi}_{1} \neq 0, \bar{\xi}_{2}=\bar{\xi}_{3}=0
$$

In this case the governing equations become:

$$
\begin{aligned}
& \xi_{1}\left(C_{1}-\lambda\right)+\left(\frac{1}{2} C_{2}+C_{3}\right)\left(\xi_{2}^{2}-\xi_{3}^{2}\right)=\lambda \bar{\xi}_{1} \\
& \xi_{2}\left\{C_{4}-\lambda+8\left(C_{2}+2 C_{3}\right) \xi_{1}+16 C_{3} \bar{\xi}_{1}\right\}=0 \\
& \xi_{3}\left\{C_{4}-\lambda-8\left(C_{2}+2 C_{3}\right) \xi_{1}-16 C_{3} \bar{\xi}_{1}\right\}=0
\end{aligned}
$$

Now if the imperfection is purely axisymmetric then the prebuckling deformation is also purely axisymmetric. Hence $\xi_{2}=\xi_{3}=0$ which satisfies equations (4.2) and (4.3) identically and from equation (4.1)

$$
\xi_{1}=\frac{\lambda}{C_{1}-\lambda} \bar{\xi}_{1}
$$

until there is a bifurcation of the solution in one of the asymmetric modes. 
For $\bar{\xi}_{1}$ negative the prebuckling axisymmetric deformation $\xi_{1}$ will be negative and bifurcation into the asymmetric mode will occur when the coefficient of $\xi_{2}$ in equation (4.2) vanishes. That is when

$$
\left\{C_{4}-\lambda+8\left(C_{2}+2 C_{3}\right) \xi_{1}+16 C_{3} \bar{\xi}_{1}\right\}=0
$$

which is the equation of a straight line in the $\lambda \xi_{1}$ - plane. Following bifurcation the value of $\lambda$ decreases with deformation occurring in both the axisymmetric mode $\xi_{1}$ and the asymmetric mode $\xi_{2}$.

For $\bar{\xi}_{1}$ positive the prebuckling axisymmetric deformation $\xi_{1}$ will be positive and bifurcation into the asymmetric mode will occur when the coefficient of $\xi_{3}$ in equation (4.3) vanishes. That is when

$$
\left\{C_{4}-\lambda-8\left(C_{2}+2 C_{3}\right) \xi_{1}-16 C_{3} \bar{\xi}_{1}\right\}=0
$$

which is the equation of another straight line in the $\lambda \xi_{1}$ - plane. Following bifurcation the value of $\lambda$ decreases with deformation occurring in both the axisymmetric mode $\xi_{1}$ and the asymmetric mode $\xi_{3}$. This behavior of the shell is shown in Fig. 47.

The maximum value of $\lambda_{1}$ denoted by $\lambda_{M}$, occurs at the bifurcation point and can be solved for explicitely by eliminating $\xi_{1}$ between equations (4.4) and (4.5). Its value is found to be

$$
\lambda_{M}=\frac{B}{2}-\frac{1}{2} \sqrt{B^{2}-4 D}
$$


where

$$
\begin{aligned}
& B=C_{1}+C_{4}+8 C_{2} \bar{\xi}_{1} \\
& D=C_{1}\left(C_{4}-16 C_{3} \overline{\xi_{1}}\right)
\end{aligned}
$$

5. Buckling of a Shell with Both Axisymmetric and Asymmetric Imperfections: $\bar{\xi}_{1} \neq 0$ and $\bar{\xi}_{2} \neq 0$, $\bar{\xi}_{3}=0$ or $\bar{\xi}_{2}=0, \bar{\xi}_{3} \neq 0$

If $\bar{\xi}_{1} \neq 0, \overline{\xi_{2}} \neq 0$ and $\bar{\xi}_{3}=0$ then the governing equations become:

$$
\begin{aligned}
& \xi_{1}\left(C_{1}-\lambda\right)+C_{2}\left[\bar{\xi}_{2} \xi_{2}+\frac{1}{2}\left(\xi_{2}^{2}-\xi_{3}^{2}\right)\right]+C_{3}\left[\left(\xi_{2}+\bar{\xi}_{2}\right) \xi_{2}-\xi_{3}^{2}\right]=\lambda \bar{\xi}_{1} \\
& \xi_{2}\left(C_{4}-\lambda\right)+8 C_{3}\left[2\left(\xi_{1}+\bar{\xi}_{1}\right) \xi_{2}+\bar{\xi}_{2} \xi_{1}\right]+8 C_{2}\left(\xi_{2}+\bar{\xi}_{2}\right) \xi_{1}=\lambda \bar{\xi}_{2} \\
& \xi_{3}\left\{C_{4}-\lambda-16 C_{3}\left(\xi_{1}+\bar{\xi}_{1}\right)-8 C_{2} \xi_{1}\right\}=0
\end{aligned}
$$

In this case deformation occurs in both the $\xi_{1}$ and $\xi_{2}$ modes for any nonzero values of $\lambda$. Nonzero values of $\xi_{3}$ can occur only if the coefficient of $\xi_{3}$ in equation (5.3) vanishes. Assuming that such does not occur then $\xi_{3}=0$. This satisfies equation (5.3) identically and reduces the other two equations to:

$$
\xi_{1}\left(C_{1}-\lambda\right)+C_{2}\left(\bar{\xi}_{2} \xi_{2}+\frac{1}{2} \xi_{2}^{2}\right)+C_{3}\left(\xi_{2}+\bar{\xi}_{2}\right) \xi_{2}=\lambda \bar{\xi}_{1}
$$




$$
\xi_{2}\left(C_{4}-\lambda\right)+8 C_{3}\left[2\left(\xi_{1}+\bar{\xi}_{1}\right) \xi_{2}+\bar{\xi}_{2} \xi_{1}\right]+8 C_{2}\left(\xi_{2}+\bar{\xi}_{2}\right) \xi_{1}=\lambda \bar{\xi}_{2}
$$

The trace of the general solution curve in the $\lambda \xi_{2}$ - plane can be obtained by eliminating $\xi_{1}$ between equations (5.4) and (5.5). This yields

$$
A_{1} \lambda^{2}+B_{1} \lambda+D_{1}=0
$$

where

$$
\begin{aligned}
A_{1}= & \xi_{2}+\bar{\xi}_{2} \\
B_{1}= & \left(C_{1}+C_{4}-8 C_{2} \bar{\xi}_{1}\right) \xi_{2}+C_{1} \bar{\xi}_{2}-8\left(C_{2}+C_{3}\right) \bar{\xi}_{1} \bar{\xi}_{2} \\
D_{1}= & {\left[C_{1} C_{4}+16 C_{1} C_{3} \bar{\xi}_{1}-8\left(C_{2}+C_{3}\right)^{2} \bar{\xi}_{2}^{2}\right] \cdot \xi_{2} } \\
& -\left(C_{2}+2 C_{3}\right)\left[12\left(C_{2}+C_{3}\right) \bar{\xi}_{2} \xi_{2}^{2}+4\left(C_{2}+2 C_{3}\right) \xi_{2}^{3}\right]
\end{aligned}
$$

But this is just a standard quadratic equation whose solution can be written down immediately as:

$$
\lambda=\frac{B_{1}}{2 A_{1}} \pm \frac{1}{2 A_{1}} \sqrt{B_{1}^{2}-4 A_{1} D_{1}}=f\left(\xi_{2}, \bar{\xi}_{1}, \bar{\xi}_{2}, \alpha, \beta, c\right)
$$

Here the negative sign must be used for $\bar{\xi}_{2}$ positive and the positive sign for $\bar{\xi}_{2}$ negative. 
A maximum value of $\lambda$ (if it occurs) is associated with $d \lambda / d \xi_{2}=0$. This condition along with equation $(5.6)$ can be used to solve for $\lambda_{M}$. The result is analogous to equation (4.7) but considerably more complicated. Hence it is more convenient to obtain $\lambda_{M}$ from the $\lambda$ vs. $\xi_{2}$ plot using equation (5.8). This behavior of the shell is shown in Fig. 48 .

If $\bar{\xi}_{1} \neq 0, \bar{\xi}_{2}=0$ and $\bar{\xi}_{3} \neq 0$ then the governing equations become:

$$
\begin{aligned}
& \xi_{1}\left(C_{1}-\lambda\right)+C_{2}\left[-\bar{\xi}_{3} \xi_{3}+\frac{1}{2}\left(\xi_{2}^{2}-\xi_{3}^{2}\right)\right]+C_{3}\left[\xi_{2}^{2}-\left(\xi_{3}+\bar{\xi}_{3}\right) \xi_{3}\right]=\lambda \bar{\xi}_{1} \\
& \xi_{2}\left\{C_{4}-\lambda+16 C_{3}\left(\xi_{1}+\bar{\xi}_{1}\right)+8 C_{2} \xi_{1}\right\}=0 \\
& \xi_{3}\left(C_{4}-\lambda\right)-8 C_{3}\left[2\left(\xi_{1}+\bar{\xi}_{1}\right) \xi_{3}+\bar{\xi}_{3} \xi_{1}\right]-8 C_{2}\left(\xi_{3}+\bar{\xi}_{3}\right) \xi_{1}=\lambda \bar{\xi}_{3}
\end{aligned}
$$

Here deformation occurs in both the $\xi_{1}$ and $\xi_{3}$ modes for any nonzero value of $\lambda$. Nonzero values of $\xi_{2}$ can occur only if the coefficient of $\xi_{2}$ in equation (5.10) vanishes. Assuming that such does not occur then $\xi_{2}=0$. This satisfies equation (5.10) identically and reduces the other two equations to:

$$
\begin{aligned}
& \xi_{1}\left(C_{1}-\lambda\right)-C_{2}\left(\bar{\xi}_{3} \xi_{3}+\frac{1}{2} \xi_{3}^{2}\right)-C_{3}\left(\xi_{3}+\bar{\xi}_{3}\right) \xi_{3}=\lambda \bar{\xi}_{1} \\
& \xi_{3}\left(C_{4}-\lambda\right)-8 C_{3}\left[2\left(\xi_{1}+\bar{\xi}_{1}\right) \xi_{3}+\bar{\xi}_{3} \xi_{1}\right]-8 C_{2}\left(\xi_{3}+\bar{\xi}_{3}\right) \xi_{1}=\lambda \bar{\xi}_{3}
\end{aligned}
$$


The trace of the general solution curve in the $\lambda \xi_{3}$ - plane can be obtained by eliminating $\xi_{1}$ between equations (5.12) and (5.13). This yields

$$
A_{2} \lambda^{2}+B_{2} \lambda+D_{2}=0
$$

where

$$
\begin{aligned}
A_{2}= & \xi_{3}+\bar{\xi}_{3} \\
B_{2}= & \left(C_{1}+C_{4}+8 C_{2} \bar{\xi}_{1}\right) \xi_{3}+C_{1} \bar{\xi}_{3}+8\left(C_{2}+C_{3} \bar{\xi}_{1} \bar{\xi}_{3}\right. \\
D_{2}= & {\left[C_{1} C_{4}-16 C_{1} C_{3} \bar{\xi}_{1}-8\left(C_{2}+C_{3}\right)^{2} \bar{\xi}_{2}^{2}\right] \xi_{3} } \\
& -\left(C_{2}+2 C_{3}\right)\left[12\left(C_{2}+C_{3} \bar{\xi}_{3} \xi_{3}^{2}+4\left(C_{2}+2 C_{3}\right) \xi_{3}^{3}\right]\right.
\end{aligned}
$$

The solution of this standard quadratic equation can be written down immediately as:

$$
\lambda=\frac{\mathrm{B}_{2}}{2 \mathrm{~A}_{2}} \pm \frac{1}{2 \mathrm{~A}_{2}} \sqrt{\mathrm{B}_{2}^{2}-4 \mathrm{~A}_{2} \mathrm{D}_{2}}=\mathrm{g}\left(\xi_{3}, \bar{\xi}_{1}, \bar{\xi}_{2}, \alpha, \beta, c\right)
$$

The negative sign must be used for $\bar{\xi}_{3}$ positive and the positive sign for $\bar{\xi}_{3}$ negative.

A maximum value of $\lambda$ (if it occurs) is associated with $\mathrm{d} \lambda / \mathrm{d} \xi_{3}=0$. This condition along with equation (5.16) could be used to 
solve for $\lambda_{M}$. However, as pointed out earlier, it was found more convenient to obtain $\lambda_{M}$ from the $\lambda$ vs. $\xi_{3}$ plot using equation (5. 16). This behavior of the shell is shown in Fig. 48 .

As expected, the introduction of the additional asymmetric imperfection decreases the load carrying capacity of the shell even further. Thus the $\lambda_{M}$ obtained from the $\lambda$ vs. $\xi_{2}$ plot using. equation (5.8) or from the $\lambda$ vs. $\xi_{3}$ plot using equation (5.16) for a shell with both axisymmetric and asymmetric imperfections will always be less than the $\lambda_{M}$ computed from equation (4.7) for a shell with axisymmetric imperfection only.

\section{Numerical Results}

Equations (5.8) and (5.16) were programmed on the IBM 7094 and used in the search for the "pair of critical modal components", defined as that combination of one axisymmetric and one asymmetric imperfection component which gave the lowest value for ${ }{ }_{M}$.

In deriving the buckling equations (2.11)-(2.13) it was necessary to impose the condition $k=i / 2$ otherwise all the quad. ratic terms in $\xi_{1}, \xi_{2}$, and $\xi_{3}$ would vanish identically and the resulting buckling equations would describe a system with stable postbuckled states which are known to be insensitive to initial imperfections. Thus choosing an axisymmetric imperfection with wave number $i$ automatically fixed the axial wave number $k$ of the asymmetric imperfection. Then in order to minimize the asymmetric buckling load $\lambda=C_{4}$ the circumferential wave number $l$ was computed from 


$$
\alpha^{2}+\beta^{2}-\alpha=0
$$

or solving for $l$ :

$$
l=R \sqrt{k\left(\frac{2 \pi}{L}\right)^{2}\left(\frac{L}{2 \pi} \sqrt{\frac{2 c}{R t}}-k\right)}
$$

with the restriction that for a positive, nonzero $l$

$$
k<\frac{L}{2 \pi} \sqrt{\frac{2 c}{R t}}=i_{c l}
$$

where

$$
\begin{aligned}
i_{c l}= & \text { wave number of the classical axisymmetric buckling } \\
& \text { mode }
\end{aligned}
$$

The computation of the wave numbers is summarized in Table VI for the shells tested. In actual use $l$ was rounded to the nearest integer.

When combining the harmonic components of the experimentally measured imperfection surfaces into pairs to be used as input to the computer programs two separate cases had to be considered.

Case a: $\bar{\xi}_{1} \neq 0, \bar{\xi}_{2} \neq 0, \bar{\xi}_{3}=0$

This resulted in coupling between the $\xi_{1}$ and $\xi_{2}$ modes and the input to equation (5.8) consisted of

$$
A_{o i} \cdot \cos i \bar{x} \rightarrow\left\{\begin{array}{l}
A_{k} \ell \cdot \cos k \bar{x} \cdot \cos \ell \bar{y} \\
B_{k} \ell \cdot \cos k \bar{x} \cdot \sin \ell \bar{y}
\end{array}\right.
$$


or

$$
C_{o i} \cdot \sin i \bar{x} \rightarrow\left\{\begin{array}{l}
C_{k} \cdot \sin k \bar{x} \cdot \cos l \bar{y} \\
D_{k} l \cdot \sin k \bar{x} \cdot \sin l \bar{y}
\end{array}\right.
$$

Case b: $\bar{\xi}_{1} \neq 0, \bar{\xi}_{2}=0, \bar{\xi}_{3} \neq 0$

This resulted in coupling between the $\xi_{1}$ and $\xi_{3}$ modes and the input to equation $(5,16)$ consisted of

$$
A_{o i} \cdot \cos i \bar{x} \rightarrow\left\{\begin{array}{l}
C_{k \ell} \cdot \sin k \bar{x} \cdot \cos \ell \bar{y} \\
D_{k \ell} \cdot \sin k \bar{x} \cdot \sin \ell \bar{y}
\end{array}\right.
$$

of

$$
c_{o \dot{1}} \cdot \sin i \bar{x} \rightarrow\left\{\begin{array}{l}
A_{k \ell} \cdot \cos k \bar{x} \cdot \cos \ell \bar{y} \\
B_{k \ell} \cdot \cos k \bar{x} \cdot \sin \ell \bar{y}
\end{array}\right.
$$

It should be mentioned here that the location of the origin in the circumferential direction was arbitrary. Thus either sine or cosine $\bar{y}$-dependence was admissible.

It was found that for a positive axisymmetric imperfection coupling in the $\xi_{3}$ mode would yield a lower value for $\lambda_{M}$ then coupling in the $\xi_{2}$ mode if both $\bar{\xi}_{2}$ and $\bar{\xi}_{3}$ were of the same size. Similarly for a negative axisymmetric imperfection coupling in the $\xi_{2}$ mode would result in a lower value for $\lambda_{M}$. 
A list of the numerical results for the shells tested is given in Table VII. The comparison between the results of this simplified analytical approach and the experimental values is shown in $\mathrm{T}$ able VIII. 


\section{CONCLUSION}

Due to the small number of shells tested the results obtained thus far must be considered only preliminary. However the following conclusions seemed to be warranted:

1. The initial imperfections of the shells surveyed so far were characterized by being composed predominantly of lower order modes (i.e. few circumferential and even fewer axial waves). The amplitudes of the higher order modes were in general very small (i.e. of the order of one per cent of the wall thickness or less).

2. As can be seen from the three-dimensional plots representing the growth of the prebuckling deformations just prior to buckling (Figs. 28 through 31 ) there was a very pronounced growth of imperfection components with long axial wave length and short circumferential wave length for all the shells tested. The number of circumferential waves of these dominant components was approximately equal to the number of circumferential waves in the postbuckled shape. The half wave length of the dominant components was equal to the length of the shell in the axial direction. However the axial half wave length of the postbuckled shape was much shorter than the axial half wave length of the dominant components in the prebuckling deformation.

3. There seemed to exist several "critical modal components" for every shell tested, all showing the same exponential growth close to the critical load instead of an isolated "critical modal component". In other words the mode of prebuckling 
deformation which apparently contributed to the reduction in buckling load of the shells had many Fourier coefficients. Furthermore, some of these components had relatively small initial values. That is, the "critical modal components" were not necessarily predominant in the initial imperfection shape.

4. The failure modes of shell A.7 with local buckling in two isolated waves and of shell A10 with one isolated wave at the upper edge seemed to support the claim expressed in reference 15 that local bucklings were caused by some pronounced localized initial imperfections of the test specimens. Upon comparing the local buckling pattern of shell A7 (Fig. 27) with the initial imperfection survey of the same shell (Fig. 19) it was strikingly evident that not only did the initial local buckling occur at the exact location of very pronounced localized initial defects of the test specimen but the same localized defects showed the most pronounced growth rate as can be seen from Fig. 26 just prior to the occurrence of local buckling. Similarly shell Al0 also had a very pronounced initial imperfection at the location of the local buckling as can be seen by comparing Fig. 32 showing the local buckling deformation with Fig. 22 showing the initial imperfection survey.

5. The comparison of the analytical results with the experimental values showed good agreement for the cases of global buckling. Apparently the dominant part of the measured imperfection surfaces was adequately approximated by the two terms of the pair of critical modal components. 
6. All the pairs of critical modal components were composed of an axisymmetric imperfection with one full wave in the axial direction and an asymmetric imperfection with one half wave in the axial direction. This seemed to confirm the conclusions of the visual observation of the growth of the prebuckling deformations where the most degrading imperfections, the ones with the most pronounced growth rate, were of long axial wave length. 
REFERENCES

1. Weingarten, V. I.; Morgan, E.T.; and Seide, P.: Final

Report on Development of Design Criteria for Elastic Stability of Thin Shell Structures. STL/TR-60-00019425, Space Technology Laboratories, 1960.

2. Stein, M.: The Effect on the Buckling of Perfect Cylinders of Prebuckling Deformations and Stresses Induced by Edge Support. Collected Papers on Instability of Shell Structures, NASA TN D-1510, 1962, pp. 217-227.

3. Fischer, E.: Uber den Einfluss der Gelenkigen Lagerung auf die Stabilitat Dunnwandiger Kreiszylinderschalen unter Axiallast und Innendruck. Z. Flugwissenschaften, Vol. 11, 1963, pp. $111-119$.

4. Almroth, B. O.: Influence of Edge Conditions on the Stability of Axially Compresed Cylindrical Shells. NASA CR-161, Feb. 1963.

5. Hoff, N. J.: The Effect of Edge Conditions on the Buckling of Thin Walled Circular Shells in Axial Compression. Proc. 11 th Int. Congress of Appl. Mech., Julius Springer Verlag, Berlin, 1964.

6. Kobayashi, S.: The Influence of the Boundary Conditions on the Buckling Load of Cylindrical Shells under Axial Compression. GALCIT SM 66-3, March 1966. 
7. Donnell, L. M.: A New Theory for the Buckling of Thin Cylinders under Axial Compression and Bending. Trans. Am. Soc. Mech. Eng., Vol. 56, 1934, p. 795.

8. Donnell, L. M. and Wan, C. C. : Effect of Imperfections on Buckling of Thin Cylinders and Columns under Axial Compression. Journ. Appl. Mech., Vol. 17, 1950, p. 73.

9. Koiter, W. T.: On the Stability of Elastic Equilibrium. Ph. D. Thesis, Delft, H. T. Paris, Amsterdam, 1945.

10. Koiter, W. T.: The Effect of Axisymmetric Imperfections on the Buckling of Cylindrical Shells under Axial Compression. Lockheed Missiles and Space Company, 6-90-63-86, Sunnyvale, California, Aug. 1963.

11. Koiter, W. T.: Elastic Stability and Postbuckling Behavior. Proc. Symposium Nonlinear Problems, (R. E. Langer ed.) University of Wisconsin Press, Madison, Wisconsin, 1963.

12. Hutchinson, J.: Axial Buckling of Pressurized Imperfect Cylindrical Shells, AIAA J.,Vol. 3, Aug. 1965, pp. $1461-1466$.

13. Thurston, E. A. and Freeland, M. A.: Buckling of Imperfect Cylinders under Axial Compression, NASA CR-541, July 1966. 
14. Babcock, C. D.: The Buckling of Cylindrical Shells with an Initial Imperfection under Axial Compression Loading. Ph. D. Thesis, California Institute of Technology, 1962.

15. Arbocz, J.: Buckling of Conical Shells under Axial Compression. GALCIT SM 68-6, Feb. 1968. 


\section{APPENDIX A}

The approximate solution of Donnell's equations for an imperfect cylindrical shell

$$
\begin{aligned}
& \frac{1}{E \mathrm{Et}} \nabla^{4} \mathrm{~F}-\frac{1}{\mathrm{R}} \mathrm{W},{ }_{\mathrm{xx}}+\frac{1}{2} \mathrm{~L}(\mathrm{~W}, \mathrm{~W}+2 \overline{\mathrm{W}})=0 \\
& \frac{E t^{3}}{12\left(1-v^{2}\right)} \nabla^{4} W+\frac{1}{R} F, x x-L(F, W+\bar{W})=0
\end{aligned}
$$

where the nonlinear operator $L$ is defined by

$$
L(S, T)=S,{ }_{x x} T,{ }_{y y}-2 S,{ }_{x y}{ }^{T}{ }_{x y}+S,{ }_{y y}{ }^{T}{ }_{x x}
$$

assumes that the initial imperfection shape is represented by

$$
\bar{W}=\bar{\xi}_{1} t \cos i \bar{x}+\xi_{2} t \cos k \bar{x} \cdot \cos \ell \bar{y}+\xi_{3} t \operatorname{sink} \bar{x} \cdot \cos \ell \bar{y}
$$

The equilibrium state of the axially loaded cylinder is approximated as:

$$
\begin{aligned}
& W=\frac{\nu}{E} \sigma t+w \\
& F=-\frac{1}{2} \sigma t y^{2}+f
\end{aligned}
$$

where the terms added to $w$ and $f$ constitute the prebuckling membrane solution for the perfect shell. Further $w$ is assumed as:

$$
w=\xi_{1} t \cos i \bar{x}+\xi_{2} t \cos k \bar{x} \cdot \cos \ell \bar{y}+\xi_{3} t \sin k \bar{x} \cdot \cos l \bar{y}
$$

Substituting the assumed form of $W$ and $\bar{W}$ into the compatibility 
equation (1) yields

$\frac{1}{\mathrm{Et}}\left(F,{ }_{x x x x}+2 F,_{x x y y}+F,_{y y y y}\right)=K_{1} \cos 2 k \bar{x}+K_{2} \cos i \bar{x}+K_{3} \cos 2 \ell \bar{y}$

$$
\begin{aligned}
& +K_{4} \sin 2 k \bar{x}+K_{5} \cos k \bar{x} \cdot \cos \ell \bar{y} \\
& +K_{6} \operatorname{sink} \bar{x} \cdot \cos \ell \bar{y}+K_{7} \cos (i+k) \bar{x} \cdot \cos \ell \bar{y} \\
& +K_{8} \cos (i-k) \bar{x} \cdot \cos \ell \bar{y}+K_{9} \sin (i+k) \bar{x} \cdot \cos \ell \bar{y} \\
& +K_{10} \sin (i-k) \bar{x} \cdot \cos \ell \bar{y}
\end{aligned}
$$

where

$$
\begin{aligned}
& \mathrm{K}_{1}=-\left(\frac{2 \pi}{\mathrm{L}}\right)^{2}\left(\frac{\mathrm{t}}{\mathrm{R}}\right)^{2} \mathrm{k}^{2} \ell^{2}\left[\xi_{2} \xi_{2}-\xi_{3} \xi_{3}+\frac{1}{2}\left(\xi_{2}^{2}-\xi_{3}^{2}\right)\right] \\
& \mathrm{K}_{2}=-\left(\frac{2 \pi}{\mathrm{L}}\right)^{2} \frac{\mathrm{t}}{\mathrm{R}} i^{2} \xi_{1} \\
& \mathrm{~K}_{3}=-\left(\frac{2 \pi}{\mathrm{L}}\right)^{2}\left(\frac{\mathrm{t}}{\mathrm{R}}\right)^{2} \mathrm{k}^{2} \ell^{2}\left[\xi_{2} \xi_{2}+\xi_{3} \xi_{3}+\frac{1}{2}\left(\xi_{2}^{2}+\xi_{3}^{2}\right)\right] \\
& \mathrm{K}_{4}=-\left(\frac{2 \pi}{\mathrm{L}}\right)^{2}\left(\frac{\mathrm{t}}{\mathrm{R}}\right)^{2} \mathrm{k}^{2} \ell^{2}\left[\xi_{3} \xi_{2}+\left(\xi_{2}+\xi_{2}\right) \xi_{3}\right] \\
& \mathrm{K}_{5}=-\left(\frac{2 \pi}{\mathrm{L}}\right)^{2} \frac{\mathrm{t}}{\mathrm{R}} \mathrm{k}^{2} \xi_{2} \\
& \mathrm{~K}_{6}=-\left(\frac{2 \pi}{\mathrm{L}}\right)^{2} \frac{\mathrm{t}}{\mathrm{R}} \mathrm{k}^{2} \xi_{3} \\
& \mathrm{~K}_{7}=-\left(\frac{2 \pi}{\mathrm{L}}\right)^{2}\left(\frac{\mathrm{t}}{\mathrm{R}}\right)^{2} \frac{\mathrm{i}^{2} \ell^{2}}{2}\left[\xi_{2} \xi_{1}+\left(\xi_{1}+\xi_{1}\right) \xi_{2}\right] \\
& \mathrm{K}_{8}=-\left(\frac{2 \pi}{\mathrm{L}}\right)^{2}\left(\frac{\mathrm{t}}{\mathrm{R}}\right)^{2} \frac{\mathrm{i}^{2} \ell^{2}}{2}\left[\xi_{2} \xi_{1}+\left(\xi_{1}+\xi_{1}\right) \xi_{2}\right] \\
& \mathrm{K}_{9}=-\left(\frac{2 \pi}{\mathrm{L}}\right)^{2}\left(\frac{\mathrm{t}}{\mathrm{R}}\right)^{2} \frac{\mathrm{i}^{2} \ell^{2}}{2}\left[\xi_{3} \xi_{1}+\left(\xi_{1}+\xi_{1}\right) \xi_{3}\right] \\
& \mathrm{K}_{10}=\left(\frac{2 \pi}{\mathrm{L}}\right)^{2}\left(\frac{\mathrm{t}}{\mathrm{R}}\right)^{2} \frac{i^{2} \ell^{2}}{2}\left[\xi_{3} \xi_{1}+\left(\xi_{1}+\xi_{1}\right) \xi_{3}\right]
\end{aligned}
$$


The boundary conditions of the finite shell will be neglected, therefore only a particular solution of equation (8) is needed. To obtain such a particular solution let

$$
F=-\frac{1}{2} \sigma t y^{2}+f_{p}
$$

where

$$
\begin{aligned}
f_{p}= & A \cos 2 k \bar{x}+B \operatorname{cosi} \bar{x}+C \cos 2 \ell \bar{y}+D \sin 2 k \bar{x}+\widetilde{E} \cos k \bar{x} \cdot \cos \ell \bar{y} \\
& +F \sin k \bar{x} \cdot \cos \ell \bar{y}+C \cos (i+k) \bar{x} \cdot \cos \ell \bar{y}+H \cos (i-k) \bar{x} \cdot \cos \ell \bar{y} \\
& +I \sin (i+k) \bar{x} \cdot \cos \ell \bar{y}+J \sin (i-k) \bar{x} \cdot \cos \ell \bar{y}
\end{aligned}
$$

Substituting this expression into equation (2) and equating coefficients of like terms yields:

$$
\begin{aligned}
& A=\operatorname{Et}\left(\frac{L}{2 \pi}\right)^{4} \frac{1}{(2 k)^{4}} K_{1}=-\operatorname{Et}\left(\frac{L}{2 \pi}\right)^{2}\left(\frac{t}{R}\right)^{2} \frac{\ell^{2}}{16 k^{2}}\left[\xi_{2} \xi_{2}-\xi_{3} \xi_{3}+\frac{1}{2}\left(\xi_{2}^{2}-\xi_{3}^{2}\right)\right] \\
& B=\operatorname{Et}\left(\frac{L}{2 \pi}\right)^{4} \frac{1}{i^{4}} K_{2}=-\operatorname{Et}\left(\frac{L}{2 \pi}\right)^{2} \frac{t}{R} \frac{1}{i^{2}} \xi_{1} \\
& C=\operatorname{EtR}^{4} \frac{1}{(2 \ell)^{4}} K_{3}=-\operatorname{EtR}^{4}\left(\frac{2 \pi}{L}\right)^{2}\left(\frac{t}{R}\right)^{2} \frac{k^{2}}{16 \ell^{2}}\left[\xi_{2} \xi_{2}+\xi_{3} \xi_{3}+\frac{1}{2}\left(\xi_{2}^{2}+\xi_{3}^{2}\right)\right] \\
& D=\operatorname{Et}\left(\frac{L}{2 \pi}\right)^{4} \frac{1}{(2 k)^{4}} K_{4}=-\operatorname{Et}\left(\frac{L}{2 \pi}\right)^{2}\left(\frac{t}{R}\right)^{2} \frac{\ell^{2}}{16 k^{2}}\left[\xi_{3} \xi_{2}+\left(\xi_{2}+\xi_{2}\right) \xi_{3}\right] \\
& \widetilde{E}=\operatorname{Et}\left[\left(\frac{2 \pi}{L}\right)^{2} k^{2}+\frac{\ell^{2}}{R^{2}}\right]^{-2} K_{5}=-\operatorname{Et}\left(\frac{2 \pi}{L}\right)^{2} \frac{t}{R} k^{2}\left[\left(\frac{2 \pi}{L}\right)^{2} k^{2}+\frac{\ell^{2}}{R^{2}}\right]^{-2} \xi_{2} \\
& F=E t\left[\left(\frac{2 \pi}{L}\right)^{2} k^{2}+\frac{\ell^{2}}{R^{2}}\right]^{-2} K_{6}=-E t\left(\frac{2 \pi}{L}\right)^{2} \frac{t}{R} k^{2}\left[\left(\frac{2 \pi}{L}\right)^{2} k^{2}+\frac{l^{2}}{R^{2}}\right]^{-2} \xi_{3}
\end{aligned}
$$




$$
\begin{aligned}
G= & E t\left[\left(\frac{2 \pi}{L}\right)^{2}(i+k)^{2}+\frac{l^{2}}{R^{2}}\right]^{-2} K_{7} \\
& =-E t\left(\frac{2 \pi}{L}\right)^{2}\left(\frac{t}{R}\right)^{2} \frac{i^{2} l^{2}}{2}\left[\left(\frac{2 \pi}{L}\right)^{2}(i+k)^{2}+\frac{l^{2}}{R^{2}}\right]^{-2}\left[\xi_{2} \xi_{1}+\left(\xi_{1}+\xi_{1}\right) \xi_{2}\right] \\
H= & E t\left[\left(\frac{2 \pi}{L}\right)^{2}(i-k)^{2}+\frac{l^{2}}{R^{2}}\right]^{-2} K_{8} \\
& =-E t\left(\frac{2 \pi}{L}\right)^{2}\left(\frac{t}{R}\right)^{2} \frac{i^{2} l^{2}}{2}\left[\left(\frac{2 \pi}{L}\right)^{2}(i-k)^{2}+\frac{l^{2}}{R^{2}}\right]^{-2}\left[\xi_{2} \xi_{1}+\left(\xi_{1}+\xi_{1}\right) \xi_{2}\right] \\
I= & E t\left[\left(\frac{2 \pi}{L}\right)^{2}(i+k)^{2}+\frac{l^{2}}{R^{2}}\right]^{-2} K_{9} \\
& =-E t\left(\frac{2 \pi}{L}\right)^{2}\left(\frac{t}{R}\right)^{2} \frac{i^{2} l^{2}}{2}\left[\left(\frac{2 \pi}{L}\right)^{2}(i+k)^{2}+\frac{l^{2}}{R^{2}}\right]^{-2}\left[\xi_{3} \xi_{1}+\left(\xi_{1}+\xi_{1}\right) \xi_{3}\right] \\
J= & E t\left[\left(\frac{2 \pi}{L}\right)^{2}(i-k)^{2}+\frac{\ell^{2}}{R^{2}}\right]^{-2} K_{10} \\
& =E t\left(\frac{2 \pi}{L}\right)^{2}\left(\frac{t}{R}\right)^{2} \frac{i^{2} l^{2}}{2}\left[\left(\frac{2 \pi}{L}\right)^{2}(i-k)^{2}+\frac{l^{2}}{R^{2}}\right]^{-2}\left[\xi_{3} \xi_{1}+\left(\xi_{1}+\xi_{1}\right) \xi_{3}\right]
\end{aligned}
$$

Substituting the assumed form for $W$ and $\bar{W}$ and the computed particular solution for $F$ as given by equations (4), (7) and (10) into the equilibrium equation (2) yields, after multiplying out, regrouping and simplifying through the use of trigonometric identities the following expression for the ERROR $\varepsilon_{\mathrm{N}}$ :

$$
\begin{aligned}
& \left\{\frac{E t^{4}}{\left(12\left(1-\nu^{2}\right)\right.}\left(\frac{2 \pi}{L}\right)^{2} i^{4} \xi_{1}-\frac{i^{2}}{R} B-t^{2} i^{2}\left(\xi_{1}+\xi_{1}\right) \sigma\right. \\
& \left.-\frac{t}{4 R^{2}} i^{2} \ell^{2}\left[\left(\xi_{2}+\xi_{2}\right)(G+H)+\left(\xi_{3}+\xi_{3}\right)(I-J)\right]\right\} \cdot \cos i \bar{x}
\end{aligned}
$$




$$
\begin{aligned}
& +\left\{\frac{E t^{4}}{12\left(1-\nu^{2}\right)}\left[\left(\frac{2 \pi}{L}\right)^{2} k^{2}+\left(\frac{l}{R}\right)^{2}\right]^{2}\left(\frac{L}{2 \pi}\right)^{2} \xi_{2}-\frac{k^{2}}{R} \widetilde{E}-t^{2} k^{2}\left(\xi_{2}+\xi_{2}\right) \sigma\right. \\
& -\frac{t \ell^{2}}{2 \mathrm{R}^{2}}\left[4 \mathrm{k}^{2}\left[\left(\xi_{2}+\bar{\xi}_{2}\right)(\mathrm{A}+\mathrm{C})+\left(\xi_{3}+\bar{\xi}_{3}\right) \mathrm{D}\right]\right. \\
& \left.\left.+i^{2}\left(\xi_{1}+\bar{\xi}_{1}\right)(\mathrm{G}+\mathrm{H})\right]\right\} \cos \mathrm{k} \overline{\mathrm{x}} \cdot \cos \ell \overline{\mathrm{y}} \\
& +\left\{\frac{E t^{4}}{12\left(1-\nu^{2}\right)}\left[\left(\frac{2 \pi}{L}\right)^{2} k^{2}+\left(\frac{l}{R}\right)^{2}\right]^{2}\left(\frac{L}{2 \pi}\right)^{2} \xi_{3}-\frac{k^{2}}{R} F-t^{2} k^{2}\left(\xi_{3}+\xi_{3}\right) \sigma\right. \\
& -\frac{t}{2 \mathrm{R}^{2}} \ell^{2}\left[4 \mathrm{k}^{2}\left[\left(\xi_{2}+\bar{\xi}_{2}\right) \mathrm{D}+\left(\xi_{3}+\bar{\xi}_{3}\right)(\mathrm{C}-\mathrm{A})\right]\right. \\
& \left.\left.+i^{2}\left(\xi_{1}+\bar{\xi}_{1}\right)(I-J)\right]\right\} \operatorname{sink} \bar{x} \cdot \cos \ell \bar{y} \\
& +\left\{\frac{-4 \mathrm{k}^{2}}{\mathrm{R}} \mathrm{A}-\frac{\mathrm{t}}{\mathrm{R}^{2}} \mathrm{k}^{2} \ell^{2}\left[\left(\xi_{2}+\bar{\xi}_{2}\right) \widetilde{\mathrm{E}}-\left(\xi_{3}+\bar{\xi}_{3}\right) \mathrm{F}\right]\right\} \cos 2 \mathrm{k} \overline{\mathrm{x}} \\
& +\left\{\frac{-(i-k)^{2}}{R} H-\frac{t}{2 R^{2}} i^{2} \ell^{2}\left[\left(\xi_{1}+\xi_{1}\right) \widetilde{E}+\left(\xi_{2}+\xi_{2}\right) B\right]\right\} \cos (i-k) \bar{x} \cdot \cos \ell \bar{y} \\
& +\left\{\frac{-(i-k)^{2}}{R} J-\frac{t}{2 R^{2}} i^{2} \ell^{2}\left[\left(\xi_{1}+\bar{\xi}_{1}\right) F+\left(\xi_{2}+\xi_{2}\right) B\right]\right\} \sin (i-k) \bar{x} \cdot \cos \ell \bar{y} \\
& +\left\{\frac{-t}{4 \mathrm{R}^{2}} i^{2} \ell^{2}\left[\left(\xi_{3}+\xi_{3}\right)(\mathrm{H}-\mathrm{G})+\left(\xi_{2}+\xi_{2}\right)(\mathrm{I}+\mathrm{J})\right]\right\} \operatorname{sini\overline {x}} \\
& +\left\{\frac{-4 k^{2}}{R} D-\frac{t}{R^{2}} k^{2} \ell^{2}\left[\left(\xi_{2}+\bar{\xi}_{2}\right) F+\left(\xi_{3}+\bar{\xi}_{3}\right) \widetilde{E}\right]\right\} \sin 2 k \bar{x} \\
& +\left\{\frac{-t}{R^{2}} k^{2} \ell^{2}\left[\left(\xi_{2}+\xi_{2}\right) \widetilde{E}+\left(\xi_{3}+\xi_{3}\right) F\right]\right\} \cos 2 \ell \bar{y}
\end{aligned}
$$


$+\left\{\frac{-t}{4 \mathrm{R}^{2}} \ell^{2}\left[16 \mathrm{i}^{2}\left(\xi_{1}+\xi_{1}\right) \mathrm{C}+(2 \mathrm{k}+\mathrm{i})^{2}\left[\left(\xi_{2}+\bar{\xi}_{2}\right) \mathrm{G}+\left(\xi_{3}+\xi_{3}\right) \mathrm{I}\right]\right.\right.$ $\left.\left.+(2 k-i)^{2}\left[\left(\xi_{2}+\xi_{2}\right) H-\left(\xi_{3}+\xi_{3}\right) J\right]\right]\right\} \cos i \bar{x} \cdot \cos 2 \ell \bar{y}$

$+\left\{\frac{-t}{4 \mathrm{R}^{2}} l^{2}(2 \mathrm{k}+\mathrm{i})^{2}\left[\left(\xi_{2}+\bar{\xi}_{2}\right) \mathrm{G}-\left(\xi_{3}+\bar{\xi}_{3}\right) \mathrm{I}\right]\right\} \cos (\mathrm{i}+2 \mathrm{k}) \overline{\mathrm{x}}$

$+\left\{\frac{-t}{4 R^{2}} \ell^{2}(2 k+i){ }^{2}\left[\left(\xi_{2}+\xi_{2}\right) I+\left(\xi_{3}+\xi_{3}\right) G\right]\right\} \sin (i+2 k) \bar{x}$

$+\left\{\frac{-t}{4 \mathrm{R}^{2}} \ell^{2}\left[(2 \mathrm{k}+\mathrm{i})^{2}\left[\left(\xi_{2}+\xi_{2}\right) I-\left(\xi_{3}+\xi_{3}\right) G\right]\right.\right.$

$\left.\left.+(2 k-i)^{2}\left[\left(\xi_{2}+\bar{\xi}_{2}\right)\right]+\left(\xi_{3}+\xi_{3}\right) H\right]\right\} \sin \bar{x} \cdot \cos 2 \ell \bar{y}$

$+\left\{\frac{-(i+k)^{2}}{R} G-\frac{t}{R^{2}} \frac{i^{2} l^{2}}{2}\left[\left(\xi_{1}+\xi_{1}\right) \widetilde{E}+\left(\xi_{3}+\bar{\xi}_{3}\right) B\right]\right\} \cos (i+k) \bar{x} \cdot \cos \ell \bar{y}$

$+\left\{\frac{-(i+k)^{2}}{R} I-\frac{t}{R^{2}} \frac{i^{2} l^{2}}{2}\left[\left(\xi_{1}+\xi_{1}\right) F+\left(\xi_{3}+\xi_{3}\right) B\right]\right\} \sin (i+k) \bar{x} \cdot \cos l \bar{y}$

$+\left\{\frac{-t}{\mathrm{R}^{2}} 2 \mathrm{k}^{2} \ell^{2}\left(\xi_{3}+\bar{\xi}_{3}\right) C\right\} \sin \mathrm{k} \overline{\mathrm{x}} \cdot \cos 3 \ell \overline{\mathrm{y}}$

$+\left\{\frac{-t}{R^{2}} 2 k^{2} \ell^{2}\left(\xi_{2}+\xi_{2}\right) C\right\} \cos k \bar{x} \cdot \cos 3 \ell \bar{y}$

$+\left\{\frac{-t}{4 R^{2}}(2 k-i)^{2} \ell^{2}\left[\left(\xi_{2}+\bar{\xi}_{2}\right) H+\left(\xi_{3}+\xi_{3}\right) J\right]\right\} \cos (2 k-i) \bar{x}$

$+\left\{\frac{-t}{4 R^{2}}(2 k-i)^{2} \ell^{2}\left[\left(\xi_{3}+\bar{\xi}_{3}\right) H-\left(\xi_{2}+\bar{\xi}_{2}\right) J\right]\right\} \sin (2 k-i) \bar{x}$

$+\left\{\frac{-t}{4 R^{2}} i^{2} \ell^{2}\left[\left(\xi_{2}+\bar{\xi}_{2}\right) G-\left(\xi_{3}+\bar{\xi}_{3}\right) I\right]\right\} \cos (2 k+i) \bar{x} \cdot \cos 2 \ell \bar{y}$ 


$$
\begin{aligned}
& +\left\{\frac{-t}{4 R^{2}} i^{2} \ell^{2}\left[\left(\xi_{2}+\bar{\xi}_{2}\right) I+\left(\xi_{3}+\xi_{3}\right) G\right]\right\} \sin (2 k+i) \bar{x} \cdot \cos 2 \ell \bar{y} \\
& +\left\{\frac{-t}{4 R^{2}} i^{2} \ell^{2}\left[\left(\xi_{2}+\xi_{2}\right) H+\left(\xi_{3}+\xi_{3}\right) J\right]\right\} \cos (2 k-i) \bar{x} \cdot \cos 2 \ell \bar{y} \\
& +\left\{\frac{-t}{4 R^{2}} i^{2} \ell^{2}\left[\left(\xi_{3}+\xi_{3}\right) H-\left(\xi_{2}+\xi_{2}\right) J\right]\right\} \sin (2 k-i) \bar{x} \cdot \cos 2 \ell \bar{y} \\
& +\left\{\frac{-t}{R^{2}} 2 k^{2} \ell^{2}\left[\left(\xi_{2}+\xi_{2}\right) A-\left(\xi_{3}+\xi_{3}\right) D\right]\right\} \cos 3 k \bar{x} \cdot \cos \ell \bar{y} \\
& +\left\{\frac{-t}{R^{2}} 2 k^{2} \ell^{2}\left[\left(\xi_{2}+\bar{\xi}_{2}\right) D+\left(\xi_{3}+\xi_{3}\right) A\right]\right\} \sin 3 k \bar{x} \cdot \cos \ell \bar{y} \\
& +\left\{\frac{-t}{2 R^{2}} i^{2} \ell^{2}\left(\xi_{1}+\xi_{1}\right) G\right\} \cos (2 i+k) \bar{x} \cdot \cos \ell \bar{y} \\
& +\left\{\frac{-t}{2 R^{2}} i^{2} \ell^{2}\left(\xi_{1}+\xi_{1}\right) H\right\} \cos (2 i-k) \bar{x} \cdot \cos \ell \bar{y} \\
& +\left\{\frac{-t}{2 R^{2}} i^{2} \ell^{2}\left(\xi_{1}+\bar{\xi}_{1}\right) I\right\} \sin (2 i+k) \bar{x} \cdot \cos \ell \bar{y} \\
& +\left\{\frac{-t}{2 R^{2}} i^{2} \ell^{2}\left(\xi_{1}+\xi_{1}\right) J\right\} \sin (2 i-k) \bar{x} \cdot \cos \ell \bar{y} \\
& =e_{N}\left(\xi_{1}, \xi_{2}, \xi_{3}, \bar{x}, \bar{y}\right)
\end{aligned}
$$

where the ERROR $\varepsilon_{N}$ is a function of the unknown amplitudes $\xi_{1}, \xi_{2}$ and $\xi_{3}$ of the assumed radial displacement $W$.

Using Galerkin's idea of minimization of the error with respect to a set of given functions leads to a system of three nonlinear algebraic equations in the three unknowns $\xi_{1}, \xi_{2}, \xi_{3}$. Here these 
equations will be obtained from the following integrals:

$$
\begin{aligned}
& \int_{0}^{2 \pi} \int_{0}^{2 \pi} \varepsilon_{N}\left(\xi_{1}, \xi_{2}, \xi_{3}, \bar{x}, \bar{y}\right) \cdot \cos i \bar{x} d \bar{x} d \bar{y}=0 \\
& \int_{0}^{2 \pi} \int_{0}^{2 \pi} \varepsilon_{N}\left(\xi_{1}, \xi_{2}, \xi_{3}, \bar{x}, \bar{y}\right) \cdot \cos k \bar{x} \cdot \cos \ell \bar{y} d \bar{x} d \bar{y}=0 \\
& \int_{0}^{2 \pi} \int_{0}^{2 \pi} \varepsilon_{N}\left(\xi_{1}, \xi_{2}, \xi_{3}, \bar{x}, \bar{y}\right) \cdot \sin k \bar{x} \cdot \cos l \bar{y} d \bar{x} d \bar{y}=0
\end{aligned}
$$

Imposing the restriction $k=i / 2$ and carrying out the indicated integrations leads to:

$$
\begin{aligned}
& \frac{E t^{4}}{12\left(1-v^{2}\right)}\left(\frac{2 \pi}{L}\right)^{2} i^{4} \xi_{1} \frac{-i^{2}}{R} B-t^{2} i^{2}\left(\xi_{1}+\xi_{1}\right) \sigma \\
& -\frac{t}{4 R^{2}} i^{2} l^{2}\left[\left(\xi_{2}+\xi_{2}\right)(G+H)+\left(\xi_{3}+\xi_{3}\right)(I-J)\right] \\
& \quad \frac{-4 k^{2}}{R} A-\frac{t}{R^{2}} k^{2} \ell^{2}\left[\left(\xi_{2}+\xi_{2}\right) \widetilde{E}-\left(\xi_{3}+\xi_{3}\right) F\right]=0 \\
& \frac{E t}{\left(12\left(1-v^{2}\right)\right.}\left(\frac{L}{2 \pi}\right)^{2}\left[\left(\frac{2 \pi}{L}\right)^{2} k^{2}+\left(\frac{\ell}{R}\right)^{2}\right]^{2} \xi_{2}-\frac{k^{2}}{R} \widetilde{E}^{4}-t^{2} k^{2}\left(\xi_{2}+\xi_{2}\right) \sigma \\
& -\frac{t}{2 R^{2}} \ell^{2}\left[4 k^{2}\left[\left(\xi_{2}+\xi_{2}\right)(A+C)+\left(\xi_{3}+\xi_{3}\right) D\right]\right. \\
& \left.+i^{2}\left(\xi_{1}+\xi_{1}\right)(G+H)\right]-\frac{(i-k)^{2}}{R} H-\frac{t}{2 R^{2}} i^{2} \ell^{2}\left[\left(\xi_{1}+\xi_{1}\right) \widetilde{E}+\left(\xi_{2}+\xi_{2}\right) B\right]=0
\end{aligned}
$$




$$
\begin{aligned}
& \frac{E t^{4}}{12\left(1-v^{2}\right)}\left(\frac{L}{2 \pi}\right)^{2}\left[\left(\frac{2 \pi}{L}\right)^{2} k^{2}+\left(\frac{l}{R}\right)^{2}\right]^{2} \xi_{3}-\frac{k^{2}}{R} F-t^{2} k^{2}\left(\xi_{3}+\xi_{3}\right) \sigma \\
& -\frac{t}{2 R^{2}} l^{2}\left[4 k^{2}\left[\left(\xi_{2}+\xi_{2}\right) D+\left(\xi_{3}+\xi_{3}\right)(C-A)\right]+i^{2}\left(\xi_{1}+\xi_{1}\right)(I-J)\right] \\
& -\frac{(i-k)^{2}}{R} J-\frac{t}{2 R^{2}} i^{2} \ell^{2}\left[\left(\xi_{1}+\xi_{1}\right) F+\left(\xi_{3}+\xi_{3}\right) B\right]=0
\end{aligned}
$$

Without the restriction $\mathrm{k}=\mathrm{i} / 2$ the underlined terms would vanish because of the orthogonality properties of the respective trigonometric functions and the resulting equations would contain only cubic nonlinearities in the unknown amplitudes $\xi_{1}, \xi_{2}$ and $\xi_{3}$. Substituting for the coefficients $A, B, \ldots$. I and $J$ from equations (11) and introducing the nondimensional parameters defined in equations (2.11) yields the NONLINEAR BUCKLING EQUATIONS (2.12) - (2.14). 


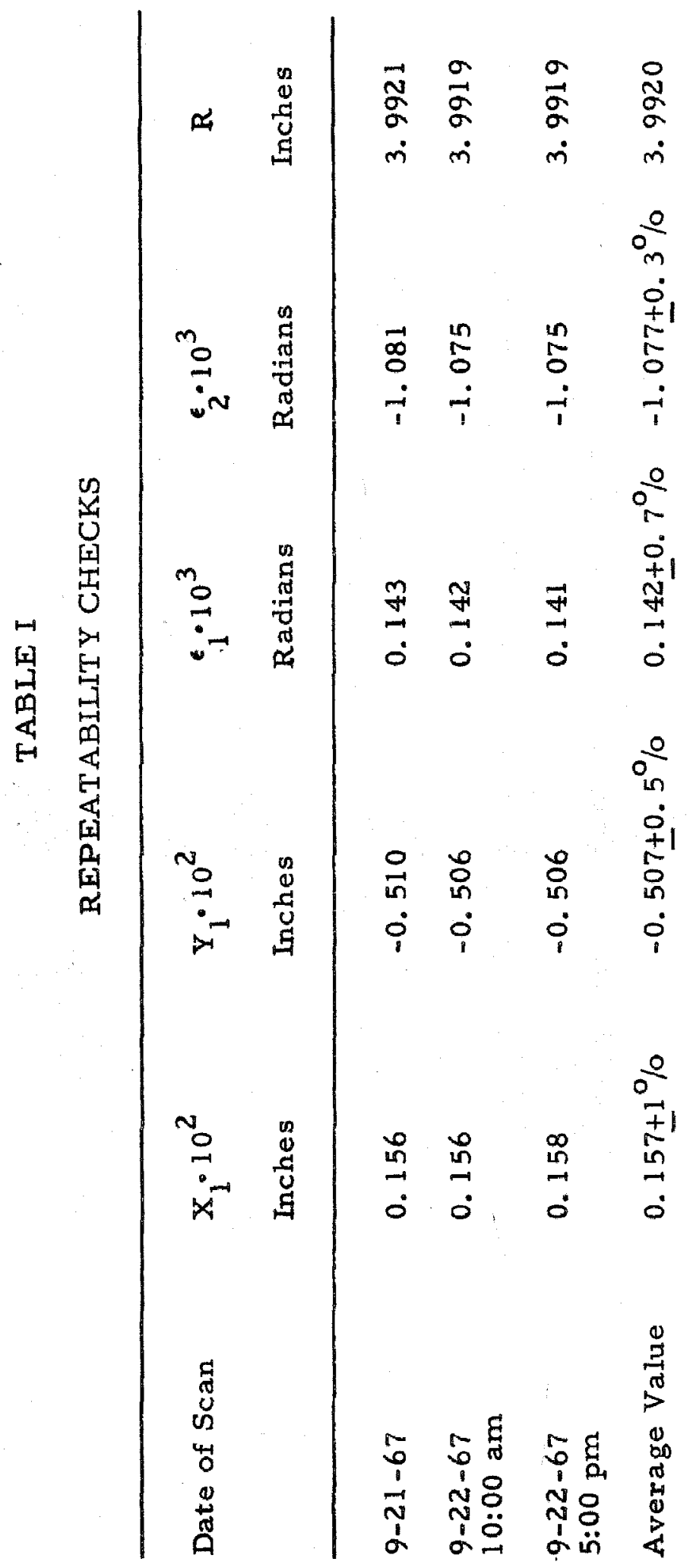




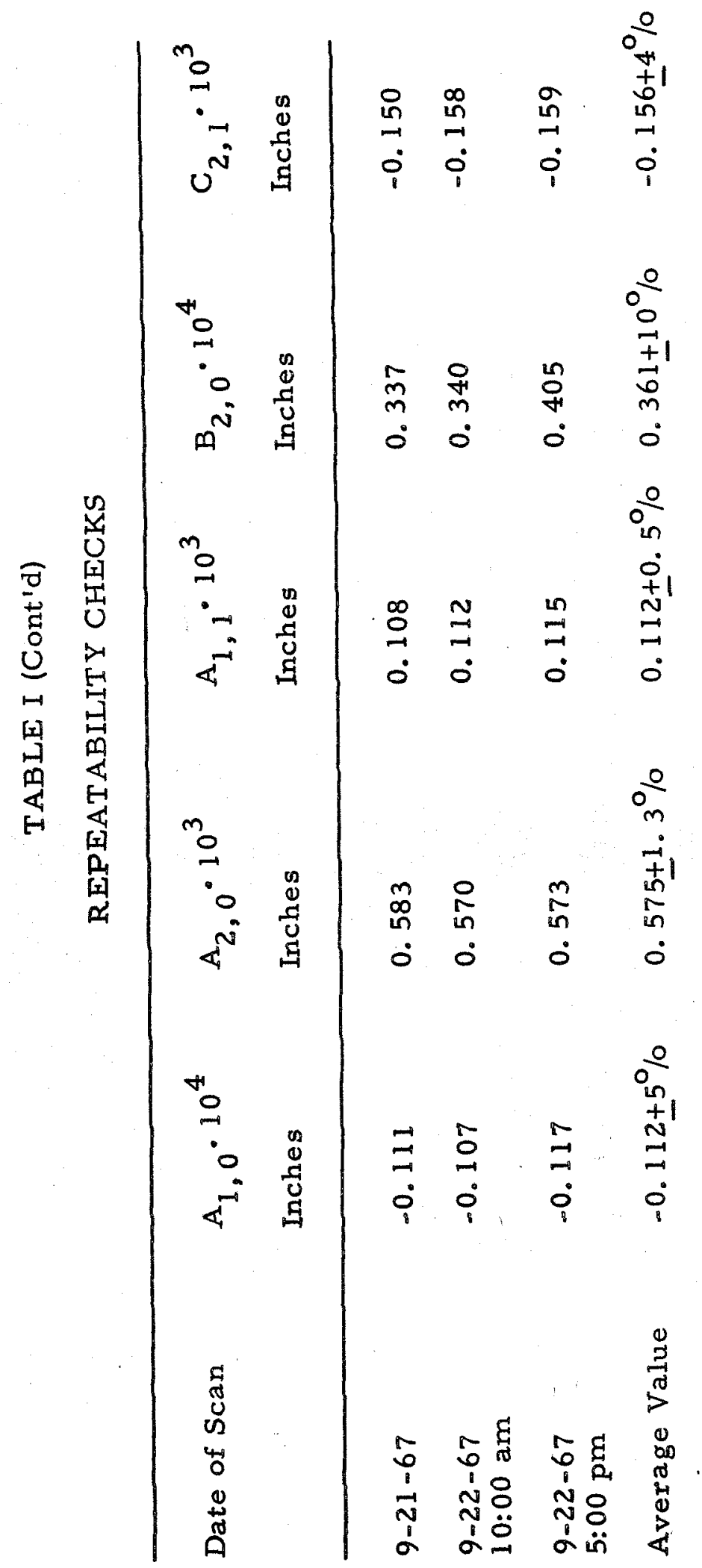




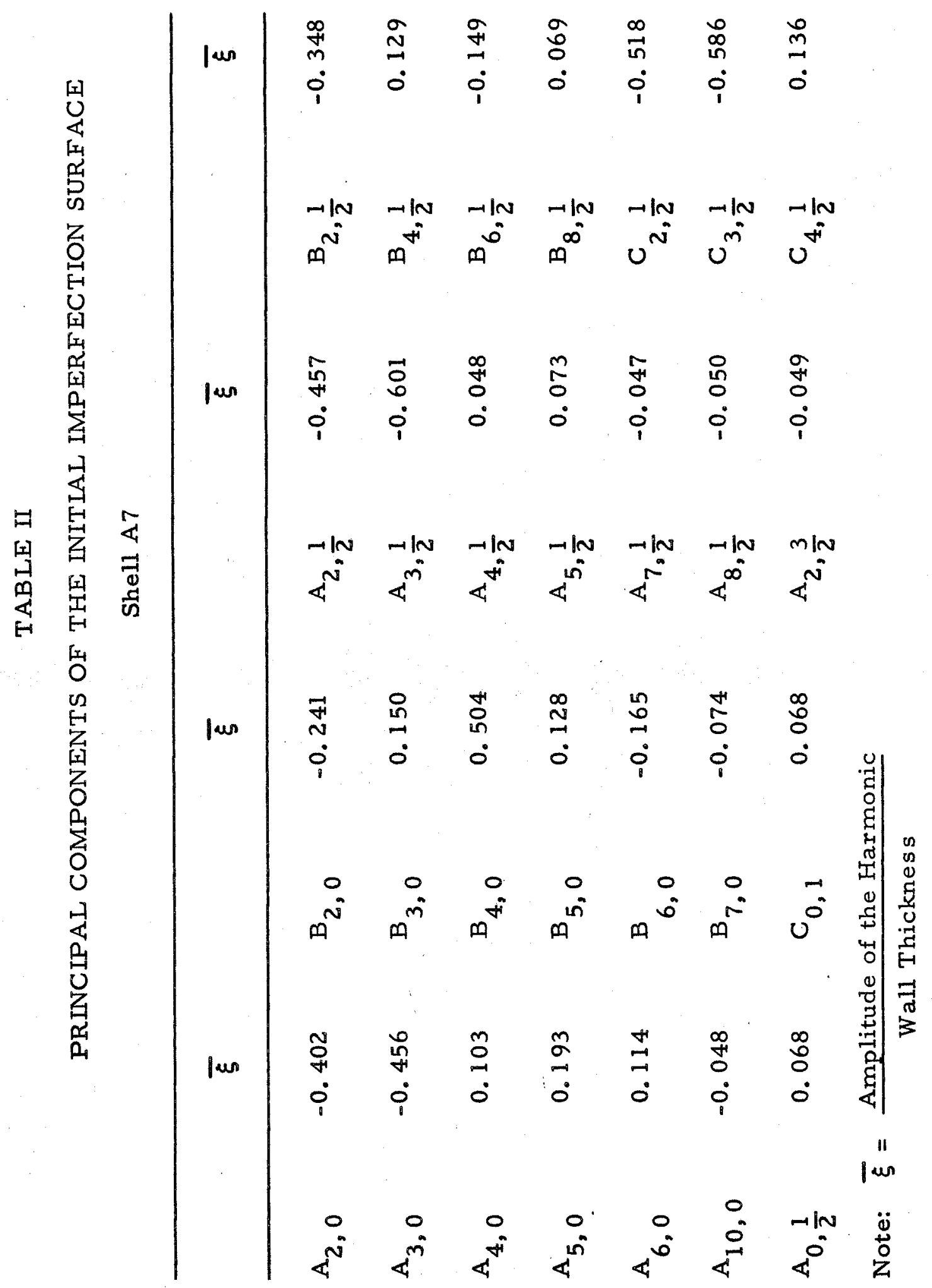




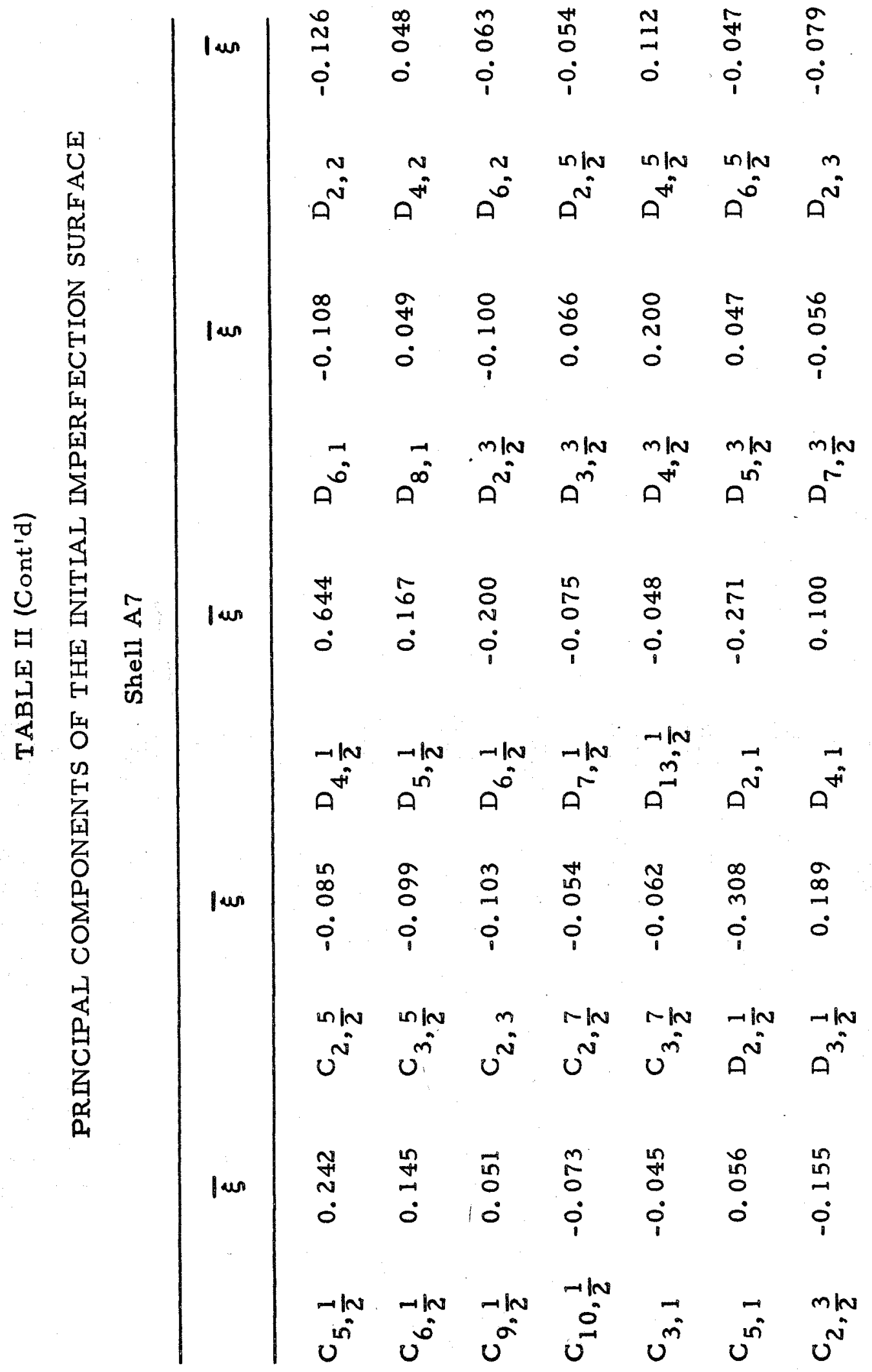




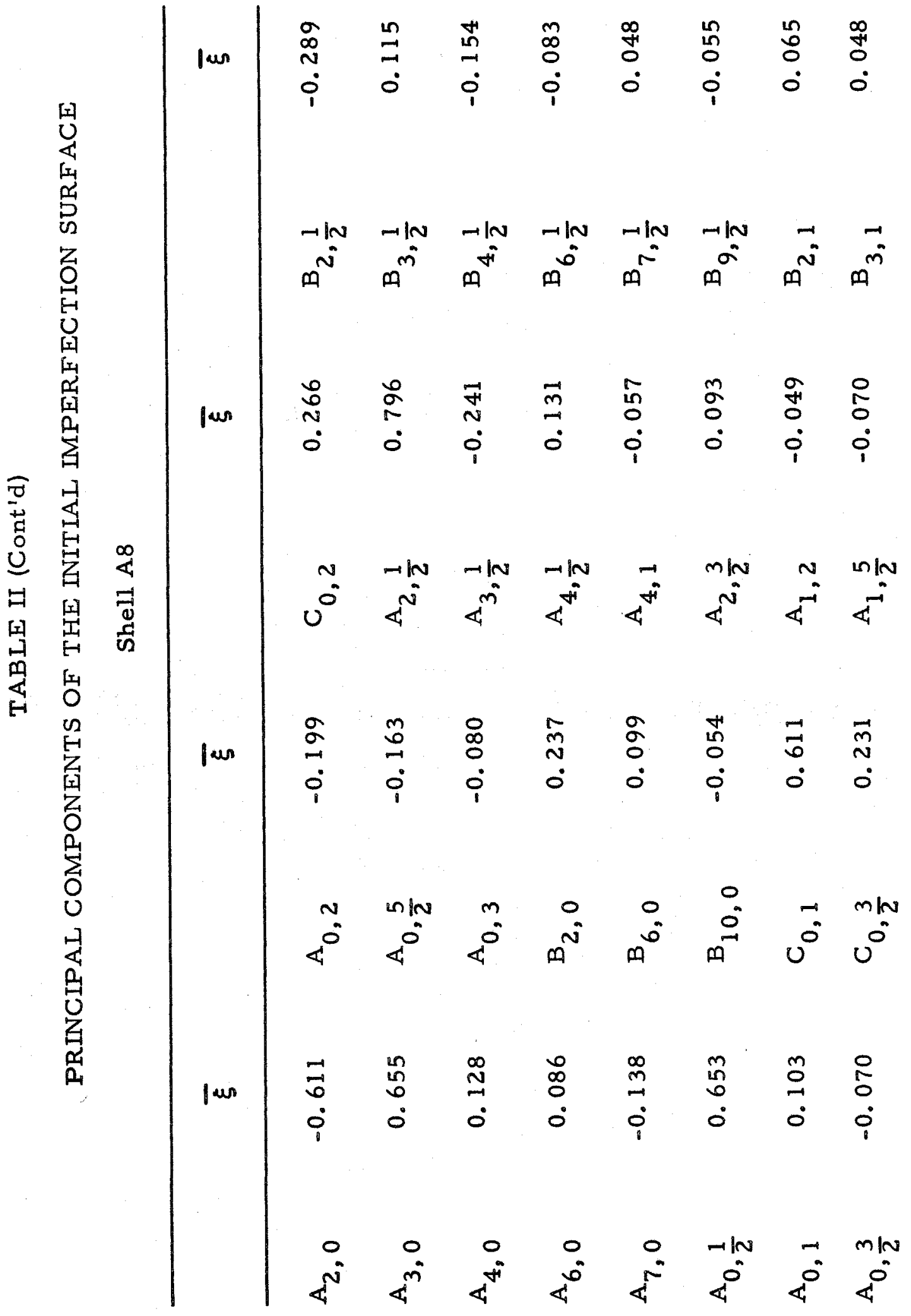




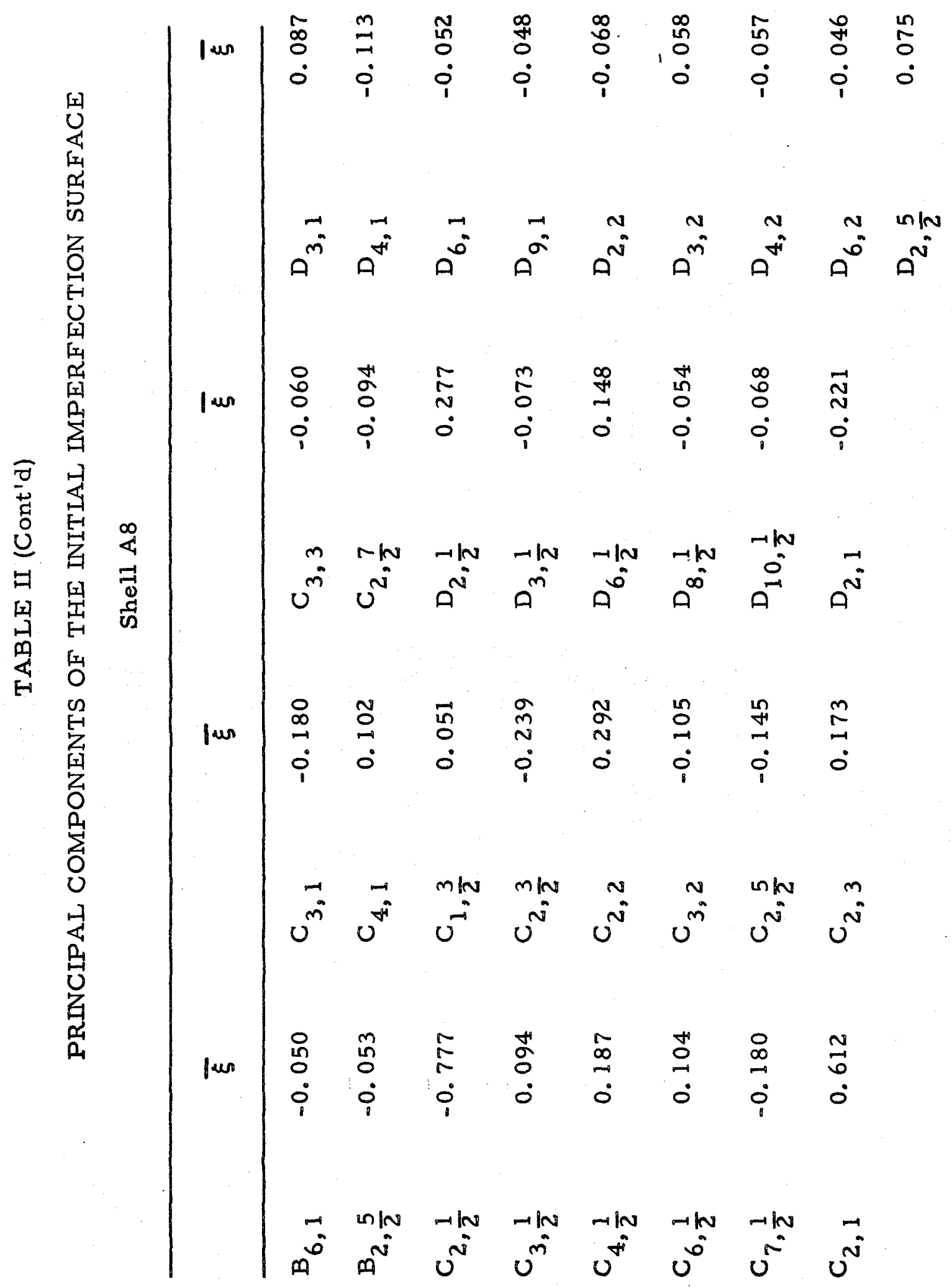




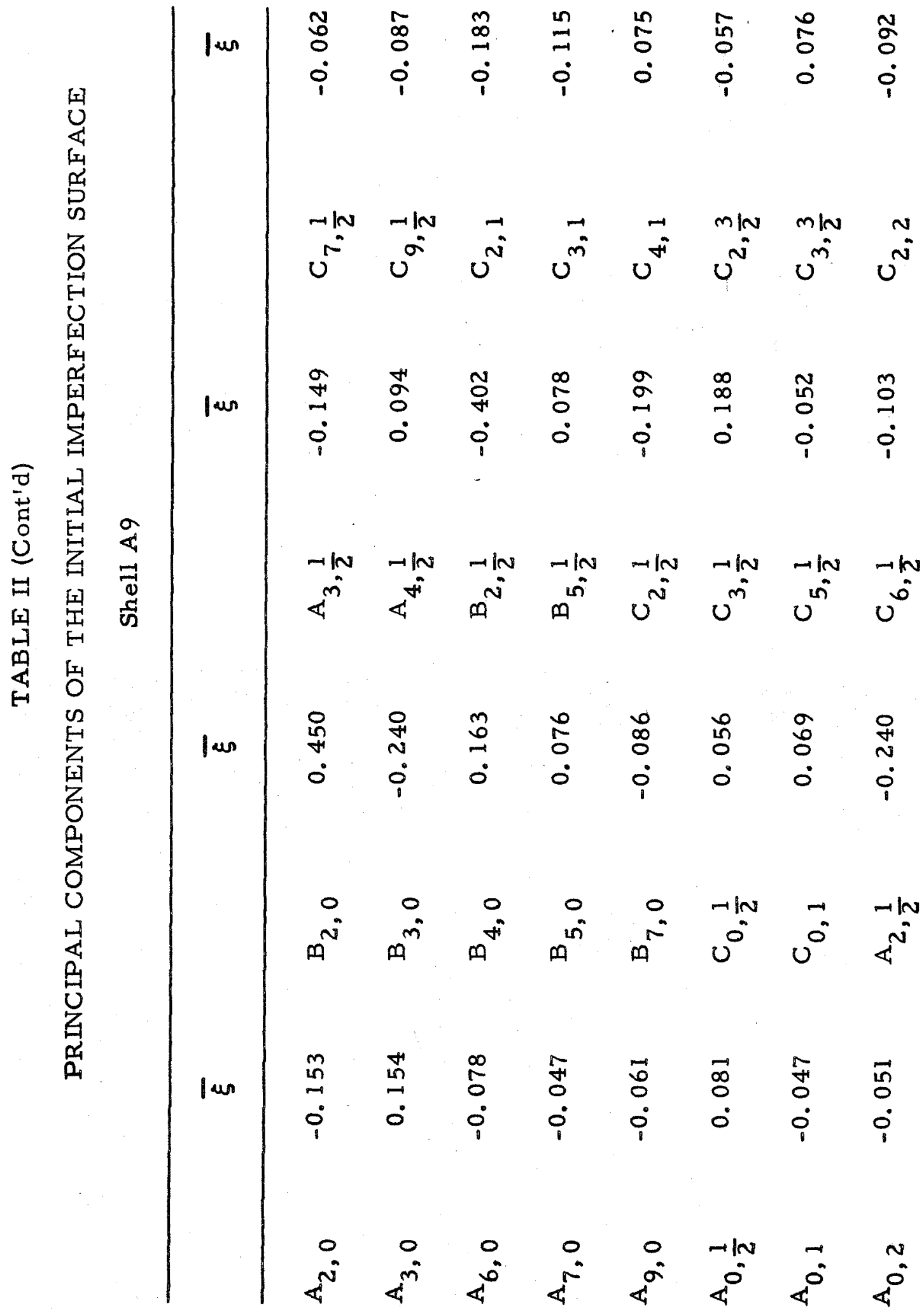




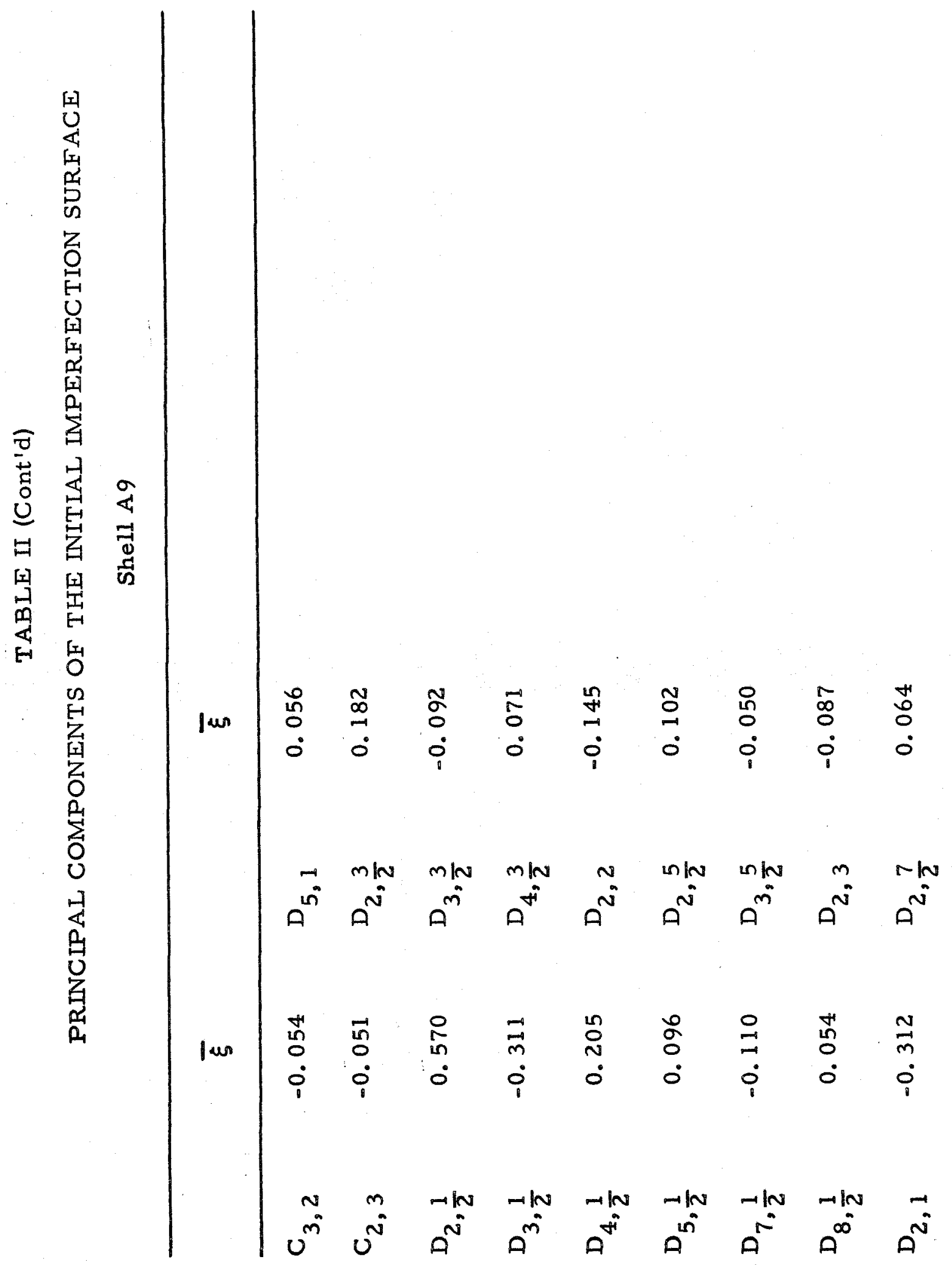




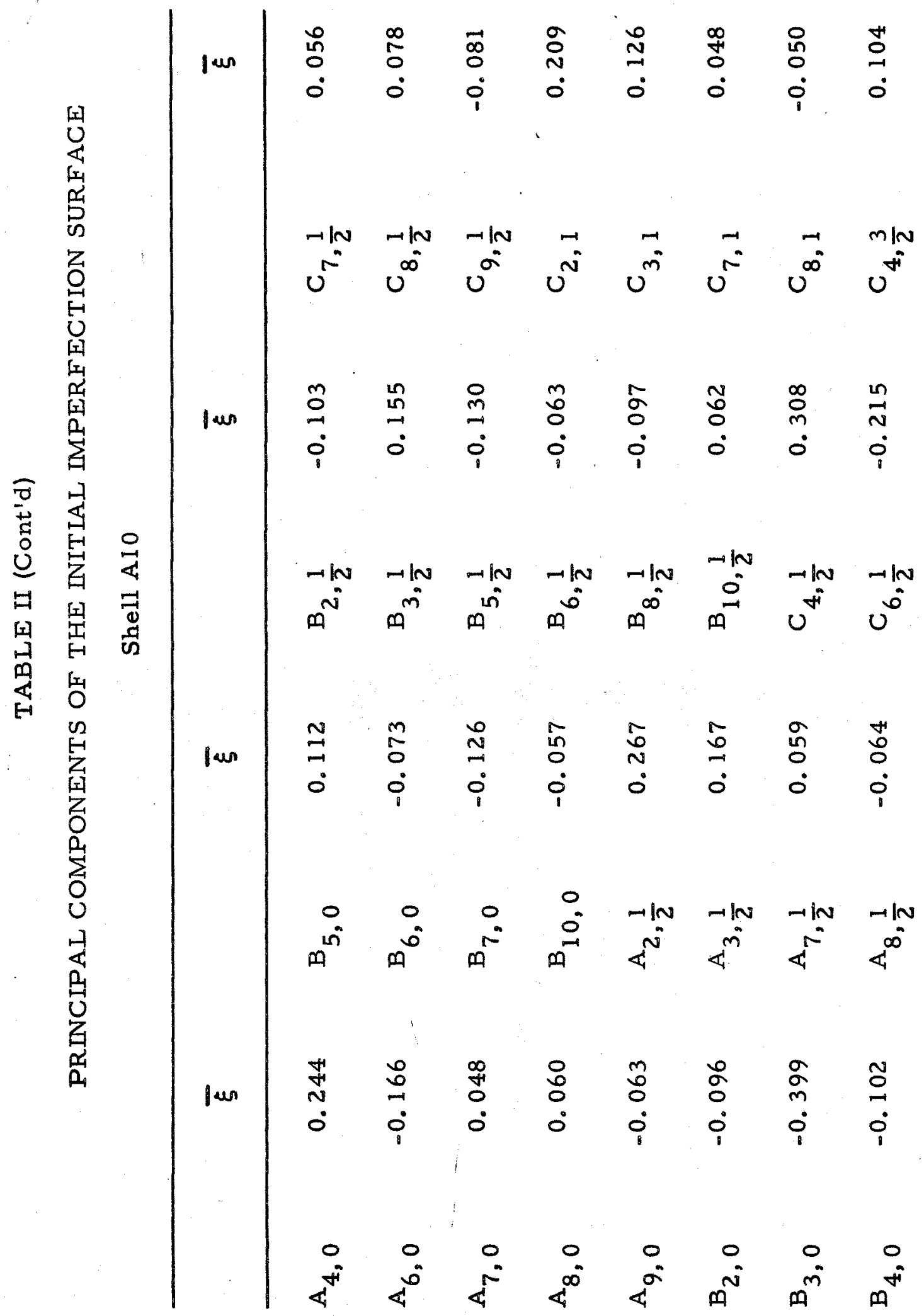


TABLE II (Cont'd)

PRINCIPAL COMPONENTS OF THE INITIAL IMPERFECTION SURFACE

Shell A10

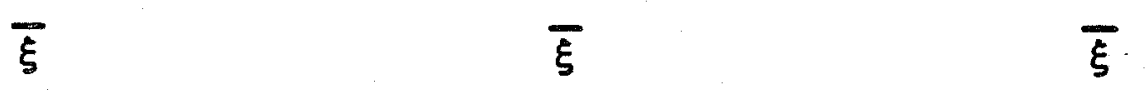

$\begin{array}{llllll}C_{6, \frac{3}{2}} & -0.061 & D_{5, \frac{1}{2}} & 0.143 & D_{8,1} & -0.074 \\ C_{2,2} & 0.094 & D_{6, \frac{1}{2}} & -0.093 & D_{10,1} & 0.047 \\ C_{3,2} & 0.065 & D_{7, \frac{1}{2}} & -0.155 & D_{3, \frac{3}{2}} & -0.159 \\ C_{4, \frac{5}{2}} & 0.059 & D_{10, \frac{1}{2}} & -0.065 & D_{7, \frac{3}{2}} & -0.058 \\ C_{2,3} & 0.057 & D_{2,1} & -0.077 & D_{3,2} & 0.055 \\ D_{2, \frac{1}{2}} & -0.127 & D_{3,1} & 0.120 & D_{5,2} & -0.049 \\ D_{3, \frac{1}{2}} & -0.509 & D_{5,1} & -0.098 & D_{3, \frac{5}{2}} & -0.087 \\ D_{4, \frac{1}{2}} & -0.128 & D_{6,1} & -0.049 & D_{3, \frac{7}{2}} & -0.057\end{array}$




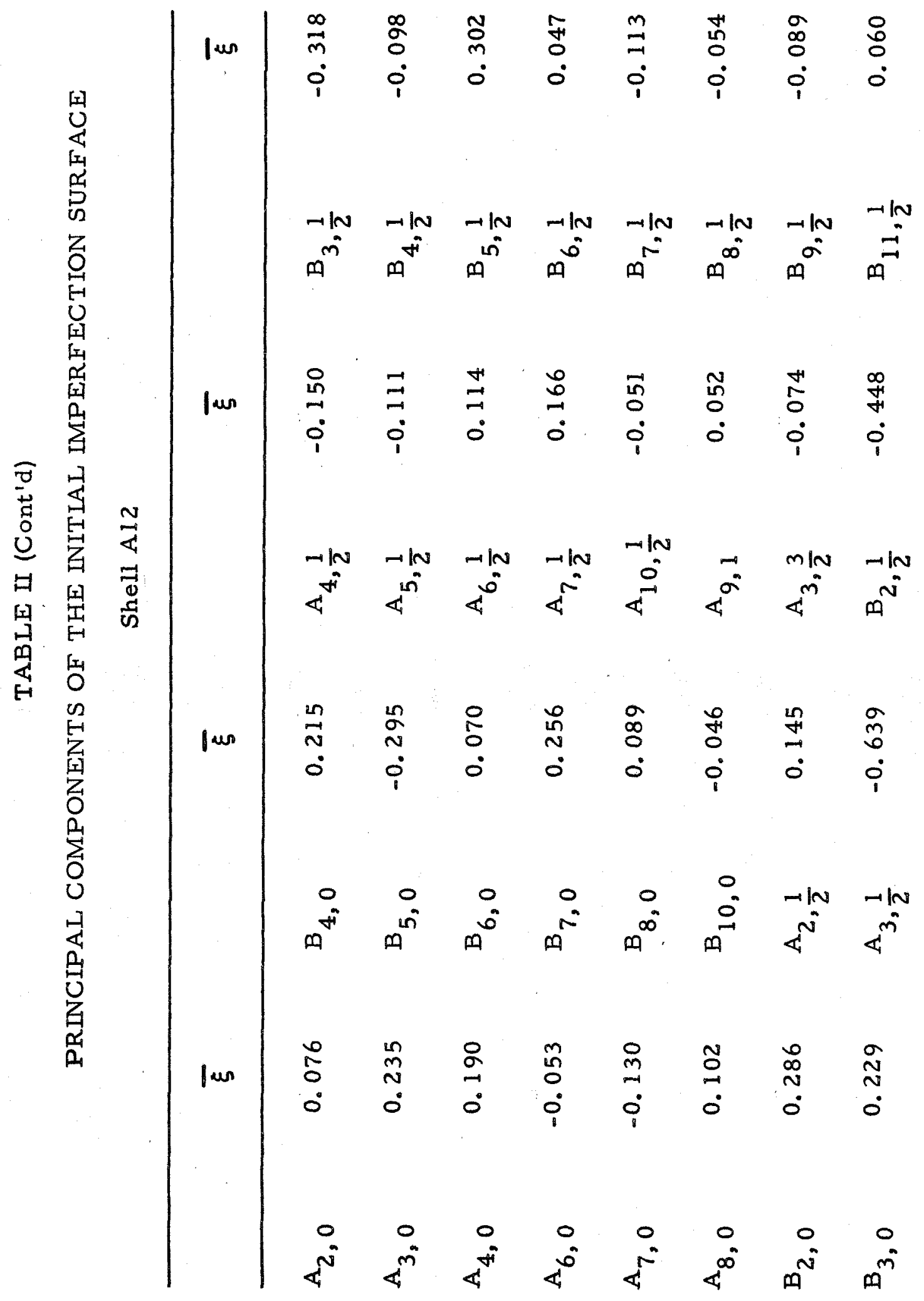




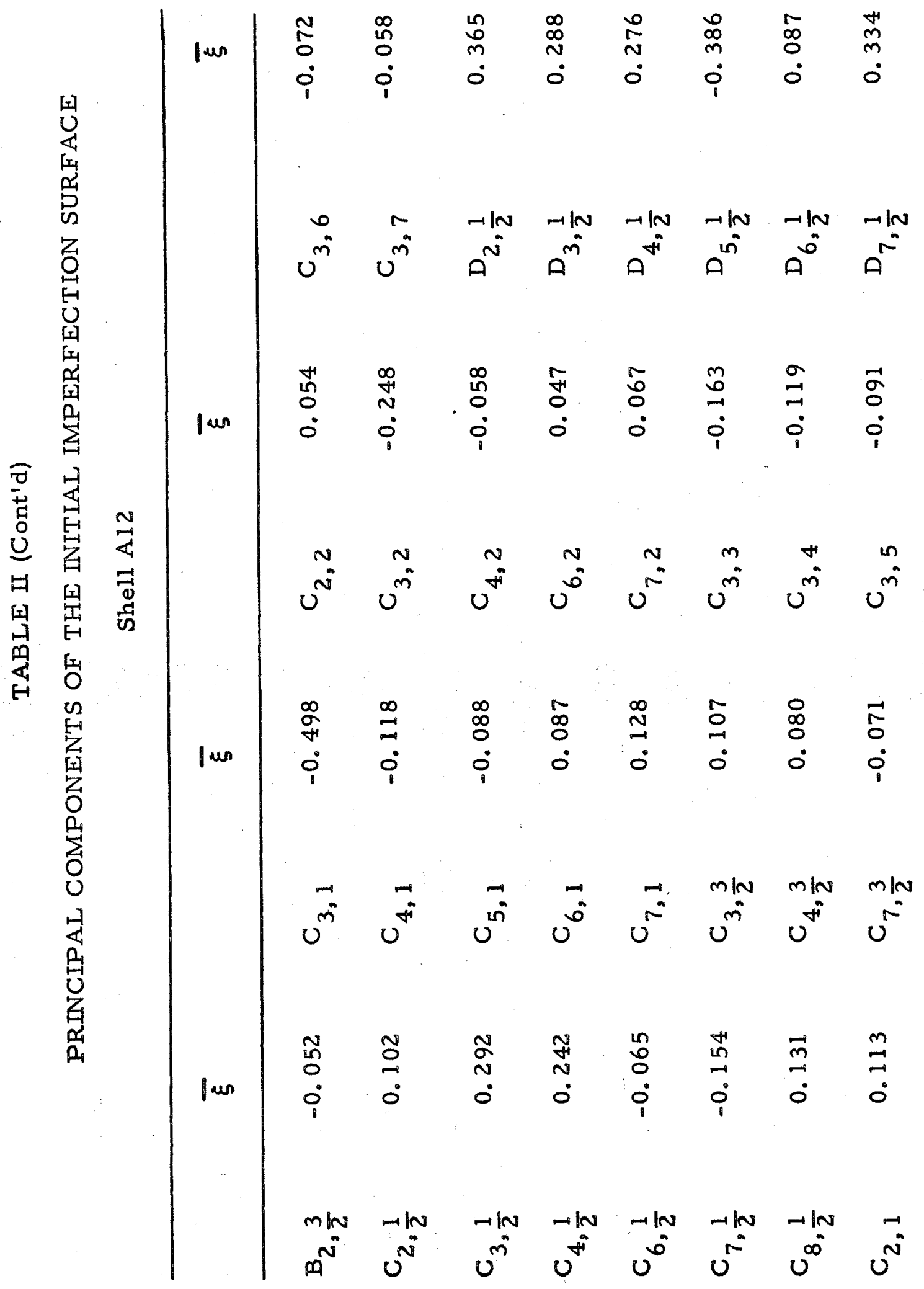




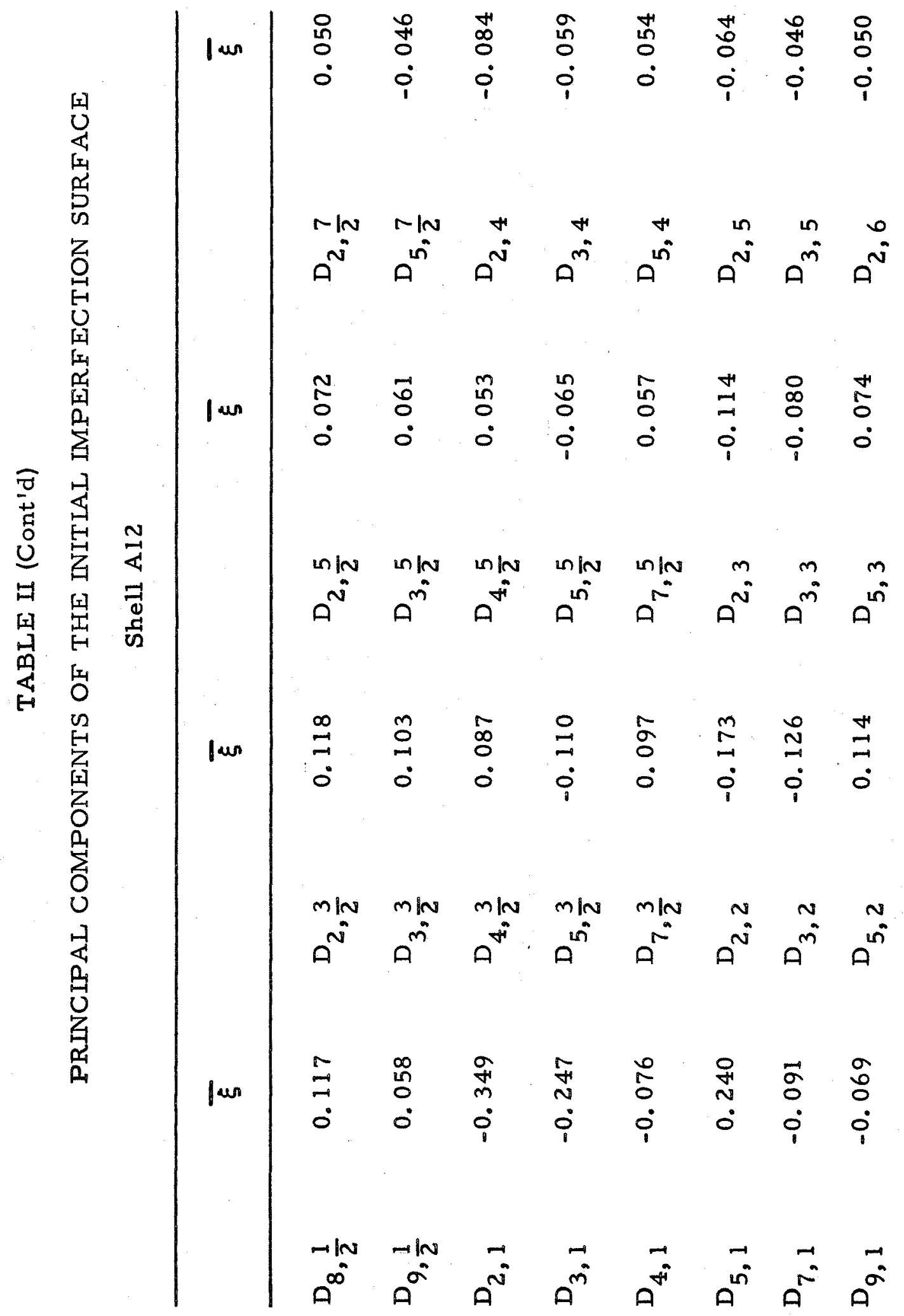


TABLE III

VARIATION OF THE BEST FIT ("PERFECT") CYLINDER

WITH LOAD INCREMENTS

\begin{tabular}{lcccccc} 
Shell A7 & $\begin{array}{l}\mathrm{P} \\
\mathrm{P}_{\mathrm{cl}}\end{array}$ & $\mathrm{X}_{1} \cdot 10^{2}$ & $\mathrm{Y}_{1} \cdot 10^{2}$ & $\epsilon_{1} \cdot 10^{2}$ & $\epsilon_{2} \cdot 10^{2}$ & $\mathrm{R}$ \\
\hline 1 & Inches & Inches & Radians & Radians & Inches \\
\hline 2 & -3.9 & -0.501 & -0.055 & -0.218 & 0.120 & 4.0047 \\
3 & 6.1 & -0.504 & -0.054 & -0.217 & 0.119 & $4.0046 \quad$ J \\
4 & 11.0 & -0.502 & -0.053 & -0.219 & 0.119 & 4.0048 \\
5 & 18.8 & -0.506 & -0.052 & -0.219 & 0.118 & 4.0048 \\
6 & 23.0 & -0.511 & -0.050 & -0.221 & 0.119 & 4.0049 \\
7 & 27.0 & -0.523 & -0.041 & -0.219 & 0.118 & 4.0051 \\
8 & 32.5 & -0.516 & -0.040 & -0.220 & 0.118 & 4.0053
\end{tabular}


TABLE III (Cont'd)

VARIATION OF THE BEST FIT ("PEREECT") CYLINDER

WITH LOAD INCREMENTS

\begin{tabular}{|c|c|c|c|c|c|c|c|}
\hline \multirow[t]{2}{*}{ Shell A7 } & \multirow{2}{*}{\multicolumn{2}{|c|}{$\frac{\mathrm{P}}{\mathrm{P}_{\mathrm{cl}}} \%$}} & \multirow{2}{*}{$\begin{array}{l}x_{1} \cdot 10^{2} \\
\text { Inches }\end{array}$} & \multirow{2}{*}{$\begin{array}{l}Y_{1} \cdot 10^{2} \\
\text { Inches }\end{array}$} & \multirow{2}{*}{$\begin{array}{c}\epsilon_{1} \cdot 10^{2} \\
\text { Radians }\end{array}$} & \multirow{2}{*}{$\begin{array}{c}\epsilon_{2} \cdot 10^{2} \\
\text { Radians }\end{array}$} & \multirow[b]{2}{*}{ Inches } \\
\hline & & & & & & & \\
\hline 9 & & 45.1 & -0.513 & -0.039 & -0.221 & 0.118 & 4.0059 \\
\hline 10 & & 53.9 & -0.520 & -0.041 & -0.223 & 0.118 & 4.0060 \\
\hline 11 & $* *$ & 55.3 & -0.485 & -0.035 & -0.222 & 0.118 & 4.0058 \\
\hline 12 & * & 59.7 & -0.271 & 0.107 & -0.271 & 0.065 & 4.0040 \\
\hline
\end{tabular}

* Buckling Pattern

* Initial Local Buckling Pattern 
TABLE III (Cont'd)

VARLATION OF THE BEST FIT ("PERFECT") CYLINDER

WITH LOAD INCREMENTS

\begin{tabular}{lcccccc}
\hline Shell A8 & $\mathrm{P}_{\mathrm{c} l} \%$ & $\mathrm{X}_{1} \cdot 10^{2}$ & $\mathrm{Y}_{1} \cdot 10^{2}$ & $\epsilon_{1} \cdot 10^{2}$ & $\epsilon_{2} \cdot 10^{2}$ & $\mathrm{R}$ \\
& & Inches & Inches & Radians & Radians & Inches \\
\hline 1 & -2.4 & -0.757 & 0.304 & -0.266 & 0.109 & 4.0036 \\
2 & 4.7 & -0.766 & 0.307 & -0.265 & 0.110 & 4.0033 \\
3 & 11.0 & -0.776 & 0.318 & -0.267 & 0.111 & 4.0034 \\
4 & 20.5 & -0.782 & 0.325 & -0.268 & 0.111 & 4.0034 \\
5 & 27.4 & -0.782 & 0.325 & -0.269 & 0.112 & 4.0034 \\
6 & 33.9 & -0.784 & 0.324 & -0.270 & 0.112 & 4.0034 \\
7 & 42.0 & -0.784 & 0.323 & -0.270 & 0.111 & 4.0035 \\
8 & 48.5 & -0.786 & 0.322 & -0.271 & 0.111 & 4.0036
\end{tabular}




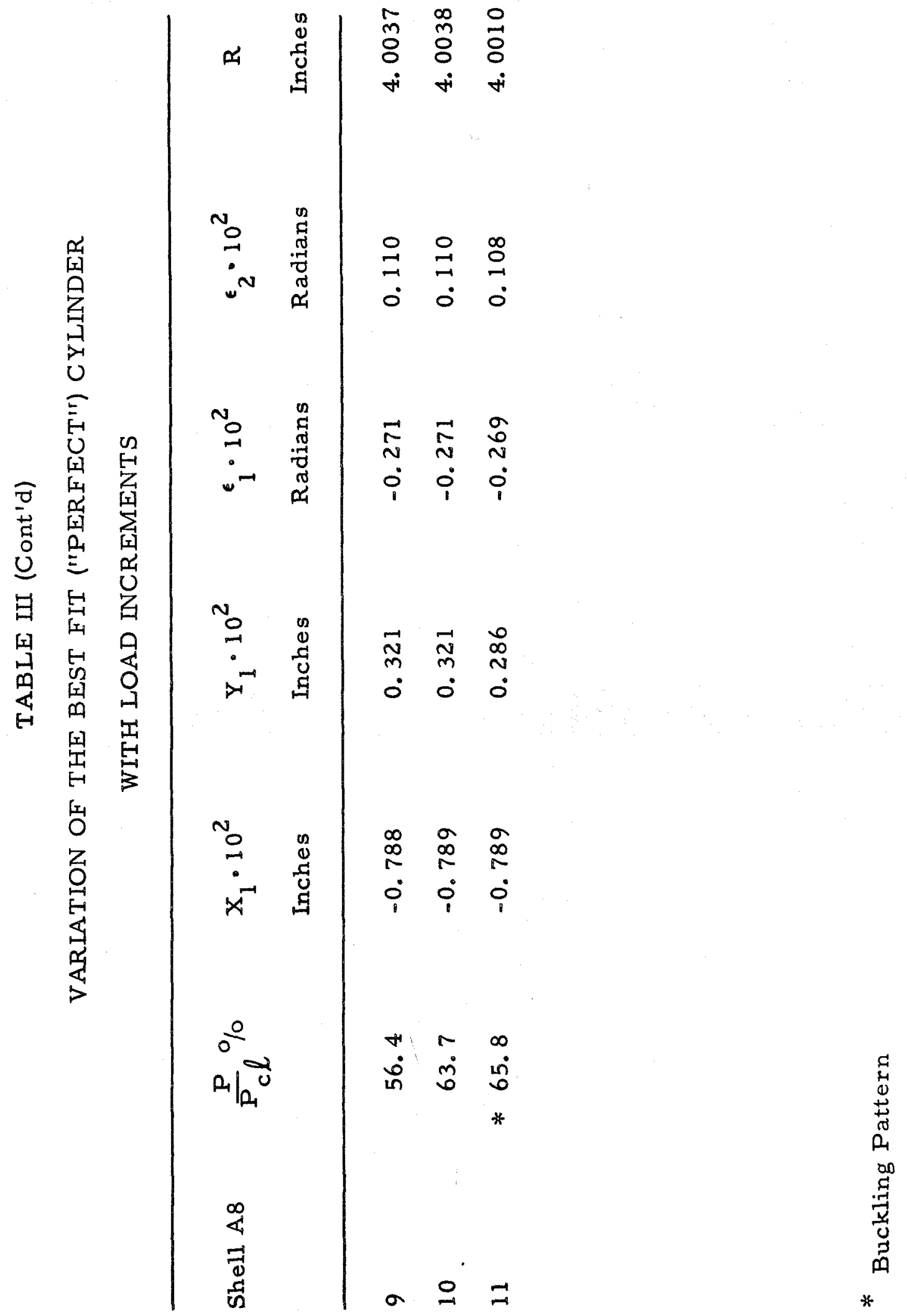


TABLE III (Cont'd)

VARIATION OF THE BEST FIT ("PERFECT") CYLINDER

WITH LOAD INCREMENTS

\begin{tabular}{lcccccc}
\hline Shell A9 & $\mathrm{P}_{\mathrm{cl}} \%$ & $\mathrm{X}_{1} \cdot 10^{2}$ & $\mathrm{Y}_{1} \cdot 10^{2}$ & $\epsilon_{1} \cdot 10^{2}$ & $\epsilon_{2} \cdot 10^{2}$ & $\mathrm{R}$ \\
& & Inches & Inches & Radians & Radians & Inches \\
\hline 1 & -4.5 & -0.594 & 0.346 & -0.213 & 0.101 & 4.0007 \\
2 & 4.2 & -0.599 & 0.348 & -0.210 & 0.100 & 4.0007 \\
3 & 11.8 & -0.602 & 0.349 & -0.210 & 0.100 & 4.0009 \\
4 & 20.9 & -0.603 & 0.350 & -0.209 & 0.101 & 4.0010 \\
5 & 28.6 & -0.604 & 0.352 & -0.209 & 0.101 & 4.0012 \\
6 & 36.0 & -0.605 & 0.353 & -0.209 & 0.101 & 4.0012 \\
7 & 43.5 & -0.605 & 0.353 & -0.209 & 0.101 & 4.0012 \\
8 & 50.4 & -0.605 & 0.354 & -0.208 & 0.101 & 4.0012 \\
9 & 57.1 & -0.607 & 0.355 & -0.207 & 0.101 & 4.0015 \\
10 & 61.4 & -0.605 & 0.355 & -0.208 & 0.102 & 4.0015
\end{tabular}


TABLE IUI (Cont'd)

VARIATION OF THE BEST FIT ("PERFECT") CYLINDER WITH LOAD INCREMENTS

\begin{tabular}{|c|c|c|c|c|c|c|}
\hline \multirow[t]{2}{*}{ Shell A9 } & \multirow{2}{*}{$\frac{\mathrm{P}}{\mathrm{P}_{\mathrm{cl}}} \%$} & $x_{1} \cdot 10^{2}$ & $Y_{1} \cdot 10^{2}$ & $\epsilon_{1} \cdot 10^{2}$ & $\epsilon_{2} \cdot 10^{2}$ & \multirow{2}{*}{$\begin{array}{c}\mathbf{R} \\
\text { Inches }\end{array}$} \\
\hline & & Inches & Inches & Radians & Radians & \\
\hline 11 & 65.6 & -0.605 & 0.355 & -0.209 & 0.102 & 4. 0016 \\
\hline 12 & 69.1 & -0.604 & 0.354 & -0.209 & 0.101 & 4.0016 \\
\hline 13 & 72.2 & -0.604 & 0.353 & -0.209 & 0.101 & 4.0015 \\
\hline 14 & * 73.6 & -0.680 & 0.307 & -0.210 & 0.102 & 3.9989 \\
\hline
\end{tabular}


TABLE III (Cont'd)

VARIATION OF THE BEST FIT ("PERFECT") CYLINDER

WITH LOAD INCREMENTS

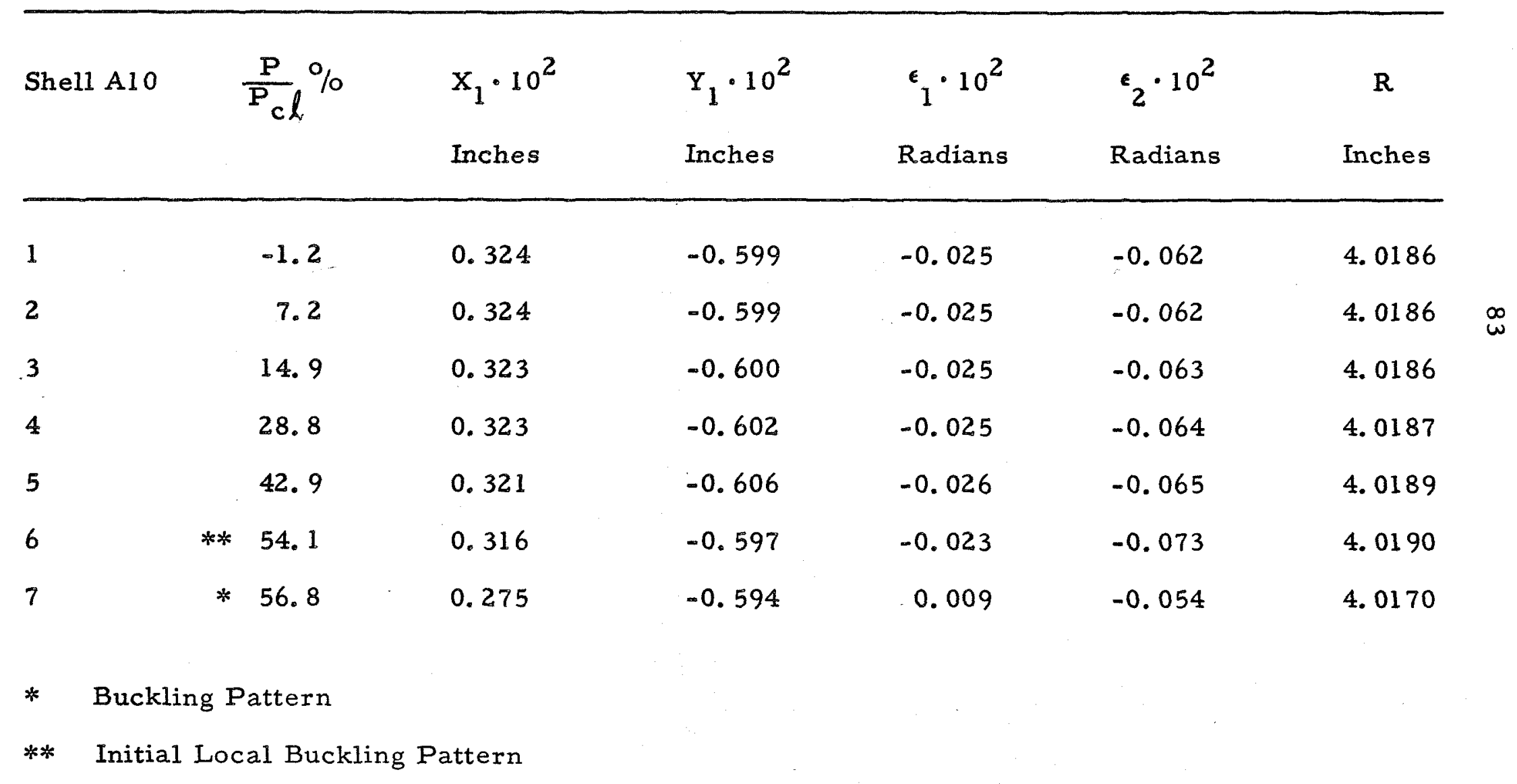


TABLE III (Cont'd)

VARLATION OF THE BEST FIT ("PERFECT") CYLINDER WITH LOAD INCREMENTS

\begin{tabular}{|c|c|c|c|c|c|c|}
\hline Shell A12 & $\frac{\mathrm{P}}{\mathrm{P}_{C l}} \%$ & $x_{1} \cdot 10^{2}$ & $\mathrm{Y}_{1} \cdot 10^{2}$ & $\frac{c}{1} \cdot 10^{2}$ & $\epsilon_{2} \cdot 10^{2}$ & $\mathbf{R}$ \\
\hline & & Inches & Inches & Radians & Radians & Inches \\
\hline 1 & -5.3 & 0.348 & -0.615 & -0.057 & -0.042 & 4. 0087 \\
\hline 2 & 8.1 & 0.349 & -0.622 & -0.058 & -0.041 & 4. 0088 \\
\hline 3 & 24.0 & 0.344 & -0.614 & -0.058 & -0.044 & 4. 0089 \\
\hline 4 & 38.2 & 0.337 & -0.607 & -0.059 & -0.046 & 4. 0091 \\
\hline 5 & 50.5 & 0.338 & -0.622 & -0.062 & -0.045 & 4. 0093 \\
\hline 6 & 60.4 & 0.335 & -0.627 & -0.062 & -0.044 & 4. 0094 \\
\hline 7 & * 67.3 & 0.375 & -0.601 & -0.076 & -0.050 & 4. 0062 \\
\hline
\end{tabular}




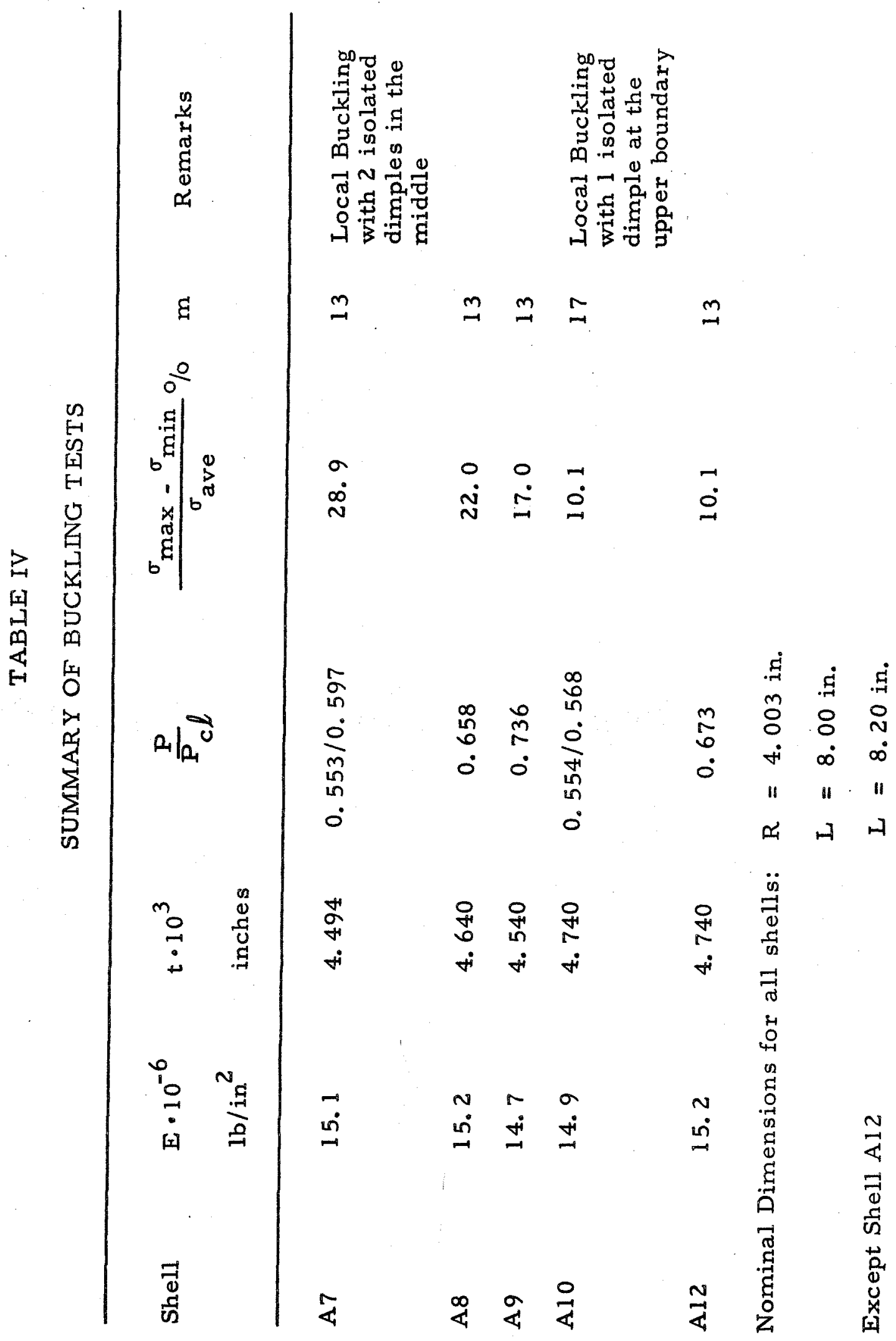


TABLE V

SUMMARY OF "CRITICAL" FOURIER COEFFICIENTS

Shell A7

$\Delta_{9}$

$\bar{\xi}$

$\eta$

$\begin{array}{lccc}\mathrm{A}_{2,0} & 0.056 & -0.402 & -0.14 \\ \mathrm{~A}_{6,0} & -0.015 & 0.114 & -0.13 \\ \mathrm{~A}_{9,0} & 0.026 & 0.031 & 0.84 \\ \mathrm{~A}_{12,0} & 0.011 & 0.005 & 2.20 \\ \mathrm{~B}_{2,0} & -0.024 & -0.241 & 0.10 \\ \mathrm{~B}_{6,0} & -0.011 & -0.165 & 0.07 \\ \mathrm{~B}_{7,0} & 0.019 & -0.074 & -0.26 \\ \mathrm{~B}_{13,0} & -0.018 & -0.026 & 0.69 \\ C_{0,1} & -0.045 & 0.068 & -0.66 \\ C_{0,2} & -0.016 & 0.013 & -1.23\end{array}$

Note: $\bar{\xi}=\frac{\text { Initial Amplitude of the Harmonic }}{\text { Wall Thickness }}$

$$
\eta=\Delta_{9} / \bar{\xi}
$$


TABLE V (Cont'd)

SUMMARY OF "CRITICAL" FOURIER COEFFICIENTS

Shell A7

$\Delta_{9}$

$\bar{\xi}$

$\eta$

$A_{8}, \frac{1}{2}$

0.012

$-0.050$

$-0.24$

$\mathrm{A}_{11, \frac{1}{2}}$

0.014

0.029

0. 48

$\mathrm{A}_{15, \frac{1}{2}}$

0.022

0.011

2. 00

$\mathrm{A}_{9,1}$

$-0.020$

$-0.028$

0.71

$A_{10,1}$

0.012

0.029

0.41

$\mathrm{A}_{18,2}$

$-0.012$

$-0.006$

2. 00

$\mathrm{B}_{2, \frac{1}{2}}$

0.012

$-0.348$

$-0.03$

$B_{11, \frac{1}{2}}$

0.014

0.036

0.39

$B_{12, \frac{1}{2}}$

$-0.021$

$-0.0001$

210.0

$\mathrm{B}_{14, \frac{1}{2}}$

0.028

0.008

3. 50

$B_{1,1}$

$-0.018$

0.013

$-1.38$

$\mathrm{B}_{13,1}$

0.016

0.035

0.46

${ }_{15,1}$

$-0.011$

$-0.013$

0.85 
88

TABLE V (Cont'd)

SUMMARY OF "CRITICAL" FOURIER COEFFICIENTS

Shell A7

$\Delta_{9}$

$\bar{\xi}$

$\eta$

$\mathrm{C}_{2, \frac{1}{2}}$

0.072

$-0.518$

$-0.14$

$c_{6, \frac{1}{2}}$

$-0.019$

0.145

$-0.13$

$\mathrm{C}_{8, \frac{1}{2}}$

0.015

0.150

0.10

$C_{9, \frac{1}{2}}$

0.042

0.051

0.82

$\mathrm{C}_{10, \frac{1}{2}}$

$-0.015$

$-0.073$

0.21

$\mathrm{C}_{8,1}$

0.012

.0 .033

$-0.36$

$\mathrm{C}_{11,1}$

0.016

0.033

0.48

$\mathrm{C}_{15,1}$

0.024

0.016

1. 50

$C_{2, \frac{3}{2}}$

0.019

0.155

$-0.12$

$\mathrm{C}_{2, \frac{5}{2}}$

0.013

$-0.085$

$-0.15$ 
89

TABLE V (Cont'd)

SUMMARY OF "CRITICAL" FOURIER COEFFICIENTS

\begin{tabular}{|c|c|c|c|}
\hline Shell A7 & $\Delta_{9}$ & $\bar{\xi}$ & $\eta$ \\
\hline$D_{2, \frac{1}{2}}$ & -0.029 & -0.308 & 0.09 \\
\hline$D_{3, \frac{1}{2}}$ & -0.011 & 0.189 & -0.06 \\
\hline$D_{6, \frac{1}{2}}$ & -0.016 & -0.200 & 0.08 \\
\hline $\mathrm{D}_{7, \frac{1}{2}}$ & 0.027 & -0.075 & -0.36 \\
\hline $\mathrm{D}_{13, \frac{1}{2}}$ & -0.029 & -0.048 & 0.60 \\
\hline $\mathrm{D}_{11,1}$ & 0.015 & 0.036 & 0.42 \\
\hline $\mathrm{D}_{12,1}$ & -0.022 & -0.036 & 0.61 \\
\hline$D_{14,1}$ & 0.033 & 0.020 & 1.65 \\
\hline$D_{2, \frac{3}{2}}$ & -0.011 & -0.100 & 0.11 \\
\hline$D_{15}, \frac{3}{2}$ & -0.010 & -0.012 & 0.83 \\
\hline
\end{tabular}


TABLE V (Cont'd)

SUMMARY OF "CRITICAL" FOURIER COEFFICIENTS

Shell A8

$\Delta_{9}$

$\bar{\xi}$

$\eta$

$\mathrm{A}_{2,0}$

$-0.023$

$-0.611$

0.04

$A_{5,0}$

0.013

$-0.038$

$-0.34$

$A_{8,0}$

$-0.024$

0.006

$-4.00$

$A_{12,0}$

0.014

$-0.002$

$-7.00$

$A_{13,0}$

0.024

$-0.018$

$-1.33$

$\mathrm{A}_{0, \frac{1}{2}}$

0.014

0.653

0.02

$\mathrm{A}_{0,1}$

0.013

0.103

0.12

$\mathrm{B}_{2,0}$

$-0.026$

0.024

$-1.08$

$\mathrm{B}_{3,0}$

$-0.020$

$-0.042$

0.48

${ }^{B} 8,0$

$-0.012$

$-0.045$

0.27

$B_{10,0}$

0.013

$-0.054$

$-0.24$

$B_{12,0}$

$-0.028$

0.004

$-7.00$

$B_{13,0}$

0.014

0.007

2. 00

$\mathrm{C}_{0,1}$

0.011

0.611

0.02 
TABLE V (Cont'd)

SUMMARY OF "CRITICAL" FOURIER COEFFICIENTS

Shell A8

$\mathrm{A}_{9, \frac{1}{2}}$

$A_{10, \frac{1}{2}}$

$\mathrm{A}_{8,1}$

0.012

$-0.013$

$-0.024$

0.017

${ }^{B} 4, \frac{1}{2}$

$\mathrm{B}_{11, \frac{1}{2}}$

$B_{12,1}$

0.023

$-0.012$

$-0.011$

$\mathrm{B}_{16,1}$

0.018 $\bar{\xi}$

$\eta$

0.010

$-1.20$

0.014

1.00

0.024

0.50

$-0.007$

1.86

$-0.023$

1.05

$-0.154$

$-0.11$

0.025

0.72

0.013

1. 76

$-0.016$

0.75

$-0.003$

3.66 
TABLE V (Cont'd)

SUMMARY OF "CRITICAL" FOURIER COEFFICIENTS

Shell A8

$\Delta 9$

$\bar{\xi}$

$\eta$

$\begin{array}{llll}C_{2, \frac{1}{2}} & -0.031 & -0.777 & 0.04 \\ C_{5, \frac{1}{2}} & 0.017 & -0.035 & -0.48 \\ C_{8, \frac{1}{2}} & -0.036 & -0.005 & 7.20 \\ C_{10, \frac{1}{2}} & -0.015 & -0.014 & 1.07 \\ C_{11, \frac{1}{2}} & -0.011 & -0.001 & 11.00 \\ C_{12, \frac{1}{2}} & 0.024 & 0.0001 & 240.0 \\ C_{13, \frac{1}{2}} & 0.041 & -0.013 & -3.15 \\ C_{9,1} & -0.011 & 0.004 & -2.75 \\ C_{10,1} & 0.015 & 0.015 & 1.00 \\ C_{1, \frac{3}{2}} & 0.010 & 0.051 & 0.20 \\ C_{13, \frac{3}{2}} & -0.011 & -0.022 & 0.50 \\ C_{2, \frac{3}{2}} & -0.011 & -0.239 & 0.05\end{array}$


TABLE V (Cont'd)

SUMMARY OF "CRITICAL" FOURIER COEFFICIENTS

Shell A8

$\Delta_{9}$

$\bar{\xi}$

$\eta$

$\begin{array}{llll}\mathrm{D}_{2, \frac{1}{2}} & -0.036 & 0.277 & -0.13 \\ \mathrm{D}_{3, \frac{1}{2}} & -0.028 & -0.073 & 0.38 \\ \mathrm{D}_{5, \frac{1}{2}} & 0.010 & 0.040 & 0.25 \\ \mathrm{D}_{6, \frac{1}{2}} & 0.015 & 0.148 & 0.10 \\ \mathrm{D}_{7, \frac{1}{2}} & 0.011 & -0.024 & -0.46 \\ \mathrm{D}_{8, \frac{1}{2}} & -0.016 & -0.054 & 0.30 \\ D_{10, \frac{1}{2}} & 0.020 & -0.068 & -0.29 \\ D_{12, \frac{1}{2}} & -0.046 & -0.0004 & 115.0 \\ D_{13, \frac{1}{2}} & 0.022 & 0.016 & 1.38\end{array}$


TABLE V (Cont'd)

SUMMARY OF "CRITICAL" FOURIER COEFFICIENTS

Shell A8

$\Delta_{9}$

$\bar{\xi}$

$\eta$

$\begin{array}{llll}\mathrm{D}_{4,1} & 0.013 & -0.113 & -0.11 \\ \mathrm{D}_{9,1} & -0.011 & -0.048 & 0.23 \\ \mathrm{D}_{11,1} & 0.020 & 0.012 & 1.67 \\ \mathrm{D}_{14,1} & 0.011 & -0.009 & -1.22 \\ \mathrm{D}_{14, \frac{3}{2}} & -0.014 & 0.008 & -1.75 \\ \mathrm{D}_{15, \frac{3}{2}} & 0.011 & 0.002 & 5.50 \\ \mathrm{D}_{16, \frac{3}{2}} & -0.010 & -0.008 & 1.25\end{array}$


95

TABLE V (Cont'd)

SUMMARY OF "CRITICAL" FOURIER COEFFICIENTS

Shell A9

$\Delta_{12}$

$\bar{\xi}$

$\eta$

$\mathrm{A}_{8,0}$

$-0.020$

$-0.004$

5. 00

$\mathrm{A}_{9,0}$

$-0.038$

$-0.061$

0.62

$A_{10,0}$

$-0.026$

$-0.001$

26.00

$\mathrm{A}_{11,0}$

$-0.023$

$-0.002$

11. 50

${ }_{6,0}$

$-0.015$

$-0.005$

3. 00

$B_{10,0}$

0.015

$-0.005$

$-3.00$

$\mathrm{C}_{0,1}$

$-0.013$

0.069

$-0.19$

Note: $\eta=\Delta_{12} / \bar{\xi}$ 
96

TABLE V (Cont'd)

SUMMARY OF "CRITICAL" FOURIER COEFFICIENTS

\section{Shell A9}

$\Delta_{12}$

$\bar{\xi}$

$\eta$

\begin{tabular}{|c|c|c|c|}
\hline$A_{2, \frac{1}{2}}$ & 0.015 & -0.240 & -0.06 \\
\hline$A_{12, \frac{1}{2}}$ & -0.025 & -0.034 & 0.73 \\
\hline$A_{14}, \frac{1}{2}$ & -0.010 & 0.001 & -10.00 \\
\hline$A_{1,1}$ & 0.017 & 0.007 & 2. 43 \\
\hline$A_{9,1}$ & 0.027 & 0.022 & 1.23 \\
\hline$A_{10,1}$ & 0.015 & 0.015 & 1.00 \\
\hline$A_{11,1}$ & 0.023 & 0.015 & 1.53 \\
\hline $\mathrm{A}_{13,1}$ & 0.013 & 0.004 & 3.25 \\
\hline$A_{14,1}$ & 0.014 & 0.005 & 2. 80 \\
\hline$A_{12, \frac{3}{2}}$ & 0.016 & 0.005 & 3.20 \\
\hline $\mathrm{A}_{14}, \frac{3}{2}$ & 0.010 & 0.005 & 2.00 \\
\hline$A_{15, \frac{3}{2}}$ & 0.013 & 0.002 & 6.50 \\
\hline $\mathrm{A}_{17}, \frac{3}{2}$ & 0.010 & 0.001 & 10.00 \\
\hline
\end{tabular}


TABLE V (Gont'd)

SUMMARY OF "CRITICAL" FOURIER COEFFICIENTS

Shell A9

$\Delta_{12} \quad \bar{\xi}$

$\eta$

$\begin{array}{llll}\mathrm{B}_{6, \frac{1}{2}} & 0.011 & 0.035 & 0.31 \\ \mathrm{~B}_{10, \frac{1}{2}} & -0.011 & -0.020 & 0.55 \\ \mathrm{~B}_{12, \frac{1}{2}} & -0.023 & 0.022 & -1.05 \\ \mathrm{~B}_{13, \frac{1}{2}} & -0.014 & -0.019 & 0.74 \\ \mathrm{~B}_{13,1} & -0.019 & -0.008 & 2.37 \\ \mathrm{~B}_{15,1} & -0.011 & 0.002 & -5.50 \\ \mathrm{~B}_{16,1} & -0.012 & -0.009 & 1.33 \\ \mathrm{~B}_{13, \frac{3}{2}} & 0.012 & 0.004 & 3.00 \\ B_{16,2} & 0.011 & 0.002 & 5.50 \\ B_{18,2} & 0.010 & 0.0003 & 33.33\end{array}$


TABLE V (Cont'd)

SUMMARY OF "CRITICAL" FOURIER COEFFICIENTS

Shell A9

$\Delta_{12}$

$\mathrm{C}_{4, \frac{1}{2}}$

$-0.011$

0.014

$-0.79$

$\mathrm{C}_{5, \frac{1}{2}}$

0.011

$-0.052$

$-0.21$

$\mathrm{C}_{8, \frac{1}{2}}$

$-0.029$

$-0.014$

2. 07

$\mathrm{C}_{9, \frac{1}{2}}$

$-0.061$

$-0.087$

0.70

$\mathrm{C}_{10, \frac{1}{2}}$

$-0.039$

$-0.008$

4.87

$C_{11, \frac{1}{2}}$

$-0.039$

$-0.008$

4.87

$\mathrm{C}_{12, \frac{1}{2}}$

$-0.011$

$-0.042$

0.26

$C_{14, \frac{1}{2}}$

$-0.011$

$-0.010$

1. 10

$\mathrm{C}_{2,1}$

0.011

$-0.183$

$-0.06$

$\mathrm{C}_{12,1}$

$-0.030$

$-0.031$

0.97

$\mathrm{C}_{14,1}$

$-0,014$

$-0.002$

7. 00

$\mathrm{C}_{15,1}$

$-0.011$

$-0.006$

1.83

$C_{1}, \frac{3}{2}$

0.011

$-0.001$

$-11.00$

$c_{13, \frac{3}{2}}$

0.011

0.005

2. 20

$c_{14, \frac{3}{2}}$

0.012

0.002

6.00 
TABLE V (Cont'd)

SUMMARY OF "CRITICAL" FOURIER COEFFICIENTS

Shell A9

$\Delta_{12}$

$\bar{\xi}$

$\eta$

\begin{tabular}{|c|c|c|c|}
\hline$D_{6, \frac{1}{2}}$ & -0.019 & -0.013 & 1.46 \\
\hline $\mathrm{D}_{8, \frac{1}{2}}$ & 0.015 & 0.054 & 0.28 \\
\hline$D_{10, \frac{1}{2}}$ & 0.022 & -0.001 & -22.00 \\
\hline $\mathrm{D}_{12, \frac{1}{2}}$ & -0.015 & 0.017 & -0.88 \\
\hline$D_{13, \frac{1}{2}}$ & 0.013 & -0.002 & -6.50 \\
\hline $\mathrm{D}_{10,1}$ & -0.011 & -0.021 & 0.52 \\
\hline $\mathrm{D}_{12, \mathrm{I}}$ & -0.025 & 0.013 & 1.92 \\
\hline $\mathrm{D}_{13,1}$ & -0.018 & -0.018 & 1.00 \\
\hline$D_{13, \frac{3}{2}}$ & -0.016 & -0.010 & 1.60 \\
\hline $\mathrm{D}_{15, \frac{3}{2}}$ & -0.014 & -0.0004 & 35.00 \\
\hline$D_{16}, \frac{3}{2}$ & -0.015 & -0.009 & 1.67 \\
\hline
\end{tabular}


TABLE V (Cont'd)

SUMMARY OF "CRITICAL" FOURIER COEFFICIENTS

Shell Alo

$\Delta_{4}$ $\bar{\xi}$

$\begin{array}{llll}\mathrm{A}_{2,0} & -0.008 & -0.033 & 0.24\end{array}$

$\begin{array}{llll}\mathrm{A}_{3,0} & 0.013 & -0.046 & -0.28\end{array}$

\begin{tabular}{|c|c|c|}
\hline $\mathrm{A}_{4,0}$ & $=0.007$ & 0.244 \\
\hline
\end{tabular}

$\begin{array}{llll}\mathrm{A}_{8,0} & 0.007 & 0.060 & 0.17\end{array}$

$\begin{array}{llll}A_{9,0} & -0.008 & -0.063 & 0.13 \\ A_{13,0} & -0.006 & -0.007 & 0.86\end{array}$

Note: $\eta=\Delta_{4} / \bar{\xi}$ 


\begin{tabular}{|c|c|c|c|}
\hline$\angle L^{\circ} 0^{-}$ & $\varepsilon \mathrm{I0} 0^{\circ} 0$ & $010^{\circ} 0^{-}$ & $I^{b} Z I_{G}$ \\
\hline $29^{\circ} \mathrm{I}-$ & $800^{\circ} 0$ & $\varepsilon \tau 0^{\circ} 0^{-}$ & $\frac{Z}{I}{ }^{\prime} I_{g}$ \\
\hline$\varepsilon \varepsilon^{\circ} 0$ & $810^{\circ} 0^{-}$ & $900^{\circ} 0^{-}$ & $\frac{2}{I}{ }^{7} g$ \\
\hline $70^{\circ} 0$ & SS I 0 & $900^{\circ} 0$ & $\frac{2}{I} \cdot \varepsilon_{g}$ \\
\hline$\varepsilon \varepsilon^{\bullet} Z$ & $\varepsilon 00^{\circ} 0$ & $\angle 00^{\circ} 0$ & $\frac{\tau}{I} \cdot \varepsilon I_{V}$ \\
\hline $50^{\circ} 0^{-}$ & $\angle 9 I^{\circ} 0$ & $800^{\circ} 0^{-}$ & $\frac{z}{I} \cdot \varepsilon_{V}$ \\
\hline$\angle 9^{\circ} z-$ & $\varepsilon 00^{\circ} 0$ & $800^{\circ} 0-$ & $0^{\prime} \varepsilon \mathrm{I}_{\mathrm{g}}$ \\
\hline$\angle 9^{\circ} 0^{-}$ & SI0 0 & $010^{\circ} 0^{-}$ & $0^{\prime} 2 \mathrm{I}_{\mathrm{g}}$ \\
\hline $2 I^{\circ} 0^{-}$ & $\angle 90^{\circ} 0^{-}$ & $\angle 00^{\circ} 0$ & $0^{\circ} 0 \mathrm{I}_{\mathrm{g}}$ \\
\hline $27^{\circ} 0$ & $920^{\circ} 0$ & I I $0^{\circ} 0$ & $0^{\circ} 6 \mathrm{~g}$ \\
\hline $80^{\circ} 0$ & $\varepsilon \angle 0^{\circ} 0^{-}$ & $900^{\circ} 0-$ & $0^{\circ} 9 \mathrm{G}$ \\
\hline$\angle 0^{\circ} 0^{-}$ & $201^{\circ} 0^{-}$ & $\angle 00^{\circ} 0$ & $0^{\circ} \mathrm{g} g$ \\
\hline$\varepsilon 0^{\circ} 0$ & $66 \varepsilon^{\circ} 0^{-}$ & I I0 $0^{\circ}-$ & $0^{\circ} \varepsilon_{\mathrm{g}}$ \\
\hline$S I^{\circ} 0$ & $960^{\circ} 0^{-}$ & I I $0^{\circ} 0^{-}$ & $0^{\circ} z_{\mathrm{g}}$ \\
\hline$u$ & $\underline{\underline{\xi}}$ & ${ }^{\Phi} \nabla$ & OIV IIəЧS \\
\hline
\end{tabular}

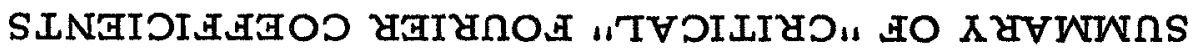
(p,7UoD) $\Lambda$ GTgVL 
TABLE V (Cont'd)

SUMMARY OF "CRITICAL" FOURIER COEFFICIENTS

\section{Shell Alo}

$\Delta_{4}$

$\begin{array}{llll}C_{2, \frac{1}{2}} & -0.010 & -0.043 & 0.23 \\ C_{3, \frac{1}{2}} & 0.018 & -0.003 & -6.00 \\ C_{4, \frac{1}{2}} & -0.008 & 0.308 & -0.03 \\ C_{6, \frac{1}{2}} & -0.007 & -0.215 & 0.03 \\ C_{8, \frac{1}{2}} & 0.011 & 0.078 & 0.14 \\ C_{9, \frac{1}{2}} & -0.012 & -0.081 & 0.15 \\ C_{10, \frac{1}{2}} & 0.009 & -0.007 & -1.29 \\ C_{11, \frac{1}{2}} & 0.008 & -0.014 & -0.57 \\ C_{13, \frac{1}{2}} & -0.009 & -0.015 & 0.60 \\ C_{3,1} & -0.007 & 0.126 & -0.06 \\ C_{13,1} & 0.007 & 0.006 & 1.17\end{array}$


103

TABLE V (Cont'd)

SUMMARY OF "CRITICAL" FOURIER COEFFICIENTS

Shell A10

$\Delta_{4}$

$D_{2}, \frac{1}{2}$

$-0.014$

$-0.127$

0.11

$D_{3, \frac{1}{2}}$

$-0.014$

$-0.509$

0.03

$D_{4}, \frac{1}{2}$

0.009

$-0.129$

$-0.07$

$D_{6, \frac{1}{2}}$

$-0.009$

$-0.093$

0.09

$D_{8}, \frac{1}{2}$

$-0.007$

0.001

$-7.00$

$\mathrm{D}_{9, \frac{1}{2}}$

0.016

0.036

0.45

$\mathrm{D}_{10, \frac{1}{2}}$

0.010

$-0.065$

$-0.15$

$D_{12, \frac{1}{2}}$

$-0.016$

0.013

$-1.23$

$\mathrm{D}_{13, \frac{1}{2}}$

$-0.012$

$-0.001$

12.00 
104

TABLE V (Cont'd)

SUMMARY OF "CRITICAL" FOURIER COEFFICIENTS

Shell A12

$\Delta_{5}$

$\bar{\xi}$

$\eta$

$\mathrm{A}_{2,0}$

0.022

0.076

0.29

$\mathrm{A}_{4,0}$

$-0.014$

0.190

$-0.07$

$A_{5,0}$

0.011

0.011

1.00

$A_{6,0}$

0.014

$-0.053$

$-0.26$

$\mathrm{A}_{8,0}$

$-0.012$

0.102

$-0.12$

$A_{9,0}$

$-0.069$

$-0.011$

6.28

$\mathrm{A}_{10,0}$

$-0.041$

$-0.022$

1. 86

$A_{11,0}$

0.030

0.014

2. 14

$A_{12,0}$

0.016

$-0.027$

$-0.59$

Note: $\eta=\Delta_{5} / \bar{\xi}$ 
TABLE V (Cont'd)

SUMMARY OF "CRITICAL" FOURIER COEFFICIENTS

\begin{tabular}{|c|c|c|c|}
\hline Shell A12 & $\Delta_{5}$ & $\bar{\xi}$ & $\eta$ \\
\hline $\mathrm{B}_{6,0}$ & -0.014 & 0.070 & -0.20 \\
\hline $\mathrm{B}_{10,0}$ & 0.070 & -0.046 & -1.52 \\
\hline $\mathrm{B}_{11,0}$ & 0.063 & -0.024 & -2.63 \\
\hline $\mathrm{B}_{13,0}$ & -0.025 & 0.007 & -3.57 \\
\hline$A_{15}, \frac{1}{2}$ & 0.018 & 0.004 & 4.50 \\
\hline$A_{9,1}$ & 0.044 & 0.052 & 0.85 \\
\hline$A_{10,1}$ & 0.023 & 0.026 & 0.88 \\
\hline$A_{11,1}$ & -0.017 & -0.012 & 1.42 \\
\hline$A_{16,1}$ & 0.024 & -0.016 & -1.50 \\
\hline$A_{17,1}$ & -0.012 & -0.007 & 1.72 \\
\hline $\mathrm{A}_{18,1}$ & -0.017 & 0.004 & -4.25 \\
\hline$A_{15}, \frac{3}{2}$ & -0.012 & -0.005 & 2. 40 \\
\hline$A_{17}, \frac{3}{2}$ & 0.011 & 0.004 & 2.75 \\
\hline
\end{tabular}


TABLE V (Cont'd)

SUMMARY OF "CRITICAL" FOURIER COEFFICIENTS

\section{Shell Al2}

$\Delta_{5}$ $\bar{\xi}$

$\eta$

\begin{tabular}{llll}
\hline$B_{10, \frac{1}{2}}$ & -0.017 & -0.012 & 1.42 \\
$B_{11, \frac{1}{2}}$ & -0.012 & 0.060 & -0.20 \\
$B_{12, \frac{1}{2}}$ & 0.010 & 0.018 & 0.56 \\
$B_{14, \frac{1}{2}}$ & -0.029 & 0.015 & -1.94 \\
$B_{10,1}$ & -0.041 & -0.039 & 1.05 \\
$B_{11,1}$ & -0.041 & -0.042 & 0.98 \\
$B_{15,1}$ & -0.015 & -0.015 & 1.00 \\
$B_{16,1}$ & -0.010 & 0.008 & -1.25 \\
$B_{14, \frac{3}{2}}$ & 0.016 & 0.008 & 2.00 \\
$B_{13,1}$ & 0.018 & 0.028 & 0.64
\end{tabular}


TABLE V (Cont'd)

SUMMARY OF "CRITICAL" FOURIER COEFFICIENTS

Shell A12

$\Delta_{5}$

$\begin{array}{llcc}C_{2, \frac{1}{2}} & 0.029 & 0.102 & 0.28 \\ C_{4, \frac{1}{2}} & -0.020 & 0.242 & -0.08 \\ C_{5, \frac{1}{2}} & 0.016 & -0.004 & -4.00 \\ C_{6, \frac{1}{2}} & 0.022 & -0.065 & -0.34 \\ C_{7, \frac{1}{2}} & 0.012 & -0.154 & -0.08 \\ C_{8, \frac{1}{2}} & -0.018 & 0.131 & -0.14 \\ C_{10, \frac{1}{2}} & -0.063 & -0.040 & 1.58 \\ C_{11}, \frac{1}{2} & 0.045 & 0.023 & 1.96 \\ C_{12, \frac{1}{2}} & 0.023 & -0.027 & 0.85 \\ C_{15, \frac{1}{2}} & 0.011 & -0.001 & -11.00\end{array}$


TABLE V (Cont'd)

SUMMARY OF "CRITICAL" FOURIER COEFFICIENTS

\begin{tabular}{cccc} 
She11 A12 & $\Delta_{5}$ & $\bar{\xi}$ & $\eta$ \\
\hline$C_{11,1}$ & -0.011 & -0.005 & 2.20 \\
$C_{13,1}$ & -0.011 & 0.008 & -1.38 \\
$C_{14,1}$ & -0.013 & -0.004 & 3.25 \\
$C_{15,1}$ & 0.022 & 0.006 & 3.67 \\
$C_{16, \frac{3}{2}}$ & 0.025 & 0.00004 & 625.0 \\
$C_{17, \frac{3}{2}}$ & -0.011 & 0.001 & -11.0 \\
$C_{18, \frac{3}{2}}$ & -0.019 & -0.0003 & 64.40
\end{tabular}


TABLE V (Cont'd)

SUMMARY OF "CRITICAL" FOURIER COEFFICIENTS

\section{Shell A12}

$\Delta_{5}$

$\bar{\xi}$

$\eta$
$\mathrm{D}_{4, \frac{1}{2}}$
0.013
0.276
0.05
$D_{5, \frac{1}{2}}$
$-0.014$
$-0.386$
0.04
$D_{6, \frac{1}{2}}$
$-0.022$
0.087
$-0.25$
$D_{9, \frac{1}{2}}$
0.014
0.058
0.24
$D_{11, \frac{1}{2}}$
0.098
$-0.011$
$-8.90$
$\mathrm{D}_{13, \frac{1}{2}}$
$-0.039$
$-0.004$
9. 75
$\mathrm{D}_{15, \frac{1}{2}}$
0.012
$-0.002$
6.00
$D_{10,1}$
$-0.017$
$-0.011$
1. 55
$\mathrm{D}_{11,1}$
$-0.012$
0.045
$-0.27$
$\mathrm{D}_{12,1}$
0.014
0.012
1. 17
$\mathrm{D}_{14,1}$
$-0.033$
0.008
$-4.12$
$D_{16,1}$
0.011
$-0.001$
$-11.00$
$\mathrm{D}_{16, \frac{3}{2}}$
$-0.013$
0.002
$-6.50$ 


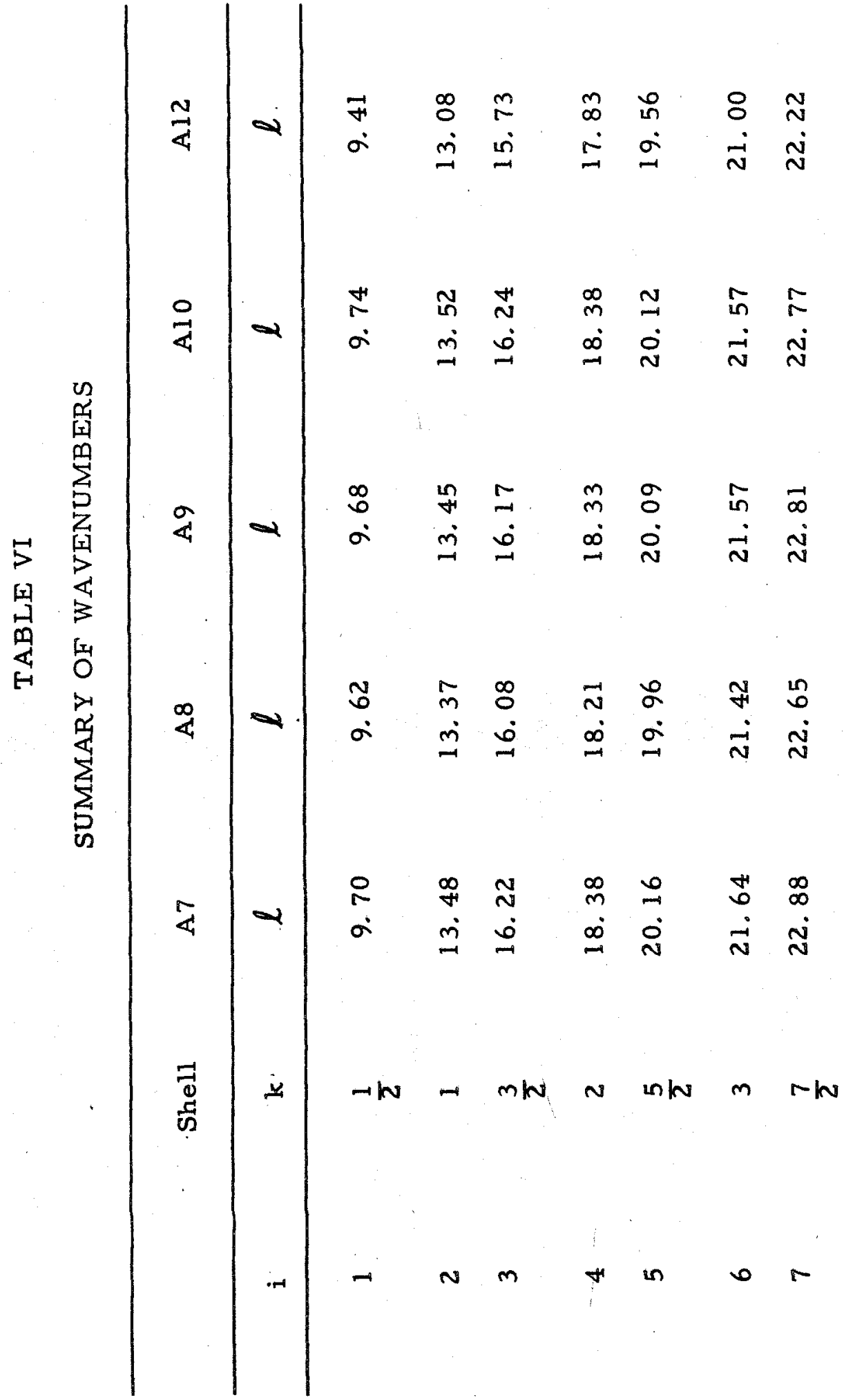


TABLE VII

NUMBERICAL RESULTS (Shell A7)

\begin{tabular}{|c|c|c|c|c|}
\hline \multicolumn{2}{|r|}{$\bar{\xi}_{1}$} & & $\bar{\xi}_{2}$ & $\lambda_{\mathrm{M}}$ \\
\hline \multirow{3}{*}{$A_{0,1}$} & \multirow{3}{*}{-0.0214} & $A_{9}, \frac{1}{2}$ & -0.0050 & 0.998 \\
\hline & & $A_{10, \frac{1}{2}}$ & 0.0402 & 0.840 \\
\hline & & $A_{11, \frac{1}{2}}$ & 0.0292 & 0.956 \\
\hline \multirow{3}{*}{$A_{0,2}$} & \multirow{3}{*}{-0.0421} & $A_{12,1}$ & -0.0135 & 0.992 \\
\hline & & $A_{13,1}$ & 0.0066 & 0.931 \\
\hline & & $A_{14,1}$ & 0.0064 & 0.929 \\
\hline \multirow{3}{*}{$A_{0,3}$} & \multirow{3}{*}{-0.0314} & $A_{15, \frac{3}{2}}$ & -0.0122 & 0.936 \\
\hline & & $A_{16, \frac{3}{2}}$ & -0.0017 & 0.944 \\
\hline & & $A_{17, \frac{3}{2}}$ & 0.0122 & 0.902 \\
\hline \multirow{3}{*}{$A_{0,4}$} & \multirow{3}{*}{-0.0326} & $A_{17,2}$ & 0.0040 & 0.949 \\
\hline & & $\mathrm{A}_{18,2}$ & -0.0061 & 0.905 \\
\hline & & $A_{19,2}$ & 0.0039 & 0.917 \\
\hline \multirow{3}{*}{$A_{0,5}$} & \multirow{3}{*}{-0.0329} & $A_{19, \frac{5}{2}}$ & 0.0032 & 0.920 \\
\hline & & $\mathrm{A}_{20, \frac{5}{2}}$ & -0.0003 & 0.923 \\
\hline & & $A_{21}, \frac{5}{2}$ & -0.0003 & 0.931 \\
\hline
\end{tabular}


TABLE VII (Cont'd)

NUMERICAL RESULTS (Shell A7)

\begin{tabular}{|c|c|c|c|c|}
\hline - & $\bar{\xi}_{1}$ & & $\bar{\xi}_{2}$ & $\lambda_{M}$ \\
\hline \multirow{3}{*}{$A_{0,6}$} & \multirow{3}{*}{-0.0308} & $A_{21,3}$ & -0.0003 & 0.913 \\
\hline & & $A_{22,3}$ & -0.0017 & 0.986 \\
\hline & & $A_{23,3}$ & 0.0003 & 0.925 \\
\hline \multirow{3}{*}{$A_{0,7}$} & \multirow{3}{*}{-0.0423} & $\mathrm{~A}_{22, \frac{7}{2}}$ & -0.0003 & 0.862 \\
\hline & & $\mathrm{A}_{23, \frac{7}{2}}$ & 0.0015 & 0.842 \\
\hline & & $\mathrm{A}_{24, \frac{7}{2}}$ & 0.0002 & 0.862 \\
\hline
\end{tabular}


113

TABLE VII (Cont'd)

NUMERICAL RESULTS (Shell A7)

\begin{tabular}{|c|c|c|c|c|}
\hline \multicolumn{2}{|r|}{$\bar{\xi}_{1}$} & & $\bar{\xi}_{2}$ & $\lambda_{M}$ \\
\hline \multirow{3}{*}{$\mathrm{A}_{0,1}$} & \multirow{3}{*}{-0.0214} & $\mathrm{~B}_{9, \frac{1}{2}}$ & 0.0322 & 0.914 \\
\hline & & $B_{10, \frac{1}{2}}$ & -0.0243 & 0.885 \\
\hline & & $\mathrm{B}_{11, \frac{1}{2}}$ & 0.0359 & 0.933 \\
\hline \multirow{3}{*}{$\mathrm{A}_{0,2}$} & \multirow{3}{*}{-0.0421} & $B_{12,1}$ & -0.0036 & 1.031 \\
\hline & & $B_{13,1}$ & 0.0347 & 0.845 \\
\hline & & $B_{14,1}$ & -0.0091 & 0.916 \\
\hline \multirow{3}{*}{$A_{0,3}$} & \multirow{3}{*}{-0.0314} & $B_{15, \frac{3}{2}}$ & 0.0088 & 0.948 \\
\hline & & $\mathrm{B}_{16, \frac{3}{2}}$ & 0.0079 & 0.912 \\
\hline & & $B_{17, \frac{3}{2}}$ & -0.0053 & 0.933 \\
\hline \multirow{3}{*}{$\mathrm{A}_{0,4}$} & \multirow{3}{*}{-0.0326} & $B_{17,2}$ & 0.0013 & 0.966 \\
\hline & & $\mathrm{B}_{18,2}$ & 0.0035 & 0.918 \\
\hline & & $B_{19,2}$ & 0.0016 & 0.932 \\
\hline \multirow{3}{*}{$\mathrm{A}_{0,5}$} & \multirow{3}{*}{-0.0329} & $\mathrm{~B}_{19, \frac{5}{2}}$ & -0.0012 & 0.924 \\
\hline & & $\mathrm{B}_{20, \frac{5}{2}}$ & 0.0005 & 0.920 \\
\hline & & $\mathrm{B}_{21, \frac{5}{2}}$ & -0.0012 & 0.921 \\
\hline
\end{tabular}


114

TABLE VII (Cont'd)

NUMERICAL RESULTS (Shell A7)

\begin{tabular}{|c|c|c|c|c|}
\hline & $\bar{\xi}_{1}$ & & $\bar{\xi}_{2}$ & $\lambda_{M}$ \\
\hline \multirow{3}{*}{$A_{0,6}$} & \multirow{3}{*}{-0.0308} & $\mathrm{~B}_{21,3}$ & 0.0008 & 0.908 \\
\hline & & $B_{22,3}$ & -0.0003 & 0.910 \\
\hline & & $\mathrm{B}_{23,3}$ & -0.00005 & 0.929 \\
\hline \multirow{3}{*}{$A_{0,7}$} & \multirow{3}{*}{-0.0423} & $B_{22, \frac{7}{2}}$ & -0.0010 & 0.854 \\
\hline & & $\mathrm{B}_{23, \frac{7}{2}}$ & 0.0018 & 0.840 \\
\hline & & $\mathrm{B}_{24, \frac{7}{2}}$ & $-0.8 \cdot 10^{-7}$ & 0.867 \\
\hline
\end{tabular}


115

TABLE VII (Cont'd)

NUMERICAL RESULTS (Shell A7)

\begin{tabular}{|c|c|c|c|c|}
\hline & $\bar{\xi}_{1}$ & & $\bar{\xi}_{2}$ or $\bar{\xi}_{3}$ & $\lambda_{M}$ \\
\hline & & $\mathrm{C}_{9, \frac{1}{2}}$ & 0.0512 & 0.902 \\
\hline \multirow[t]{3}{*}{$\mathrm{c}_{0,1}$} & 0.0683 & $\mathrm{C}_{10, \frac{1}{2}}$ & -0.0734 & 0.794 \\
\hline & & $\mathrm{C}_{11, \frac{1}{2}}$ & 0.0399 & 0.957 \\
\hline & & $\mathrm{A}_{12,1}$ & -0.0135 & 1.016 \\
\hline \multirow[t]{3}{*}{$c_{0,2}$} & 0.0131 & $\mathrm{~A}_{13,1}$ & 0.0066 & 0.955 \\
\hline & & $\mathrm{A}_{14,1}$ & 0.0064 & 0.952 \\
\hline & & $A_{15, \frac{3}{2}}$ & -0.0122 & 0.961 \\
\hline \multirow[t]{3}{*}{$c_{0,3}$} & 0.0101 & $A_{16, \frac{3}{2}}$ & -0.0017 & 0.970 \\
\hline & & $A_{17, \frac{3}{2}}$ & 0.0122 & 0.927 \\
\hline & & $\mathrm{A}_{17,2}$ & 0.0040 & 0.995 \\
\hline \multirow[t]{3}{*}{$c_{0,4}$} & 0.0051 & $A_{18,2}$ & -0.0061 & 0.950 \\
\hline & & $A_{19,2}$ & 0.0039 & 0.962 \\
\hline & & $\mathrm{A}_{19, \frac{5}{2}}$ & 0.0032 & 0.981 \\
\hline \multirow[t]{2}{*}{$c_{0,5}$} & 0.0038 & $\mathrm{~A}_{20, \frac{5}{2}}$ & -0.0003 & 0.986 \\
\hline & & $A_{21}, \frac{5}{2}$ & -0.0003 & 0.995 \\
\hline
\end{tabular}


116

TABLE VII (Cont'd)

NUMERICAL RESULTS (Shell A7)

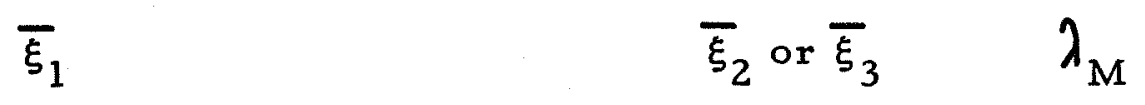

\begin{tabular}{|c|c|c|c|c|}
\hline \multirow{3}{*}{$\mathrm{C}_{0,6}$} & \multirow{3}{*}{0.0013} & $A_{21,3}$ & -0.0003 & 0.994 \\
\hline & & $A_{22,3}$ & -0.0017 & 0.976 \\
\hline & & $A_{23,3}$ & 0.0003 & 1.009 \\
\hline \multirow{3}{*}{$c_{0,7}$} & \multirow{3}{*}{$0.14 \cdot 10^{-6}$} & $A_{22}, \frac{7}{2}$ & -0.0003 & 1.001 \\
\hline & & $\mathrm{A}_{23, \frac{7}{2}}$ & 0.0015 & 0.978 \\
\hline & & $A_{24}, \frac{7}{2}$ & 0.0002 & 1.005 \\
\hline
\end{tabular}


TABLE VII (Cont'd)

NUMERICAL RESULTS (Shell A7)

\begin{tabular}{|c|c|c|c|c|}
\hline & $\bar{\xi}_{1}$ & & $\bar{\xi}_{3}$ & $\lambda_{\mathrm{M}}$ \\
\hline & & ${ }^{B} 9, \frac{1}{2}$. & 0.0322 & 0.893 \\
\hline \multirow[t]{3}{*}{$c_{0,1}$} & 0.0683 & $B_{10, \frac{1}{2}}$ & -0.0243 & 0.823 \\
\hline & & $\mathrm{B}_{11, \frac{1}{2}}$ & 0.0359 & 0.914 \\
\hline & & $B_{12,1}$ & -0.0036 & 1.055 \\
\hline \multirow[t]{3}{*}{$c_{0,2}$} & 0.0131 & $\mathrm{~B}_{13,1}$ & 0.0347 & 0.955 \\
\hline & & $\mathrm{B}_{14,1}$ & -0.0091 & 0.939 \\
\hline & & $B_{15, \frac{3}{2}}$ & 0.0088 & 0.974 \\
\hline \multirow[t]{3}{*}{$c_{0,3}$} & 0.0101 & $\mathrm{~B}_{16, \frac{3}{2}}$ & 0.0079 & 0.970 \\
\hline & & $B_{17, \frac{3}{2}}$ & -0.0053 & 0.959 \\
\hline & & $B_{17,2}$ & 0.00 & 1.012 \\
\hline \multirow[t]{3}{*}{$C_{0,4}$} & 0.0051 & $\mathrm{~B}_{18,2}$ & 0.0035 & 950 \\
\hline & & ${ }^{B}{ }_{19,2}$ & 0. & 0.979 \\
\hline & & $B_{19, \frac{5}{2}}$ & -0.00 & 0.986 \\
\hline \multirow[t]{2}{*}{0,5} & 0.0038 & $B_{20, \frac{5}{2}}$ & 0.0005 & 0.986 \\
\hline & & $B_{21, \frac{5}{2}}$ & -0.0012 & 0.984 \\
\hline
\end{tabular}


TABLE VII (Cont'd)

NUMERICAL RESULTS (Shell A7)

\begin{tabular}{|c|c|c|c|c|}
\hline & $\bar{\xi}_{1}$ & & $\bar{\xi}_{3}$ & $\lambda_{M}$ \\
\hline \multirow{3}{*}{$\mathrm{C}_{0,6}$} & \multirow{3}{*}{0.0013} & $\mathrm{~B}_{21,3}$ & 0.0008 & 0.988 \\
\hline & & $B_{22,3}$ & -0.0003 & 0.976 \\
\hline & & $B_{23,3}$ & -0.00005 & 1.013 \\
\hline \multirow{3}{*}{$C_{0,7}$} & \multirow{3}{*}{$0.14 \cdot 10^{-6}$} & $B_{22, \frac{7}{2}}$ & -0.0010 & 0.991 \\
\hline & & $B_{23, \frac{7}{2}}$ & 0.0018 & 0.978 \\
\hline & & $\mathrm{B}_{24, \frac{7}{2}}$ & $-0.8 \cdot 10^{-7}$ & 1.011 \\
\hline
\end{tabular}


TABLE VII (Cont'd)

NUMBERICAL RESULTS (Shell A8)

$$
\bar{\xi}_{1}
$$

$$
\bar{\xi}_{2} \text { or } \bar{\xi}_{3} \quad \lambda_{M}
$$

\begin{tabular}{|c|c|c|c|c|}
\hline & & $\mathrm{C}_{9, \frac{1}{2}}$ & -0.0248 & 0.889 \\
\hline \multirow[t]{3}{*}{$A_{0,1}$} & 0.1027 & $C_{10, \frac{1}{2}}$ & -0.0145 & 0.888 \\
\hline & & $C_{11, \frac{1}{2}}$ & -0.0009 & 1.117 \\
\hline & & $A_{12,1}$ & -0.0070 & 0.877 \\
\hline \multirow[t]{3}{*}{$A_{0,2}$} & -0.1992 & $\mathrm{~A}_{13,1}$ & -0.0230 & 0.759 \\
\hline & & $A_{14,1}$ & 0.0024 & 0.835 \\
\hline & & $A_{15, \frac{3}{2}}$ & -0.0025 & 0.910 \\
\hline \multirow[t]{3}{*}{$A_{0,3}$} & -0.0796 & $A_{16, \frac{3}{2}}$ & -0.0010 & 0.890 \\
\hline & & $A_{17, \frac{3}{2}}$ & -0.0002 & 0.915 \\
\hline & & $A_{17,2}$ & -0.0026 & 0.872 \\
\hline \multirow[t]{3}{*}{$A_{0,4}$} & -0.0813 & $\mathrm{~A}_{18,2}$ & 0.0006 & 0.859 \\
\hline & & $\mathrm{A}_{19,2}$ & $-0.8 \cdot 10^{-5}$ & 0.875 \\
\hline & & $A_{19}, \frac{5}{2}$ & -0.0029 & 0.856 \\
\hline \multirow[t]{2}{*}{$A_{0,5}$} & -0.0625 & $\mathrm{~A}_{20, \frac{5}{2}}$ & 0.0011 & 0.854 \\
\hline & & $\frac{5}{2}$ & 0.0009 & 0.867 \\
\hline
\end{tabular}


TABLE VII (Cont'd)

NUMERICAL RESULTS (Shell A8)

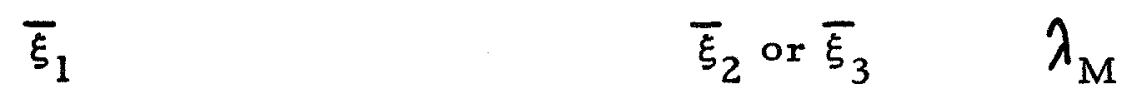

\begin{tabular}{|c|c|c|c|c|}
\hline & & $\mathrm{A}_{21,3}$ & -0.0015 & 0.819 \\
\hline \multirow[t]{3}{*}{$A_{0,6}$} & -0.0637 & $A_{22,3}$ & -0.0026 & 0.812 \\
\hline & & $A_{23,3}$ & 0.0003 & 0.846 \\
\hline & & $A_{22}, \frac{7}{2}$ & 0.0011 & 0.771 \\
\hline \multirow[t]{2}{*}{$\mathrm{A}_{0,7}$} & -0.0716 & $\mathrm{~A}_{23, \frac{7}{2}}$ & 0.0005 & 0.771 \\
\hline & & $\mathrm{A}_{24, \frac{7}{2}}$ & -0.0027 & 0.765 \\
\hline
\end{tabular}


TABLE VII (Cont'd)

NUMERICAL RESULTS (Shell A8)

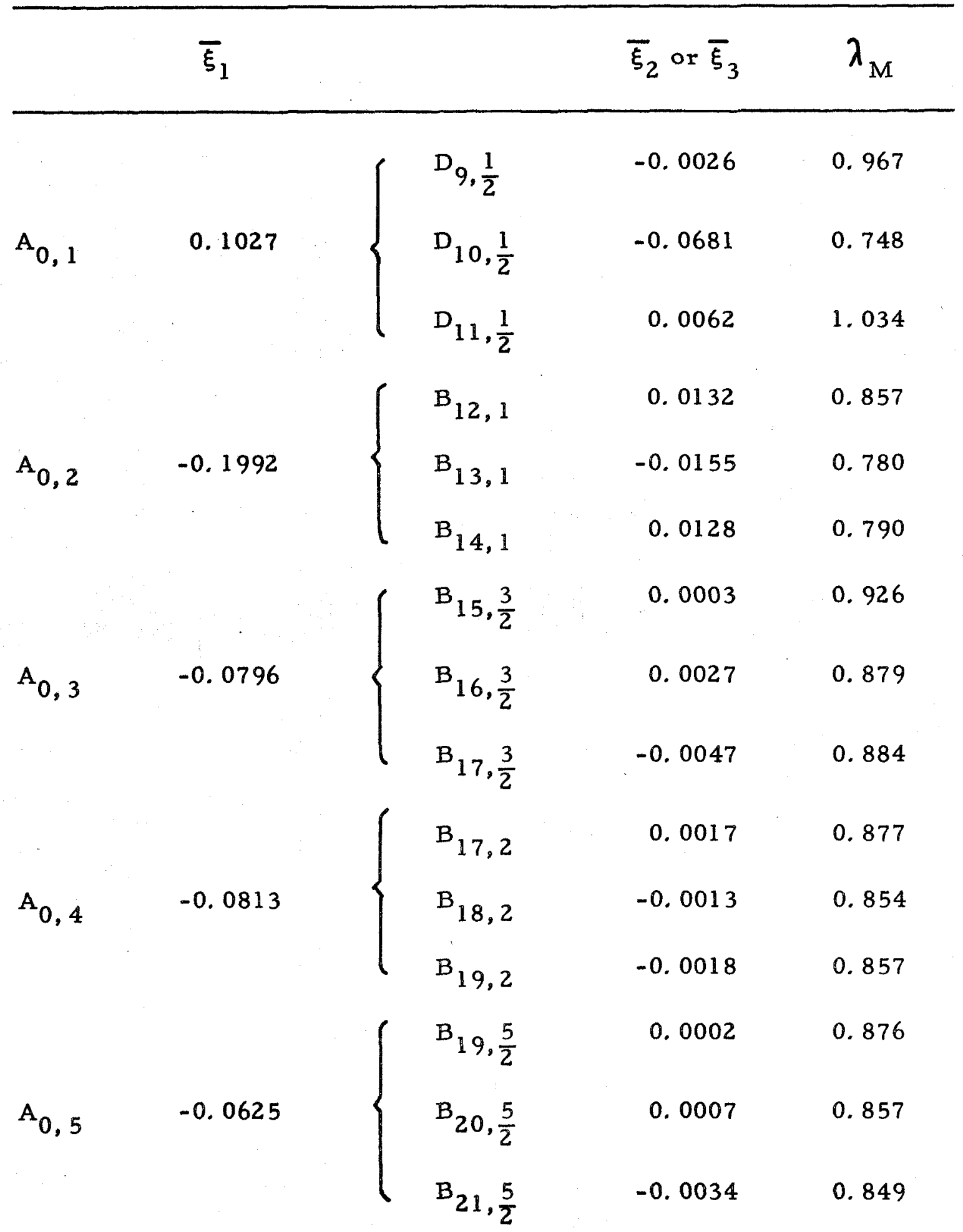


TABLE VII (Cont'd)

NUMERICAL RESULTS (Shell A8)

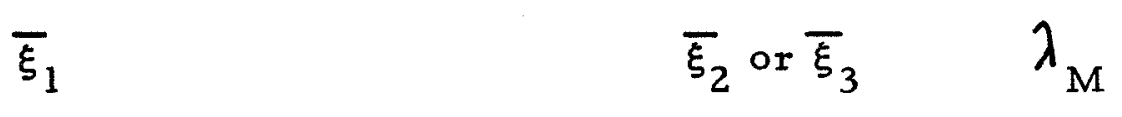

$\mathrm{A}_{0,6} \quad-0.0637$
$\mathrm{~A}_{0,7} \quad \begin{array}{lll}\mathrm{B}_{21,3} & 0.0012 & 0.821 \\ \mathrm{~B}_{22,3} & 0.0010 & 0.823 \\ \mathrm{~B}_{23,3} & 0.0005 & 0.844 \\ \mathrm{~B}_{22, \frac{7}{2}} & 0.0004 & 0.776 \\ \mathrm{~B}_{23, \frac{7}{2}} & -0.0019 & 0.761 \\ \mathrm{~B}_{24, \frac{7}{2}} & 0.3 \cdot 10^{-6} & 0.788\end{array}$


TABLE VII (Cont'd)

NUMERICAI RESULTS (Shell A8)

\begin{tabular}{|c|c|c|c|c|}
\hline - & $\bar{\xi}_{1}$ & & $\bar{\xi}_{2}$ or $\bar{\xi}_{3}$ & $\lambda_{M}$ \\
\hline \multirow{3}{*}{$\mathrm{C}_{0,1}$} & \multirow{3}{*}{0.6106} & $A_{9, \frac{1}{2}}$ & 0.0096 & 0.748 \\
\hline & & $A_{10, \frac{1}{2}}$ & 0.0141 & 0.713 \\
\hline & & $\mathrm{A}_{11, \frac{1}{2}}$ & -0.0157 & 0.798 \\
\hline \multirow{3}{*}{$\mathrm{C}_{0,2}$} & \multirow{3}{*}{0.2661} & $A_{12,1}$ & -0.0070 & 0.828 \\
\hline & & $A_{13,1}$ & -0.0230 & 0.716 \\
\hline & & $\mathrm{A}_{14,1}$ & 0.0024 & 0.789 \\
\hline \multirow{3}{*}{$c_{0,3}$} & \multirow{3}{*}{0.0209} & $\mathrm{~A}_{15, \frac{3}{2}}$ & -0.0025 & 0.981 \\
\hline & & $\mathrm{A}_{16, \frac{3}{2}}$ & -0.0010 & 0.961 \\
\hline & & $A_{17, \frac{3}{2}}$ & -0.0002 & 0.988 \\
\hline \multirow{3}{*}{$\mathrm{C}_{0,4}$} & \multirow{3}{*}{0.0752} & $A_{17,2}$ & -0.0026 & 0.881 \\
\hline & & $A_{18,2}$ & 0.0006 & 0.868 \\
\hline & & $A_{19,2}$ & $-0.8 \cdot 10^{-5}$ & 0.885 \\
\hline \multirow{3}{*}{$\mathrm{C}_{0,5}$} & \multirow{3}{*}{0.0216} & $\mathrm{~A}_{19, \frac{5}{2}}$ & -0.0029 & 0.937 \\
\hline & & $\mathrm{A}_{20, \frac{5}{2}}$ & 0.0011 & 0.937 \\
\hline & & $\mathrm{A}_{21, \frac{5}{2}}$ & 0.0009 & 0.951 \\
\hline
\end{tabular}


124

TABLE VII (Cont'd)

NUMERICAL RESULTS (Shell A8)

\begin{tabular}{|c|c|c|c|c|}
\hline & $\bar{\xi}_{1}$ & . & $\bar{\xi}_{2}$ or $\bar{\xi}_{3}$ & $\lambda_{M}$ \\
\hline \multirow{3}{*}{$C_{0,6}$} & \multirow{3}{*}{0.0075} & $\mathrm{~A}_{21,3}$ & -0.0015 & 0.960 \\
\hline & & $A_{22,3}$ & -0.0026 & 0.952 \\
\hline & & $\mathrm{A}_{23,3}$ & 0.0003 & 0.995 \\
\hline \multirow{3}{*}{$c_{0,7}$} & \multirow{3}{*}{-0.00001} & $C_{22, \frac{7}{2}}$ & -0.0013 & 0.984 \\
\hline & & $\mathrm{C}_{23, \frac{7}{2}}$ & 0.0003 & 0.993 \\
\hline & & $\mathrm{C}_{24, \frac{7}{2}}$ & 0.0003 & 1.009 \\
\hline
\end{tabular}


TABLE VII (Cont'd)

NUMERICAL RESULTS (Shell A8)

\begin{tabular}{|c|c|c|c|c|}
\hline & $\bar{\xi}_{1}$ & & $\bar{\xi}_{2}$ or $\bar{\xi}_{3}$ & $\lambda_{M}$ \\
\hline & & $B_{9, \frac{1}{2}}$ & -0.0551 & 0.651 \\
\hline \multirow[t]{3}{*}{$c_{0,1}$} & 0.6106 & $\mathrm{~B}_{10, \frac{1}{2}}$ & -0.0254 & 0.682 \\
\hline & & $\mathrm{B}_{11, \frac{1}{2}}$ & 0.0098 & 0.821 \\
\hline & & $\mathrm{B}_{12,1}$ & 0.0132 & 0.809 \\
\hline \multirow[t]{3}{*}{$c_{0,2}$} & 0.2661 & $\mathrm{~B}_{13,1}$ & -0.0155 & 0.736 \\
\hline & & $\mathrm{B}_{14,1}$ & 0.0128 & 0.747 \\
\hline & & $B_{15, \frac{3}{2}}$ & 0.0003 & 0.998 \\
\hline \multirow[t]{3}{*}{$\mathrm{C}_{0,3}$} & 0.0209 & $\mathrm{~B}_{16, \frac{3}{2}}$ & 0.0027 & 0.948 \\
\hline & & $\mathrm{B}_{17, \frac{3}{2}}$ & -0.0047 & 0.954 \\
\hline & & $\mathrm{B}_{17,2}$ & 0.0017 & 0.886 \\
\hline \multirow[t]{3}{*}{$C_{0,4}$} & 0.0752 & $\mathrm{~B}_{18,2}$ & -0.0013 & 0.863 \\
\hline & & $B_{19,2}$ & -0.0018 & 0.866 \\
\hline & & $B_{19, \frac{5}{2}}$ & 0.0002 & 0.960 \\
\hline \multirow[t]{2}{*}{$c_{0,5}$} & 0.0216 & $\mathrm{~B}_{20, \frac{5}{2}}$ & 0.0007 & 0.940 \\
\hline & & $B_{21, \frac{5}{2}}$ & -0.0034 & 0.931 \\
\hline
\end{tabular}


126

TABLE VII (Cont'd)

NUMERICAL RESULTS (Shell A8)

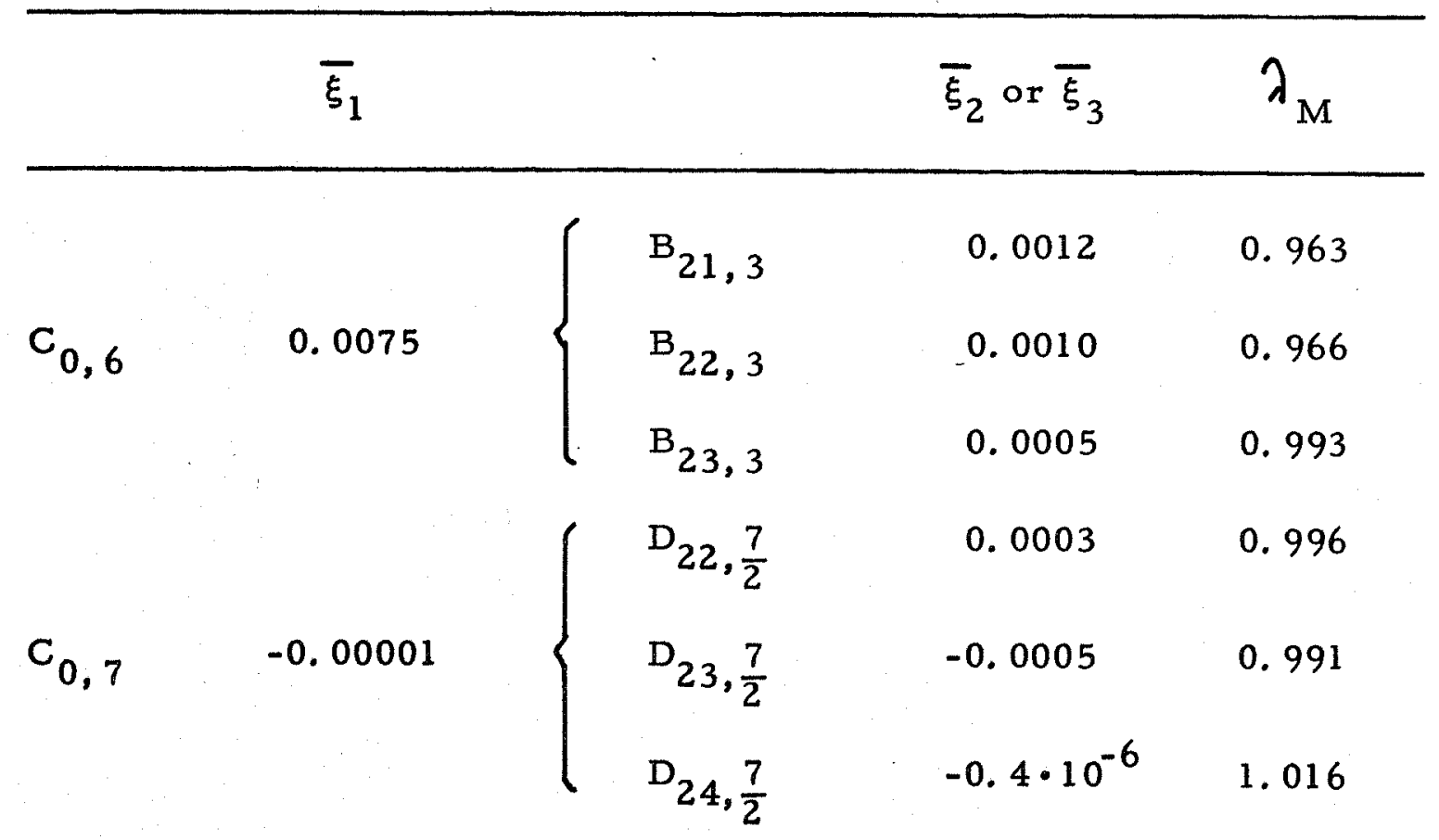


TABLE VII (Cont'd)

NUMERICAL RESULTS (Shell A9)

\begin{tabular}{|c|c|c|c|c|}
\hline & $\bar{\xi}_{1}$ & & $\bar{\xi}_{2}$ or $\bar{\xi}_{3}$ & $\lambda_{\mathrm{M}}$ \\
\hline & & $A_{9, \frac{1}{2}}$ & 0.0149 & 0.947 \\
\hline \multirow[t]{3}{*}{$A_{0,1}$} & -0.0466 & $\mathrm{~A}_{10, \frac{1}{2}}$ & -0.0216 & 0.884 \\
\hline & & $A_{11, \frac{1}{2}}$ & 0.0108 & 1.024 \\
\hline & & $A_{12,1}$ & 0.0055 & 1.010 \\
\hline \multirow[t]{3}{*}{$A_{0,2}$} & -0.0509 & $\mathrm{~A}_{13,1}$ & 0.0044 & 0.933 \\
\hline & & $\mathrm{A}_{14,1}$ & 0.0046 & 0.932 \\
\hline & & $\mathrm{A}_{15, \frac{3}{2}}$ & 0.0020 & 0.974 \\
\hline \multirow[t]{3}{*}{$\mathrm{A}_{0,3}$} & -0.0340 & $\mathrm{~A}_{16, \frac{3}{2}}$ & -0.0005 & 0.951 \\
\hline & & $\mathrm{A}_{17}, \frac{3}{2}$ & 0.0009 & 0.960 \\
\hline & & $A_{17,2}$ & 0.0013 & 0.957 \\
\hline \multirow[t]{3}{*}{$A_{0,4}$} & -0.0365 & $\mathrm{~A}_{18,2}$ & 0.0007 & 0.930 \\
\hline & & $A_{19,2}$ & 0.0004 & 0.937 \\
\hline & & $A_{19, \frac{5}{2}}$ & -0.0019 & 0.913 \\
\hline \multirow[t]{2}{*}{$A_{0,5}$} & -0.0389 & $\mathrm{~A}_{20, \frac{5}{2}}$ & -0.0006 & 0.906 \\
\hline & & $A_{21, \frac{5}{2}}$ & -0.0022 & 0.901 \\
\hline
\end{tabular}


128

TABLE VII (Cont'd)

NUMERICAL RESULTS (Shell A9)

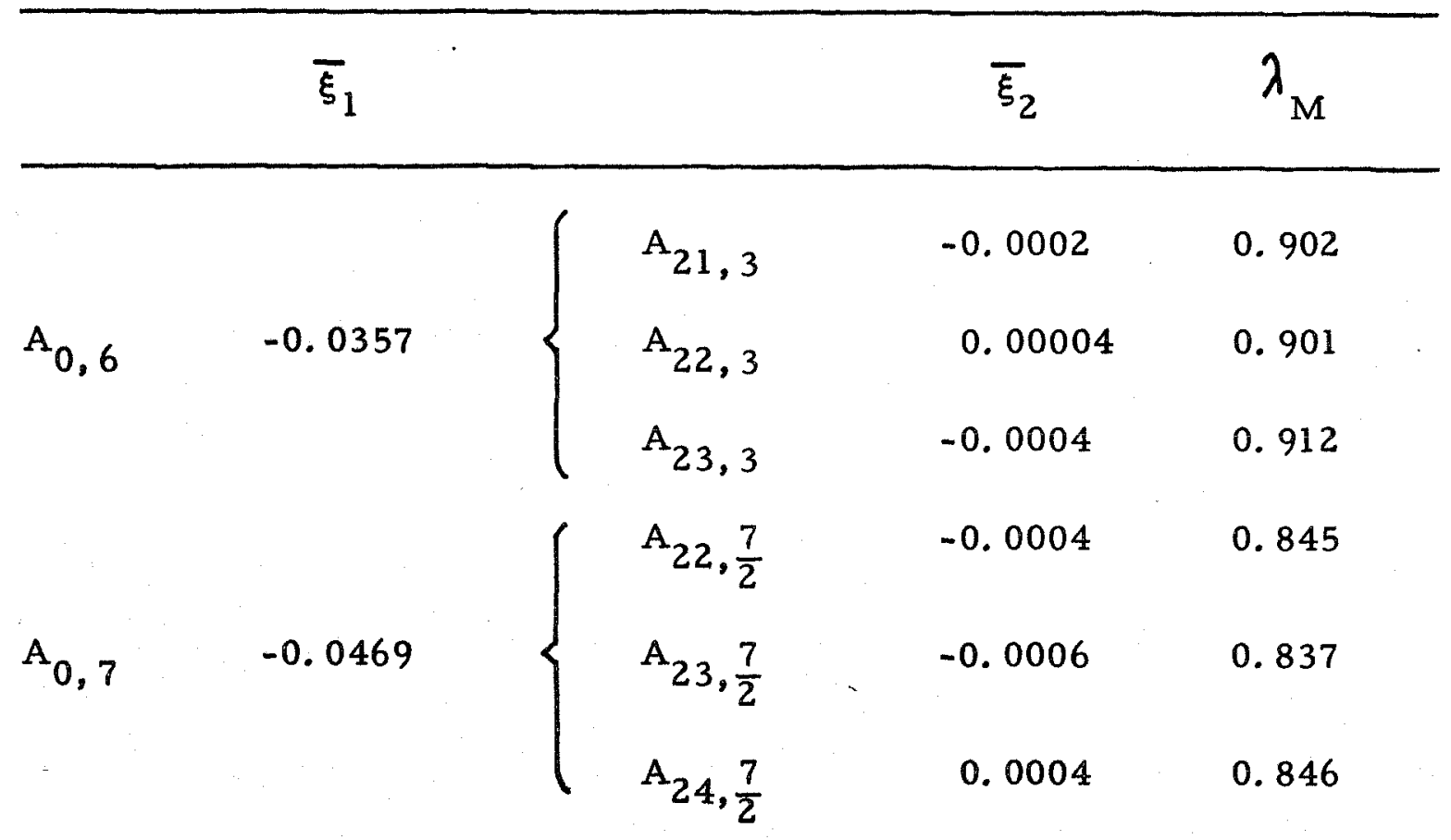


TABLE VII (Cont'd)

NUMERICAL RESULTS (Shell A9)

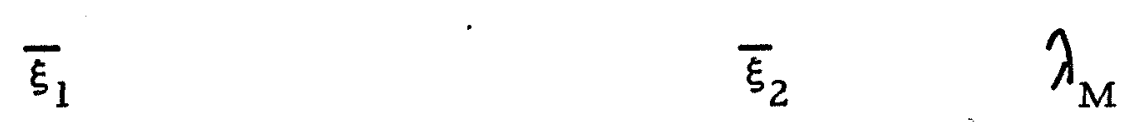

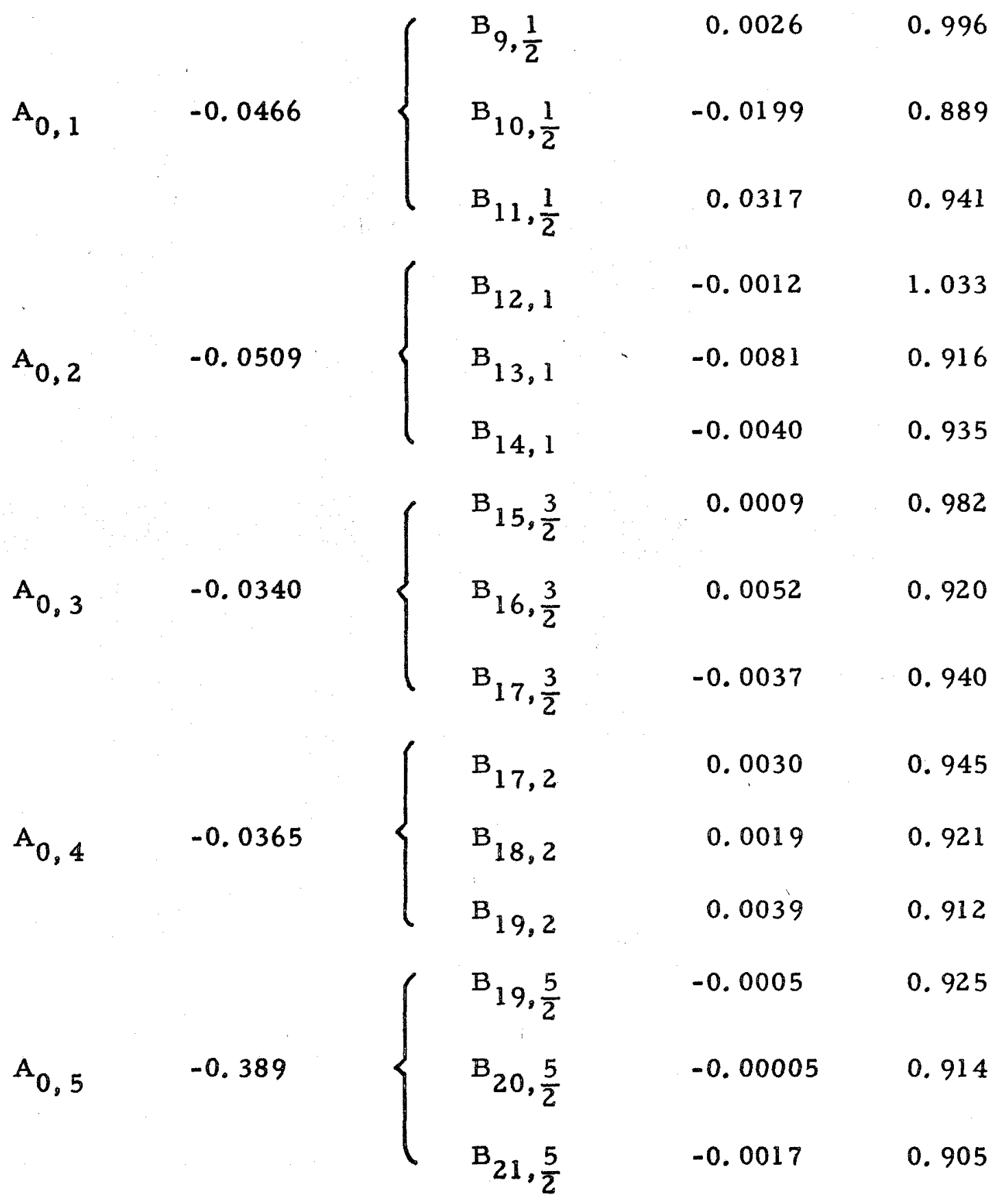


TABLE VII (Cont'd)

NUMERICAL RESULTS (Shell A9)

\begin{tabular}{|c|c|c|c|c|}
\hline \multicolumn{2}{|r|}{$\bar{\xi}_{1}$} & & $\bar{\xi}_{2}$ & $\lambda_{M}$ \\
\hline \multirow{3}{*}{$A_{0,6}$} & \multirow{3}{*}{-0.0357} & $\mathrm{~B}_{21,3}$ & 0.0005 & 0.897 \\
\hline & & $\mathrm{B}_{22,3}$ & -0.0008 & 0.892 \\
\hline & & $B_{23,3}$ & 0.0007 & 0.908 \\
\hline \multirow{3}{*}{$A_{0,7}$} & \multirow{3}{*}{-0.0469} & $\mathrm{~B}_{22, \frac{7}{2}}$ & -0.0008 & 0.841 \\
\hline & & $\mathrm{B}_{23, \frac{7}{2}}$ & -0.0003 & 0.840 \\
\hline & & $\mathrm{B}_{24, \frac{7}{2}}$ & $-0.15 \cdot 10^{-7}$ & 0.854 \\
\hline
\end{tabular}


TABLE VII (Cont'd)

NUMERICAL RESULTS (Shell A9)

\begin{tabular}{|c|c|c|c|c|}
\hline & $\bar{\xi}_{1}$ & & $\bar{\xi}_{3}$ & $\lambda_{M}$ \\
\hline & & $\mathrm{A}_{9, \frac{1}{2}}$ & 0.0149 & 0.937 \\
\hline \multirow[t]{3}{*}{$\mathrm{C}_{0,1}$} & 0.0695 & $\mathrm{~A}_{10, \frac{1}{2}}$ & -0.0216 & 0.875 \\
\hline & & $A_{11}, \frac{1}{2}$ & 0.0108 & 1.014 \\
\hline & & $\mathrm{A}_{12,1}$ & 0.0055 & 1.031 \\
\hline \multirow[t]{3}{*}{$\mathrm{C}_{0,2}$} & 0.0257 & $A_{13,1}$ & 0.0044 & 0.953 \\
\hline & & $A_{14,1}$ & 0.0046 & 0.952 \\
\hline & & $\mathrm{A}_{15, \frac{3}{2}}$ & 0.0020 & 0.989 \\
\hline \multirow[t]{3}{*}{$\mathrm{C}_{0,3}$} & 0.0220 & $A_{16}, \frac{3}{2}$ & -0.0005 & 0.965 \\
\hline & & $A_{17}, \frac{3}{2}$ & 0.0009 & 0.975 \\
\hline & & $A_{17,2}$ & 0.0013 & 1.003 \\
\hline \multirow[t]{3}{*}{$c_{0,4}$} & 0.0086 & $A_{18,2}$ & 0.0007 & 0.977 \\
\hline & & $A_{19,2}$ & 0.0004 & 0.984 \\
\hline & & $A_{19}, \frac{5}{2}$ & -0.0019 & 0.981 \\
\hline \multirow[t]{2}{*}{$\mathrm{C}_{0,5}$} & 0.0067 & $A_{20, \frac{5}{2}}$ & -0.0006 & 0.975 \\
\hline & & $A_{21}, \frac{5}{2}$ & -0.0022 & 0.970 \\
\hline
\end{tabular}


TABLE VII (Cont'd)

NUMERICAL RESULTS (Shell A9)

\begin{tabular}{|c|c|c|c|c|}
\hline & $\bar{\xi}_{1}$ & & $\bar{\xi}_{3}$ & $\lambda_{\mathrm{M}}$ \\
\hline \multirow{3}{*}{$\mathrm{C}_{0,6}$} & \multirow{3}{*}{0.0046} & $A_{21,3}$ & -0.0002 & 0.986 \\
\hline & & $A_{22,3}$ & 0.00004 & 0.986 \\
\hline & & $\mathrm{A}_{23,3}$ & -0.0004 & 0.999 \\
\hline \multirow{3}{*}{$C_{0,7}$} & \multirow{3}{*}{$-0.20 \cdot 10^{-7}$} & $A_{22, \frac{7}{2}}$ & -0.0004 & 0.997 \\
\hline & & $\mathrm{A}_{23, \frac{7}{2}}$ & -0.0006 & 0.998 \\
\hline & & $\mathrm{A}_{24, \frac{7}{2}}$ & 0.0004 & 1.003 \\
\hline
\end{tabular}


TABLE VII (Cont'd)

NUMERICAL RESULTS (Shell A9)

\begin{tabular}{|c|c|c|c|c|}
\hline & $\bar{\xi}_{1}$ & & $\bar{\xi}_{2}$ or $\bar{\xi}_{3}$ & $\lambda_{M}$ \\
\hline & & $C_{9, \frac{1}{2}}$ & -0.0873 & 0.827 \\
\hline \multirow[t]{3}{*}{$C_{0,1}$} & 0.0695 & $C_{10, \frac{1}{2}}$ & -0.0082 & 0.981 \\
\hline & & $C_{11, \frac{1}{2}}$ & -0.0082 & 1.091 \\
\hline & & $\mathrm{B}_{12,1}$ & -0.0012 & 1.054 \\
\hline \multirow[t]{3}{*}{$\mathrm{C}_{0,2}$} & 0.0257 & $\mathrm{~B}_{13,1}$ & -0.0081 & 0.936 \\
\hline & & $\mathrm{B}_{14,1}$ & -0.0040 & 0.956 \\
\hline & & $B_{15, \frac{3}{2}}$ & 0.0009 & 0.997 \\
\hline \multirow[t]{3}{*}{$C_{0,3}$} & 0.0220 & $\mathrm{~B}_{16, \frac{3}{2}}$ & 0.0052 & 0.934 \\
\hline & & $B_{17, \frac{3}{2}}$ & -0.0037 & 0.955 \\
\hline & & $\mathrm{B}_{17,2}$ & 0.0030 & 0.991 \\
\hline \multirow[t]{3}{*}{$C_{0,4}$} & 0.0086 & $\mathrm{~B}_{18,2}$ & 0.0019 & 0.966 \\
\hline & & $B_{19,2}$ & 0.0039 & 0.958 \\
\hline & & $B_{19, \frac{5}{2}}$ & -0.0005 & 0.994 \\
\hline \multirow[t]{2}{*}{$\mathrm{C}_{0,5}$} & 0.0067 & $\mathrm{~B}_{20}, \frac{5}{2}$ & -0.00005 & 0.983 \\
\hline & & $B_{21}, \frac{5}{2}$ & -0.0017 & 0.974 \\
\hline
\end{tabular}


134

TABLE VII (Cont'd)

NUMERICAL RESULTS (Shell A9)

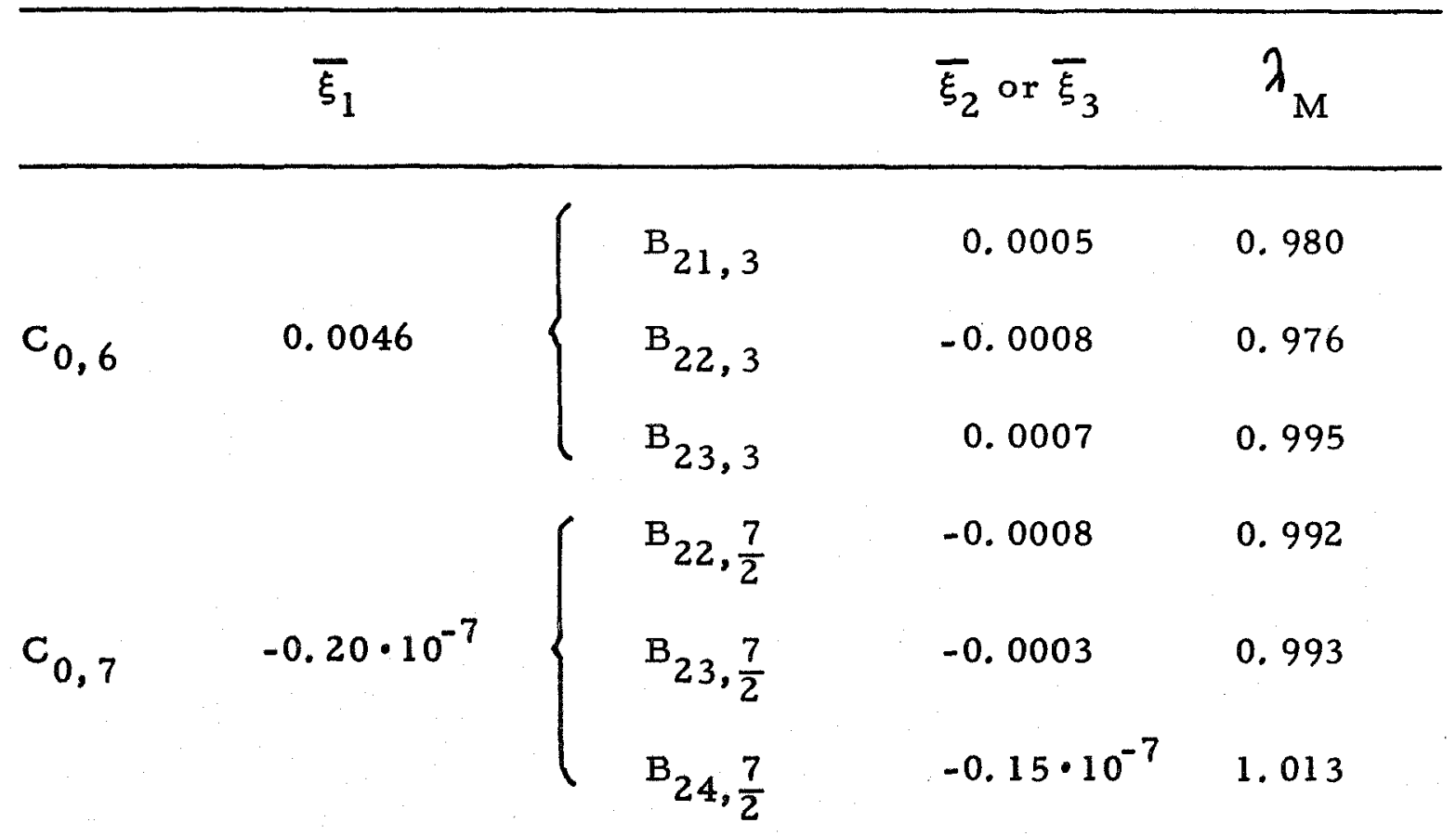


TABLE VII (Cont'd)

NUMERICAL RESULTS (Shell A10)

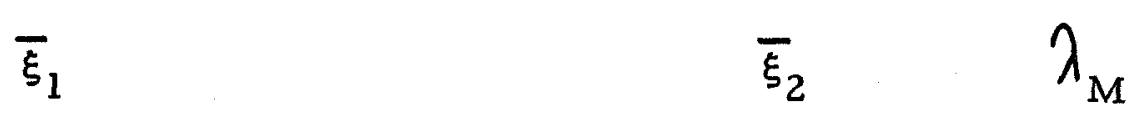

\begin{tabular}{|c|c|c|c|c|}
\hline & & $A_{9, \frac{1}{2}}$ & -0.0035 & 1.007 \\
\hline \multirow[t]{3}{*}{$A_{0,1}$} & -0.0263 & $A_{10, \frac{1}{2}}$ & -0.0134 & 0.918 \\
\hline & & $A_{11, \frac{1}{2}}$ & 0.0316 & 0.939 \\
\hline & & $\mathrm{A}_{12,1}$ & -0.0018 & 1.059 \\
\hline \multirow[t]{3}{*}{$A_{0,2}$} & -0.0231 & $A_{13,1}$ & 0.0119 & 0.926 \\
\hline & & $\mathrm{A}_{14,1}$ & 0.0066 & 0.941 \\
\hline & & $A_{15, \frac{3}{2}}$ & -0.0022 & 0.994 \\
\hline \multirow[t]{3}{*}{$A_{0,3}$} & -0.0185 & $A_{16}, \frac{3}{2}$ & 0.0026 & 0.952 \\
\hline & & $\mathrm{A}_{17, \frac{3}{2}}$ & 0.0020 & 0.966 \\
\hline & & $\mathrm{A}_{17,2}$ & -0.0004 & 0.999 \\
\hline \multirow[t]{3}{*}{$A_{0,4}$} & -0.0156 & $\mathrm{~A}_{18,2}$ & -0.0003 & 0.968 \\
\hline & & $A_{19,2}$ & 0.0015 & 0.959 \\
\hline & & $A_{19, \frac{5}{2}}$ & -0.0004 & 0.972 \\
\hline \multirow[t]{2}{*}{$A_{0,5}$} & -0.0156 & $\mathrm{~A}_{20, \frac{5}{2}}$ & -0.0002 & 0.957 \\
\hline & & $A_{21, \frac{5}{2}}$ & 0.00007 & 0.96 \\
\hline
\end{tabular}


136

TABLE VII (Cont'd)

NUMERICAL RESULTS (Shell Al0)

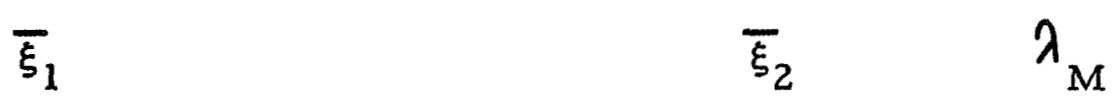

$\mathrm{A}_{0,6}-0.0176 \quad\left\{\begin{array}{lll}\mathrm{A}_{21,3} & 0.0009 & 0.936 \\ \mathrm{~A}_{22,3} & 0.0018 & 0.925 \\ \mathrm{~A}_{23,3} & 0.00008 & 0.961\end{array}\right.$
$\mathrm{A}_{0,7}-0.0204\left\{\begin{array}{lll}\mathrm{A}_{22, \frac{7}{2}} & -0.0005 & 0.916 \\ \mathrm{~A}_{23, \frac{7}{2}} & 0.0001 & 0.915 \\ \mathrm{~A}_{24, \frac{7}{2}} & -0.0009 & 0.914\end{array}\right.$


TABLE VII (Cont'd)

NUMERICAL RESULTS (Shell A10)

\begin{tabular}{|c|c|c|c|c|}
\hline & $\bar{\xi}_{1}$ & & $\bar{\xi}_{2}$ & $\lambda_{M}$ \\
\hline & & ${ }^{B} 9, \frac{1}{2}$ & 0.0026 & 1.012 \\
\hline \multirow[t]{3}{*}{$A_{0,1}$} & -0.0263 & $\mathrm{~B}_{10, \frac{1}{2}}$ & 0.0616 & 0.786 \\
\hline & & $B_{11, \frac{1}{2}}$ & 0.0158 & 0.998 \\
\hline & & $\mathrm{B}_{12,1}$ & 0.0131 & 1.011 \\
\hline \multirow[t]{3}{*}{$A_{0,2}$} & -0.0231 & $\mathrm{~B}_{13,1}$ & 0.0106 & 0.930 \\
\hline & & $\mathrm{B}_{14,1}$ & 0.0001 & 0.985 \\
\hline & & $B_{15, \frac{3}{2}}$ & 0.0031 & 0.988 \\
\hline \multirow[t]{3}{*}{$\mathrm{A}_{0,3}$} & -0.0185 & $B_{16, \frac{3}{2}}$ & 0.0018 & 0.958 \\
\hline & & $B_{17, \frac{3}{2}}$ & 0.0029 & 0.960 \\
\hline & & $\mathrm{B}_{17,2}$ & 0.0027 & 0.981 \\
\hline \multirow[t]{3}{*}{$\mathrm{A}_{0,4}$} & -0.0156 & $\mathrm{~B}_{18,2}$ & 0.0010 & 0.960 \\
\hline & & $B_{19,2}$ & -0.0008 & 0.965 \\
\hline & & $B_{19, \frac{5}{2}}$ & 0.0011 & 0.965 \\
\hline \multirow[t]{2}{*}{$A_{0,5}$} & -0.0156 & $\mathrm{~B}_{20, \frac{5}{2}}$ & 0.0004 & 0.955 \\
\hline & & $\mathrm{B}_{21, \frac{5}{2}}$ & $0.8 \cdot 10^{-5}$ & 0.971 \\
\hline
\end{tabular}


TABLE VII (Cont'd)

NUMERICAL RESULTS (Shell Al0)

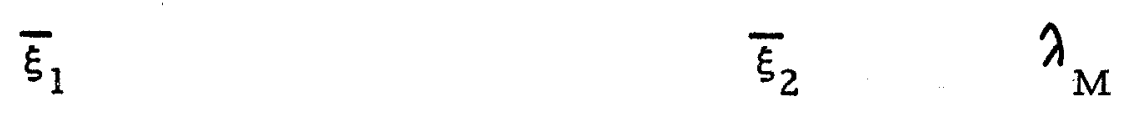

$\mathrm{A}_{0,6}-0.0176 \quad\left\{\begin{array}{lll}\mathrm{B}_{21,3} & 0.0003 & 0.943 \\ \mathrm{~B}_{22,3} & 0.0002 & 0.941 \\ \mathrm{~B}_{23,3} & -0.0013 & 0.945 \\ \mathrm{~A}_{0,7} & -0.0204 \\ \mathrm{~B}_{22, \frac{7}{2}} & -0.0002 & 0.919 \\ \mathrm{~B}_{23, \frac{7}{2}} & 0.0006 & 0.908 \\ \mathrm{~B}_{24, \frac{7}{2}} & 0.3 \cdot 10^{-8} & 0.929\end{array}\right.$


TABLE VII (Cont'd)

NUMERICAL RESULTS (Shell A.10)

\begin{tabular}{|c|c|c|c|c|}
\hline & $\bar{\xi}_{1}$ & & $\bar{\xi}_{2}$ & $\lambda_{M}$ \\
\hline & & $C_{9, \frac{1}{2}}$ & -0.0813 & 0.813 \\
\hline \multirow[t]{3}{*}{$\mathrm{C}_{0,1}$} & -0.0209 & $c_{10, \frac{1}{2}}$ & -0.0072 & 0.946 \\
\hline & & $C_{11, \frac{1}{2}}$ & -0.0139 & 1.009 \\
\hline & & $A_{12,1}$ & -0.0018 & 1.077 \\
\hline \multirow[t]{3}{*}{$\mathrm{C}_{0,2}$} & 0.0040 & $A_{13,1}$ & 0.0119 & 0.942 \\
\hline & & $A_{14,1}$ & 0.0066 & 0.957 \\
\hline & & $A_{15, \frac{3}{2}}$ & -0.0022 & 1.012 \\
\hline \multirow[t]{3}{*}{$c_{0,3}$} & 0.0051 & $A_{16}, \frac{3}{2}$ & 0.0026 & 0.970 \\
\hline & & $A_{17, \frac{3}{2}}$ & 0.0020 & 0.984 \\
\hline & & $\mathrm{A}_{17,2}$ & -0.0004 & 1.023 \\
\hline \multirow[t]{3}{*}{$\mathrm{c}_{0,4}$} & 0.0029 & $A_{18,2}$ & -0.0003 & 0.991 \\
\hline & & $\mathrm{A}_{19,2}$ & 0.0015 & 0.983 \\
\hline & & $A_{19, \frac{5}{2}}$ & -0.0004 & 1.007 \\
\hline \multirow[t]{2}{*}{$c_{0,5}$} & 0.0014 & $A_{20, \frac{5}{2}}$ & -0.0002 & 0.991 \\
\hline & & $A_{21}, \frac{5}{2}$ & 0.00007 & 1.004 \\
\hline
\end{tabular}


TABLE VII (Cont'd)

NUMERICAL RESULTS (Shell A10)

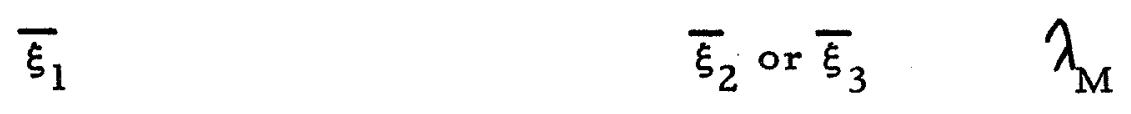

$\begin{array}{ll}\mathrm{C}_{0,6} & 0.0005\end{array}\left\{\begin{array}{lll}\mathrm{A}_{21,3} & 0.0009 & 0.988 \\ \mathrm{~A}_{22,3} & 0.0018 & 0.977 \\ \mathrm{~A}_{23,3} & 0.00008 & 1.017\end{array}\right\}$


TABLE VII (Cont'd)

NUMERICAL RESULTS (Shell Al0)

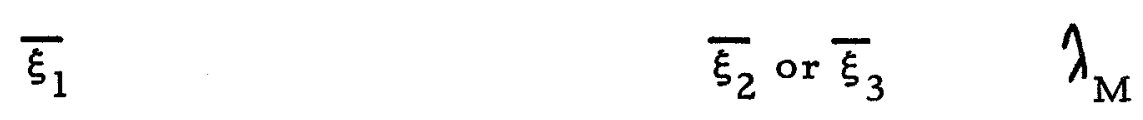

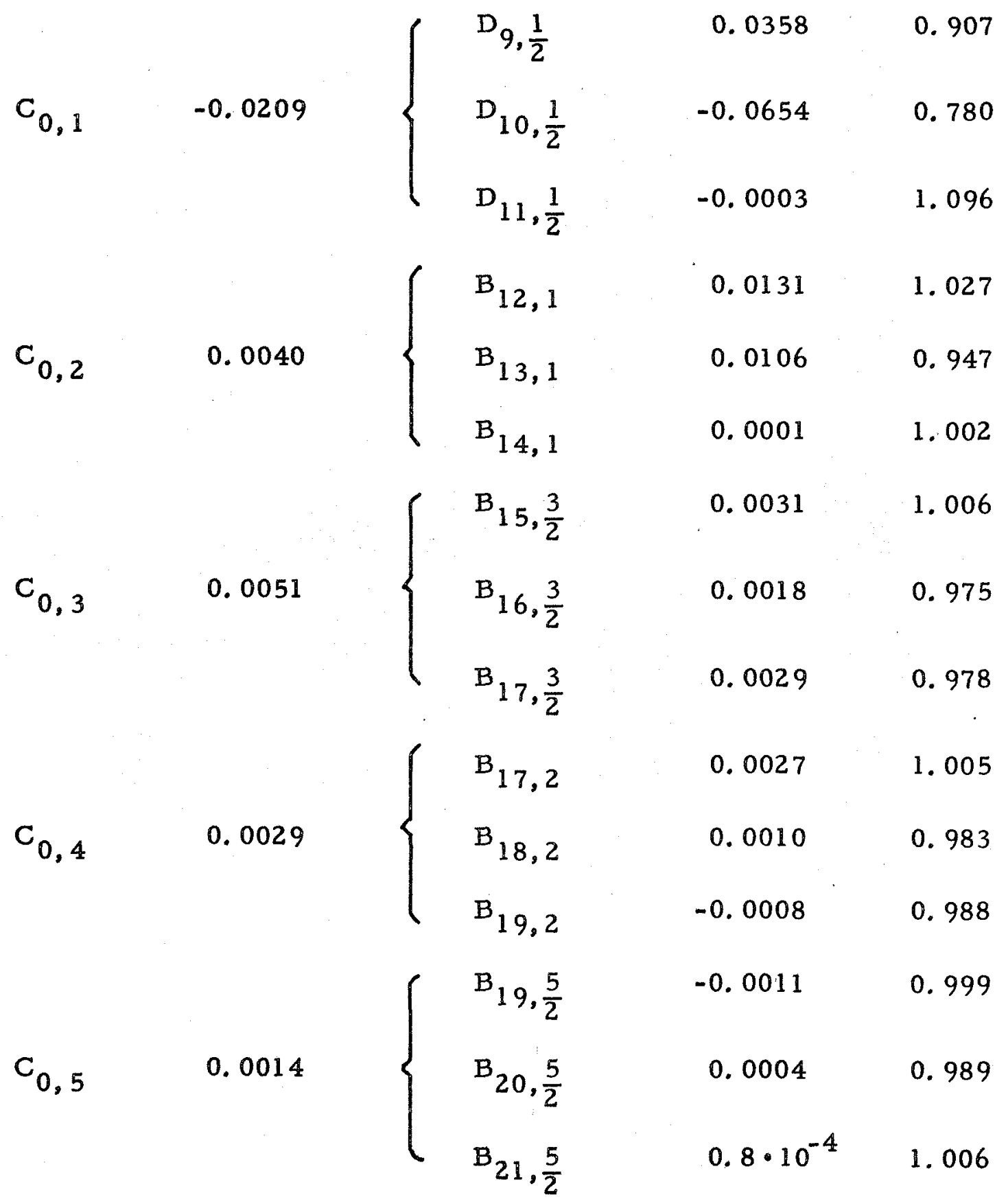


TABLE VII (Cont'd)

NUMERICAL RESULTS (Shell Al0)

\begin{tabular}{|c|c|c|c|c|}
\hline & \multicolumn{2}{|l|}{$\overline{\xi_{1}}$} & $\bar{\xi}_{2}$ or $\bar{\xi}_{3}$ & $\lambda_{M}$ \\
\hline \multirow{3}{*}{$\mathrm{C}_{0,6}$} & \multirow{3}{*}{0.0005} & $\mathrm{~B}_{21,3}$ & 0.0003 & 0.996 \\
\hline & & $B_{22,3}$ & 0.0002 & 0.995 \\
\hline & & $\mathrm{B}_{23,3}$ & -0.0013 & 0.999 \\
\hline \multirow{3}{*}{$\mathrm{C}_{0,7}$} & \multirow{3}{*}{$0.410^{-7}$} & $\mathrm{~B}_{22, \frac{7}{2}}$ & -0.0002 & 0.999 \\
\hline & & $\mathrm{B}_{23, \frac{7}{2}}$ & 0.0006 & 0.988 \\
\hline & & $\mathrm{B}_{24, \frac{7}{2}}$ & $0.3 \cdot 10^{-8}$ & 1.013 \\
\hline
\end{tabular}


143

TABLE VII (Cont'd)

NUMERICAL RESULTS (Shell A12)

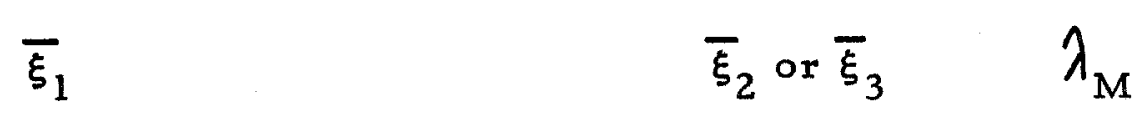

$\mathrm{A}_{0,1} \quad-0.0178$

$\mathrm{A}_{9, \frac{1}{2}}$

$-0.0164$

0.928

$\mathrm{A}_{10, \frac{1}{2}}$

$-0.0511$

0.825

$\mathrm{A}_{11, \frac{1}{2}}$

$-0.0096$

1.001

$\mathrm{A}_{0,2}$

0.0087

$\mathrm{C}_{12,1}$

0.0201

0.955

$\mathrm{C}_{13,1}$

0.0076

0.973

$\mathrm{C}_{14,1}$

$-0.0039$

0.989

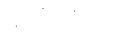

$\mathrm{A}_{0,3}$

0.0059

$C_{15, \frac{3}{2}}$

$-0.0129$

0.938

$C_{16, \frac{3}{2}}$
$C_{17, \frac{3}{2}}$

0.00004

0.987

$\mathrm{A}_{17,2}$

0.0022

0.972

$\mathrm{A}_{0,4}$

$-0.0115$

$\mathrm{A}_{18,2}$

0.0051

0.940

$A_{19,2} \quad-0.0006 \quad 0.994$

$A_{0,5} 0.0099\left\{\begin{array}{lcc}C_{19, \frac{5}{2}} & 0.0004 & 0.975 \\ C_{20, \frac{5}{2}} & 0.0041 & 0.976 \\ C_{21, \frac{5}{2}} & -0.0020 & 0.982\end{array}\right.$ 
144

TABLE VII (Cont'd)

NUMERICAL RESULTS (Shell A12)

\begin{tabular}{|c|c|c|c|c|}
\hline \multicolumn{2}{|r|}{$\bar{\xi}_{1}$} & & $\bar{\xi}_{2}$ or $\bar{\xi}_{3}$ & $\lambda_{\mathrm{M}}$ \\
\hline \multirow{3}{*}{$A_{0,6}$} & \multirow{3}{*}{-0.0075} & $A_{21,3}$ & -0.0012 & 0.962 \\
\hline & & $\mathrm{A}_{22,3}$ & -0.0011 & 0.972 \\
\hline & & $A_{23,3}$ & 0.0007 & 1.008 \\
\hline \multirow{3}{*}{$A_{0,7}$} & \multirow{3}{*}{-0.0024} & $\mathrm{~A}_{22}, \frac{7}{2}$ & .0 .0010 & 0.975 \\
\hline & & $\mathrm{A}_{23, \frac{7}{2}}$ & -0.0001 & 0.992 \\
\hline & & $\mathrm{A}_{24, \frac{7}{2}}$ & 0.0010 & 1.002 \\
\hline
\end{tabular}


145

TABLE VII (Cont'd)

NUMERICAL RESULTS (Shell A12)

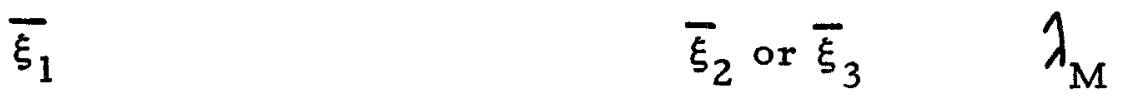

$A_{0,1}-0.0178\left\{\begin{array}{lcl}B_{10, \frac{1}{2}} & -0.0117 & 0.947 \\ B_{11, \frac{1}{2}} & 0.0601 & 0.910\end{array}\right.$

\begin{tabular}{|c|c|c|c|c|}
\hline & & $\mathrm{D}_{12,1}$ & 0.0118 & 0.981 \\
\hline \multirow[t]{3}{*}{$\mathrm{A}_{0,2}$} & 0.0087 & $\mathrm{D}_{13,1}$ & -0.0057 & 0.982 \\
\hline & & $\mathrm{D}_{14,1}$ & 0.0082 & 0.967 \\
\hline & & $\mathrm{D}_{15, \frac{3}{2}}$ & -0.0120 & 0.941 \\
\hline \multirow[t]{3}{*}{$A_{0,3}$} & 0.0059 & $D_{16, \frac{3}{2}}$ & 0.0019 & 0.968 \\
\hline & & $D_{17, \frac{3}{2}}$ & -0.0009 & 1.018 \\
\hline & & $\mathrm{B}_{17,2}$ & 0.0038 & 0.963 \\
\hline \multirow[t]{3}{*}{$\mathrm{A}_{0,4}$} & -0.0115 & $\mathrm{~B}_{18,2}$ & -0.0003 & 0.975 \\
\hline & & $\mathrm{B}_{19,2}$ & -0.0028 & 0.975 \\
\hline & & $\mathrm{D}_{19, \frac{5}{2}}$ & -0.0006 & 0.973 \\
\hline \multirow[t]{2}{*}{$\mathrm{A}_{0,5}$} & 0.0099 & $D_{20, \frac{5}{2}}$ & -0.0005 & 1.006 \\
\hline & & $\mathrm{D}_{21, \frac{5}{2}}$ & 0.0037 & 0.969 \\
\hline
\end{tabular}


TABLE VII (Cont'd)

NUMERICAL RESULTS (Shell A12)

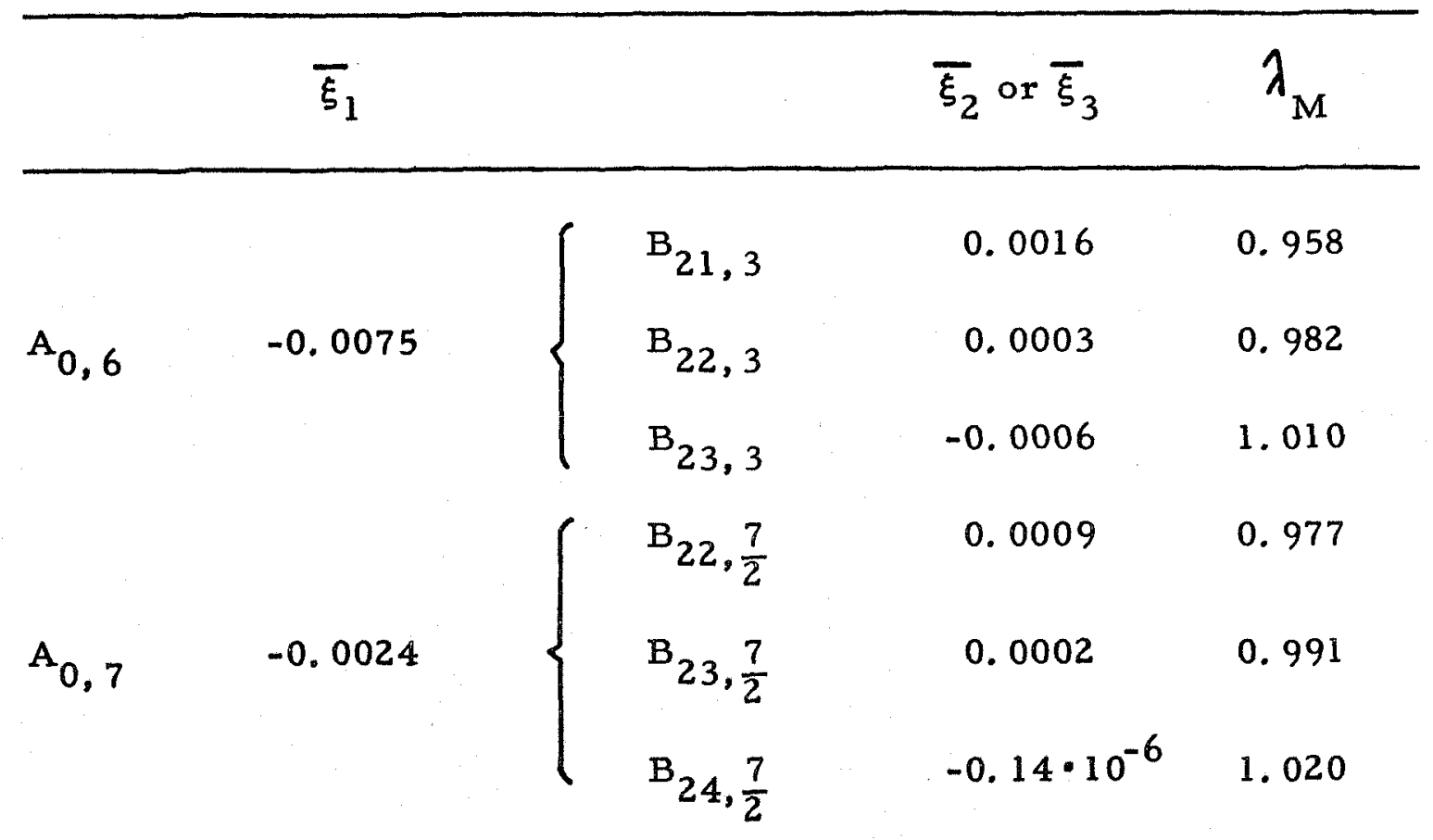


TABLE VII (Cont'd)

NUMERICAL RESULTS (Shell A12)

\begin{tabular}{|c|c|c|c|c|}
\hline \multicolumn{2}{|r|}{$\bar{\xi}_{1}$} & \multicolumn{2}{|r|}{$\bar{\xi}_{2}$ or $\bar{\xi}_{3}$} & $\lambda_{M}$ \\
\hline \multirow{3}{*}{$C_{0,1}$} & \multirow{3}{*}{0.0092} & $A_{9, \frac{1}{2}}$ & -0.0164 & 0.931 \\
\hline & & $A_{10, \frac{1}{2}}$ & -0.0511 & 0.828 \\
\hline & & $A_{11, \frac{1}{2}}$ & -0.0096 & 1.105 \\
\hline \multirow{3}{*}{$\mathrm{C}_{0,2}$} & \multirow{3}{*}{0.0280} & $\mathrm{~A}_{12,1}$ & -0.0151 & 0.955 \\
\hline & & $\mathrm{A}_{13,1}$ & -0.0057 & 0.937 \\
\hline & & $\mathrm{A}_{14,1}$ & -0.0023 & 0.984 \\
\hline \multirow{3}{*}{$C_{0,3}$} & \multirow{3}{*}{-0.0107} & $C_{15, \frac{3}{2}}$ & -0.0129 & 0.932 \\
\hline & & $C_{16, \frac{3}{2}}$ & 0.00004 & 0.987 \\
\hline & & $C_{17, \frac{3}{2}}$ & 0.0007 & 1.015 \\
\hline \multirow{3}{*}{$C_{0,4}$} & \multirow{3}{*}{0.0092} & $\mathrm{~A}_{17,2}$ & 0.0022 & 0.976 \\
\hline & & $\mathrm{A}_{18,2}$ & 0.0051 & 0.944 \\
\hline & & $\mathrm{A}_{19,2}$ & -0.0006 & 0.998 \\
\hline \multirow{3}{*}{$C_{0,5}$} & \multirow{3}{*}{0.0050} & $A_{19, \frac{5}{2}}$ & -0.0003 & 0.987 \\
\hline & & $\mathrm{A}_{20, \frac{5}{2}}$ & 0.0014 & 0.973 \\
\hline & & $A_{21, \frac{5}{2}}$ & 0.0017 & 0.995 \\
\hline
\end{tabular}


148

TABLE VII (Cont'd)

NUMERICAL RESULTS (Shell A12)

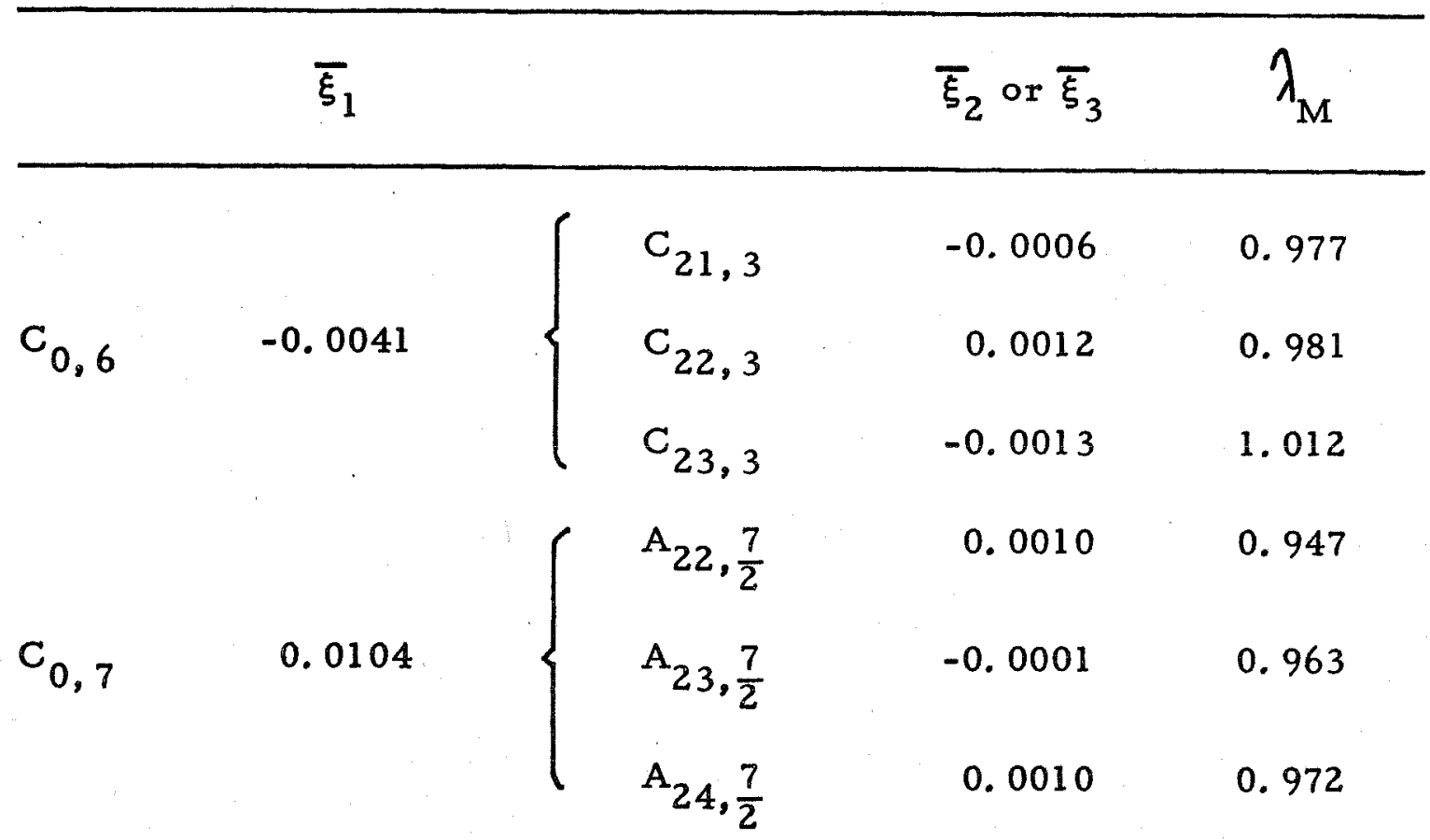


TABLE VII (Cont'd)

NUMERICAL RESULTS (Shell A12)

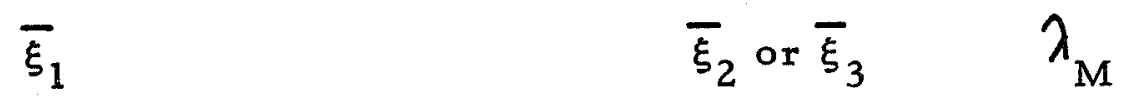

\begin{tabular}{|c|c|c|c|c|}
\hline & & $B_{9}, \frac{1}{2}$ & -0.0887 & 0.767 \\
\hline \multirow[t]{3}{*}{$\mathrm{C}_{0,1}$} & 0.0092 & $B_{10, \frac{1}{2}}$ & -0.0117 & 0.950 \\
\hline & & $B_{11, \frac{1}{2}}$ & 0.0601 & 0.913 \\
\hline & & $\mathrm{B}_{12,1}$ & -0.0076 & 0.982 \\
\hline \multirow[t]{3}{*}{$c_{0,2}$} & 0.0280 & $\mathrm{~B}_{13,1}$ & 0.0280 & 0.861 \\
\hline & & $\mathrm{B}_{14,1}$ & -0.0087 & 0.949 \\
\hline & & $D_{15, \frac{3}{2}}$ & -0.0120 & 0.936 \\
\hline \multirow[t]{3}{*}{$\mathrm{C}_{0,3}$} & -0.0107 & $D_{16, \frac{3}{2}}$ & 0.0019 & 0.968 \\
\hline & & $D_{17, \frac{3}{2}}$ & -0.0009 & 1.012 \\
\hline & & $B_{17,2}$ & 0.0038 & 0.966 \\
\hline \multirow[t]{3}{*}{$C_{0,4}$} & 0.0092 & $B_{18,2}$ & -0.0003 & 0.979 \\
\hline & & $B_{19,2}$ & -0.0028 & 0.979 \\
\hline & & $B_{19, \frac{5}{2}}$ & 0.0018 & 0.973 \\
\hline \multirow[t]{2}{*}{$C_{0,5}$} & 0.0050 & $\mathrm{~B}_{20, \frac{5}{2}}$ & 0.0015 & 0.972 \\
\hline & & & 0.0034 & 0.982 \\
\hline
\end{tabular}


TABLE VII (Cont'd)

NUMERICAL RESULTS (Shell A12)

\begin{tabular}{llll}
\hline $\bar{\xi}_{1}$ & $\bar{\xi}_{2}$ or $\bar{\xi}_{3}$ & $\lambda_{M}$ \\
$\mathrm{C}_{0,6}$ & -0.0041 & $\left\{\begin{array}{lll}\mathrm{D}_{21,3} & 0.0013 & 0.970 \\
\mathrm{D}_{22,3} & -0.0001 & 0.996 \\
\mathrm{D}_{23,3} & -0.0010 & 1.014 \\
\mathrm{~B}_{22, \frac{7}{2}} & 0.0009 & 0.949 \\
\mathrm{~B}_{23, \frac{7}{2}} & 0.0002 & 0.961 \\
\mathrm{~B}_{24, \frac{7}{2}} & -0.1410^{-6} & 0.989\end{array}\right.$
\end{tabular}


COMPARISON OF THEORY AND EXPERIMENT

\begin{tabular}{|c|c|c|c|c|c|c|}
\hline Shell & $\lambda_{\exp }$ & $\lambda_{\text {crit }}$ & \multicolumn{2}{|c|}{$\begin{array}{c}\text { Pair of Critical Modal } \\
\text { Components }\end{array}$} & $\Delta \lambda$ & Remarks \\
\hline A. 7 & $0.553 / 0.597$ & 0.794 & $c_{0,1}$ & $C_{10, \frac{1}{2}}$ & 0.241 & $\begin{array}{l}\text { Local } \\
\text { Buckling }\end{array}$ \\
\hline A8 & 0.658 & 0.651 & $C_{0,1}$ & ${ }^{B} 9, \frac{1}{2}$ & -0.007 & \\
\hline A9 & 0.736 & 0.827 & $c_{0,1}$ & $\mathrm{C}_{9, \frac{1}{2}}$ & 0.091 & \\
\hline Al 0 & $0.554 / 0.568$ & 0.780 & $\mathrm{c}_{0,1}$ & $\mathrm{D}_{10, \frac{1}{2}}$ & 0.226 & $\begin{array}{l}\text { Local } \\
\text { Buckling }\end{array}$ \\
\hline $\mathrm{A} 12$ & 0.673 & 0.764 & ${ }^{A} 0,1$ & ${ }^{B} 9, \frac{1}{2}$ & 0.091 & \\
\hline
\end{tabular}




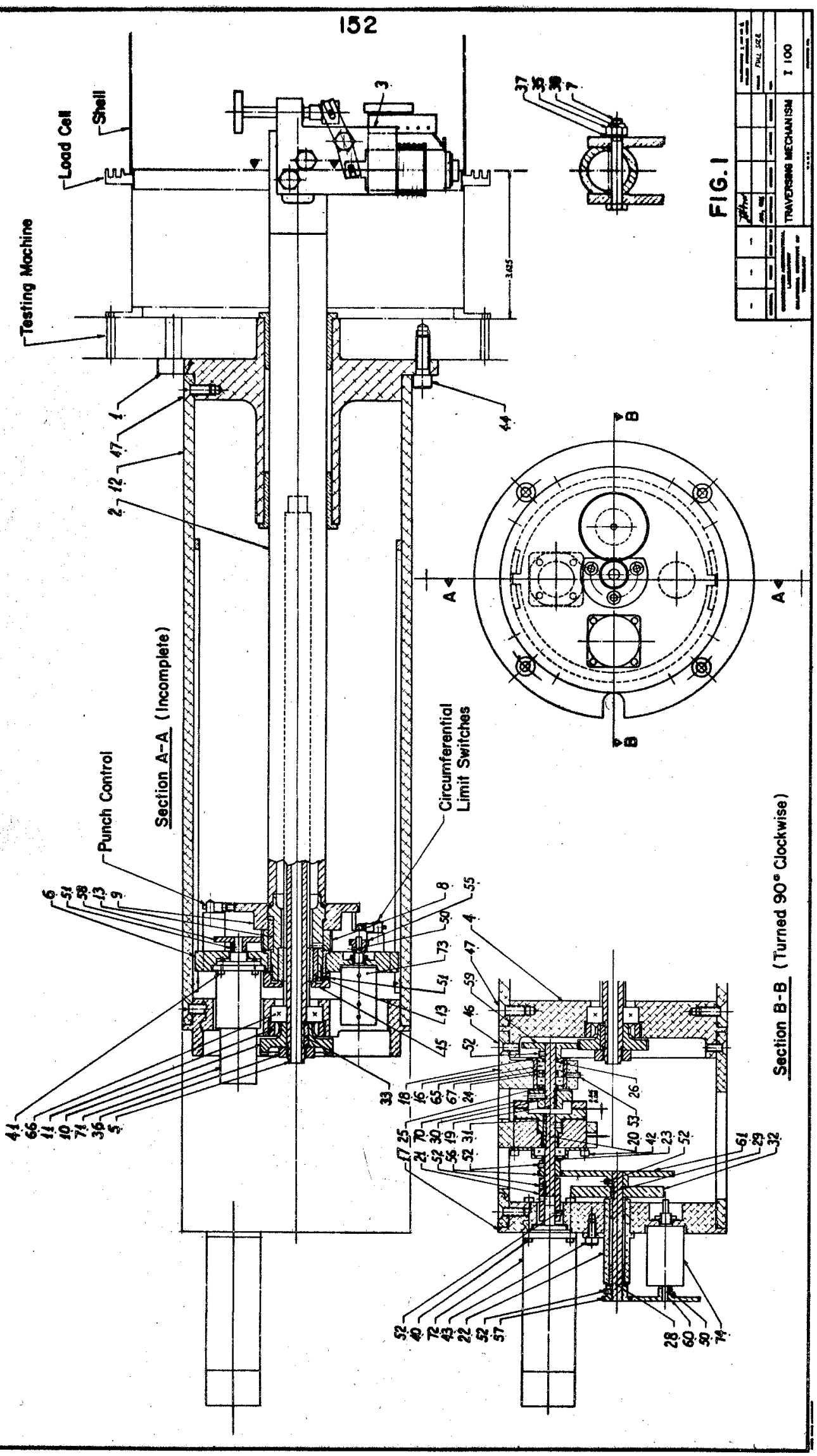


153

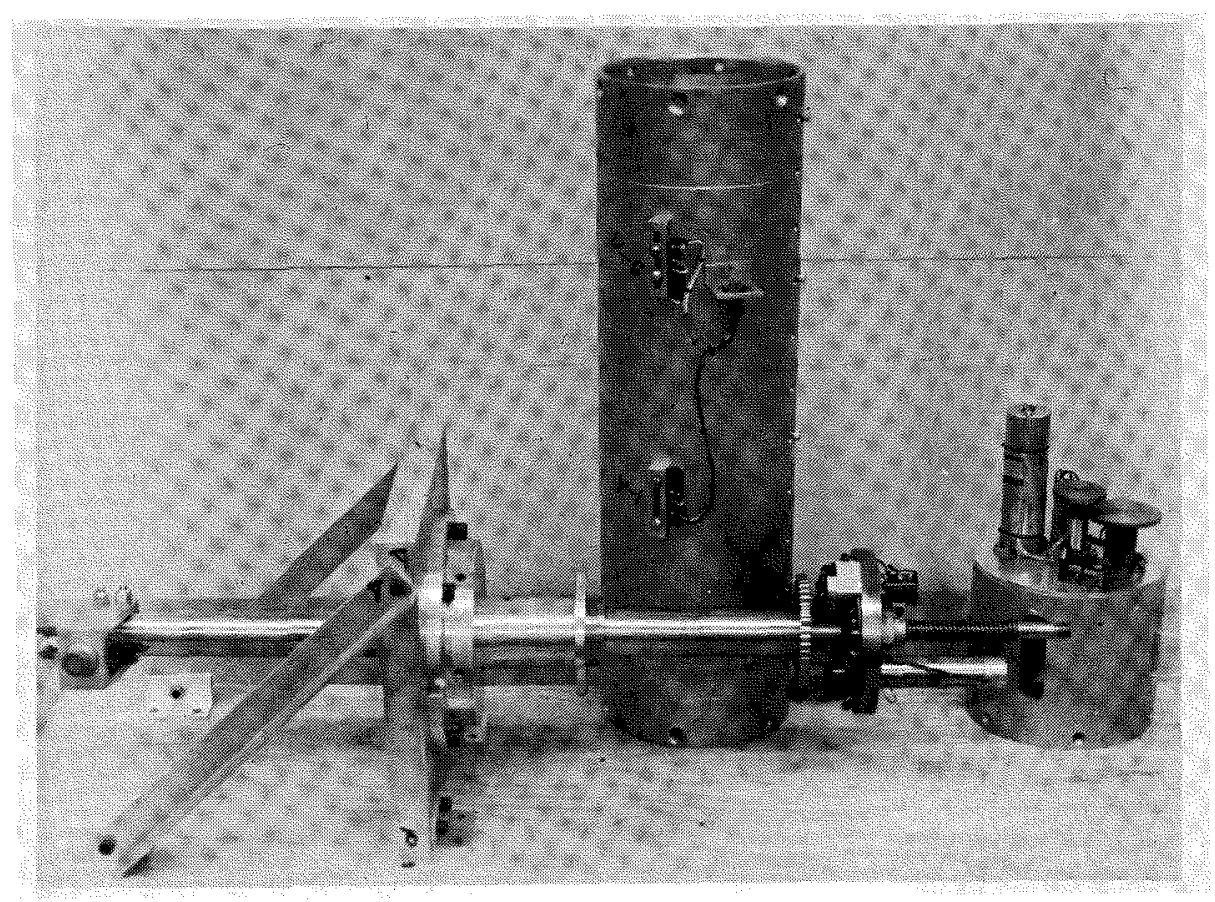

FIG. 2 PARTIALLY ASSEMBLED SCANNING MECHANISM 


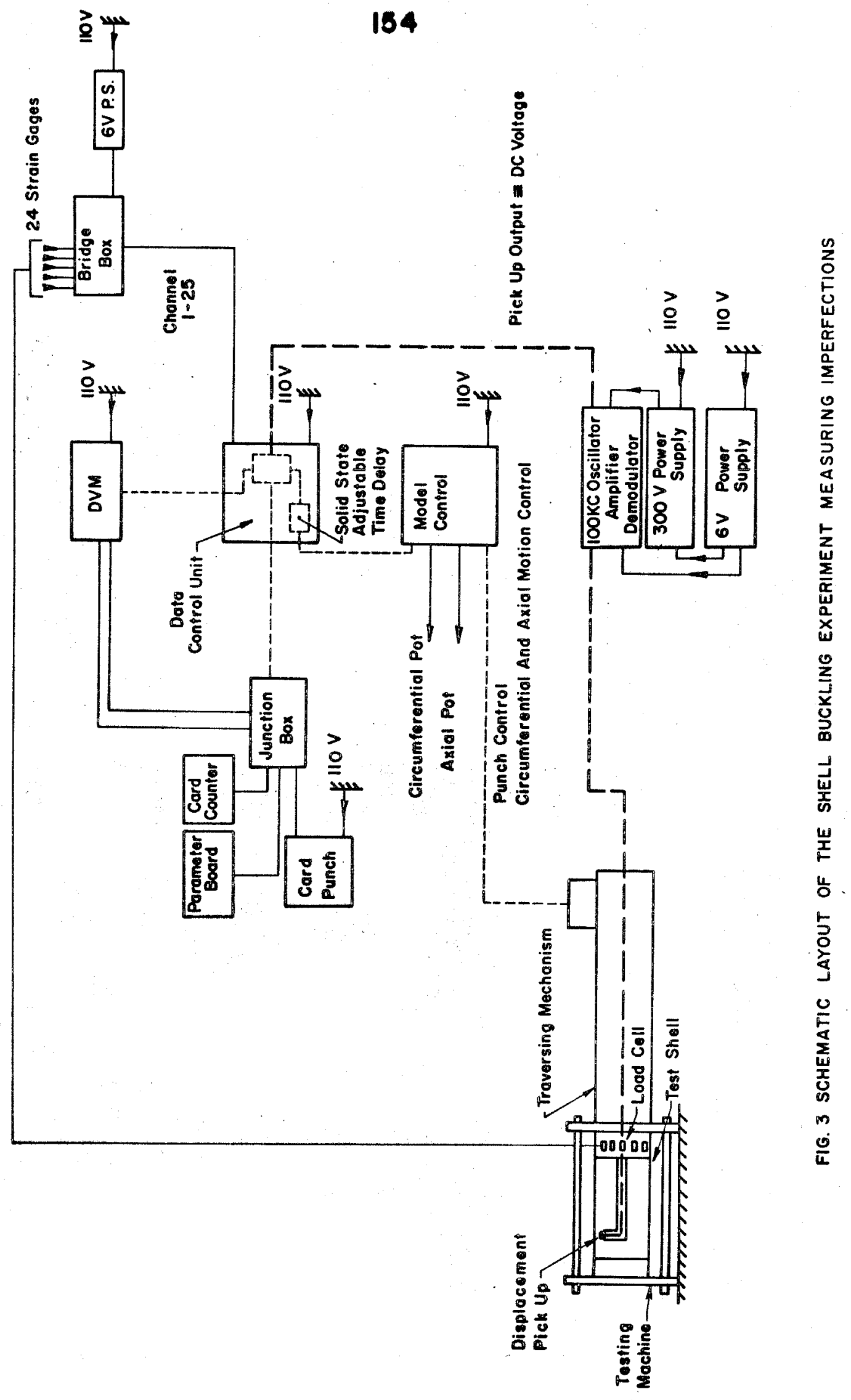




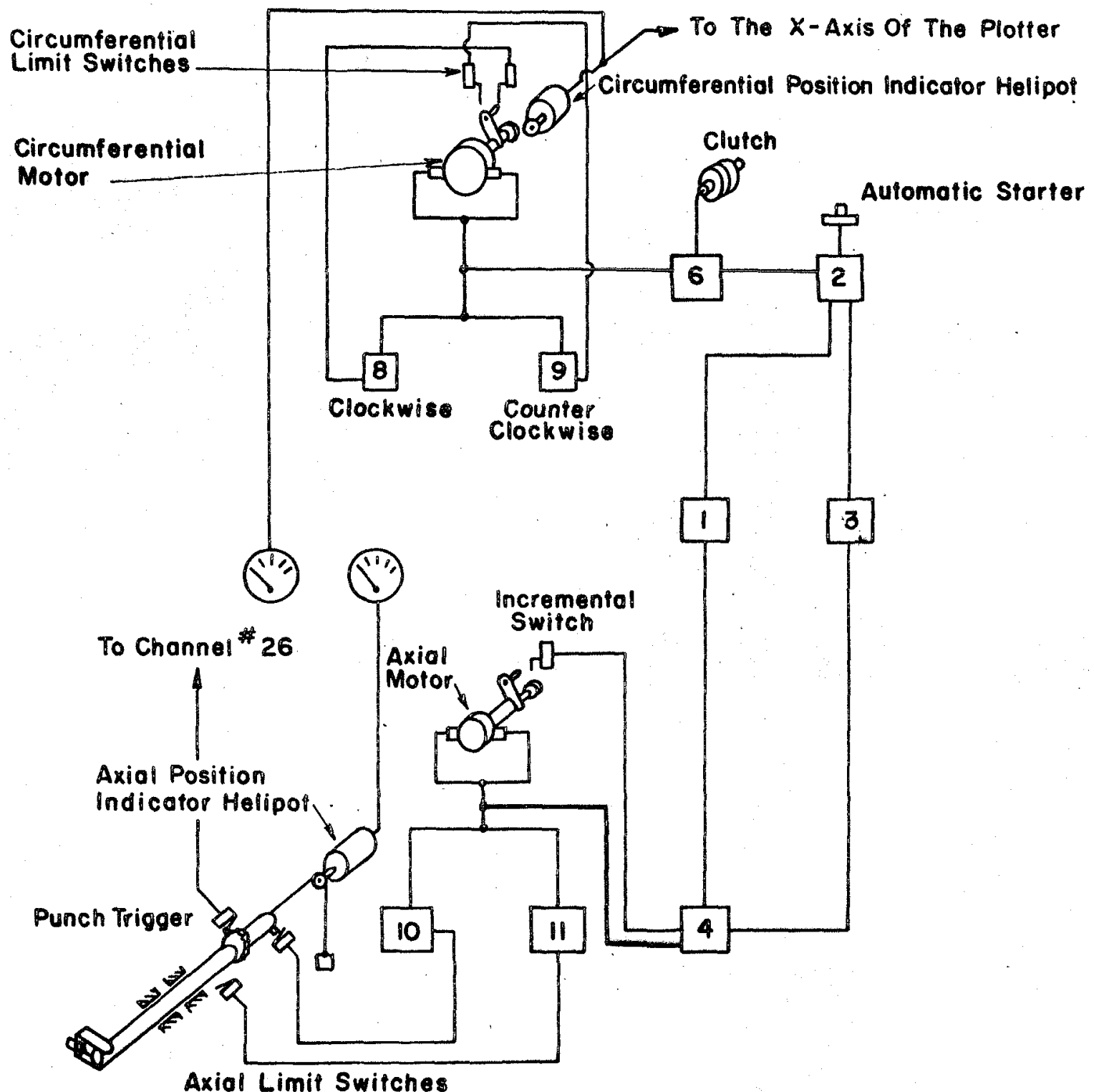

FIG. 4 MODEL CONTROL UNIT 


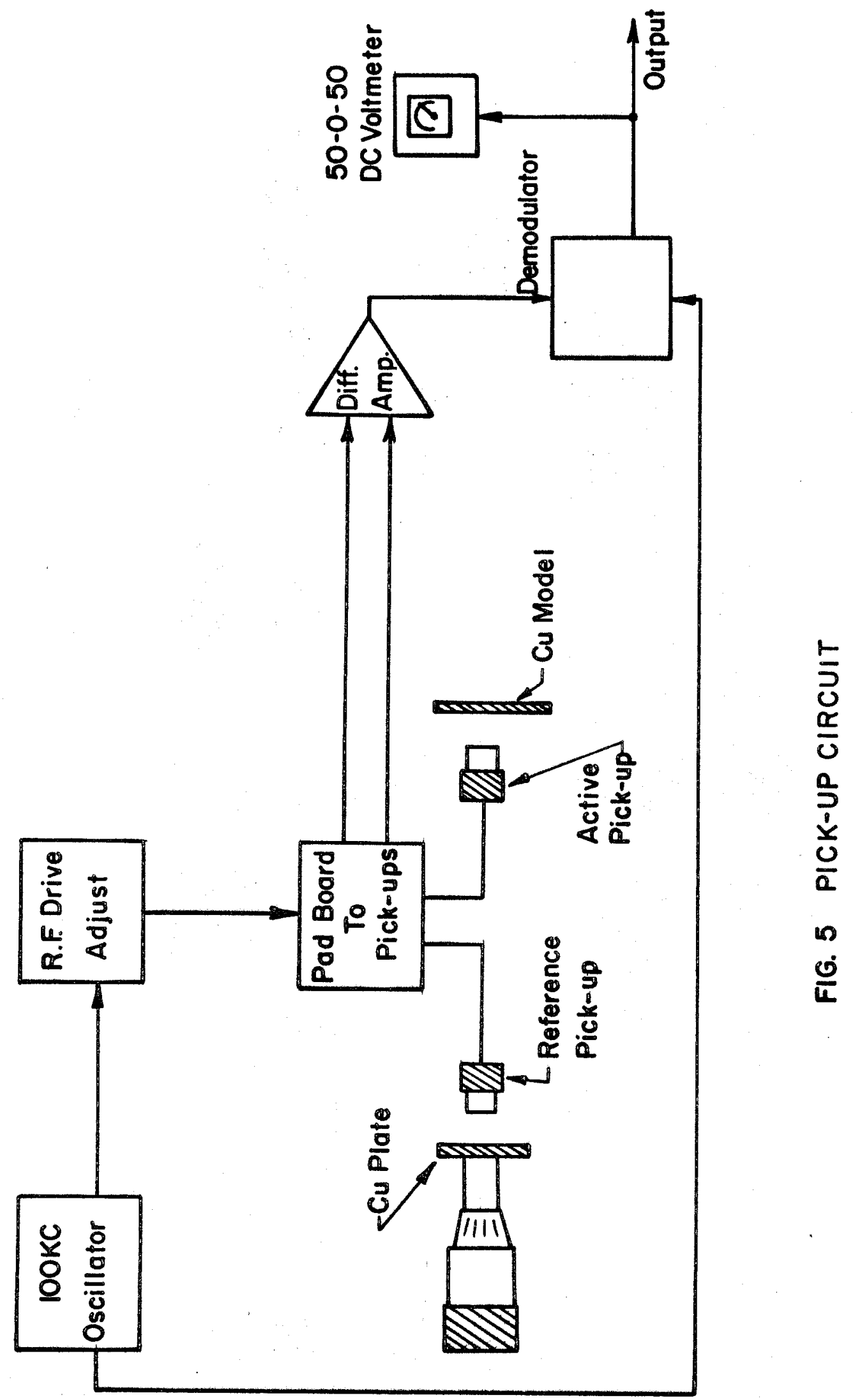




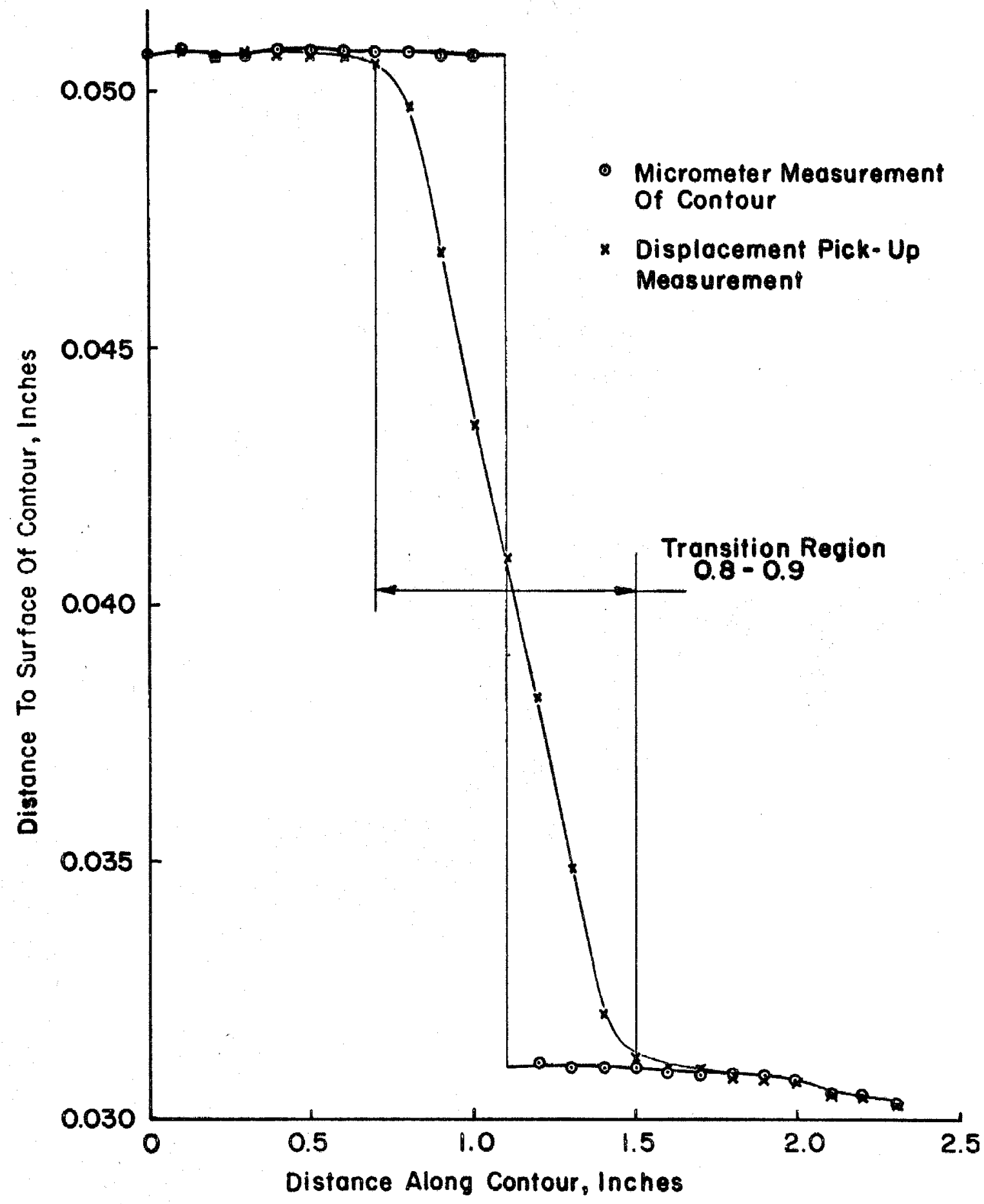

FIG. 6 MICROMETER MEASUREMENT OF KNOWN CONTOUR COMPARED TO PICK-UP MEASUREMENTS 


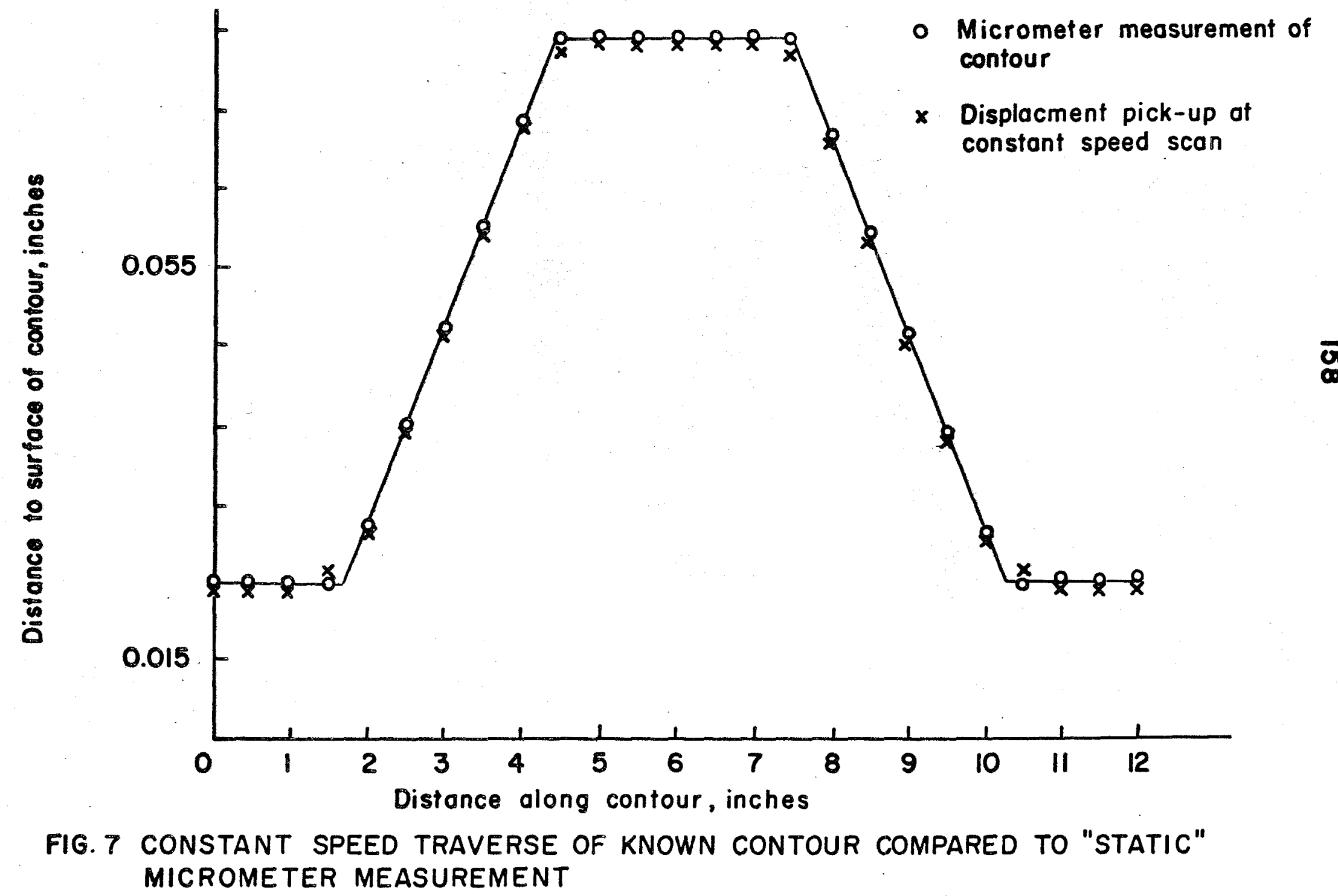

ज্ 


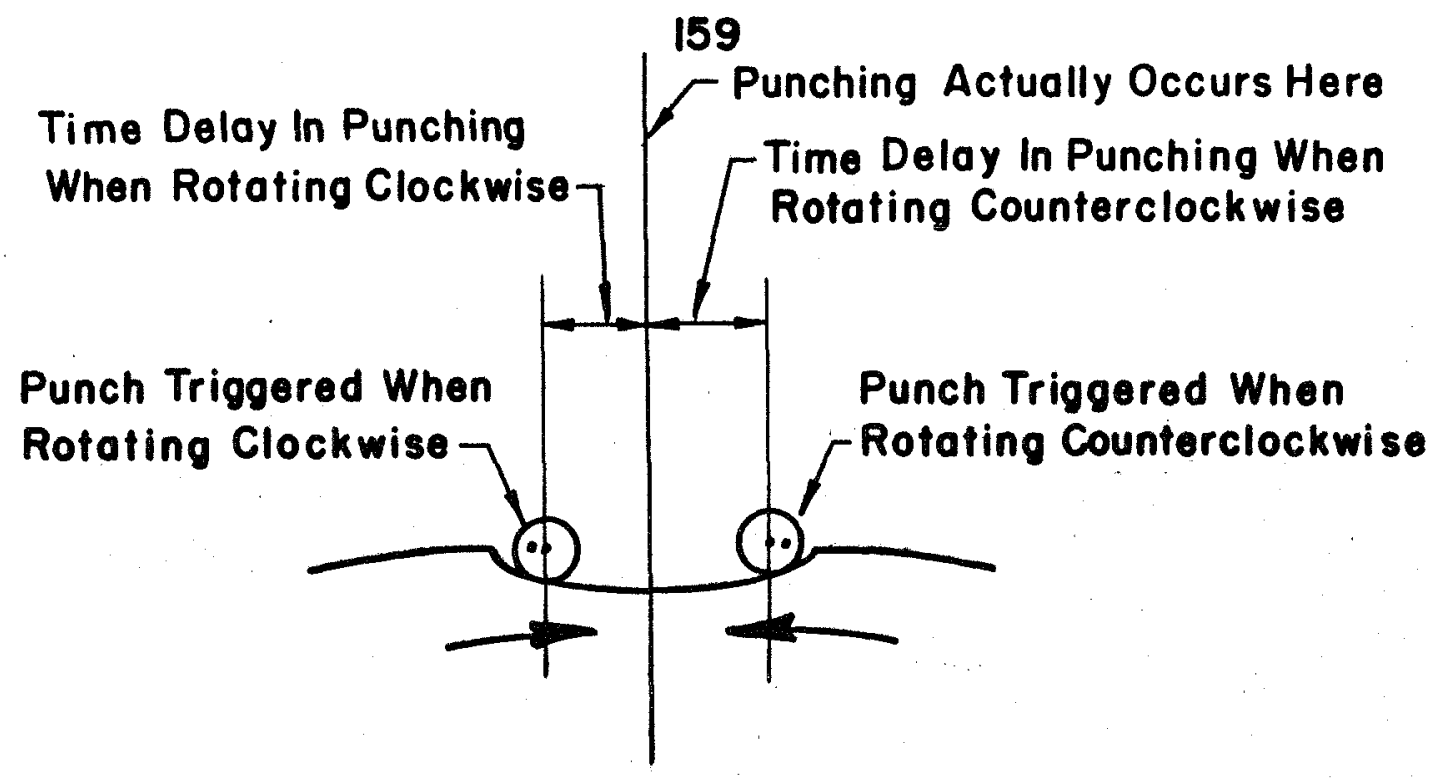

FIG.8 TIME DELAY FOR PUNCH CONTROL

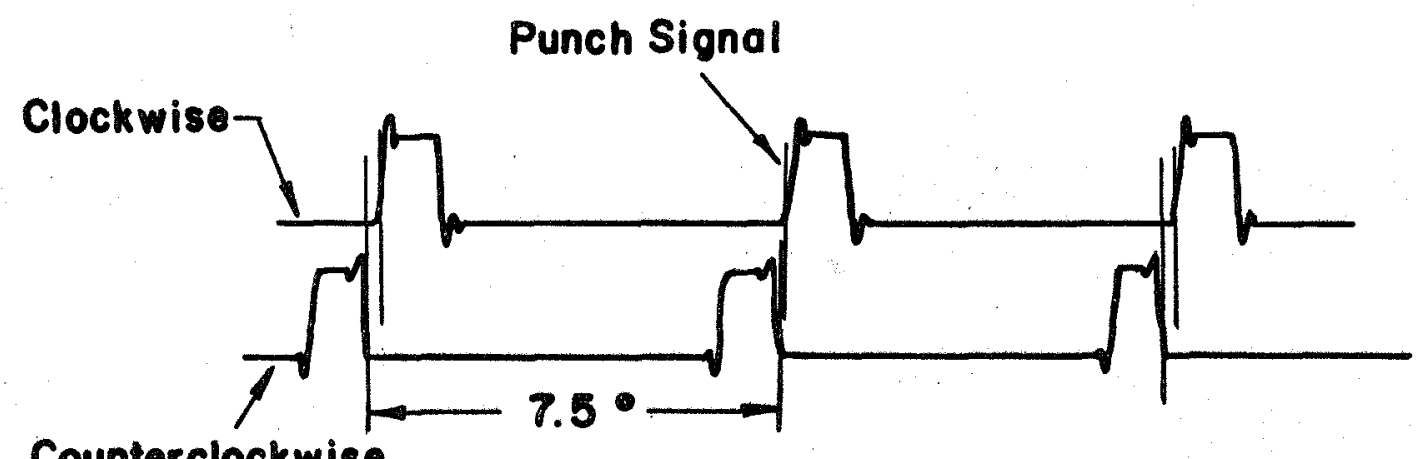

Counterclockwise 


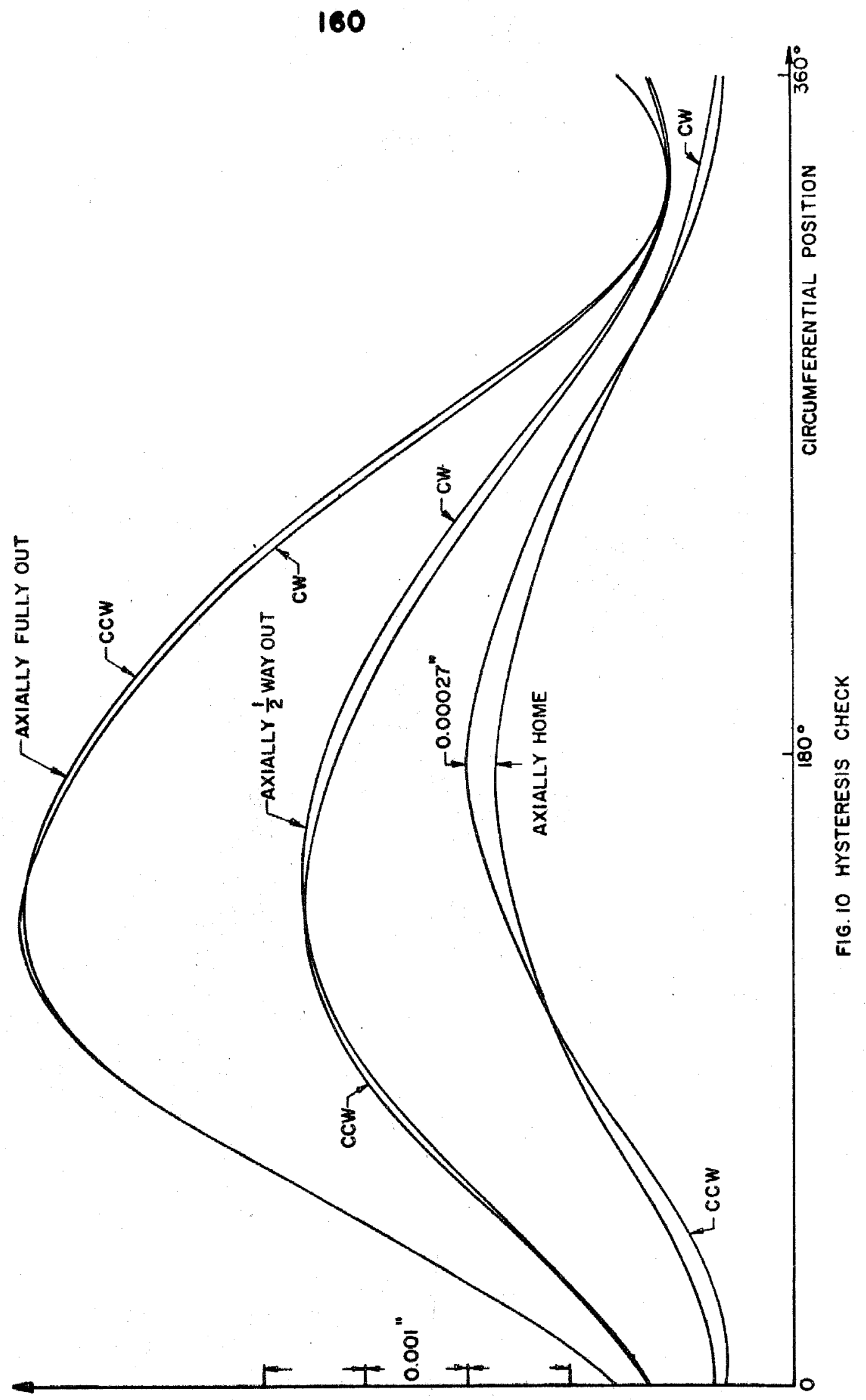


161

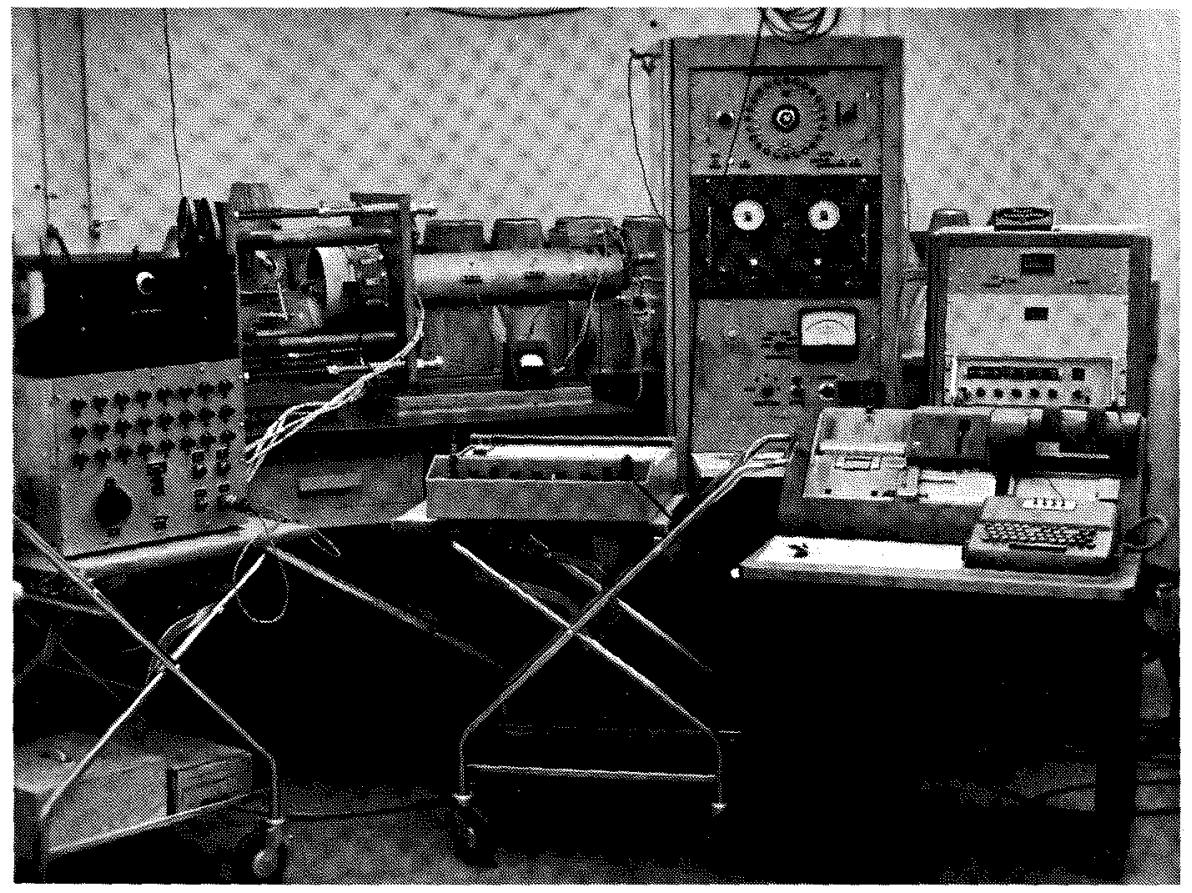

Fig.ll Testing Machine and Data Acquisition Equipment 
162

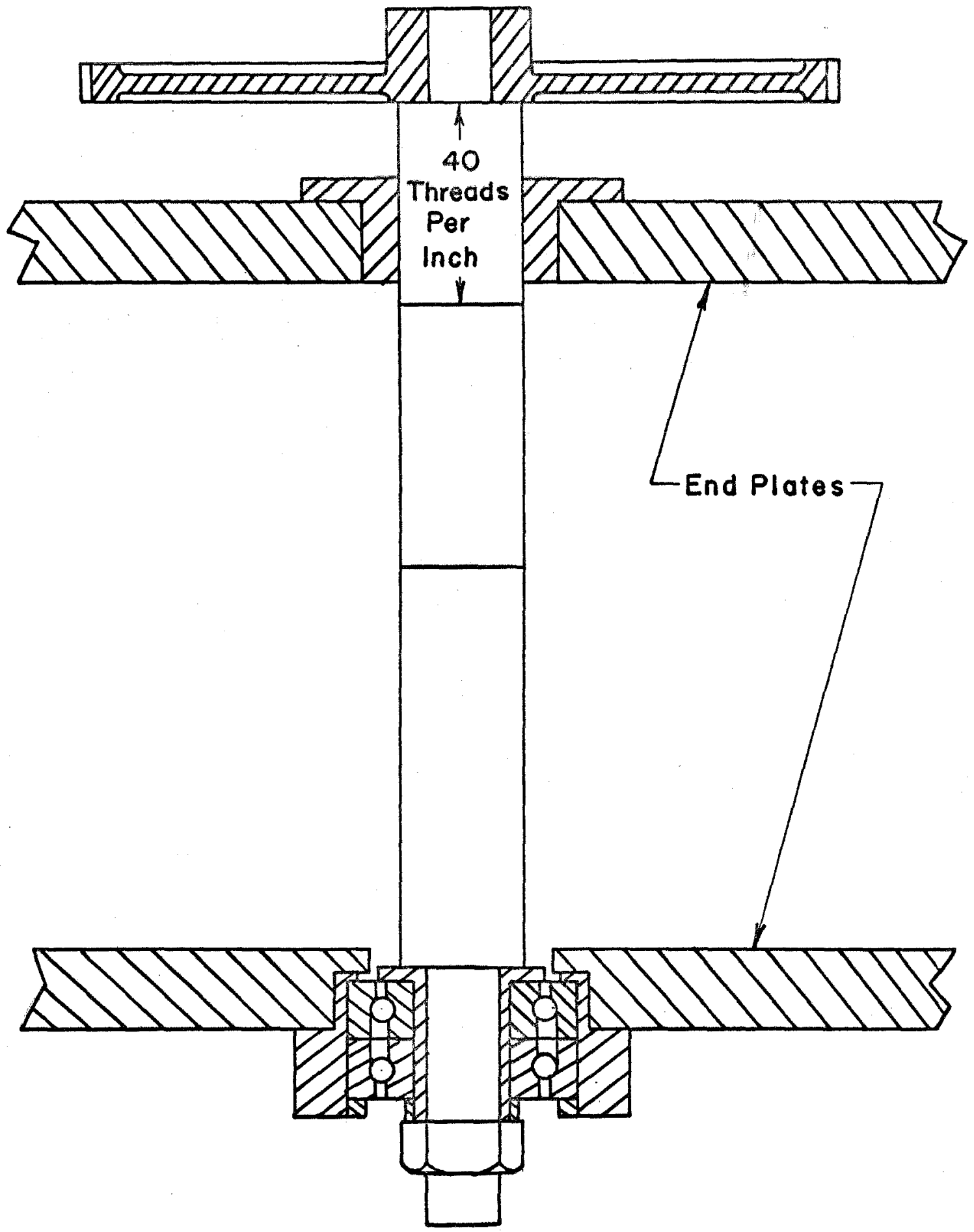

FIG.I2 DETAILS OF TESTING MACHINE LOADING SCREW 


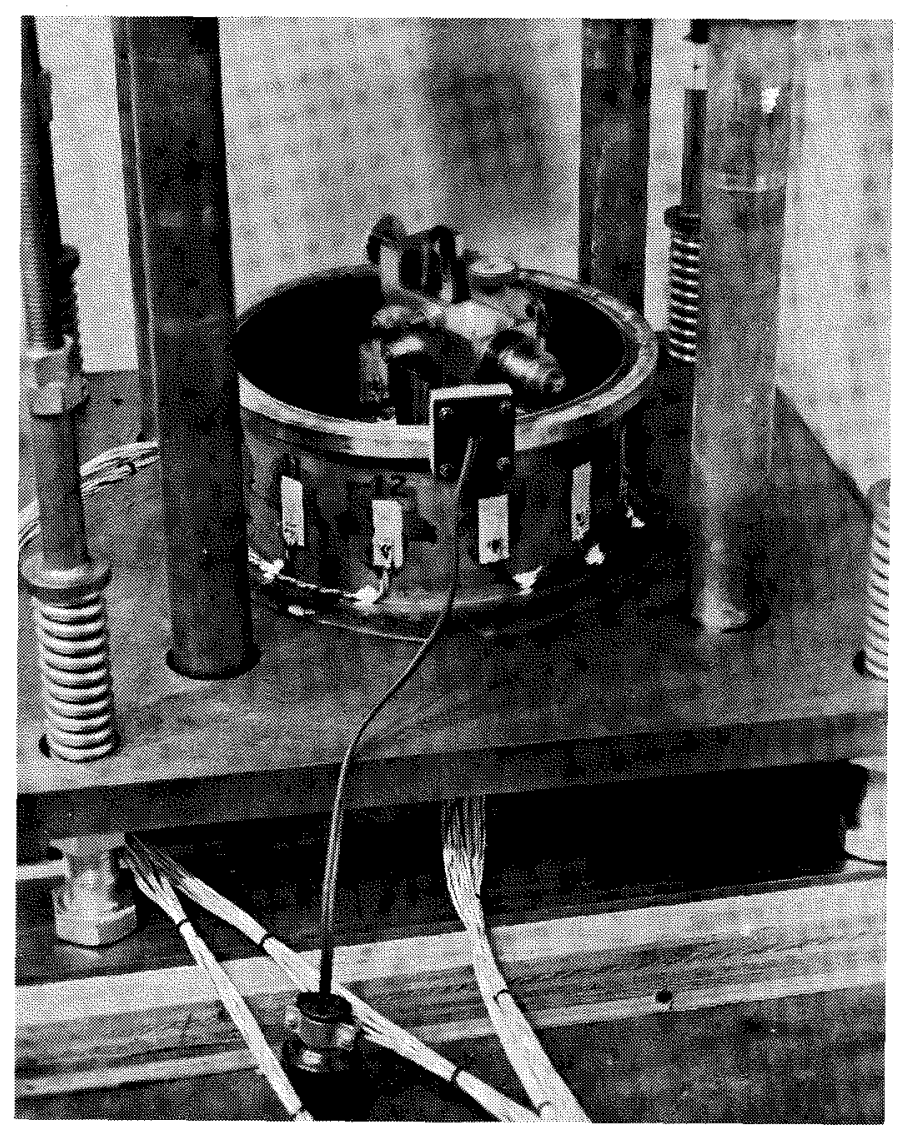

FIG. 13 Load Cell 


\section{4}

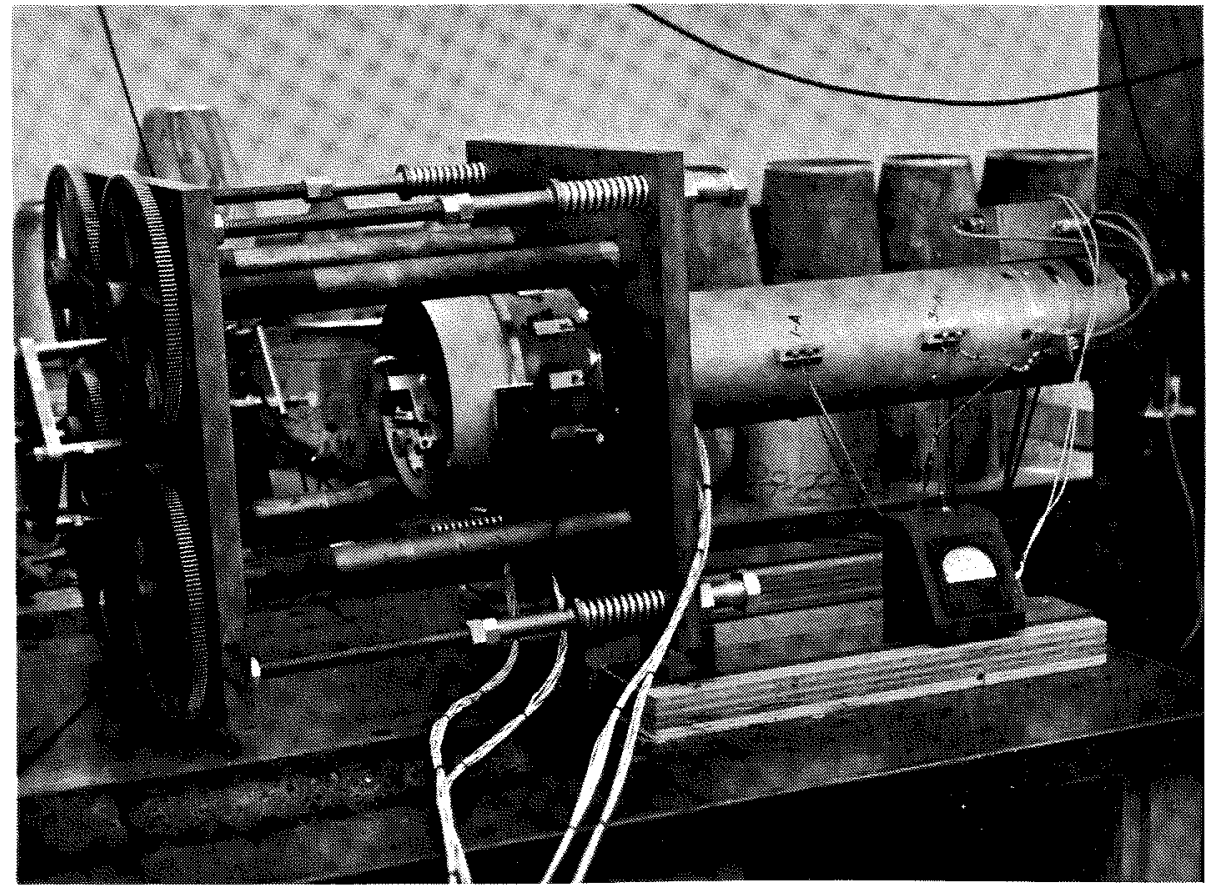

Fig. 14 Pick-up Calibration Set Up 
165

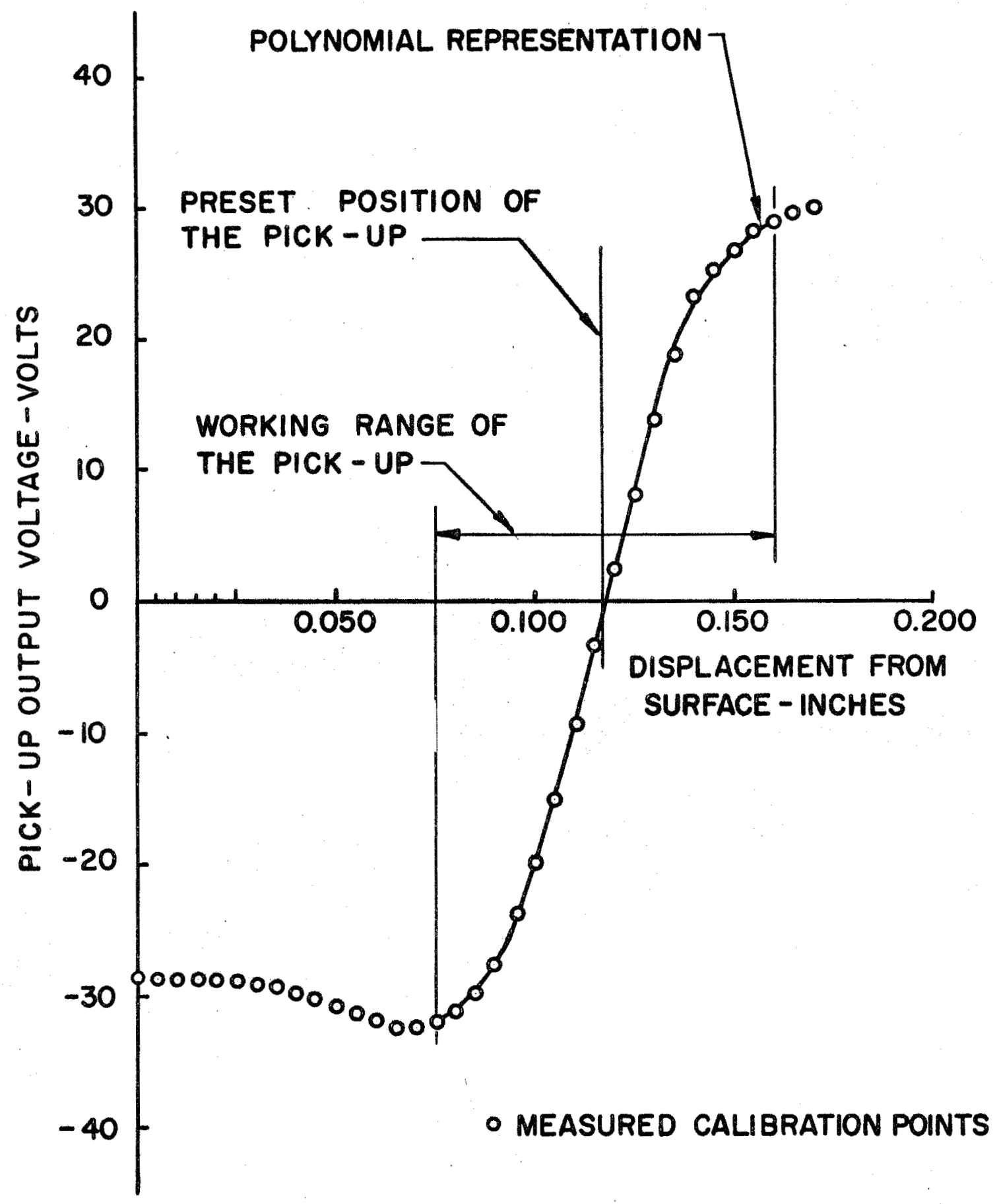

FIG. 15 TYPICAL PICK-UP CALIBRATION CURVE 
166

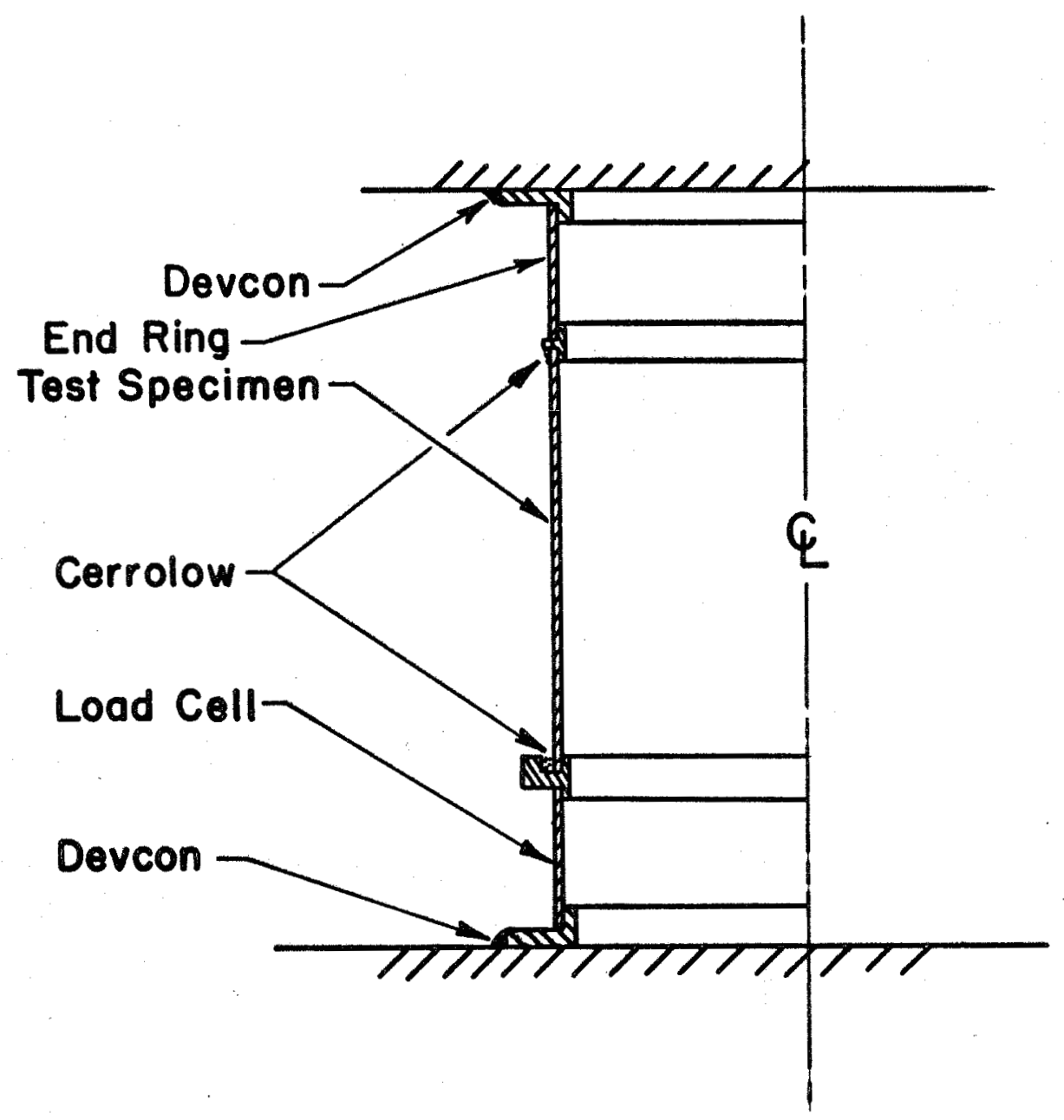

FIG. 16 CYLINDRICAL SHELL TESTING CONFIGURATION 

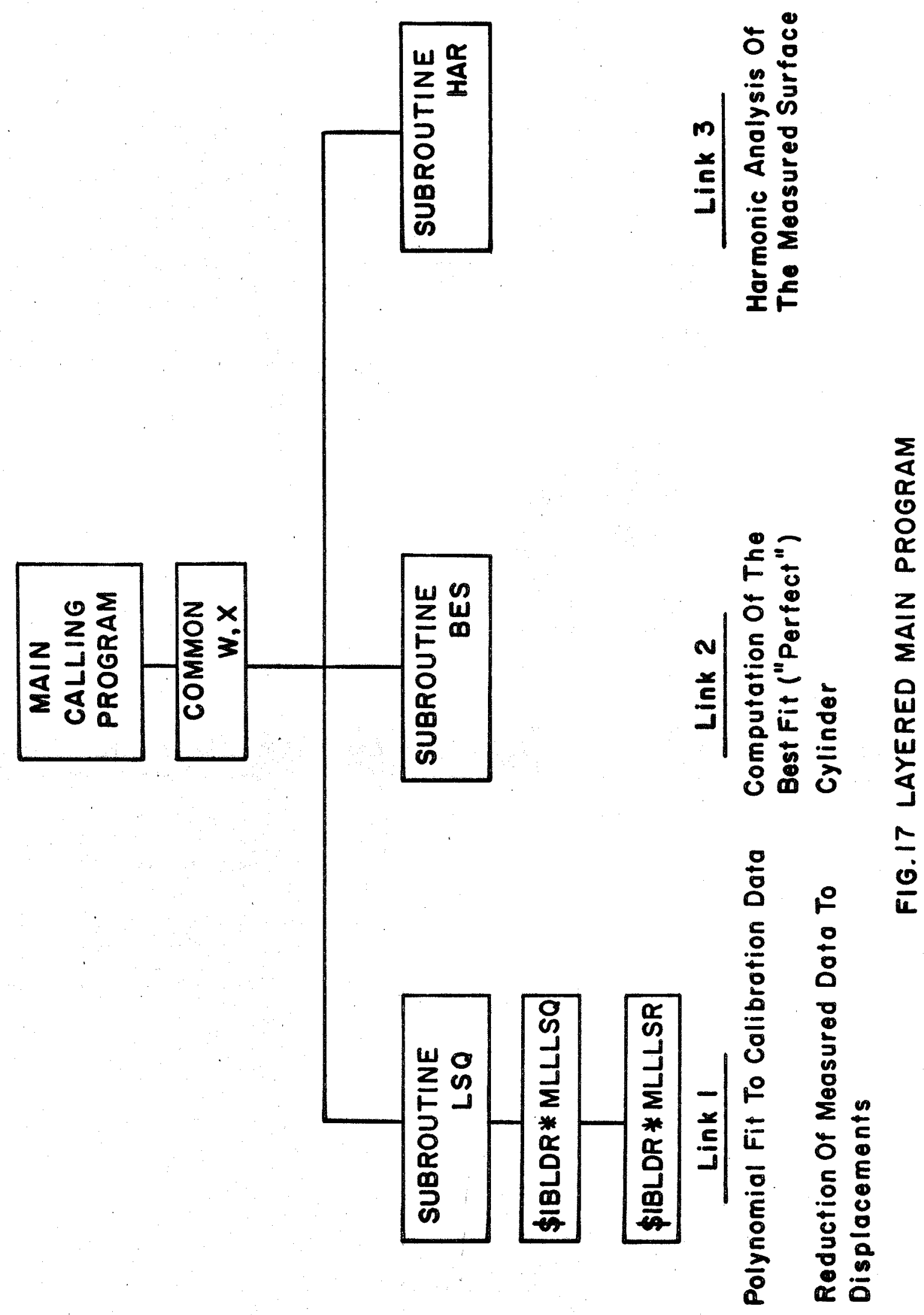


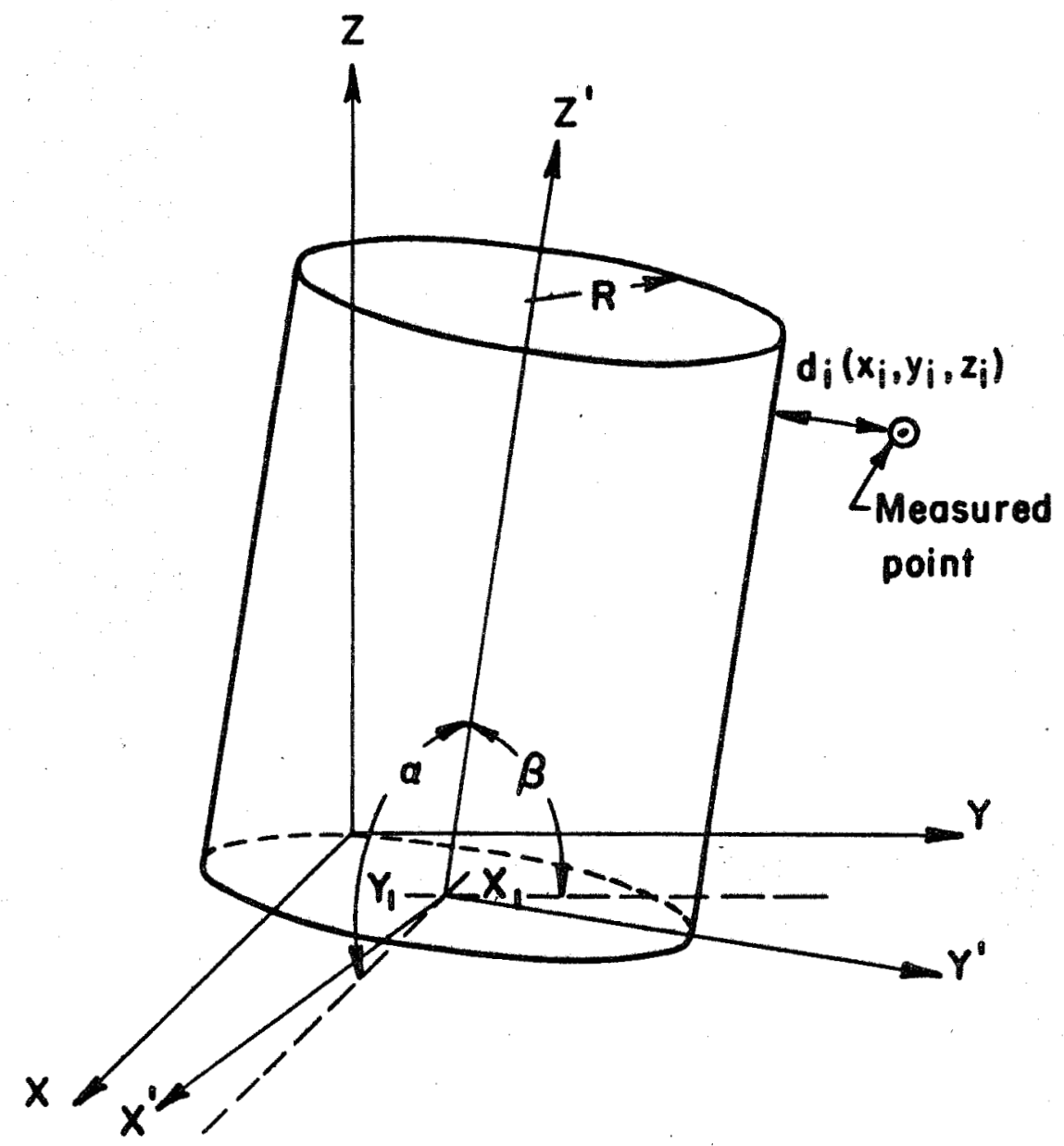

$X, Y, Z$ Reference axis of traversing pick-up $X^{\prime}, Y^{\prime}, Z^{\prime}$ Reference axis of best fit cylinder

di Normal distance from measured point to best fit cylinder

FIG. 18 BEST FIT CYLINDER REFERENCE AXIS 


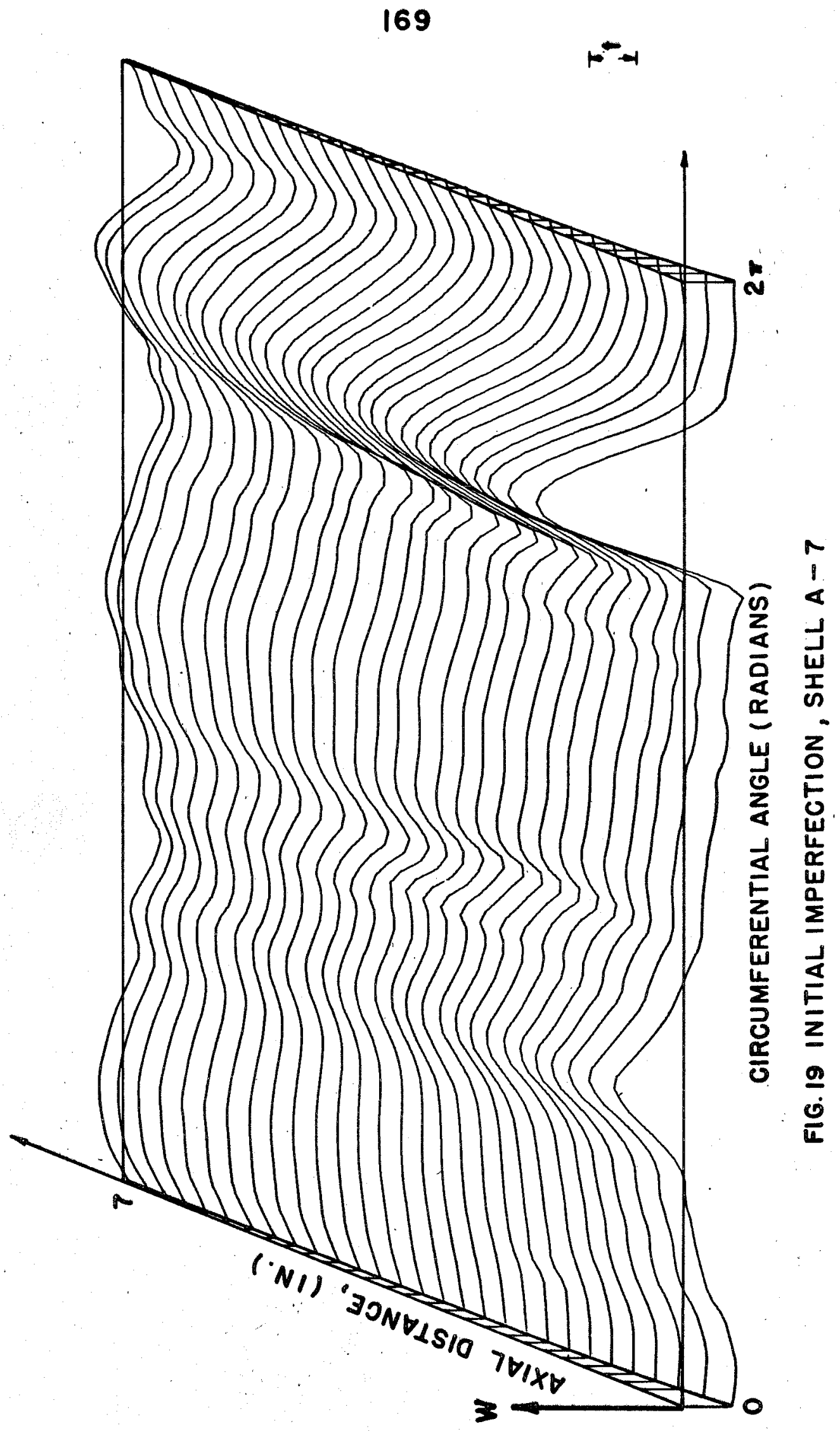




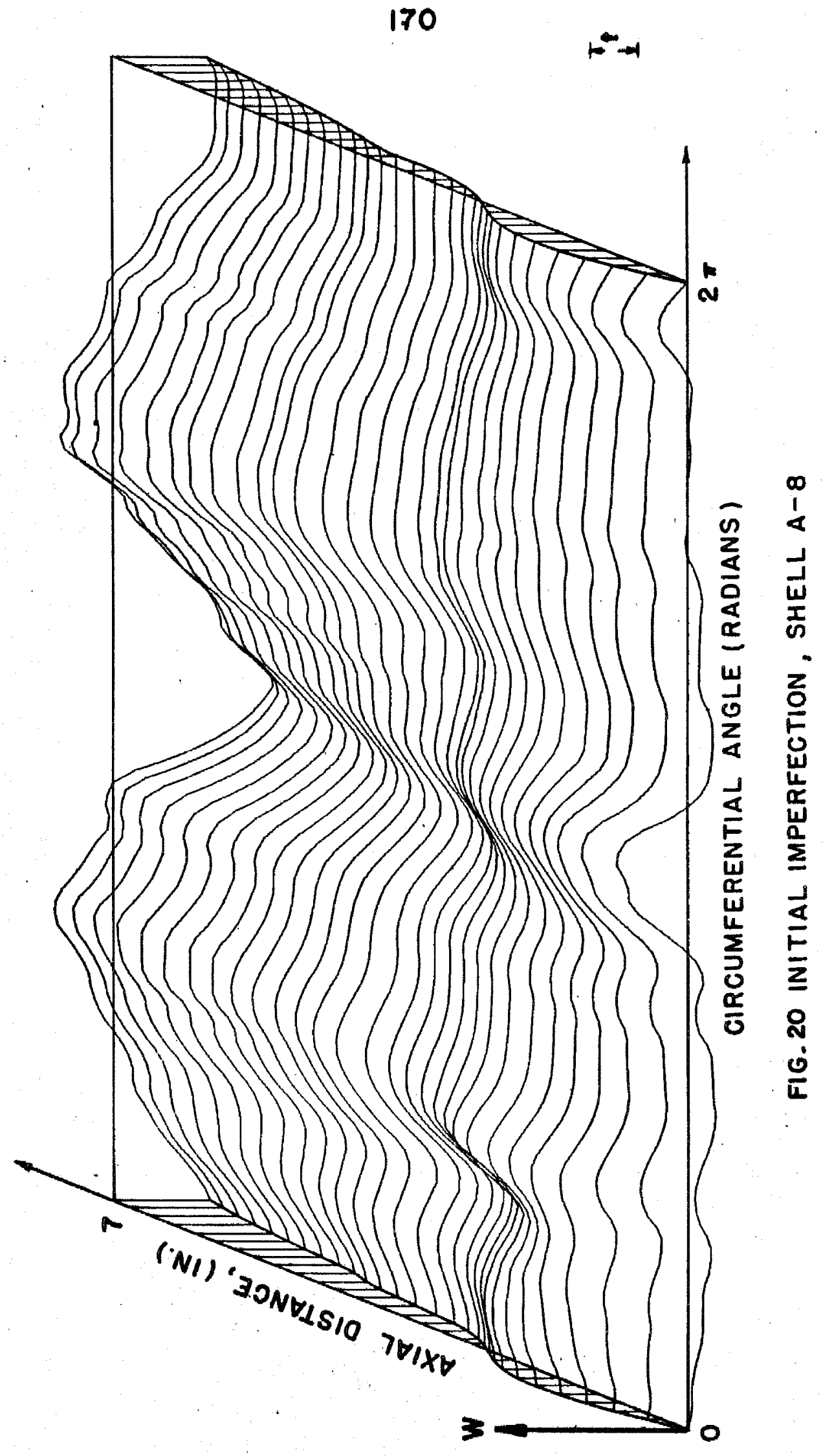




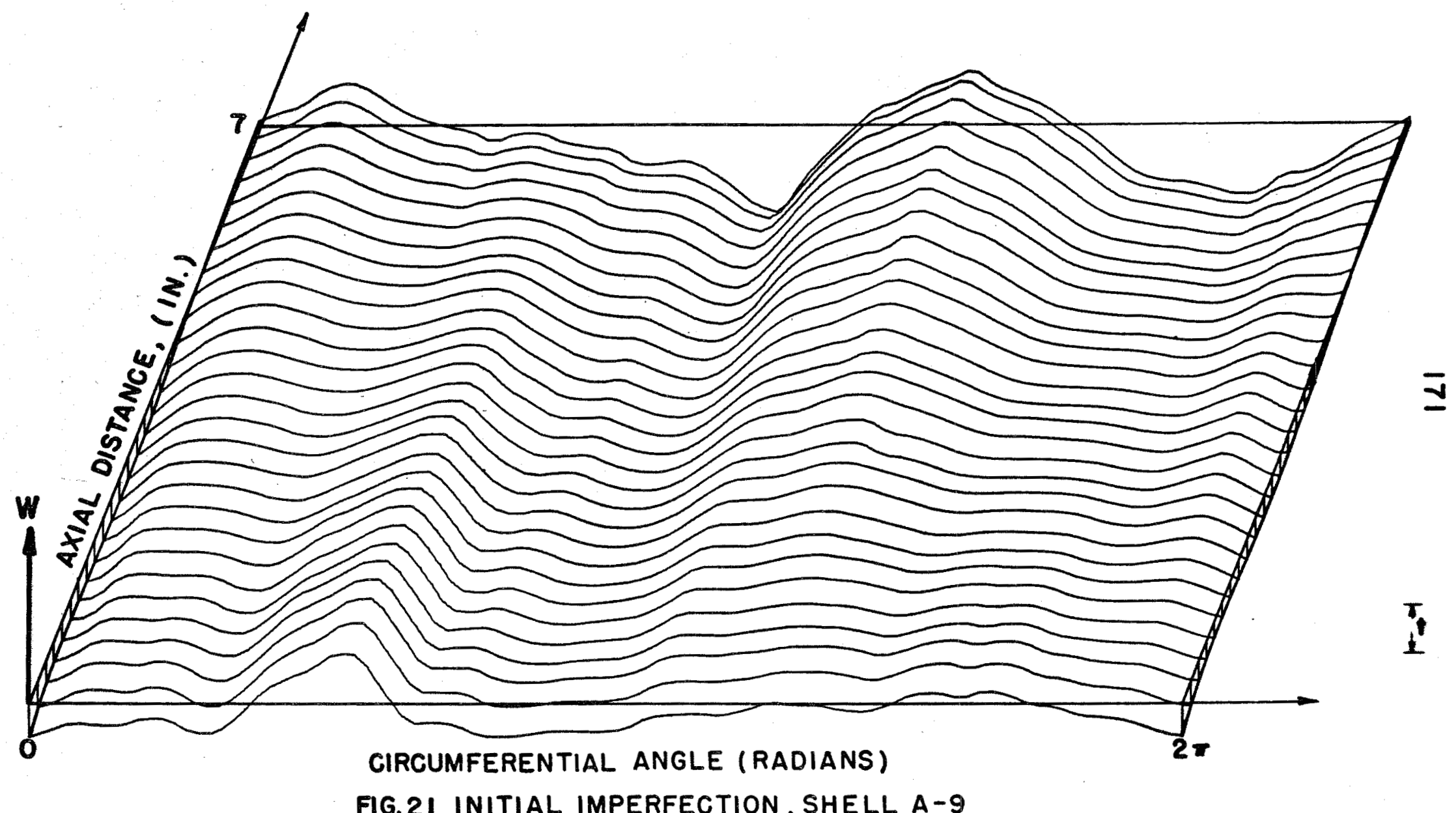

FIG. 2 I INITIAL IMPERFECTION, SHELL A-9 


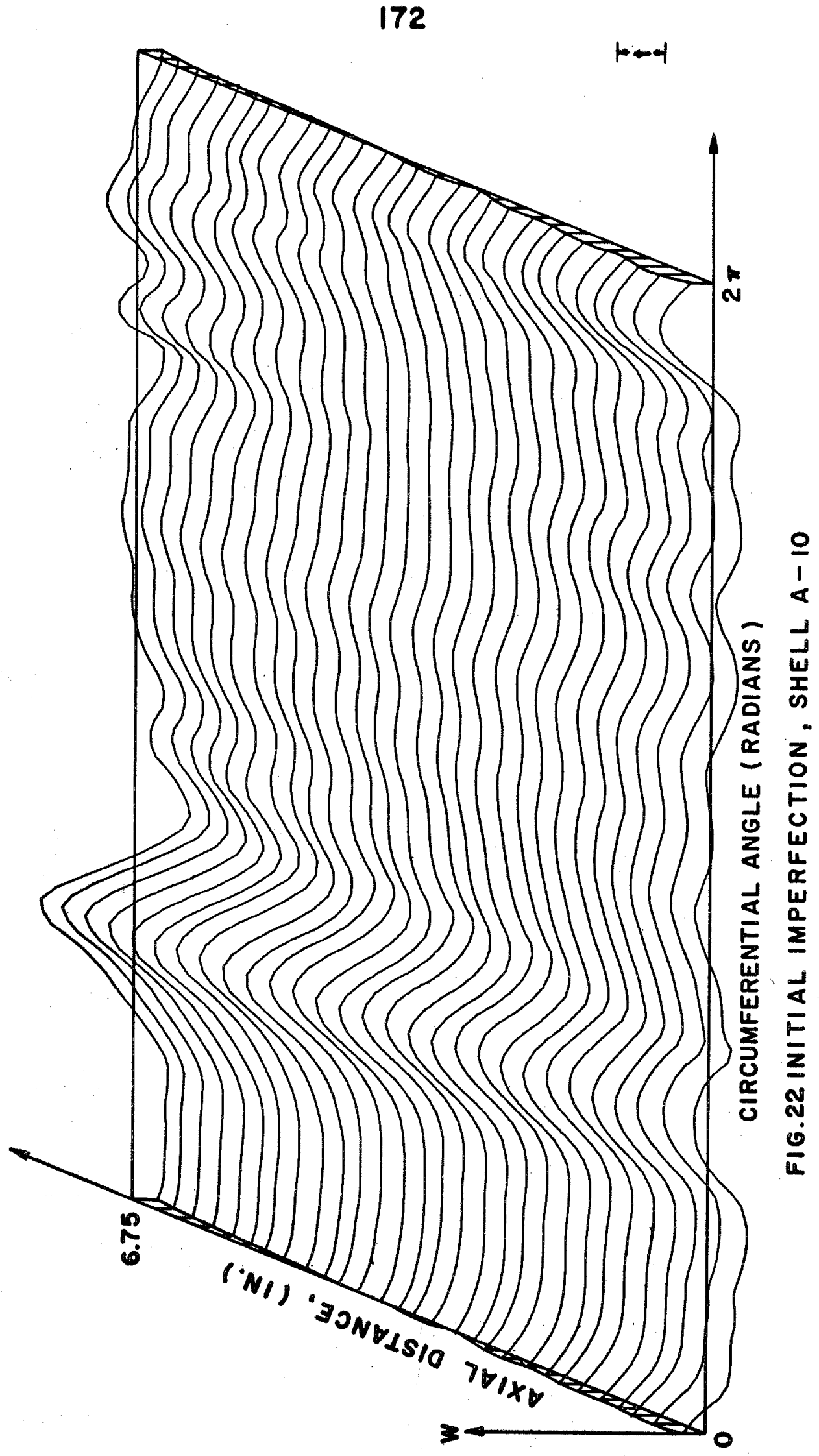




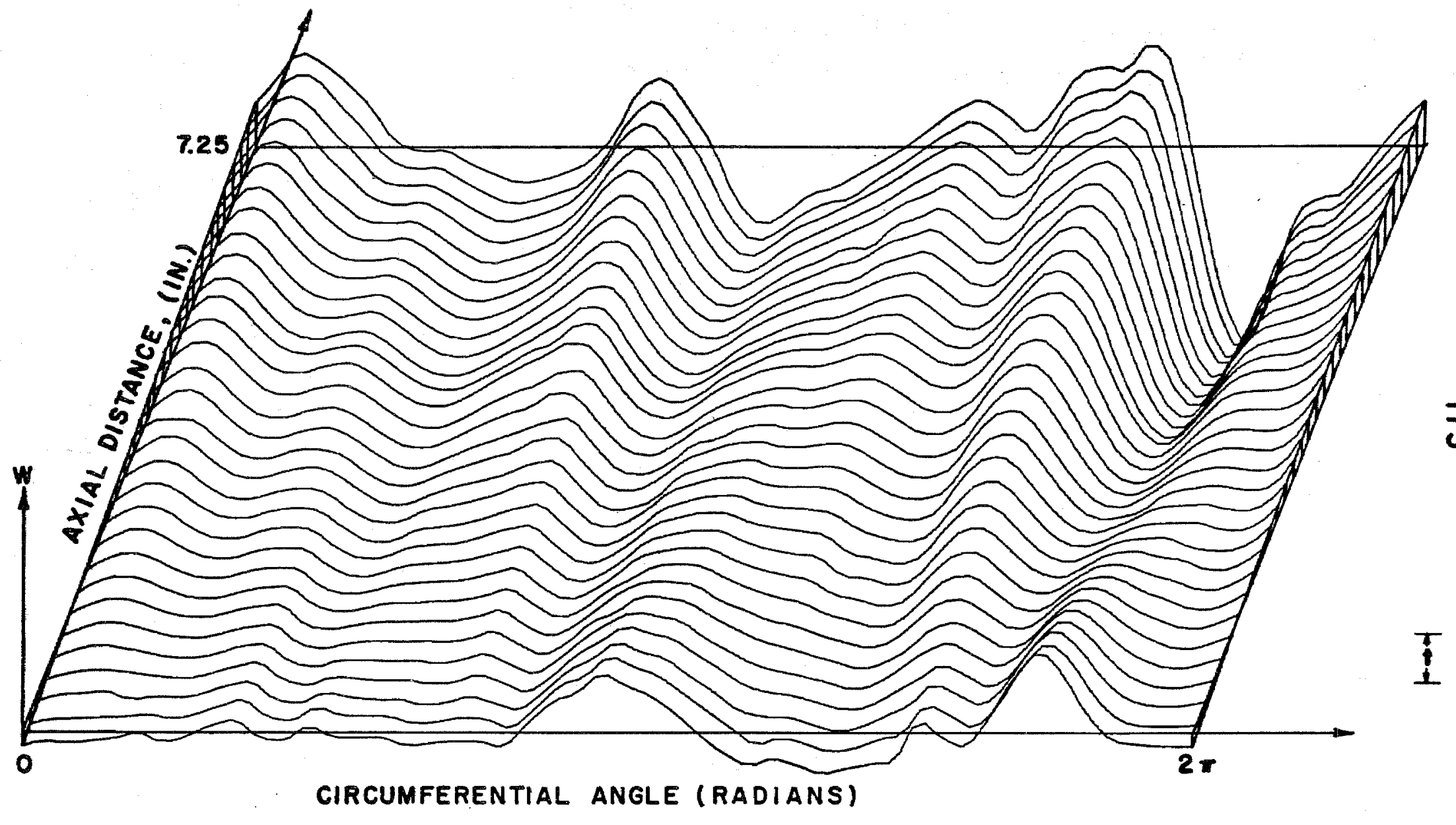

$\bar{v}$

FIG.23 INITIAL IMPERFECTION, SHELL A- 12 


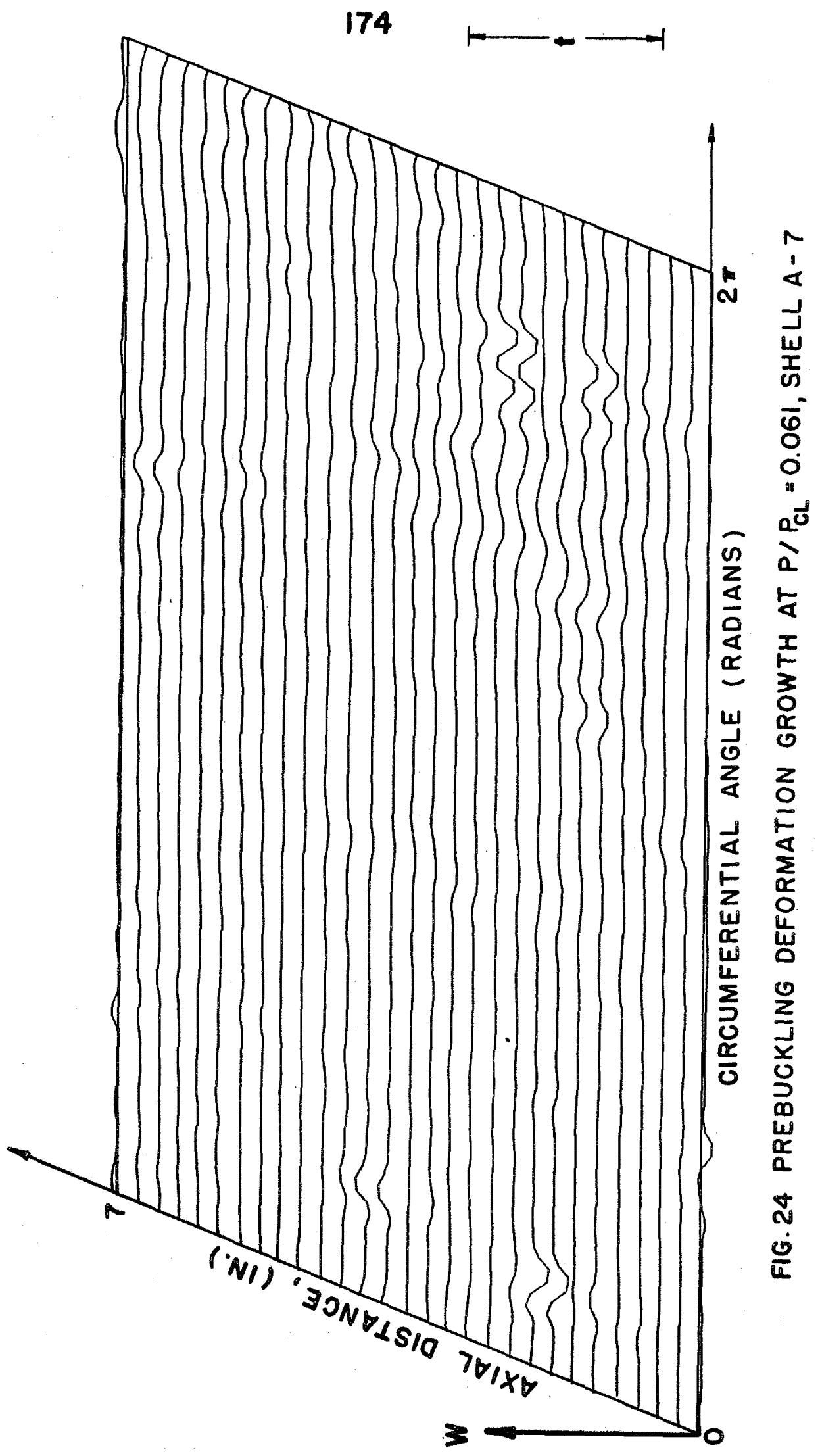




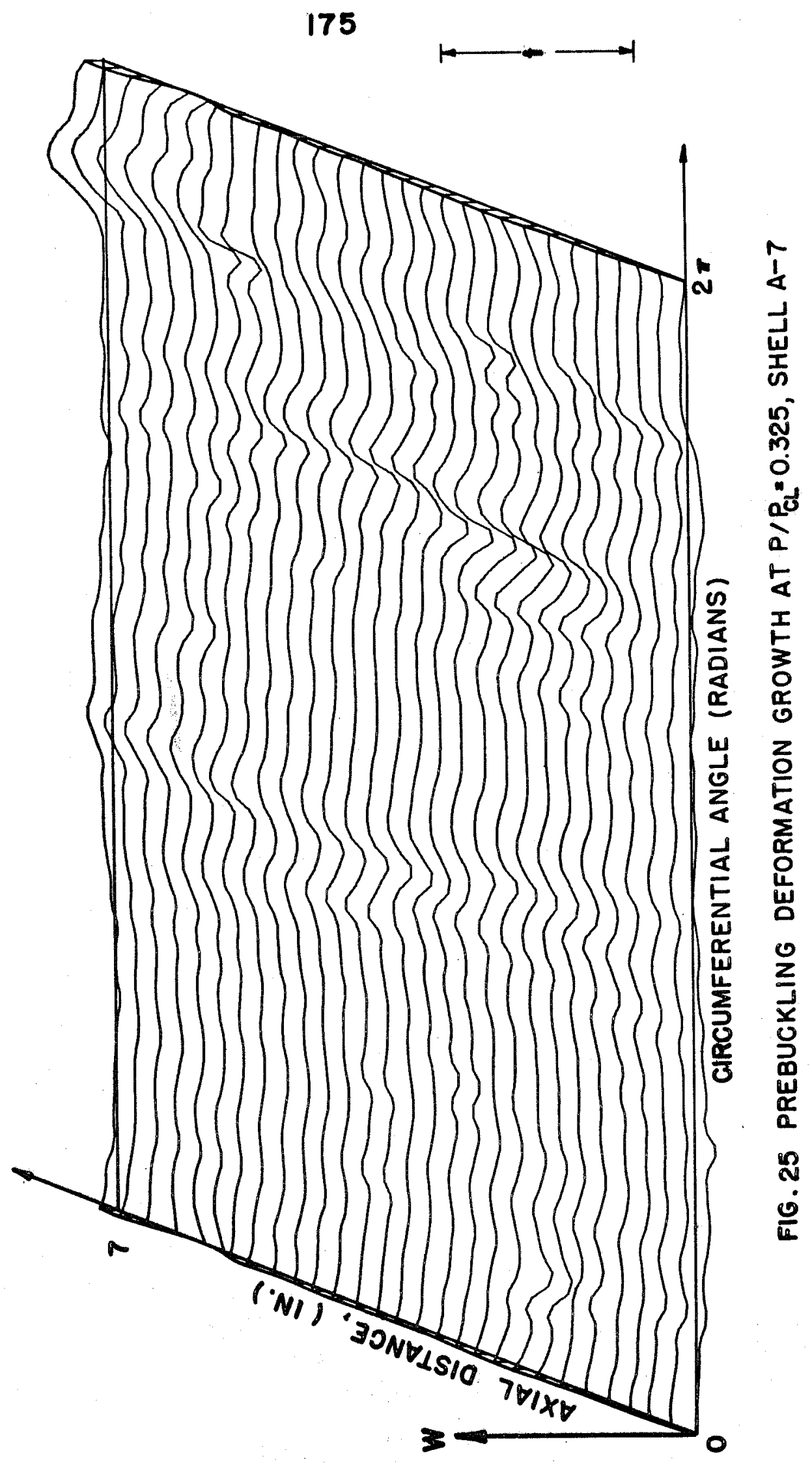




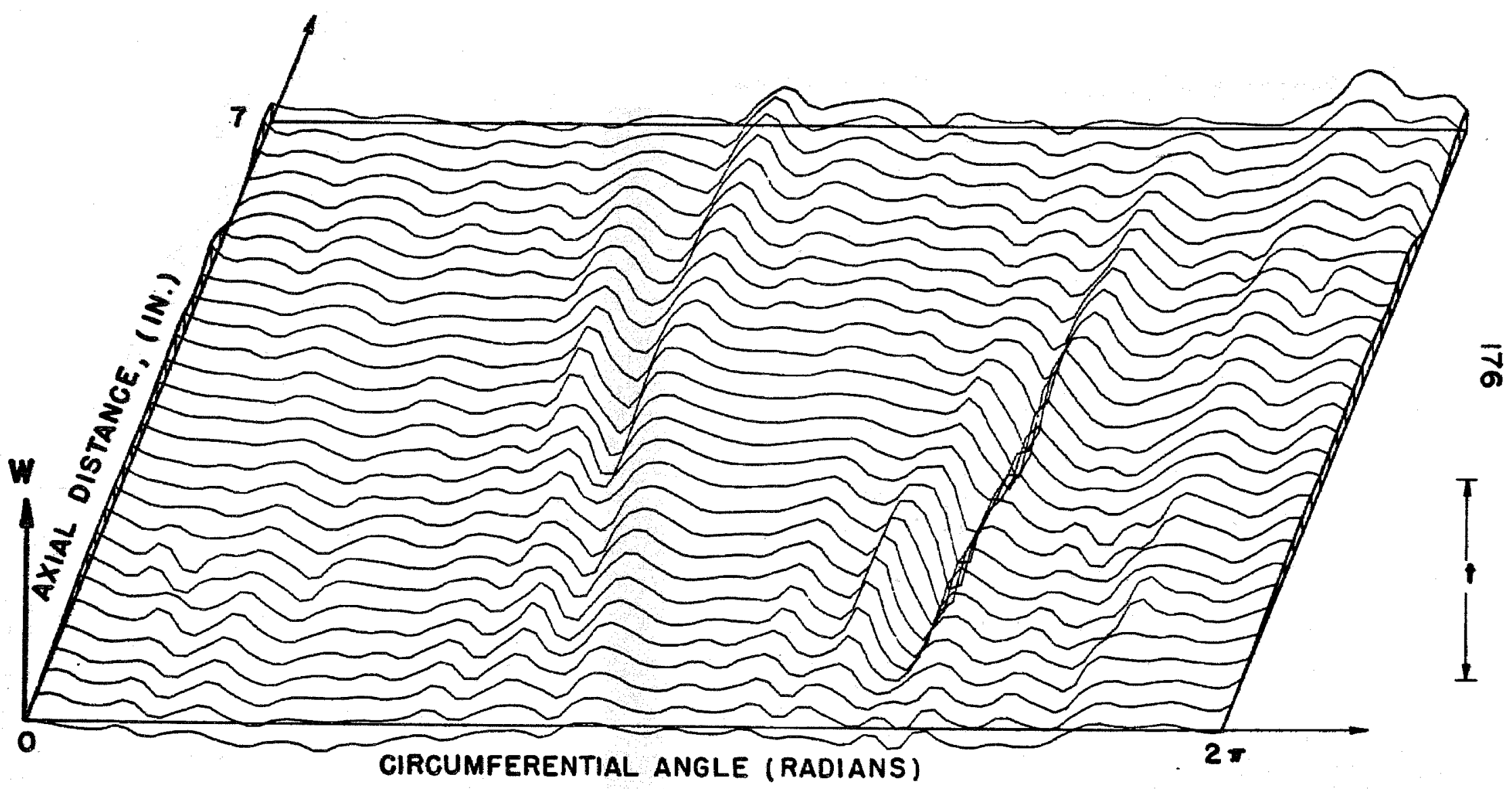

FIG.26 PREBUGKLING DEFORMATION GROWTH AT P/P $P_{C L}=0.539$, SHELL A-7 


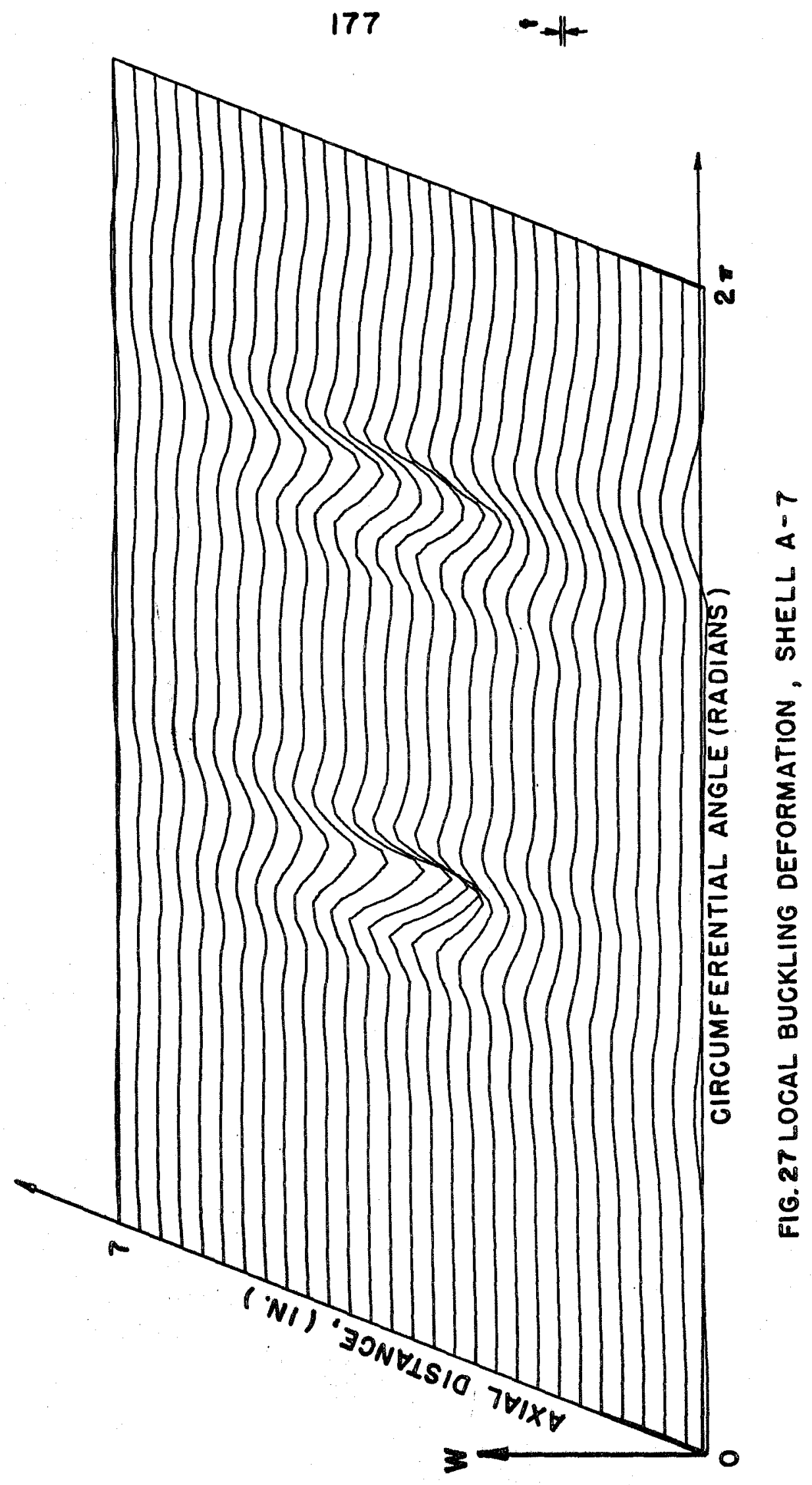




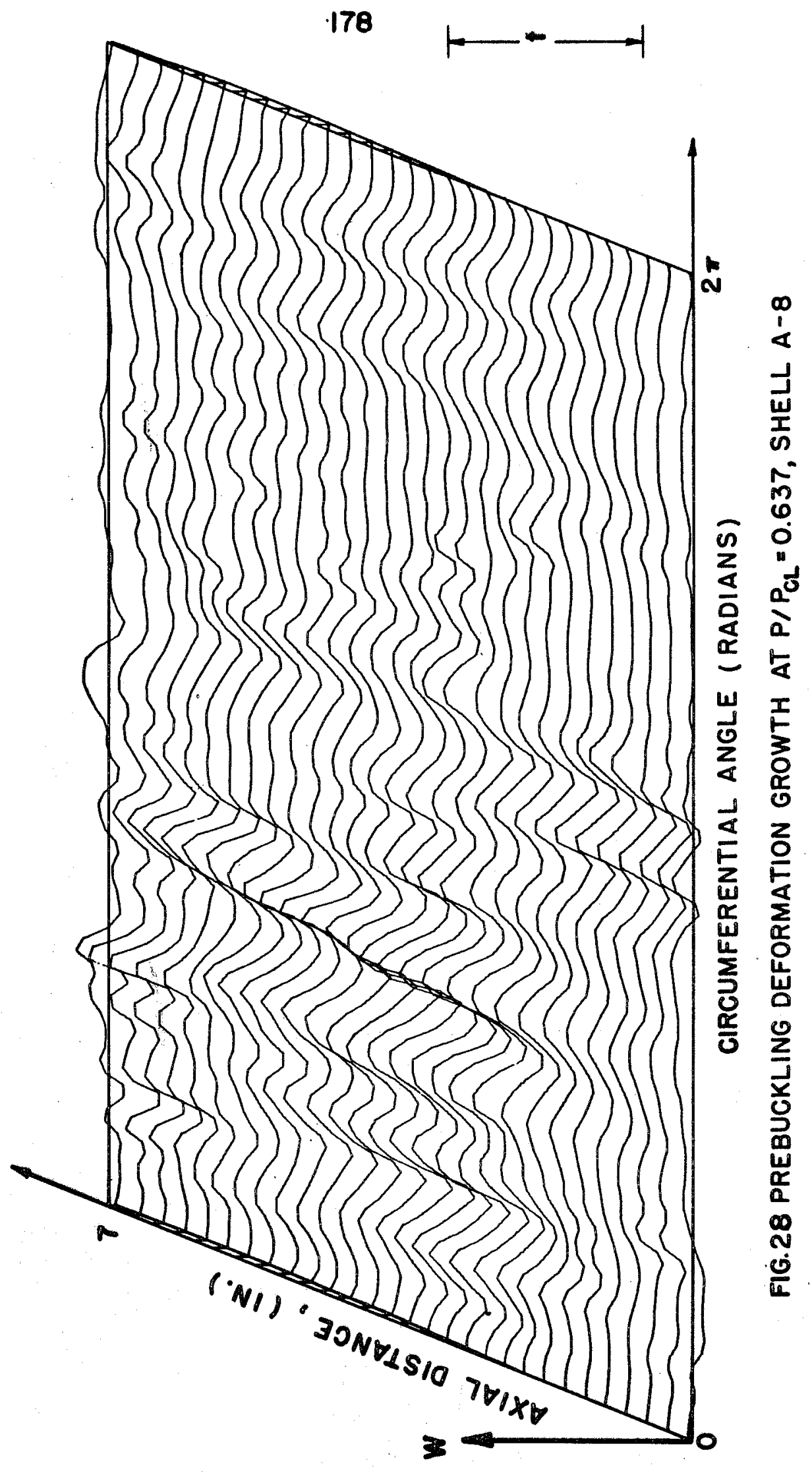




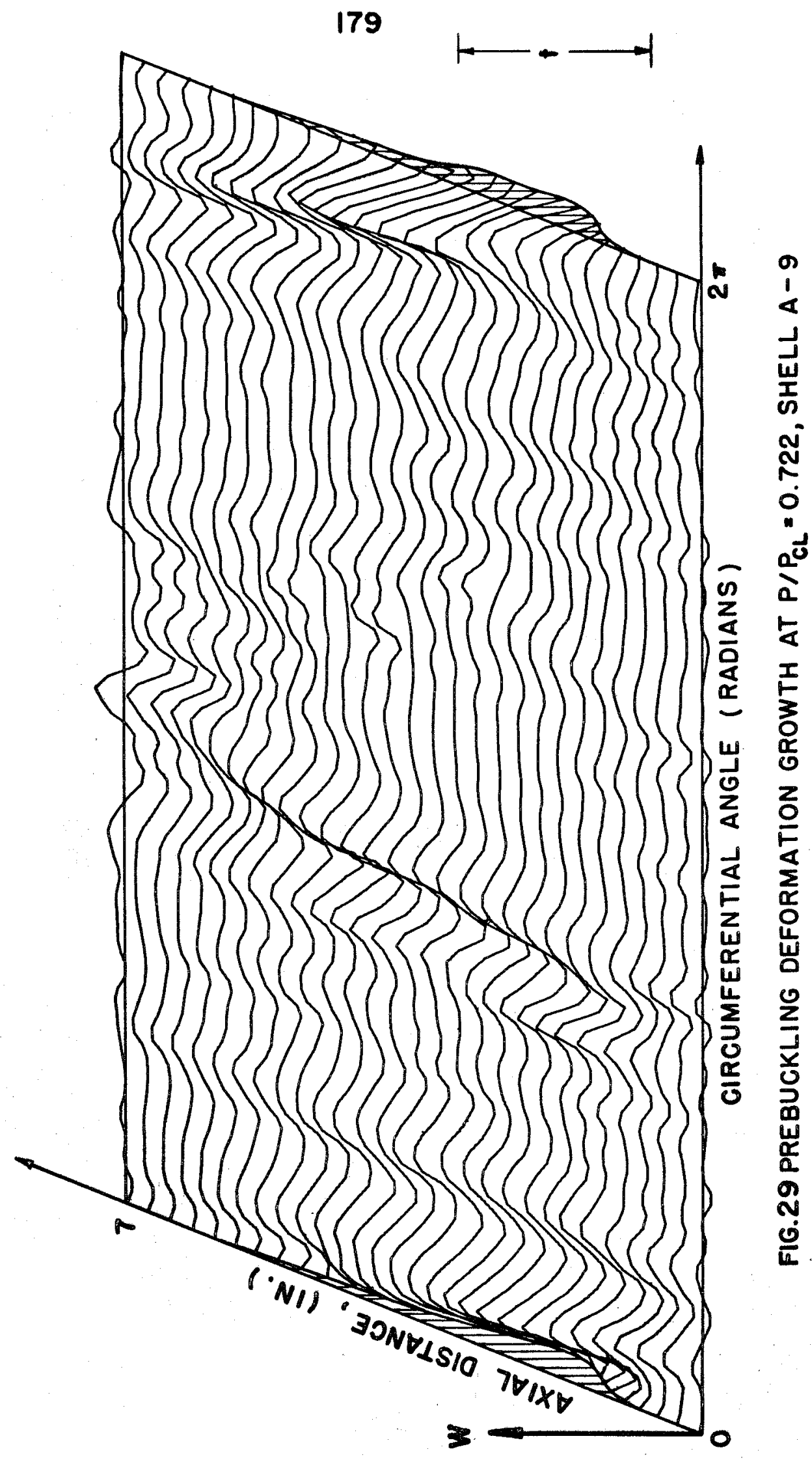




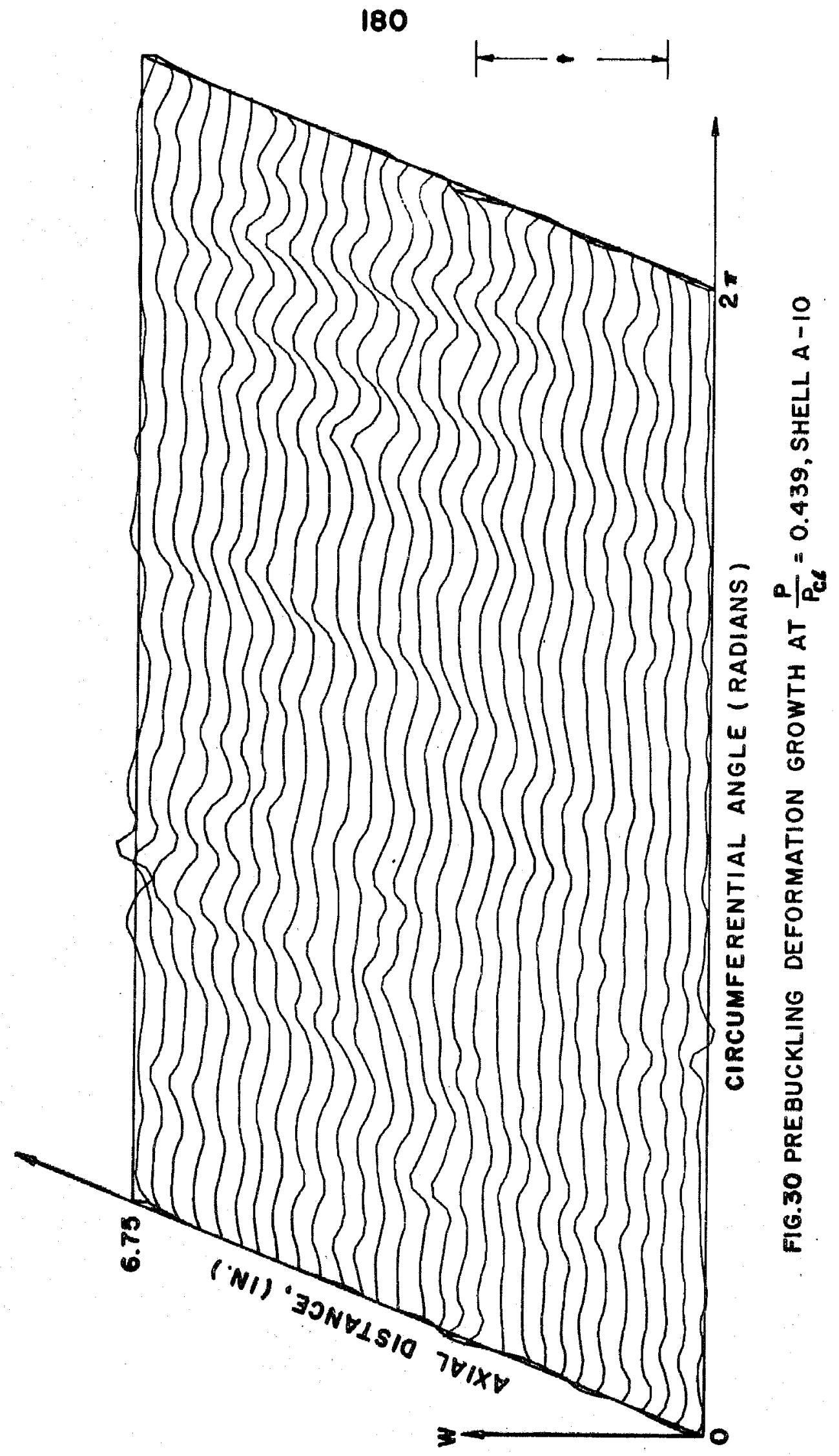




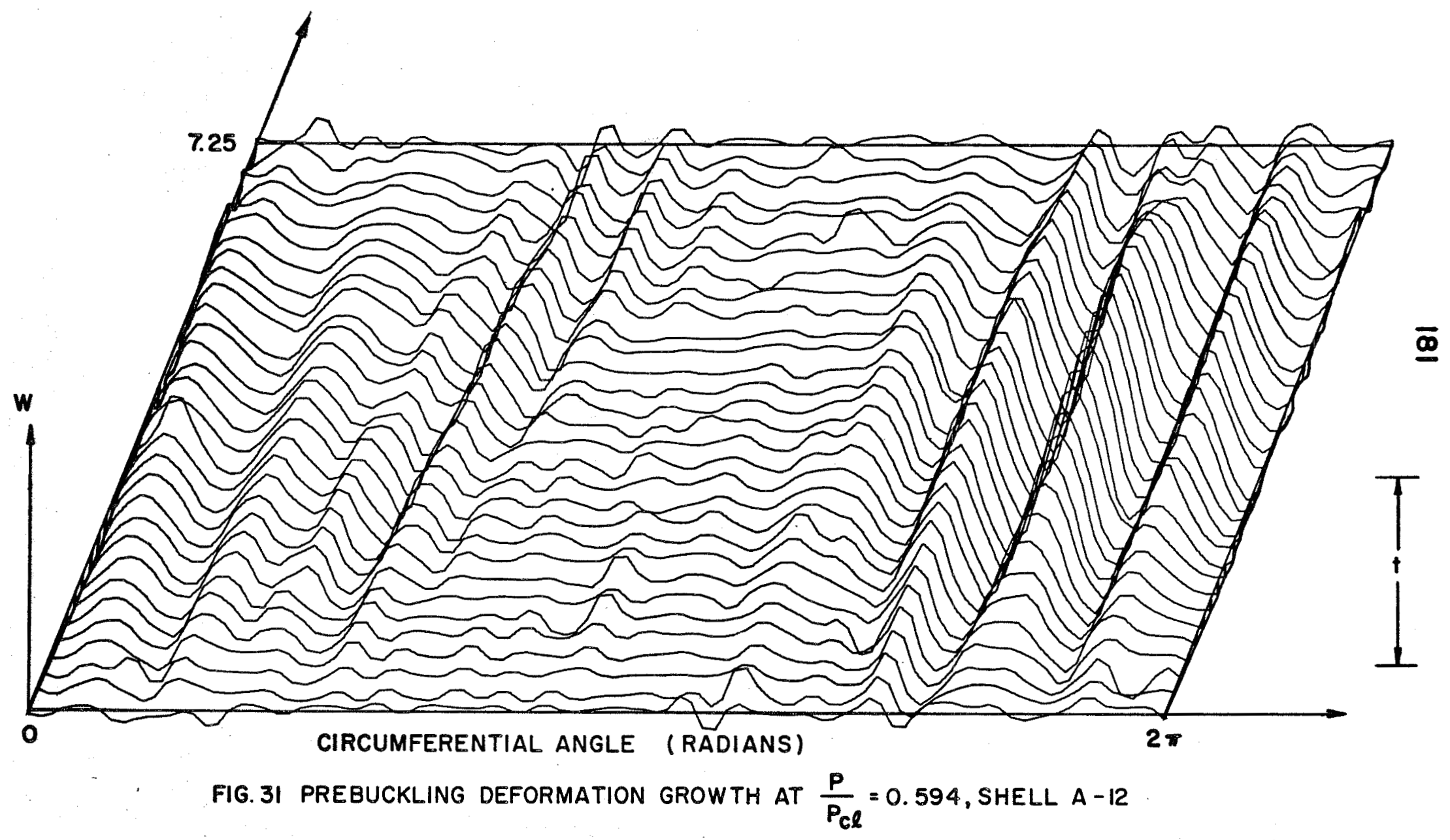




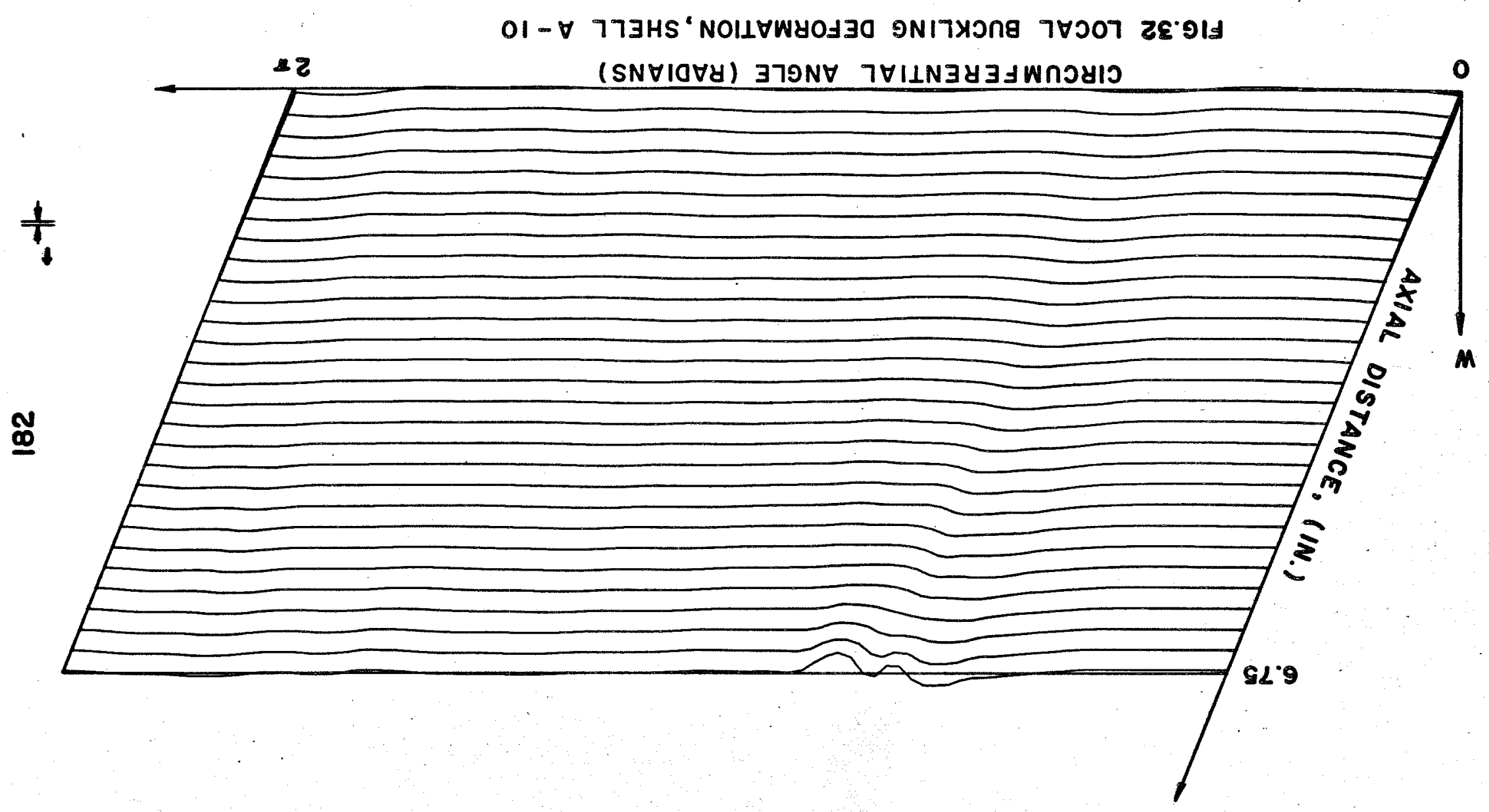




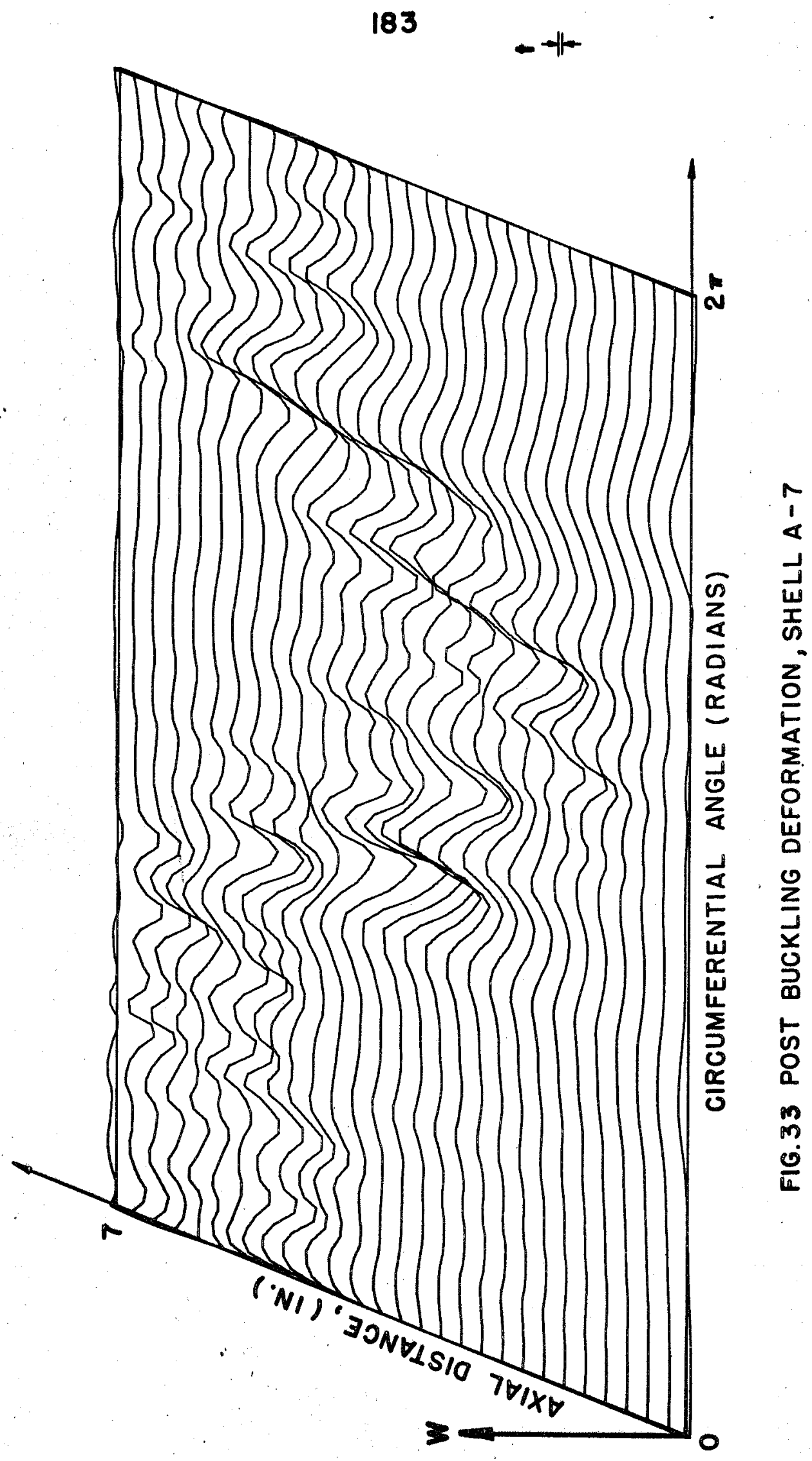




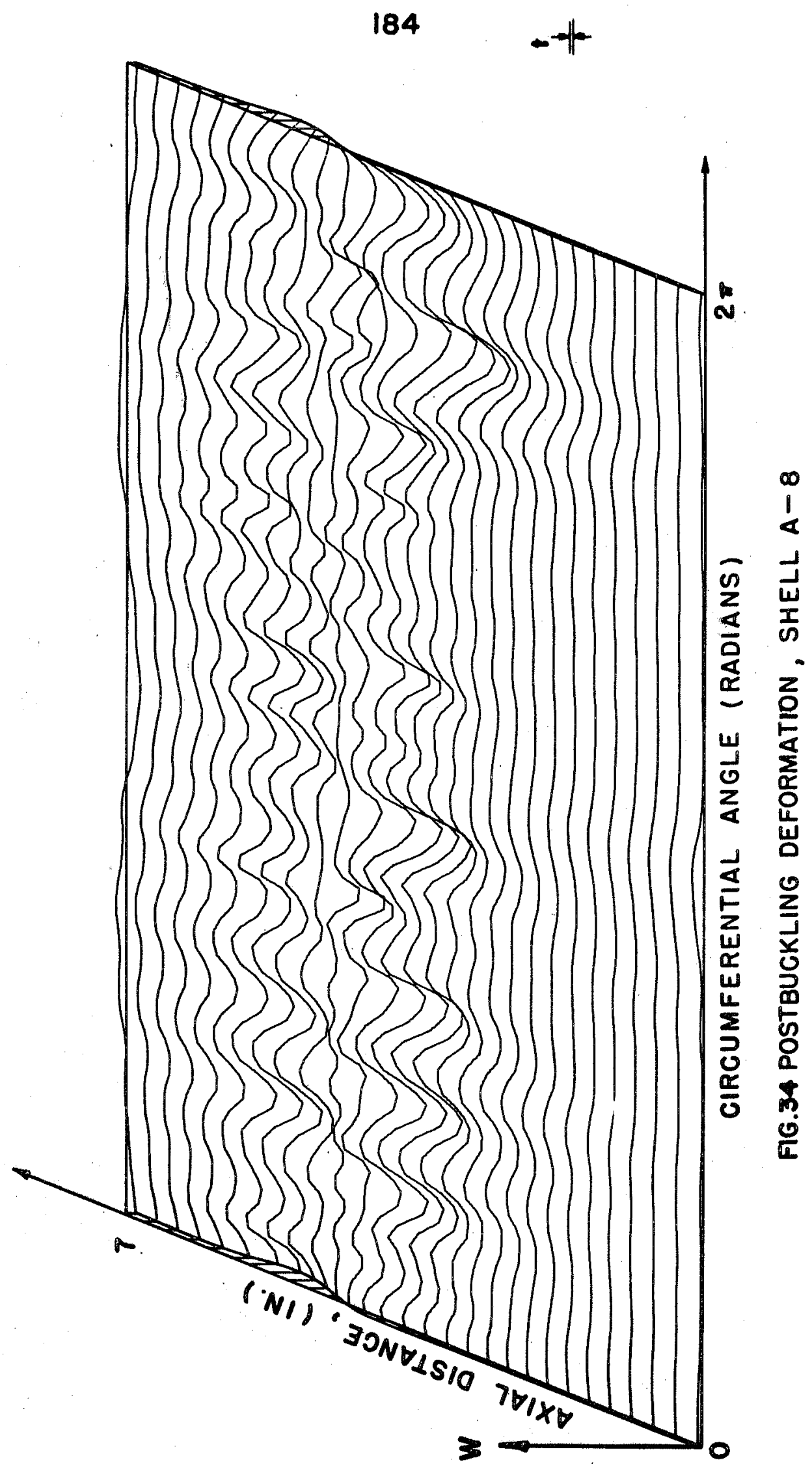




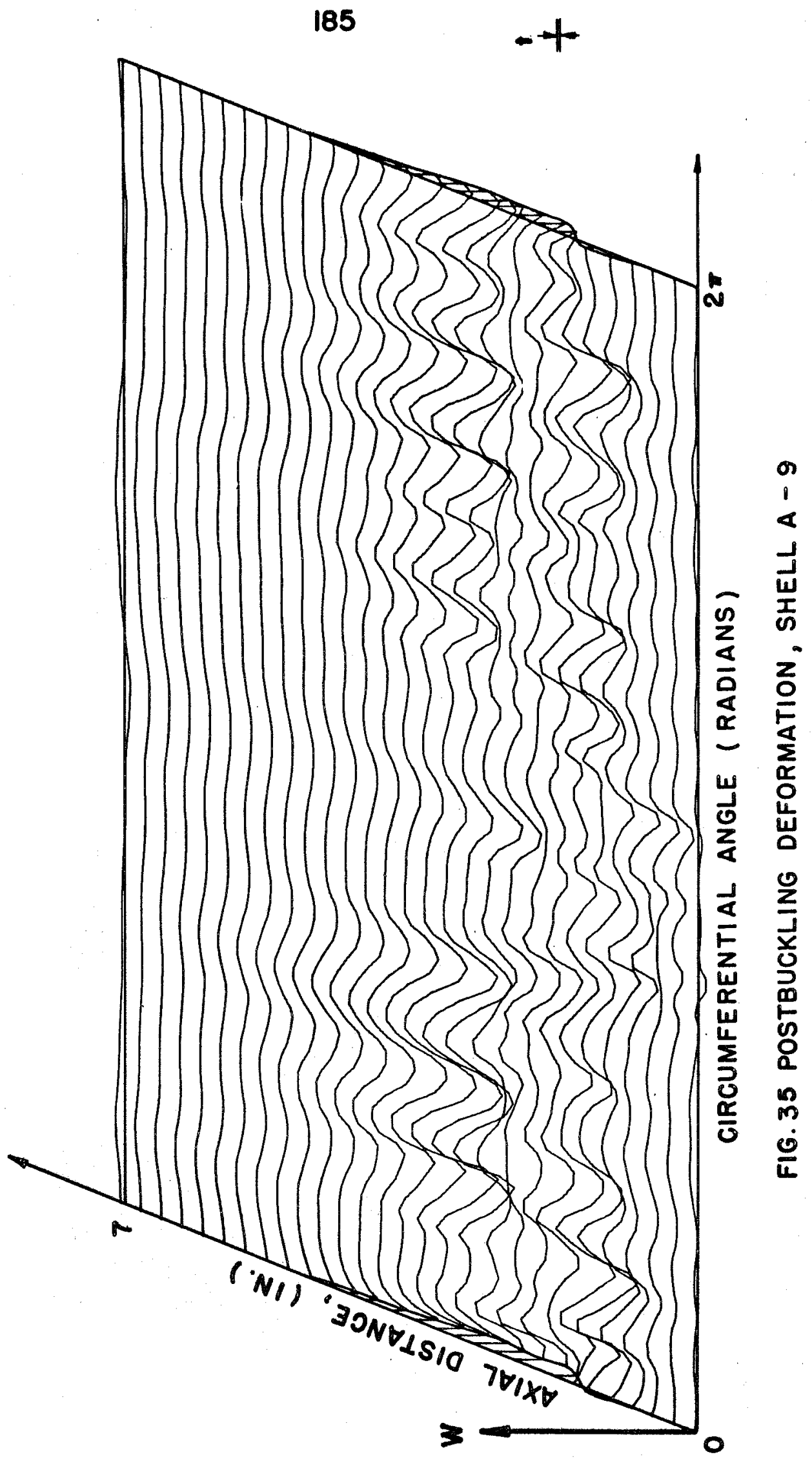




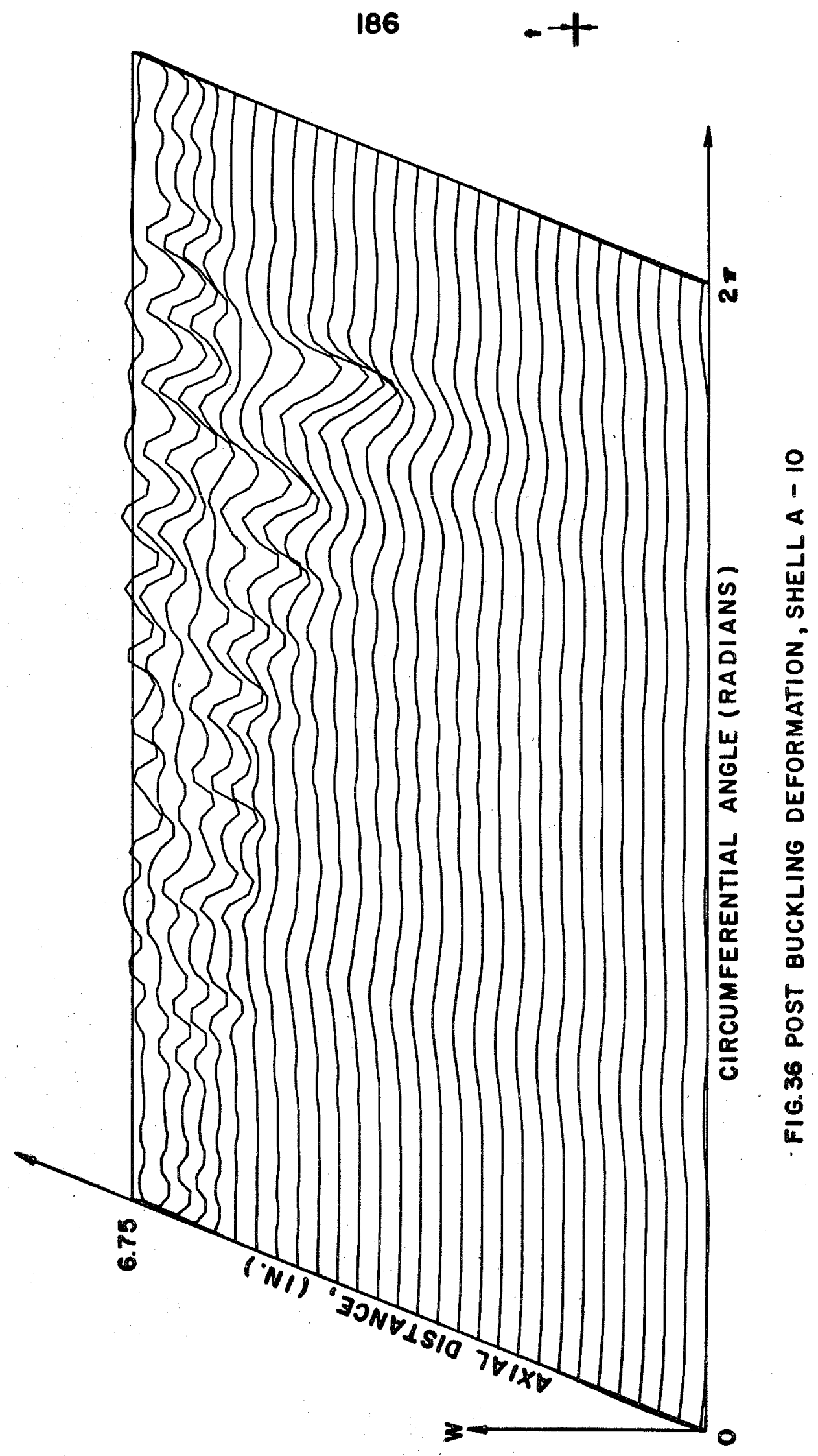




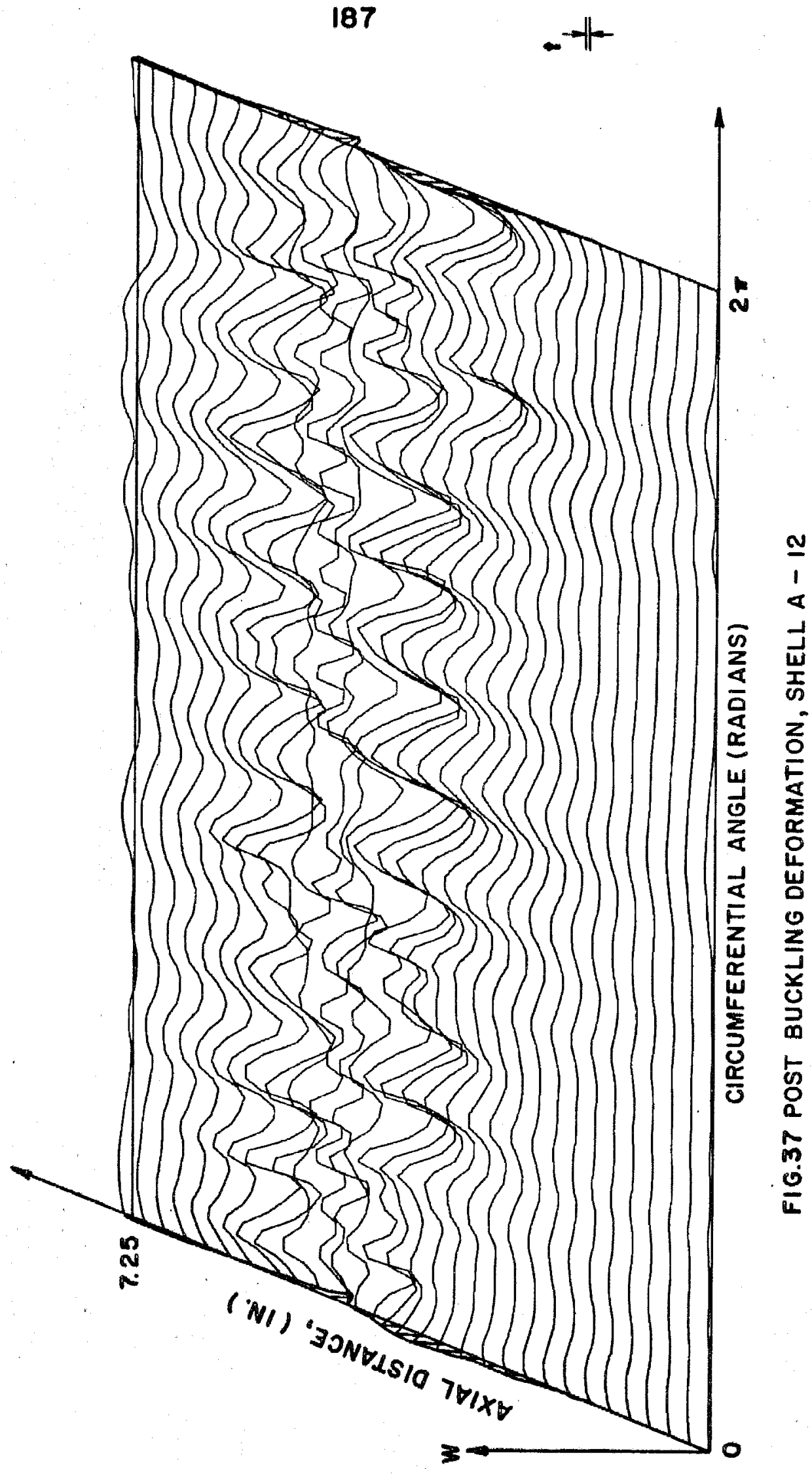


188
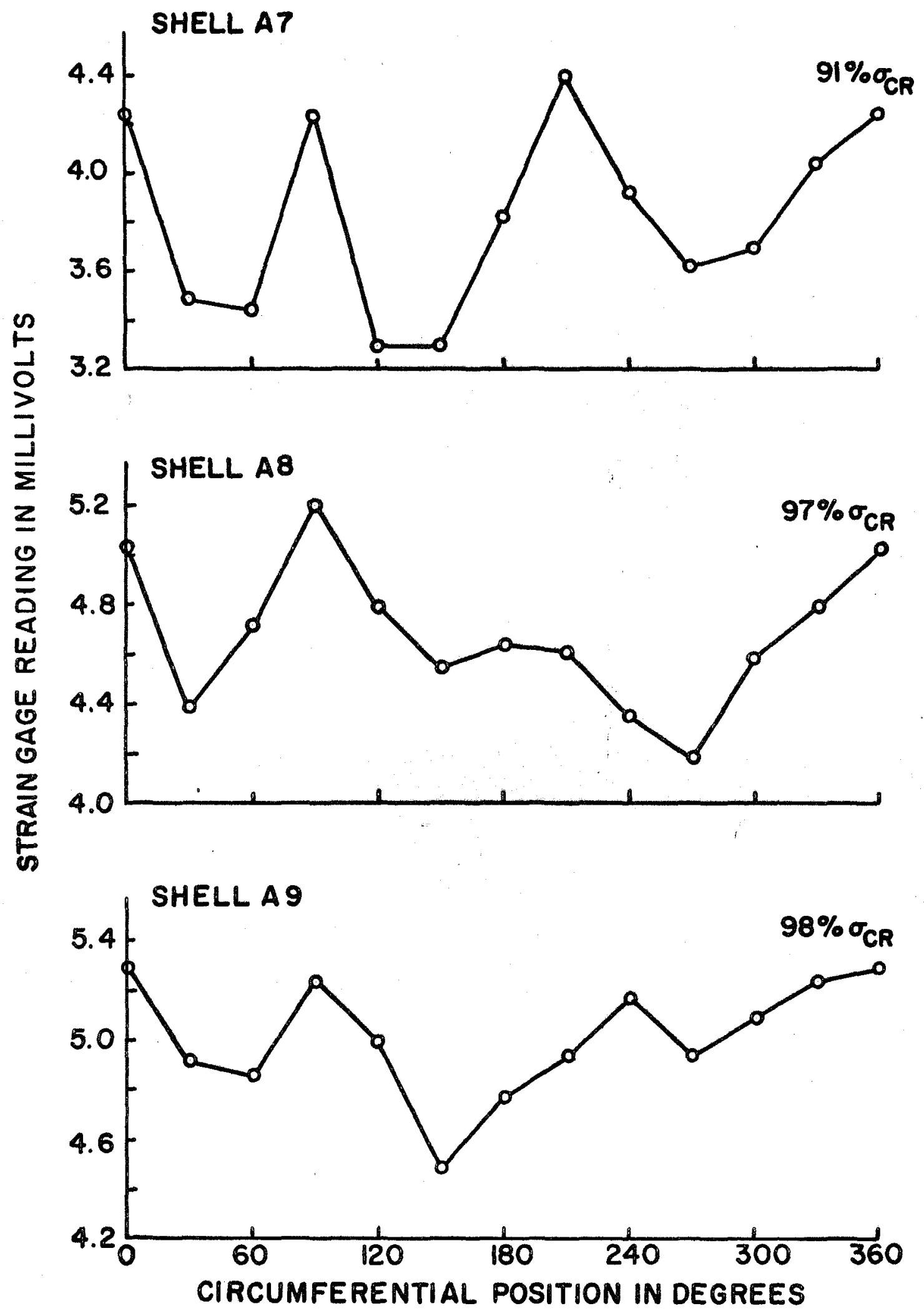

FIG.38 LOAD DISTRIBUTION NEAR BUCKLING 


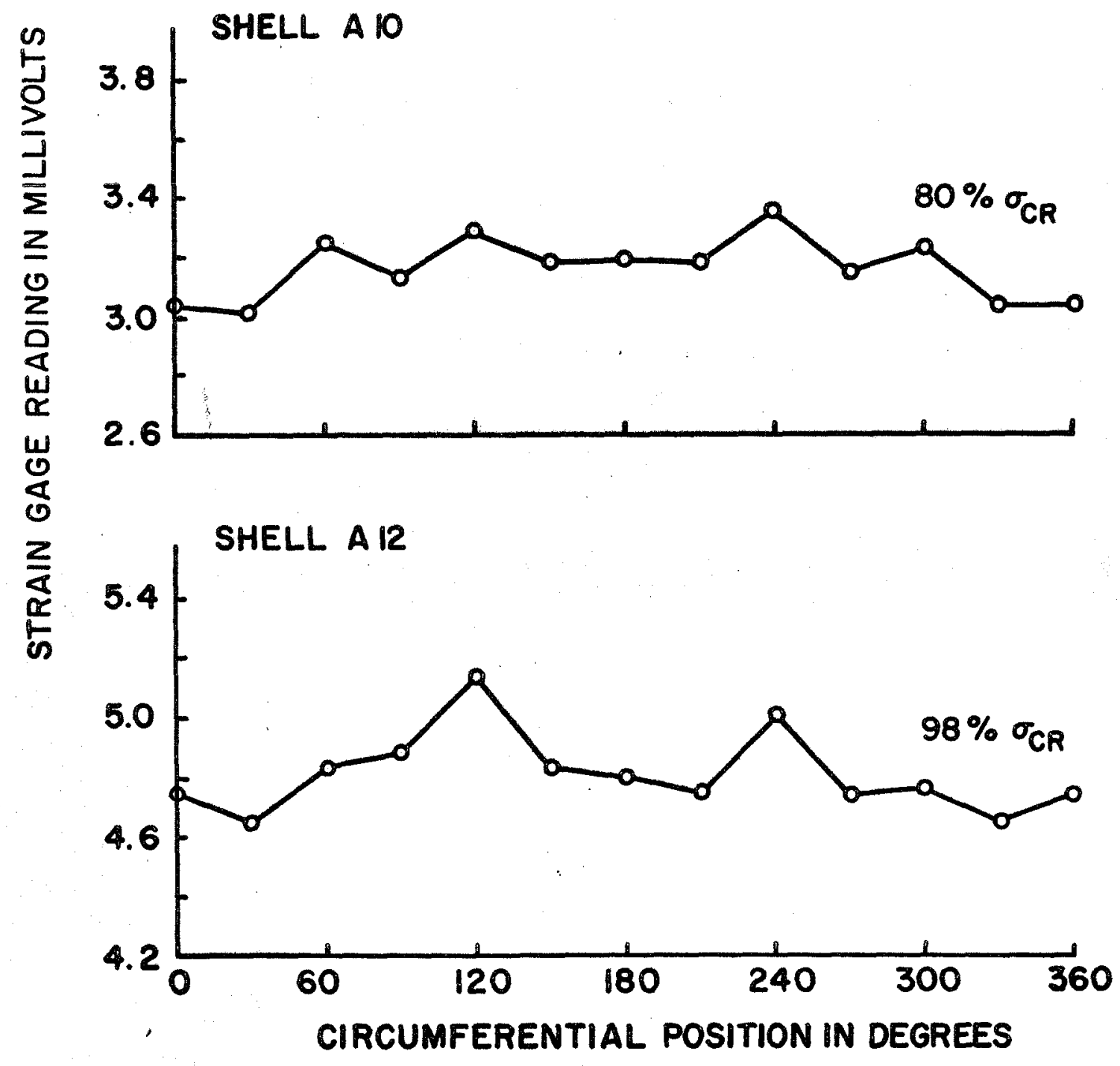

FIG.39 LOAD DISTRIBUTION NEAR BUCKLING 


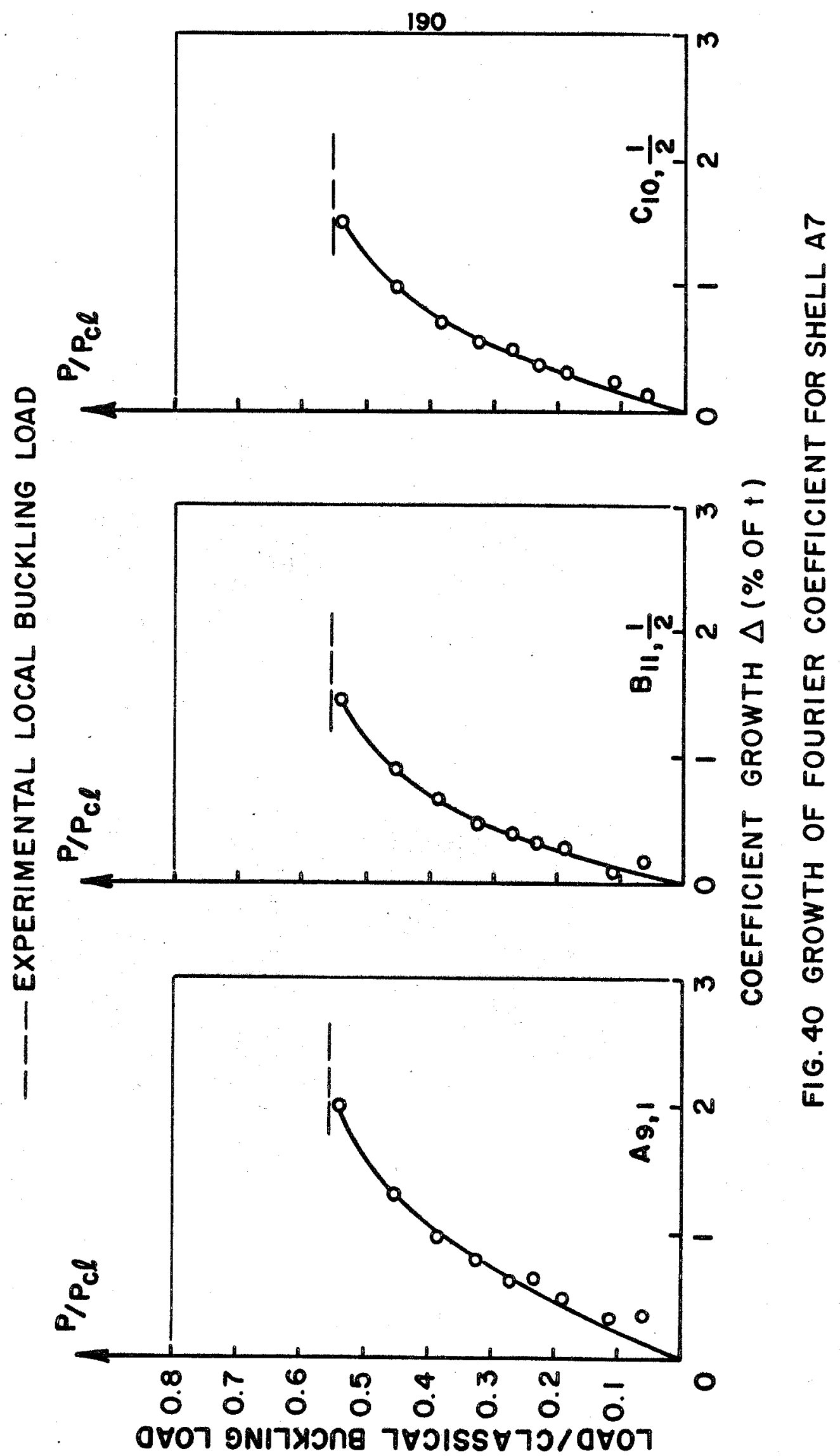


- - EXPERIMENTAL BUCKLING LOAD

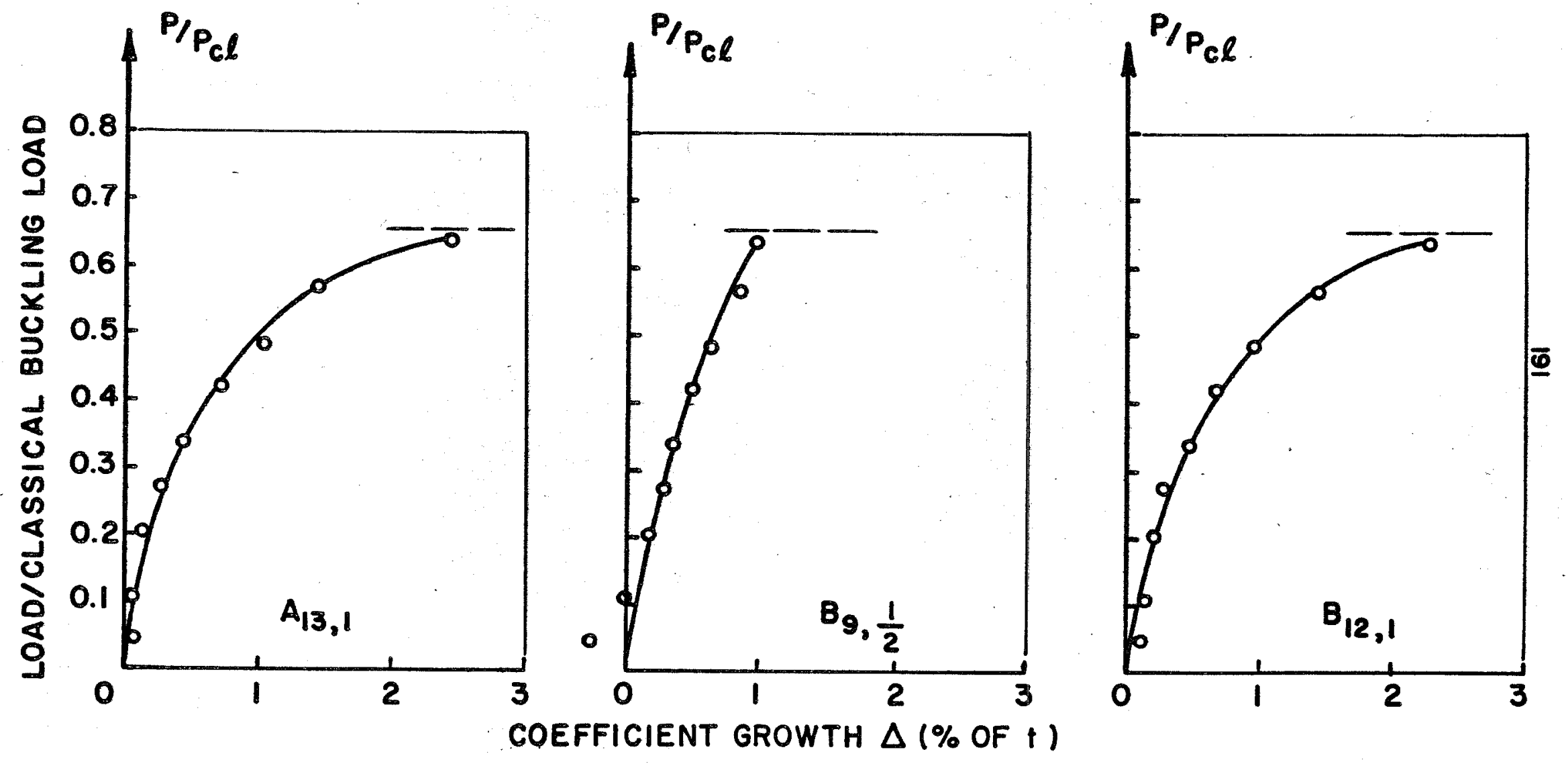

FIG. 41 GROWTH OF FOURIER COEFFICIENTS FOR SHELL A8 


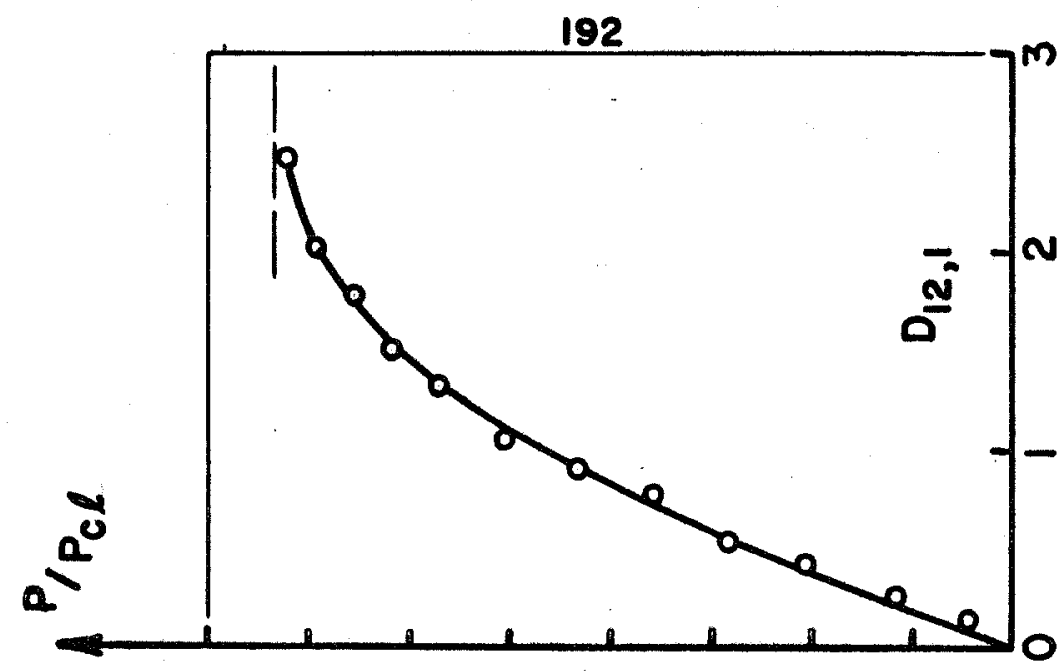

$\frac{9}{4}$
$\frac{1}{4}$
$\frac{1}{9}$

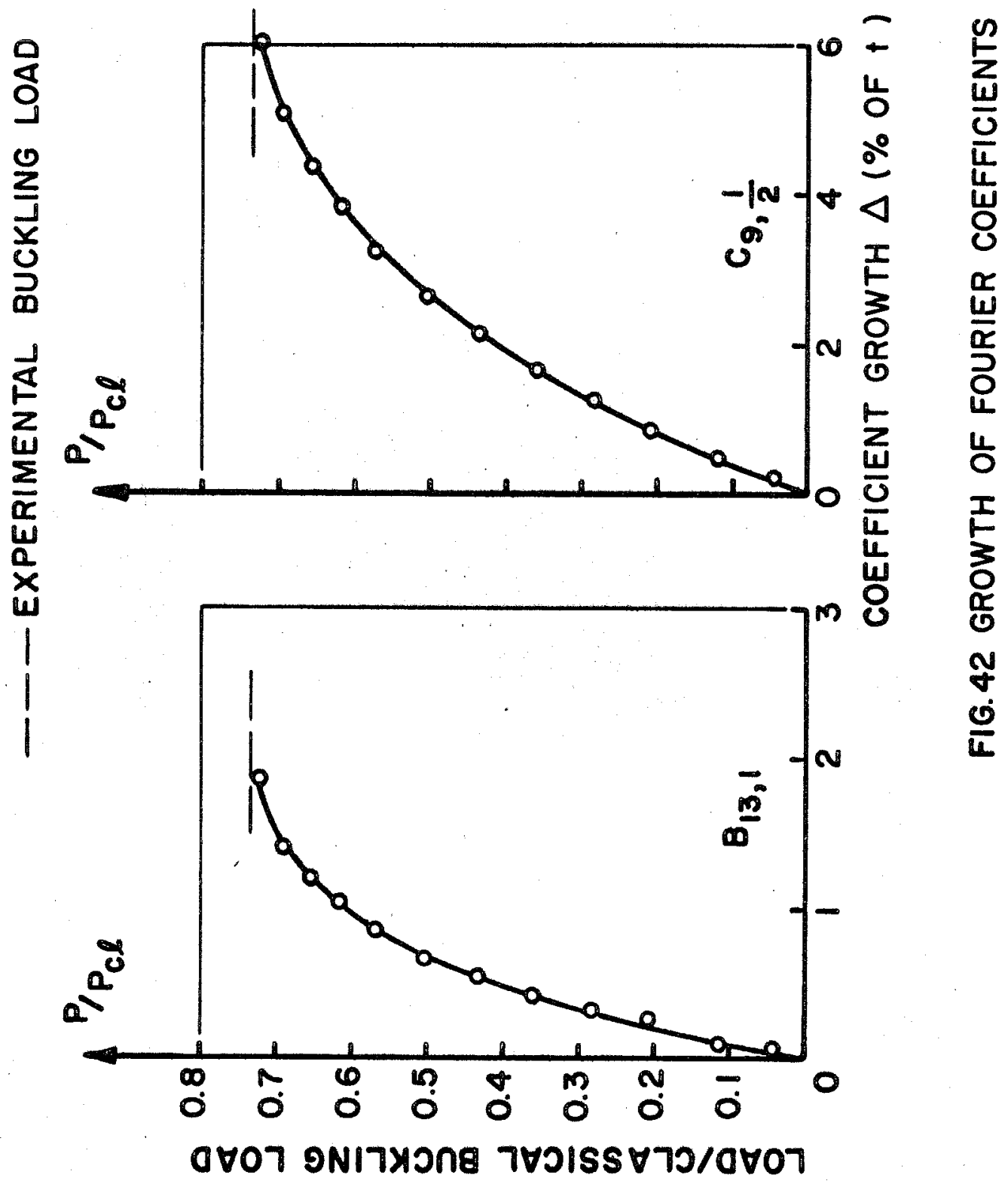




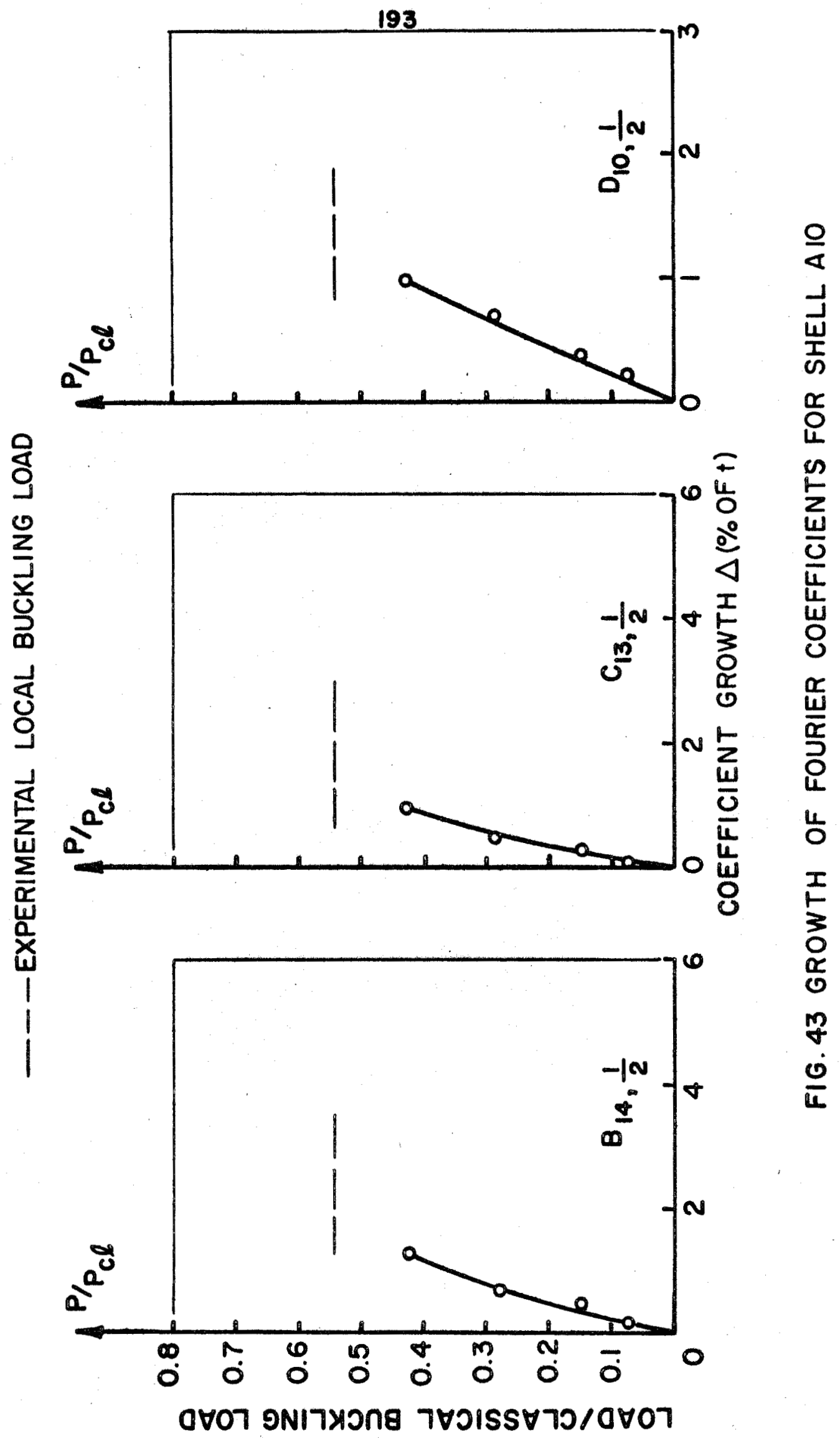



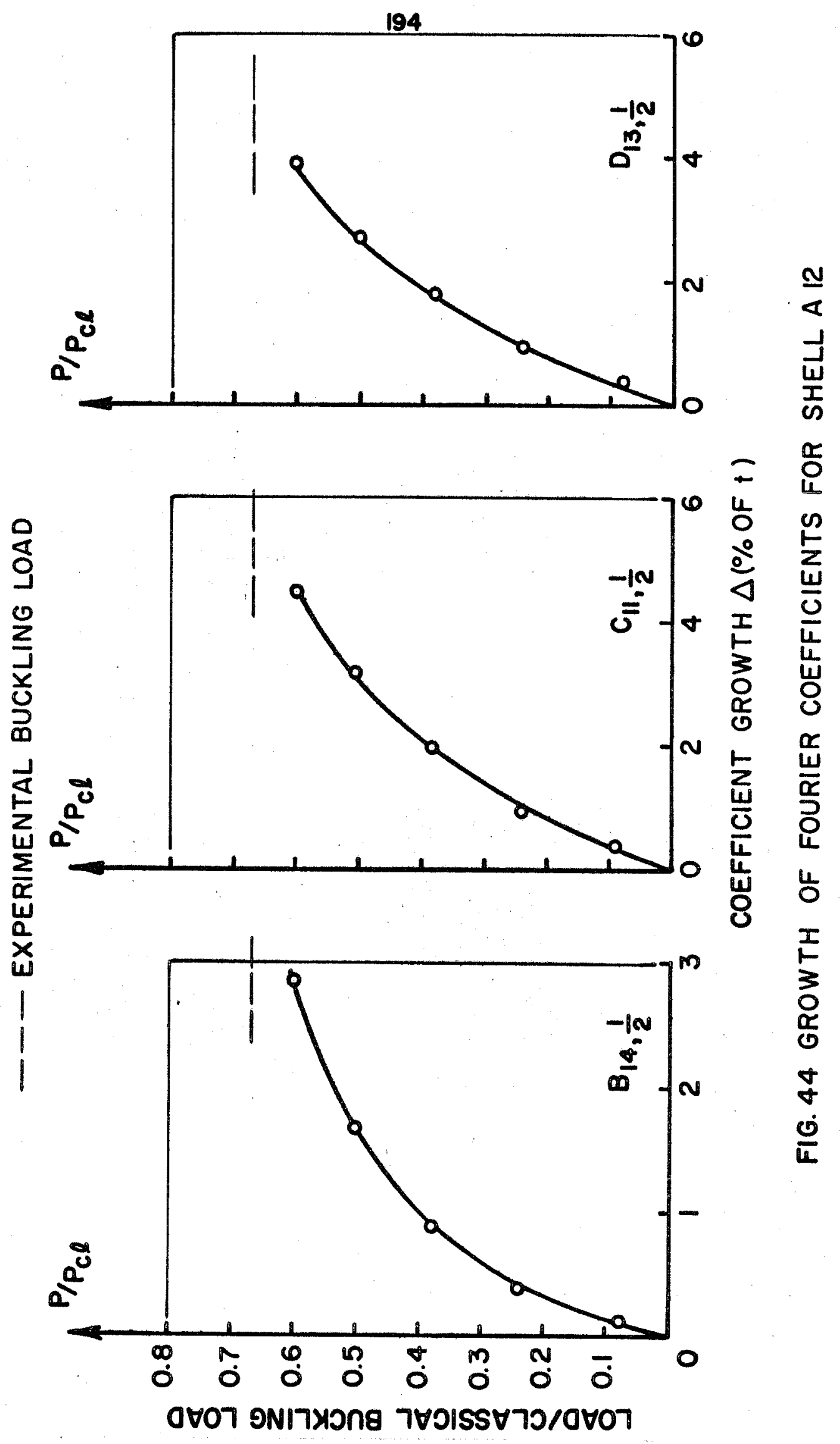
195

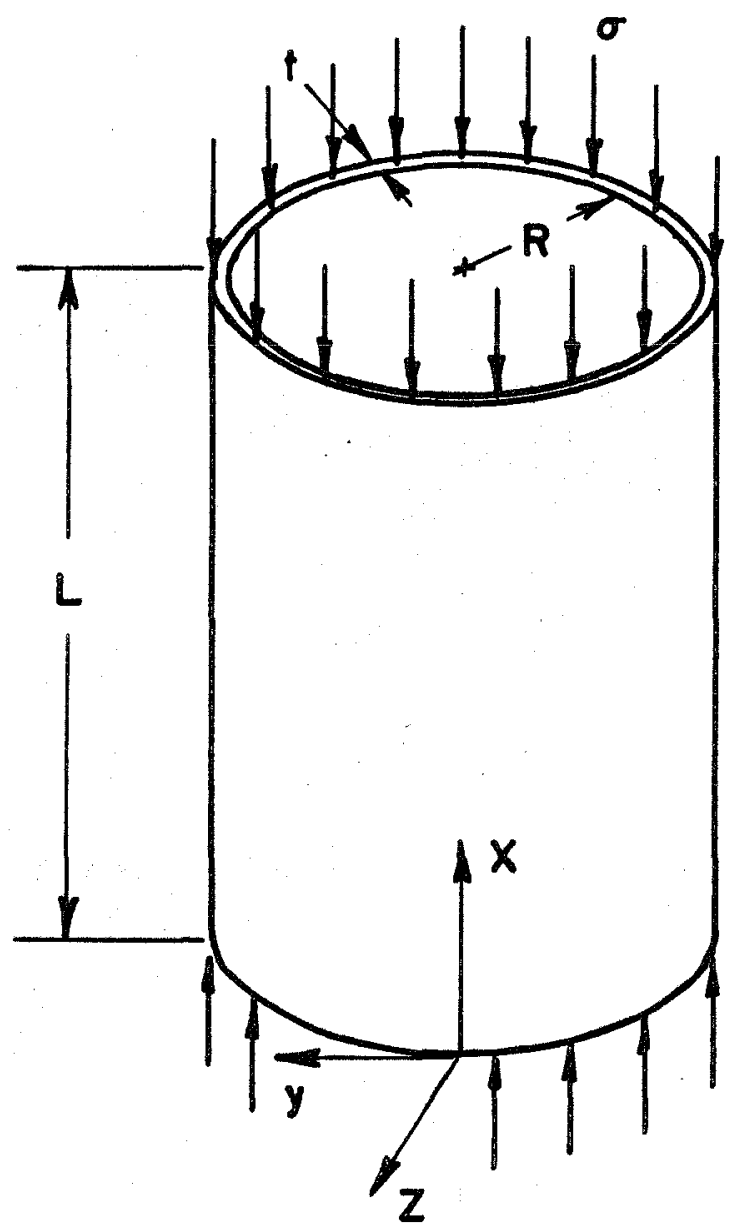

FIG. 45 SHELL GEOMETRY AND COORDINATE SYSTEM 

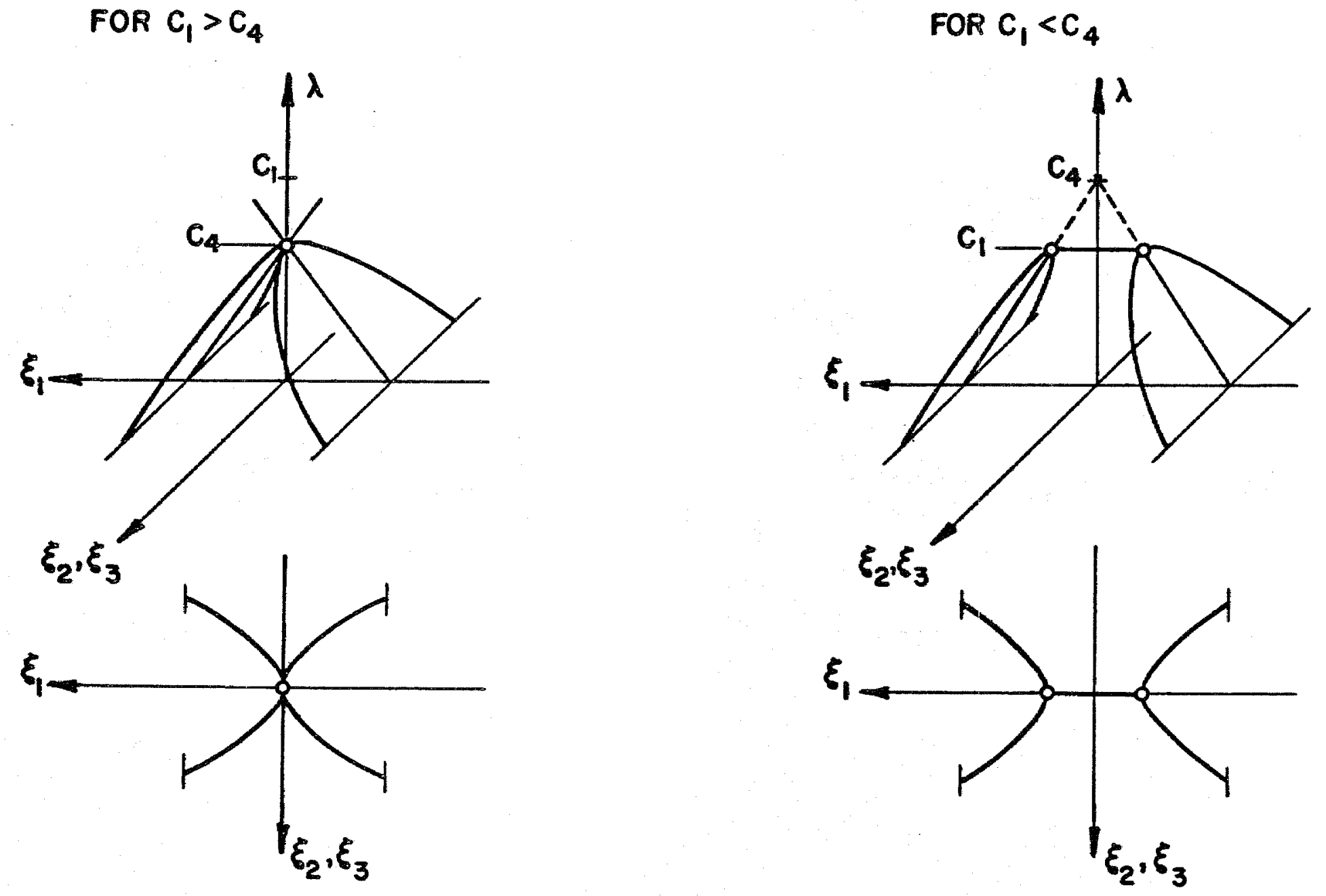

FIG. 46 POST-BUCKLING EQUILIBRIUM PATHS FOR PERFECT SHELLS 

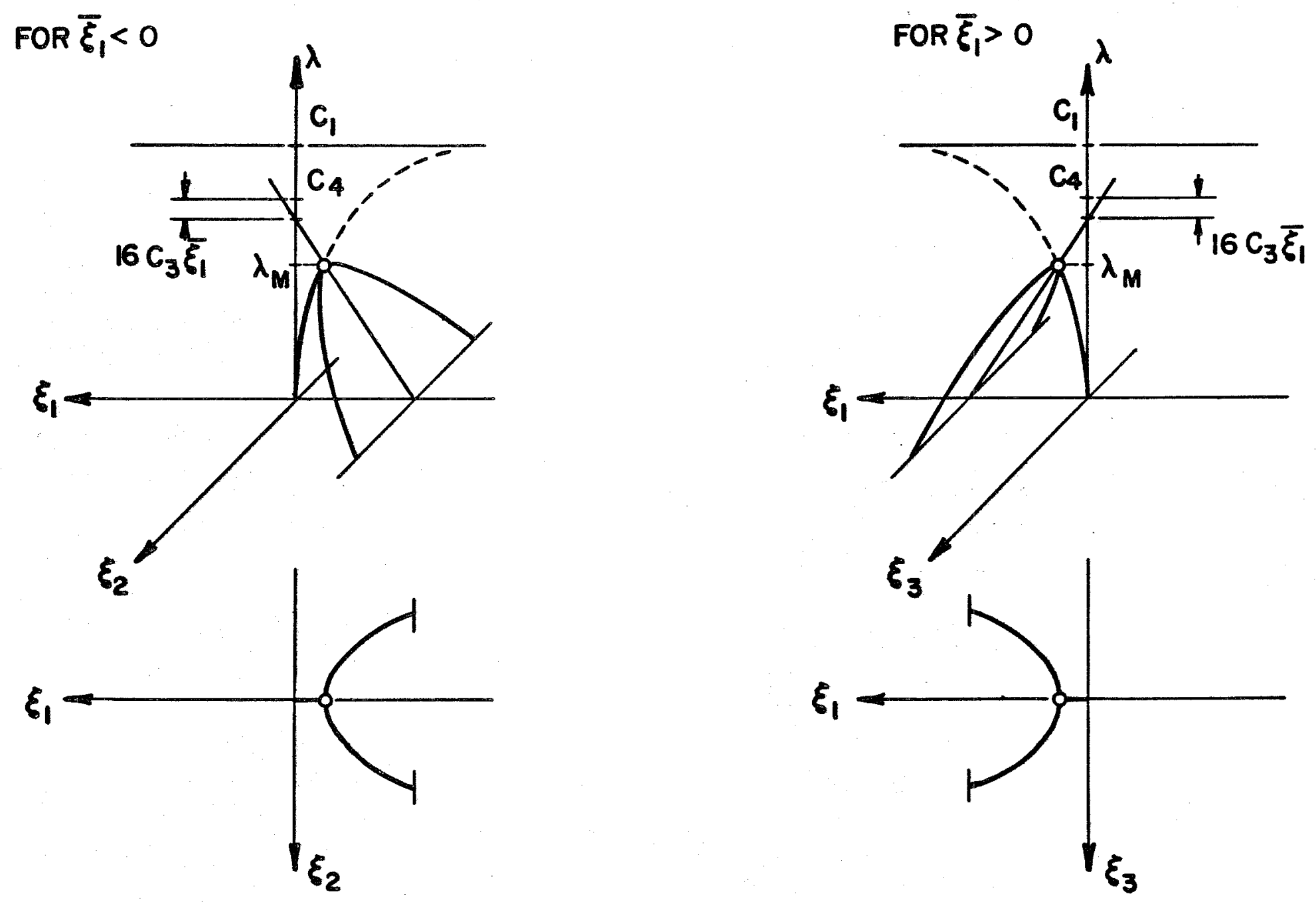

$\underline{\underline{v}}$

FIG. 47 POST BUCKLING EQUILIBRIUM PATHS FOR SHELLS WITH AXISYMMETRIC IMPERFECTIONS 
198

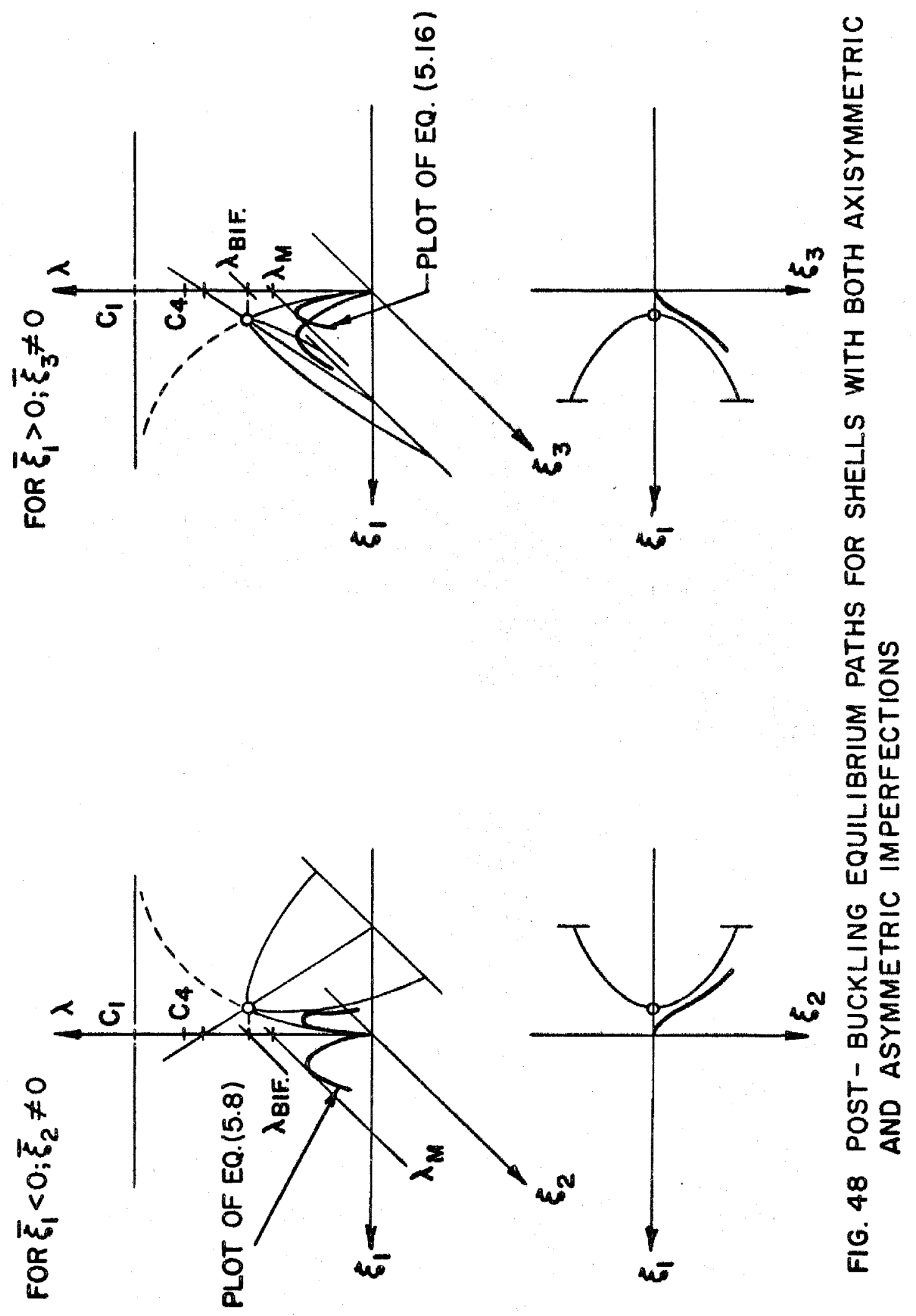

